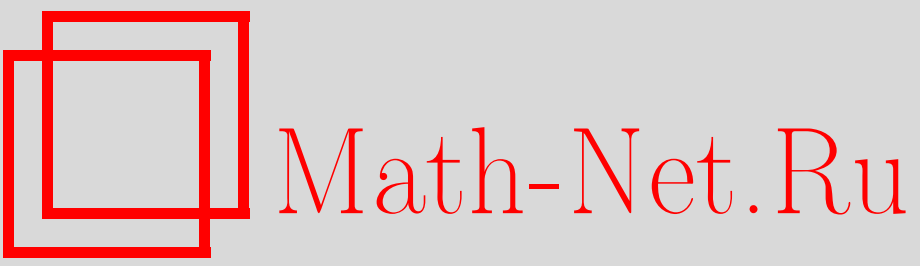

А. Н. Старков, Новый прогресс в теории однородных потоков, УМН, 1997, том 52, выпуск 4, 87-192

DOI: https://doi.org/10.4213/rm864

Использование Общероссийского математического портала Math-Net.Ru подразумевает, что вы прочитали и согласны с пользовательским соглашением

http://www.mathnet.ru/rus/agreement

Параметры загрузки:

IP : 54.205 .225 .156

26 апреля 2023 г., 16:41:30 


\title{
НОВЫЙ ПРОГРЕСС В ТЕОРИИ ОДНОРОДНЫХ ПОТОКОВ
}

\author{
A. Н. Старков
}

\section{СОДЕРЖАНИЕ}

$\S 0$. Соглашения, определения и необходимые сведения $\quad \ldots \ldots \ldots \ldots \ldots \ldots \ldots . .63$

$\S 1$. Поверхности постоянной отрицательной кривизны $\ldots \ldots \ldots \ldots \ldots \ldots \ldots . . \ldots 107$

$\S 2$. Метрическая жесткость орициклического потока ................ 110

$\S 3$. Геометрические обобщения теоремы Ратнер о метрической жест-

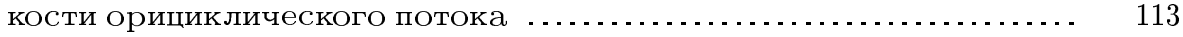

$\S 4$. Факторы и джойнинги орициклических потоков $\ldots \ldots \ldots \ldots \ldots \ldots \ldots \ldots . . \ldots \ldots$

$\S 5$. Жесткость, факторы и джойнинги унипотентных потоков . . ........ 119

$\S 6$. Динамика орициклического потока ............................. 123

$\S 7$. Классифокация эргодических мер унипотентных потоков . ......... 129

$\S 8$. Равномерная распределенность унипотентных траекторий $\ldots . . . . .136$

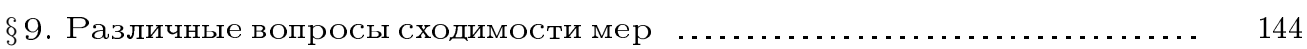

$\S 10$. Структура орбит, минимальных множеств и эргодических мер од-

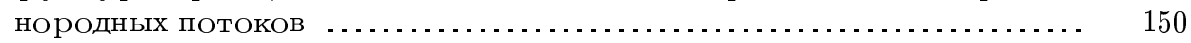

$\S 11$. Кратное перемешивание и метрическая жесткость однородных по-

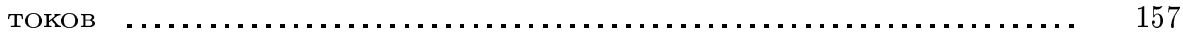

$\S 12$. Эргодические меры и замыкания орбит для действия произвольных

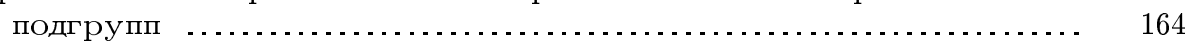

§ 13. Унипотентные потоки на однородных пространствах над локальны-

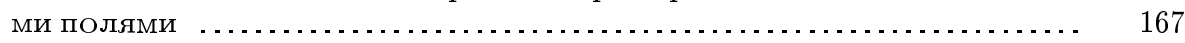

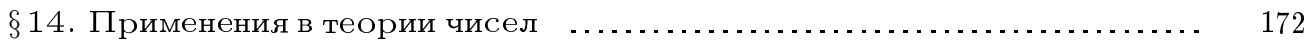

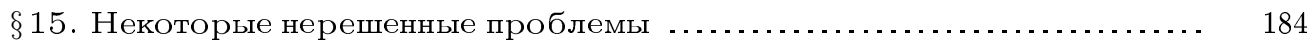

Список литературы . . . . . . . . . . . .

Один из наиболее естественных классов динамических систем возникает из следующей конструкции. Если $G$ - группа Ли и $F, \Gamma \subset G$ - две ее замкнутые подгруппы, то действие правыми сдвигами группы $F$ на левом однородном пространстве $\Gamma \backslash G$ называется однородным действием и обозначается $(\Gamma \backslash G, F)$ (в случае связной подгрупшы

Работа выполнена при финансовой поддержке Российского фонда фундаментальных исследований (грант № 95-01-00030) и Международного научного фонда (грант № M1E000). 
$F$ говорят об однородном потоке). По определению $\Gamma \backslash G$ имеет конечный объем, если $\Gamma \backslash G$ несет на себе конечную $G$-инвариантную меру $\nu$. Стандартным примером однородного действия является прямолинейный поток на $n$-мерном торе. Однако исторически интерес к этому классу динамических систем был вызван двумя другими его представителями: геодезическим и орициклическим потоками на единичном касательном расслоении $S M$ поверхности $M$ постоянной отрицательной кривизны. Здесь $S M=\Gamma \backslash G$, где $G=S L(2, \mathbb{R})$ и $\Gamma$ - ее дискретная подгруппа (содержашая центр $\left.\mathbb{Z}_{2} \subset G\right)$. Геодезический и орициклический потоки индуцированы соответственно подгруппами ${ }^{1}$

$$
A_{t}=\left(\begin{array}{cc}
e^{t / 2} & 0 \\
0 & e^{-t / 2}
\end{array}\right), \quad U_{s}=\left(\begin{array}{ll}
1 & s \\
0 & 1
\end{array}\right)
$$

Динамика этих двух потоков является весьма нетривиальной и обнаруживает сушественное различие между двумя типами однородных действий. Пожалуй, единственньм их сходством является то, что в предположении конечности $G$-инвариантного объема на $\Gamma \backslash G$ они эргодичны и перемешивают (Хопф [Но], Хедлунд [Не39]). Геодезический поток, будучи аносовским, обладает экспоненциальньм разбеганием траекторий и положительной энтропией (Синай [Si60]) и является представителем частично-гиперболических однородных потоков. Напротив, орициклический поток имеет полиномиальную скорость разбегания траекторий и нулевую энтропию (Гуревич $[\mathrm{Gu}]$, Дани [Da77]), что характерно для унипотентных однородных потоков.

Точнее, если все собственные значения присоединенных операторов $\operatorname{Ad}_{f}, f \in F$, на алгебре Ли g равны 1 (равны 1 по модулю), подгрупша $F$ назьвается унипотентной (соответственно квазиунипотентной). Благодаря Хедлунду [Не36] давно известно, что в предположении конечности объема $\Gamma \backslash G$ непериодические орбиты орициклического потока всюду плотны, причем в случае компактности $\Gamma \backslash G$ поток минимален (каждая его орбита плотна). Дани и Смилли [DS] доказали, что эргодические меры этого потока имеют аналогичную структуру: они либо сосредоточены на периодических траекториях, либо совпадают с $G$-инвариантной мерой объема на $\Gamma \backslash G$ (ранее Фюрстенберг [Fu72] установил, что в компактном случае орищиклический поток строго эргодичен, т.е. других эргодических мер на $\Gamma \backslash G$ нет; более современное доказательство, которое проходит и для равномерно параметризованного орициклического потока на поверхности переменной отрицательной кривизны, см. у Ратнер [R92]).

Известное соотношение $A_{-t} U_{s} A_{t}=U_{e^{-t} s}$ в точности означает, что орициклические орбиты образуют сжимающееся слоение для геодезического потока. Более обшим образом, пусть $G$ - полупростая группа Ли и сжимающееся слоение для частично-гиперболического потока $\left(\Gamma \backslash G, g_{\mathbb{R}}\right)$ образовано орбитами связной подгрупшы $U \subset G$ (такая подгруппа $U$ называется орисферической). Усиливая результаты Хедлунда и Фюрстенберга, Боуэн [Во76], Вич [V], Эллис и Перризо [ЕР] доказали минимальность и строгую эргодичность орисферического потока при условии его эргодичности и компактности $\Gamma \backslash G$. Впоследствии Дани [Da86a] при условии конечности объема $\Gamma \backslash G$ доказал, что замыкание любой орисферической орбиты на $\Gamma \backslash G$ является однородным подпространством. Если отбросить требование полупростоты групшы $G$,

\footnotetext{
${ }^{1}$ Любая одномерная подгруппа в $S L(2, \mathbb{R})$ сопряжена либо одной из указанных подгрупп, либо компактной подгруппе $S O(2, \mathbb{R})$, действие которой неинтересно с точки зрения динамики.
} 
но оставить условие компактности $\Gamma \backslash G$, то все орбиты орисферического потока устроены одинаково: их замькания изоморфны и совпадают с соответствуюшим эргодическим однородньм подпространством (Старков, [St91]).

С другой стороны, если группа Ли $G$ нильпотентна, то любой однородньй поток на $\Gamma \backslash G$ является унипотентным, и в случае эргодичности относительно конечной $G$-инвариантной меры на $\Gamma \backslash G$ поток является минимальным (Ауслендер, $[\mathrm{AGH}])$ и строго эргодичным (Фюрстенберг [Fu61]). Для неэргодического потока замькание любой орбиты является однородным подпространством [P69] (см. также [St84], [Le]). Heтрудно также доказать, что замыкание унипотентной траектории на разрешимом однородном пространстве $\Gamma \backslash G$ является однородным подпространством в $\Gamma \backslash G$ (для конечного объема это доказано в [St84], общий случай рассмотрен в [St87b] и [R90b]).

Эти результаты наводят на мысль о том, что замыкания орбит и эргодические меры унипотентного потока на произвольном однородном пространстве $\Gamma \backslash G$ конечного объема должны иметь алгебраическое происхождение. Так, замыкание орбиты $\overline{\Gamma U} \subset \Gamma \backslash G$ должно совпадать с орбитой $\Gamma H$ некоторой подгруппы $H \subset G$, причем на $\Gamma H$ должна сушествовать конечная $H$-инвариантная мера $\mu$ (в таком случае иногда мы будем говорить, что замыкание орбиты $\overline{\Gamma U}$ и мера $\mu$ являются алгебраическими). Интерес к подобного рода утверждениям резко возрос, когда в середине 70-х годов Рагунатан обнаружил замечательную связь между унипотентными потоками и решением диофантовых неравенств для квадратичных форм. А именно, если установить, что все компактные замькания орбит потока $\left(S L(3, \mathbb{Z}) \backslash S L(3, \mathbb{R}), u_{\mathbb{R}}\right)$, где $u_{t}=\left(\begin{array}{ccc}1 & t & t^{2} / 2 \\ 0 & 1 & t \\ 0 & 0 & 1\end{array}\right)$, являются орбитами других подгрупш в $S L(3, \mathbb{R})$, то была бы доказана старая гипотеза Опенгейма-Давенпорта из теории чисел: неопределенная невырожденная форма $B\left(x_{1}, \ldots, x_{n}\right)$ от $n \geqslant 3$ переменных в целых точках принимает значения, сколь угодно близкие к 0 при условии, что $B$ не пропорциональна форме с рациональными коэффициентами. Основная трудность при этом заключена в том, что $u_{\mathbb{R}}$ не является орисферической подгруппой в $G$ (хотя и содержится в таковой).

После того, как обнаружилась связь с теорией чисел, проблема изучения динамики унипотентных потоков стала центральной для данной теории в 80-90-х гг. В явном виде гипотезы об алгебраичности замыканий орбит и эргодических мер были сформулированы в работе Дани [Da81] (на самом деле Дани высказал их для однопараметрических подгрупп $U \subset G$ и редуктивных груп $G$; обшая их формулировка - для произвольной группы Ли $G$ и произвольной подгруппы $U \subset G$, порожденной унипотентными элементами, дана Маргулисом в [Ma88], [Ma91b]). Первая из них получила название топологической гипотезы Рагунатана, вторую же мы будем назьвать метрической гипотезой Дани (иногда она назьвается метрической гипотезой Рагунатана).

С некоторой степенью условности развитие теории однородных потоков можно разбить на три этапа. Первый из них в основном был посвящен классическому геометрическому подходу Морса, Хопфа и Хедлунда к изучению динамики геодезического и орициклического потоков в 20-40-х гг. (как известно, в дальнейшем эти работы привели к созданию теории гладких динамических систем с гиперболическим поведением в работах Смейла, Аносова, Синая, Боуэна, Песина и др.). Началом второго можно, пожалуй, считать работу Гельфанда и Фомина $[\mathrm{GF}]$, в которой они впервые заметили, 
что эти потоки вписьваются в более обшую конструкцию однородных потоков и это позволяет для их изучения применять теорию представлений групп. Этот подход был успешно развит американской школой в начале 60-х гг., когда появилось первое систематическое изложение данной теории в трудах конференции по Анализу в целом, проводившейся в 1960/61 гг. в Йельском университете [AGH]. На этом этапе основной задачей было нахождение критерия эргодичности и вычисление энтропии и спектра однородных потоков последовательно для классов нильпотентных, разрешимых, полупростых и общих групп Ли. Эти вопросы для отдельных классов однородных потоков были рассмотрены Ауслендером и Грином [AGH], [AG], [Au], Муром [Mo66], Степиным [Ste69], [Ste73], Боуэном [Во71] и другими. Развивая эти результаты, Дани [Da77], а также Брезин и Myp [BM] установили критерий эргодичности и вычислили энтропию и спектр однородных потоков в общем случае. Позднее в работах автоpa [St83], [St89] была проведена редукщия неэргодической ситуашии к эргодическому случаю. Более подробное изложение всех упомянутых результатов можно найти в обзоре $[\mathrm{SSS}]$.

Как уже было сказано, третий этап теории однородных потоков был в основном посвящен доказательству гипотез Рагунатана и Дани для унипотентных потоков и их приложениям после того, как они были сформулированы в [Da81]. Основные события здесь происходили следующим образом. В конце 80-х годов Маргулис опубликовал доказательство гипотезы Оппенгейма-Давенпорта в [Mа87], [Mа89], разобрав ситуацию с топологической гипотезой Рагунатана для $G=S L(3, \mathbb{R})$ (позднее этот результат был усилен в цикле совместных работ Дани и Маргулиса [DM89,90a,90b]). B ceрии работ [R90b,90c,91a] Марина Ратнер доказала метрическую гипотезу, а в работе $[\mathrm{R} 91 \mathrm{~b}]$ - и топологическую (одновременно Шах [Sh91] доказал ее для регулярных унипотентных подгрупп в полупростых группах $\mathbb{R}$-ранга 1). При этом обнаружилось, что центральной является именно метрическая теорема, из которой уже вьводится и топологическая. Новьй (и возможно более эффективный) способ такого вывода был предложен Дани и Маргулисом [DM93]. Проблемы теории чисел привели к необходимости доказательства гипотез Рагунатана и Дани для груп Ли над локальными полями. В такой постановке обе гипотезы были доказаны Ратнер [R95a]; независимо от нее и другим способом (используя идеи работ [R90b,90c], [BP], [DM89-93], [Ma87,89,91b]) Маргулис и Томанов [МТ94,96] доказали метрическую гипотезу. Одновременно были найдены и новые приложения этих результатов в теории чисел, а также в самой теории однородных потоков.

Данньй обзор посвящен описанию результатов, полученных на третьем этапе теории однородных потоков за последние 10-15 лет. Следует еше раз отметить, что именно в это время интерес к данной области повысился ввиду найденных приложений в теории чисел. Достаточно напомнить, что на последних двух Международных Конгрессах Математиков теории однородных потоков были посвящены три доклада, два из которых (Маргулиса в Киото, 1990 г., и Ратнер в Цюрихе, 1994 г.) были пленарными. Многие (но не все) вопросы, затронутые в данной работе, рассматривались также в обзорах [R84,94,95b], [Ma91b,96], [Gh], [B], [Da95].

Несколько слов о структуре нашего обзора. Пожалуй, первым сушественным ингредиентом для доказательства гипотез Рагунатана и Дани, полученным еще до их формулировки в [Da81], является весьма нетривиальный результат Маргулиса [Ma71] 
о том, что унипотентная траектория в $S L(n, \mathbb{Z}) \backslash S L(n, \mathbb{R})$ не может уходить на бесконечность (напомним, что это утверждение было использовано Маргулисом для доказательства его знаменитой теоремы арифметичности). Позднее этот результат был усилен Дани $[\mathrm{Da} 84,86 \mathrm{~b}]$, который в итоге доказал возврашаемость с положительной плотностью времен произвольной унипотентной траектории в некоторый компакт. Мы приводим формулировки этих теорем в списке предварительных сведений в $\S 0$ и демонстрируем их доказательство на простейшем примере.

C другой стороны, в 80-х гг. Мариной Ратнер [R82] были получены замечательные (и весьма неожиданные даже для специалистов) результаты о жесткости орициклического потока: метрический изоморфизм двух орициклических потоков почти всюду совпадает с аффинным отображением (т.е. имеет алгебраическое происхождение). Основным инструментом при этом было свойство, известное теперь как $R$-свойство орищиклического потока, которое описывает характер разбегания его близких траекторий (в дальнейшем именно это свойство интенсивно применялось Ратнер для доказательства гипотез Рагунатана и Дани). На этих результатах мы остановимся намного подробнее (в частности, потому что они были недостаточно освещены в обзоpe [SSS]). Предварительно в $\S 1$ мы приводим краткие сведения из гиперболической геометрии, а в $\S 2$ рассматриваем динамику геодезического и орищиклического потоков и формулируем теорему о метрической жесткости орициклического потока. В 33 обсуждаются геометрические обобщения данной теоремы, полученные Фельдманом и Орнстейном [FO], Фламинио и Спатциером $[\mathrm{F}],[\mathrm{FS}]$ при изучении орисферических слоений на многообразиях отрицательной кривизны.

Теоремы об алгебраичности факторов и джойнингов орищиклического потока, доказанные Ратнер в [R82a,82b], обсуждаются в $\S 4$. В $\S 5$ описано, как эти результаты и свойство метрической жесткости обобщаются на класс произвольных унипотентных потоков (здесь основной вклад сделан Витте, который, в частности, доказал в [Wi85] аффинность изоморфизма унипотентных эргодических потоков, а в [Wi94] изучил строение их факторпотоков. Заметим, что для нильпотоков эти результаты были получены еще в 70-х годах в работе Перри [P71]). Также мы показываем, что эти результаты легко выводятся из метрической гипотезы Дани (и поэтому они естественно вписьваются в круг рассматриваемых вопросов).

Само доказательство метрической гипотезы слишком сложно, чтобы воспроизводить его в рамках обзора. Чтобы дать представление о нем, мы пользуемся способом, который избрала в [R92] сама Марина Ратнер: в §6 мы даем это доказательство для орициклического потока (не пользуясь тем фактом, что сжимающееся слоение для геодезического потока образовано орбитами орищиклического потока), а в $\S 7$ - показываем, как это доказательство служит основой для рассмотрения произвольных унипотентных потоков. Параллельно мы намечаем схему доказательства метрической гипотезы из [МТ94].

Что касается вывода топологической гипотезы Рагунатана из метрической, то мы избрали путь, предложенный Дани и Маргулисом в [DM93]. В отличие от самой работы [DM93] мы для иллюстрации вначале даем доказательство для орициклического потока, а затем уже подробно рассматриваем обший случай. Для однопараметрических потоков топологическая теорема может быть значительно усилена: унипотентная траектория не только имеет однородное замыкание, но и равномерно распределена 
в нем. Впервые это предположение было высказано в [Da82] (для $S L(n, \mathbb{R}))$ и [Ma88] (в общем случае), а доказано в [R91b] и [DM93]. Фактически здесь речь идет о сходимости в $*$-слабой топологии некоторой последовательности мер к алгебраической мере в пространстве вероятностных мер $\mathscr{P}(\Gamma \backslash G)$. Обобщения этой теоремы, принадлежашие Дани и Маргулису [DM93], Ратнер [R94], Эскину, Мозесу и Шаху [MS], [Sh94], [EMS96,97], приводятся в $\S$ 9. Так, показано, что семейство удлиняюшихся кусков траекторий имеет свойство равномерной распределенности при условии, что среди предельных точек есть хотя бы одна в общем положении (не лежащая в инвариантном подпространстве меньшей размерности). При этом могут варьироваться не только траектории, но и сами унипотентные подгрупшы [DM93]. Пространство вероятностных эргодических мер для унипотентных подгрупп оказывается замкнутым [MS]; аналогичные результаты об алгебраичности предельной меры для семейства сдвигов эргодической алгебраической меры получены в [EMS96,97]. Само свойство равномерной распределенности (и алгебраичности замыкания) имеет силу не только для унипотентных траекторий, но и для полиномиальных кривых в $S L(n, \mathbb{R})[\operatorname{Sh} 94]$.

В $\S 10$ и $§ 11$ показывается, как результаты Ратнер могут быть применены для изучения произвольных однопараметрических потоков. Здесь имеются два подхода: 1) показать, что какие-либо свойства не очень сильно ухудшаются, если рассмотреть более общий класс квазиунипотентных потоков, и заведомо нарушаются для противоположного класса частично-гиперболических потоков; 2) постараться общую задачу свести к унипотентному случаю. Примером первого подхода являются критерий для гладкости замьканий всех орбит потока и свойство послойной аффинности для изоморфизма двух эргодических квазиунипотентных потоков, второго - классификация минимальных множеств и доказательство гипотезы Маркуса о кратном перемешивании. Эти результаты принадлежат автору ([St90,93,95], [St95a]). В вопросе существования минимальных множеств мы используем недавний результат Клейнбока и Маргулиса [KM96] о хаусдорфовой размерности семейства ограниченных орбит.

В $\S 12$ рассматривается вопрос об устройстве эргодических мер и замыканий орбит для действия многомерных связных подгрупп $F \subset G$. Здесь множество вопросов еше остается открытыми. Тем не менее, для некоторых подгруп $F \subset G$, содержащих унипотентные элементы, но не порожденных ими, как эргодические меры, так и замыкания орбит имеют алгебраическое происхождение: в этой связи приводятся результаты Ратнер [R94], Мозеса [Moz95a], Шаха [Sh96], Маргулиса и Томанова [MT96]. В случае, когда $F$ вообще не содержит (квази)унипотентных элементов, частичные результаты недавно получены Катком и Спатциером [KS96].

Приложениям в теории чисел посвящены $\S 13$ и $\S 14$. Вначале приводятся краткие сведения из теории груп Ли над $p$-адическим полем и весьма полезная информация об устройстве однопараметрических подгрупп и структуре пространств конечного объема, полученная Ратнер в [R93,R95a]. В конще $\S 13$ приводится сводка результатов о динамике унипотентных потоков на однородных пространствах произведений нескольких групп Ли над локальными полями нулевой характеристики (см. также [MT94,96]).

В $\S 14$, следуя [DM90b] и [Ma90b], полностью доказьвается гипотеза Оппенгейма-Давенпорта с помошью изучения частного случая $(G=S L(3, \mathbb{R}))$ топологической гипотезы Рагунатана. Затем приводятся различные обобщения, относящиеся к за- 
даче о плотности значений иррациональной невырожденной неопределенной квадратичной формы $Q$ (над произведением локальных полей) в примитивных узлах целочисленной решетки и принадлежашие Дани и Маргулису [DM89,90a,93], а также Борелю и Прасаду [BP], [В]. Вопрос об асимптотике числа целых решений неравенства $0<|Q(x)|<\varepsilon$ в шаре $|x|<R$ при $R \rightarrow \infty$ для неопределенных форм $Q$ был недавно решен в работах Дани и Маргулиса [DM93] (оценка снизу), а также Эскина, Маргулиса и Мозеса [EMM] (оценка сверху). Изложение здесь довольно сжатое, так как этот предмет хорошо освещен в обзорах [Ма96] и (кроме последней работы) [В]. Более подробно рассматривается работа Эскина, Мозеса и Шаха [EMS96] об асимптотике числа целых точек на $\mathbb{Q}$-алгебраических многообразиях в $\mathbb{R}^{n}$. Формулируются также недавнее доказательство Клейнбоком и Маргулисом [KM97] гипотез Бейкера и Спринджука из теории чисел и результаты Скриганова [Skr] об асимптотике числа целых точек внутри многогранника.

Наконец, в $§ 15$ мы вкратце упоминаем о нерешенных проблемах в теории однородных потоков, примыкаюших к данной тематике.

Несколько слов о системе обозначений, принятых в данной работе. Здесь мы будем рассматривать левые однородные пространства с правым действием сдвигами на них. Такой точки зрения во всех своих работах придерживаются Ратнер, Витте и др., хотя более общепринят противоположный подход. Наш выбор в пользу “левой" системы обозначений объясняется не только тем, что обзор работ Ратнер занимает здесь весьма значительньй объем, но и тем, что алгебраический подход при изучении геодезического и орициклического потоков приводит именно к левым однородным пространствам.

В заключение автор выражает признательность Д. В. Аносову, С. Дани, Г. А. Маргулису, М. Ратнер, А. М. Степину и Н. Шаху за полезные консультации.

\section{$\S 0$. Соглашения, определения и необходимые сведения}

А. Эргодическая теория и топологическая динамика. Мерой $\mu$ на множестве $X$ мы будем называть неотрицательную счетно-аддитивную функцию $\mu: \Omega(X) \rightarrow$ $\mathbb{R}^{+} \cup \infty$ на некоторой $\sigma$-алгебре $\Omega(X)$ подмножеств из $X$. При этом подмножества из $\Omega(X)$ назьваются измеримыми множествами. Если $\mu(X)<\infty$, то мера $\mu$ назьвается конечной, в противном случае - бесконечной. Конечную меру $\mu$, нормированную условием $\mu(X)=1$, будем называть вероятностной. Пару $(X, \mu)$ (опуская для краткости $\Omega(X))$ назовем пространством с мерой. Две конечные меры $\mu$ и $\mu^{\prime}$ на $X$ называются эквивалентными $\left(\mu \sim \mu^{\prime}\right)$, если $\mu$ и $\mu^{\prime}$ имеют одну и ту же $\sigma$-алгебру измеримых подмножеств и $\mu(A)=0 \Longleftrightarrow \mu^{\prime}(A)=0$ для всякого измеримого $A \subset X$. Меры $\mu$ и $\mu^{\prime}$ взаимно сингулярны, если найдется такое $A \subset X$, что $\mu(A)=0$ и $\mu^{\prime}(X-A)=0$.

Если имеется отображение $f: X \rightarrow X^{\prime}$ и пространство $X$ снабжено мерой $\mu$, то на $X^{\prime}$ можно задать меру $\mu^{\prime}=f_{*} \mu$ по правилу $\mu^{\prime}(A)=\mu\left(f^{-1}(A)\right)$ для каждого $A \subset X^{\prime}$ с измеримым прообразом $f^{-1}(A)$.

Пусть $(X, \mu)$ и $\left(X^{\prime}, \mu^{\prime}\right)$ - два пространства с мерами. Отображение $f: X \rightarrow X^{\prime}$ называется измеримым, если прообраз $f^{-1}(A)$ каждого измеримого множества $A \subset X^{\prime}$ измерим. Измеримое отображение $f: X \rightarrow X^{\prime}$ назьвается сохраняюшим меру, если $f_{*} \mu=\mu^{\prime}$, т.е. $\mu\left(f^{-1}(A)\right)=\mu^{\prime}(A)$ для каждого измеримого $A \subset X^{\prime}$. 
Автоморфизмом измеримого пространства $(X, \mu)$ назовем сохраняющее меру обратимое mod 0 отображение $f: X \rightarrow X$. Через $\operatorname{Aut}(X, \mu)$ обозначим группу всех автоморфизмов пространства $(X, \mu)$.

Пусть $F$ - топологическая група. Всюду далее будем предполагать, что $F$ является локально-компактной и сепарабельной. ${ }^{2}$ Непрерьвным действием $F$ на пространстве $(X, \mu)$ (мы будем рассматривать только такие действия) называется гомоморфизм $\varphi: F \rightarrow \operatorname{Aut}(X, \mu)$ такой, что для каждых измеримых $A, B \subset X$ функция $\mu\left(\varphi_{g}(A) \cap B\right)$ непрерывна по $g \in F$. В случае $F=\mathbb{R}$ непрерьвное действие назьвают потоком, а в случае $F=\mathbb{Z}$ - каскадом. Измеримое множество $A \subset X$ называют $F$-инвариантным, если $\varphi_{g}(A)=A$ для всех $g \in F$. Действие $F$ на $(X, \mu)$ является эргодическим, если для каждого $F$-инвариантного множества $A \subset X$ либо $\mu(A)=0$, либо $\mu(X-A)=0$ (одновременно $\mu$ называется $F$-эргодической мерой $)$.

Для $F=\mathbb{R}$ непрерьвньй поток на $(X, \mu)$ будем обозначать $\left(X, \varphi_{\mathbb{R}}\right)$. Предположим, что $\mu$ - эргодическая мера для потока $\left(X, \varphi_{\mathbb{R}}\right)$. Тогда имеет место эргодическая теорема Биркгофа-Хинчина: для каждой $f \in L^{1}(X, \mu)$ и для $\mu$-почти каждой точки $x \in X$ имеем

$$
\frac{1}{T} \int_{0}^{T} f\left(\varphi_{t} x\right) d t \rightarrow f^{*}=\frac{\int_{X} f d \mu}{\mu(X)}, \quad T \rightarrow \infty
$$

В частности, если $\mu(X)=\infty$, то $f^{*}=0$.

Предположим теперь, что мера $\mu$ является вероятностной. Тогда эргодичность потока $\left(X, \varphi_{\mathbb{R}}\right)$ эквивалентна тому, что кроме констант нет других $\varphi_{\mathbb{R}}$-инвариантных функций из $L^{1}(X, \mu)$. Из теоремы Биркгофа-Хинчина вытекает, что различные $\varphi_{\mathbb{R}}$-эргодические вероятностные меры взаимно сингулярны.

Поток на пространстве $(X, \mu)$ с вероятностной мерой назьвается перемешиваюшим, если для любых измеримых $A, B \subset X$

$$
\lim _{t \rightarrow \infty} \mu\left(\varphi_{t} A \cap B\right)=\mu(A) \mu(B) .
$$

Более общее понятие: поток назьвается $k$-перемешиваюшим, если для любых $k+1$ измеримых множеств $A_{0}, \ldots, A_{k} \subset X$ и любых $k+1$ последовательностей $\left\{t_{i}(0)\right\}, \ldots,\left\{t_{i}(k)\right\}$ таких, что $\left|t_{i}(l)-t_{i}(m)\right| \rightarrow \infty, i \rightarrow \infty, l \neq m$, вьполняется равенство

$$
\lim _{i \rightarrow \infty} \mu\left(\bigcap_{l=0}^{k} \varphi_{t_{i}(l)} A_{i}\right)=\prod_{l=0}^{k} \mu\left(A_{i}\right) .
$$

Ясно, что перемешиваюший поток эргодичен. Примером эргодического неперемешиваюшего потока является прямолинейньй поток на торе $\mathbb{Z}^{2} \backslash \mathbb{R}^{2}$, действующий по правилу $\varphi_{t}\left(\mathbb{Z}^{2}+a\right)=\left(\mathbb{Z}^{2}+a+t v\right)$ сдвигами вдоль вектора $v \in \mathbb{R}^{2}$ с рационально независимыми координатами. В общем случае не известно (проблема Рохлина), следует ли из обычного перемешивания перемешивание всех степеней?

В эргодической теории имеется также важное понятие метрической энтропии $0 \leqslant e(\varphi) \leqslant \infty$, измеряющей "степень хаотичности" траекторий автоморфизма

\footnotetext{
${ }^{2}$ На самом деле все наши группы будут группами Ли.
} 
$\varphi \in \operatorname{Aut}(X, \mu)$. Точное определение энтропии можно найти в $[\mathrm{KSF}]$. Нам будет важен лишь критерий нетривиальности энтропии С.3 для однородных потоков, поэтому мы не воспроизводим полного определения. Энтропии сдвигов потока связаны между собой формулой $e\left(\varphi_{t}\right)=|t| e\left(\varphi_{1}\right)$. Для каждого потока согласно Пинскеру [Pi] существует единственньй (с точностью до изоморфизма) максимальный факторпоток нулевой энтропии. Если такой факторпоток тривиален (т.е. все нетривиальные факторы исходного потока имеют положительную энтропию), то исходный поток называется $K$-потоком или потоком с $K$-свойством. ${ }^{3}$ Известно, что $K$-поток обладает перемешиванием всех степеней.

Предположим, что $\left(X, \varphi_{\mathbb{R}}\right)$ и $\left(X^{\prime}, \varphi_{\mathbb{R}}^{\prime}\right)$ - два потока на пространствах с мерами. Измеримое отображение $\alpha: X \rightarrow X^{\prime}$ назьвается сопряженностью потоков (или эквивариантным относительно потоков), если

$$
\alpha\left(\varphi_{t} x\right)=\varphi_{t}^{\prime} \alpha(x) \text { для } \mu \text {-почти всех } x \in X, \forall t \in \mathbb{R} \text {. }
$$

Если сопряженность $\alpha$ сохраняет меру, она называется метрической сопряженностью. При этом поток $\left(X, \varphi_{\mathbb{R}}\right)$ называется накрываюшим, а поток $\left(X^{\prime}, \varphi_{\mathbb{R}}^{\prime}\right)$ - фактором. Если к тому же $\alpha$ является обратимьм, то потоки называются метрически изоморфными. Эргодичность, $k$-перемешивание, $K$-свойство, энтропия являются метрическими инвариантами, т.е. сохраняются при метрическом изоморфизме.

Потоки $\left(X, \mu, \varphi_{\mathbb{R}}\right)$ и $\left(X^{\prime}, \mu^{\prime}, \varphi_{\mathbb{R}}^{\prime}\right)$ называются эквивалентными по Какутани, если найдется отображение $\alpha: X \rightarrow X^{\prime}$ такое, что $\alpha_{*} \mu \sim \mu^{\prime}$ и $\alpha$ переводит (почти все) $\varphi_{\mathbb{R}}$-орбиты в $\varphi_{\mathbb{R}}^{\prime}$-орбиты с сохранением направления (т.е. положительные полуорбиты в положительные, а отрицательные - в отрицательные). Эквивалентность по Какутани сохраняет свойство эргодичности, но не сохраняет свойство перемешивания. Энтропия, вообще говоря, также не сохраняется, однако свойства обращения в нуль и конечности энтропии при эквивалентности по Какутани остаются неизменными.

Пусть $\left(X, \mu, \varphi_{\mathbb{R}}\right)$ и $\left(X^{\prime}, \mu^{\prime}, \varphi_{\mathbb{R}}^{\prime}\right)$ - два потока, сохраняющих меру. Через $p: X \times X^{\prime} \rightarrow X$ и $p^{\prime}: X \times X^{\prime} \rightarrow X^{\prime}$ обозначим проекции. Джойнингом этих потоков назьвается такая инвариантная относительно диагонального потока $\varphi_{t} \times \varphi_{t}^{\prime}$ мера $m$ на $X \times X^{\prime}$, что $p$ и $p^{\prime}$ сохраняют меры (т.е. $m\left(A \times X^{\prime}\right)=\mu(A)$ для каждого измеримого $A \subset X$ и, аналогично, $m\left(X \times A^{\prime}\right)=\mu^{\prime}\left(A^{\prime}\right)$ для каждого измеримого $\left.A^{\prime} \subset X^{\prime}\right)$. Если кроме $\mu \times \mu^{\prime}$ нет других джойнингов для $\varphi_{\mathbb{R}}$ и $\varphi_{\mathbb{R}}^{\prime}$, то меры $\mu$ и $\mu^{\prime}$ назьваются дизъюнктными. В случае $\left(X, \mu, \varphi_{\mathbb{R}}\right)=\left(X^{\prime}, \mu^{\prime}, \varphi_{\mathbb{R}}^{\prime}\right)$ джойнинг $m$ на $X \times X$ называется автоджойнингом (от "self-joining") потока. Кроме $\mu \times \mu$ на $X \times X$ есть другие примеры автоджойнингов: диагональная мера $\hat{\mu}(A \times B)=\mu(A \cap B)$ и ее сдвиги $\hat{\mu}_{t}(A \times B)=\mu\left(\varphi_{t} A \cap B\right)$. Эти автоджойнинги называются тривиальными. Аналогично вводятся автоджойнинги произвольной степени: мера $m$ на $X^{n}$ является автоджойнингом $n$-й степени, если она инвариантна относительно диагонального потока $\varphi_{t}^{n}$ и ее проекции на все копии $X$ совпадают с $\mu$. Подробное изложение теории джойнингов можно найти в обзоре Тувено [Th].

\footnotetext{
${ }^{3}$ На самом деле обычно испольуется эквивалентное данному первоначалное определение Колмогорова [KSF].
} 
Пусть $X$ - топологическое пространство, которое всюду далее будем предполагать локально-компактным и сепарабельным. ${ }^{4}$ Действием топологической группы $F$ на $X$ назьвается непрерьвньй гомоморфизм $\alpha: F \rightarrow \operatorname{Homeo}(X)$ в группу всех гомеоморфизмов пространства $X$, снабженную топологией равномерной сходимости на компактах. Действие называется топологически транзитивным, если найдется точка $x \in X$ с плотной $F$-орбитой ${ }^{5}$, и минимальньм, если каждая точка имеет плотную $F$-орбиту. $F$-инвариантное замкнутое подмножество $A \subset X$ назьвается минимальным, если каждая $F$-орбита внутри $A$ является плотной в $A$. По лемме Цорна каждьй поток на компактном пространстве обладает минимальным множеством.

Рассмотрим поток $\left(X, \varphi_{\mathbb{R}}\right)$ на топологическом пространстве $X$. Каждая точка $x \in X$ имеет две полуорбиты: положительную $\varphi_{\mathbb{R}^{+}} x$ и отрицательную $\varphi_{\mathbb{R}^{-}} x$. Положительная полуорбита точки $x \in X$ называется рекуррентной 6 , если она возврашается в сколь угодно малую окрестность точки $x$, т.е. найдется последовательность $t_{k} \rightarrow+\infty$ такая, что $\varphi_{t_{k}} x \rightarrow x$ (аналогично определяется рекуррентность в отрищательном направлении).

Нерекуррентная полуорбита является локально-замкнутой. Положительная полуорбита называется дивергентной или уходящей на бесконечность, если для любого компакта $K \subset X$ найдется число $T>0$ такое, что $\varphi_{t} x \notin K$ для всех $t \geqslant T$.

Точка $x \in X$ называется блуждающей, если найдутся окрестность $O(x) \subset X$ и число $T>0$ такие, что $\varphi_{t}(O(x)) \cap O(x)=\varnothing$ для всех $t \geqslant T$. Ясно, что обе полуорбиты блуждающей точки нерекуррентны. Множество $\Omega \subset X$ всех неблуждающих точек является замкнутым и инвариантным.

Поток $\left(X, \varphi_{t}\right)$ называется топологически перемешиваюшим, если для любых двух открытых множеств $U, V \subset X$ найдется $T>0$ такое, что $\varphi_{t}(U) \cap V \neq \varnothing$ для всех $t \geqslant T$. Поток на метрическом пространстве $(X, d)$ назьвается равномерно непрерывным, если для каждого $\varepsilon>0$ найдется $\delta>0$ такое, что из $d(x, y)<\delta$ вытекает $d\left(\varphi_{t} x, \varphi_{t} y\right)<\varepsilon$ для всех $t \in \mathbb{R}$. Замыкание любой орбиты равномерно непрерьвного потока является минимальньм множеством. Ясно, что равномерно непрерьвньй поток не может быть топологически перемешивающим.

Предположим, что $\mu$ - борелевская мера на топологическом пространстве $X$ (это означает, что $\sigma$-алгебра $\Omega(X)$ измеримых множеств получена пополнением $\sigma$-алгебры борелевских множеств в $X$ и для любого множества $A \subset X$ конечной меры и любого $\varepsilon>0$ найдутся компакт $K \subset A$ и открытое множество $U \supset A$ такие, что $\mu(U-K)<\varepsilon)$. В дальнейшем борелевские меры предполагаются локально-конечными, т.е. конечными на компактных подмножествах в $X$.

Если борелевская мера $\mu$ конечна и инвариантна относительно однопараметрического потока, то по теореме Пуанкаре о возврашении $\mu$-почти все траектории рекуррентны в обоих направлениях.

\footnotetext{
${ }^{4} \mathrm{~B}$ действительности мы будем рассматривать замкнутые подмножества в однородных пространствах групп Ли.

${ }^{5}$ Для $F=\mathbb{Z}$ или $\mathbb{R}$ обычно исключают вырожденный случай, когда плотная траектория локально замкнута и некомпактна (это может произойти для $\operatorname{dim}(X)=\operatorname{dim}(F)$ ).

${ }^{6}$ Мы ползуемся терминологией Готтшалка-Хедлунда. По Биркгофу свойство рекуррентности гораздо более ограничительно и в компактном случае совпадает со свойством минимальности замыкания.
} 
Носителем $\operatorname{supp}(\mu)$ борелевской меры $\mu$ (в топологическом смысле) назьвается наименьшее замкнутое множество полной меры. Предположим, что $\operatorname{supp}(\mu)=X$, т.е. мера каждого открытого множества положительна (такая мера называется строго положительной). Тогда из эргодичности потока $\left(X, \varphi_{\mathbb{R}}\right)$ вытекает его топологическая транзитивность (более того, почти все точки из $X$ имеют плотные орбиты в $X$ ). Если мера $\mu$ конечна, то из метрического перемешивания следует топологическое. Мера $\mu$ на римановом многообразии называется гладкой, если она эквивалентна мере объема (ясно, что гладкая мера строго положительна).

Поток $\left(X, \varphi_{t}\right)$ называется строго эргодическим, если вероятностная инвариантная борелевская мера единственна. ${ }^{7}$ Примером такого потока является прямолинейный эргодический поток на торе.

Для топологического пространства $X$ через $\mathscr{P}(X)$ обозначим афффинное пространство вероятностных мер на $X$ с $*$-слабой топологией, т.е. $\mu_{n} \rightarrow \mu$, если $\mu_{n}(f) \rightarrow \mu(f)$ для каждой непрерьвной функции $f$ на $X$ с компактньм носителем (пространство таких функщий дальше будем обозначать через $C_{c}(X)$ ). Известно, что для компактного $X$ пространство $\mathscr{P}(X)$ также компактно. Отсюда легко вьводится, что любой каскад или поток на компактном пространстве всегда обладает инвариантной вероятностной мерой. В частности, строго эргодический каскад (поток) является минимальным, если единственная инвариантная вероятностная мера строго положительна.

Потоки $\left(X, \varphi_{\mathbb{R}}\right)$ и $\left(X^{\prime}, \varphi_{\mathbb{R}}^{\prime}\right)$ назьваются топологически изоморфными, если найдется гомеоморфизм $\alpha: X \rightarrow X^{\prime}$, устанавливающий их сопряженность:

$$
\alpha\left(\varphi_{t} x\right)=\varphi_{t}^{\prime} \alpha(x), \forall x \in X, \forall t \in \mathbb{R}
$$

Если гомеоморфизм $\alpha$ переводит $\varphi_{\mathbb{R}}$-орбиты в $\varphi_{\mathbb{R}}^{\prime}$-орбиты с сохранением направления, то потоки назьваются топологически эквивалентньми.

В. Группы Ли и алгебры Ли. Пусть $G$ - связная односвязная вешественная група Ли. Всюду далее $1 \in G$ обозначает единичньй элемент, а $Z(G)$ - центр в $G$. $\bar{M} \subset G$ будет обозначать замыкание подмножества $M \subset G$, а $H_{0} \subset G$ - связную компоненту единицы замкнутого подмножества $H \subset G$, содержащего $1 \in G$.

Максимальная связная нормальная нильпотентная (соответственно, разрешимая) подгрупша называется нильрадикалом (соответственно, радикалом) в $G$. Имеет место разложение Леви в полупрямоепроизведение $G=L \cdot R$ радикала $R$ и максимальной полупростой подгрупшы $L$. Связная односвязная полупростая группа $L$ в свою очередь раскладывается в прямое произведение простых множителей. Для полупростой групшы $L$ через $K \subset L$ обозначим произведение всех компактных множителей, а через $S \subset L-$ всех некомпактных. Разложение $L=K \times S$ будем назьвать разложением на компактную и некомпактную части.

Пусть $\mathfrak{g}$ - алгебра Ли групшы $G$. Каждый элемент $x \in \mathfrak{g}$ алгебры Ли индуцирует однопараметрическую подгруппу $\exp (\mathbb{R} x) \subset G$ с единичным касательным вектором $x$.

\footnotetext{
${ }^{7}$ Данное определение соответствует термину "unique ergodicity". Если потребовать, чтобы поток вдобавок являлся минималшным, получится "strict ergodicity".
} 
Под присоединенным представлением $\mathrm{Ad}: G \rightarrow \operatorname{Aut}(\mathfrak{g})$ в данной работе мы будем понимать дифференциал сопряжения $h \rightarrow g^{-1} h g$, т.е. $\operatorname{Ad}_{g}(x)=\left.\frac{d g^{-1} \exp (t x) g}{d t}\right|_{t=0}$. Заметим, что $\operatorname{Ad}_{g} \operatorname{Ad}_{g^{\prime}}=\operatorname{Ad}_{g^{\prime} g}{ }^{8}$

Для любой замкнутой подгруппы $\Gamma \subset G$ левоинвариантная мера Хаара на $G$ индуцирует гладкую меру объема $\nu$ на левом однородном пространстве $\Gamma \backslash G$. Если мера $\nu$ является конечной и $G$-инвариантной относительно правого действия $G$, то $\Gamma \backslash G$ называется пространством конечного объема. Если $\Gamma \subset \Lambda \subset G$, то пространство $\Gamma \backslash G$ имеет конечньй объем $е$ пространства $\Lambda \backslash G$ и $\Gamma \backslash \Lambda$ имеют конечньй объем. Если $\Gamma$ дискретна и $\nu(\Gamma \backslash G)<\infty$, то мера $\nu$ автоматически является $G$-инвариантной и $\Gamma$ называется решеткой в $G$.

Подгруппа $\Gamma \subset G$ назьвается равномерной, если $\Gamma \backslash G$-компакт. Равномерная дискретная подгруппа всегда является решеткой. Известно, что полупростая группа Ли $G$ содержит как равномерные, так и неравномерные решетки. Любая решетка в разрешимой группе является равномерной, но не каждая даже нильпотентная группа Ли содержит хотя бы одну решетку. Решетки $\Gamma, \Gamma^{\prime} \subset G$ назьваются соизмеримыми, если пересечение $\Gamma \cap \Gamma^{\prime}$ имеет конечный индекс в $\Gamma$ и $\Gamma^{\prime}$. Решетка $\Gamma \subset G$ называется несократимой, если она не содержит нетривиальных нормальных в $G$ подгрупп (это эквивалентно тому, что пересечение $\Gamma \cap Z(G)$ тривиально).

Приведем несколько определений из теории алгебраических груп в той обшности, которая нам понадобится; подробнее см. [Hu] или $[\mathrm{VO}]$. Подмножество $H \subset S L(n, \mathbb{R})$ называется (вешественно-) алгебраическим или замкнутым по Зарисскому, если оно выделяется полиномиальным уравнением от матричных элементов с вещественньми коэффициентами. Такое определение задает на $S L(n, \mathbb{R})$ и на ее алгебраических подгруппах так назьваемую топологию Зарисского. Обычную топологию на $S L(n, \mathbb{R})$ будем называть евклидовой. 9 Алгебраическая группа имеет конечное число компонент связности относительно евклидовой топологии.

Наименьшая алгебраическая подгрупша $A(H) \subset S L(n, \mathbb{R})$, содержашая $H \subset$ $S L(n, \mathbb{R})$, называется алгебраической оболочкой или замыканием по Зарисскому групшы $H$ (при этом говорят, что $H$ плотна по Зарисскому в $A(H))$. Напомним, что матрица $g \in S L(n, \mathbb{R})$ называется унипотентной, если найдется натуральное $n$ такое, что $(g-E)^{n}=0$, где $E$ - единичная матрица (это равносильно тому, что все собственные значения $g$ равны 1$). g \in S L(n, \mathbb{R})$ называется полупростой матрицей, если над полем $\mathbb{C}$ она приводится к диагональному виду, и $\mathbb{R}$-диагонализуемой, если она приводится к диагональному виду над $\mathbb{R}$. Каждый элемент $g$ в алгебраической группе $H \subset S L(n, \mathbb{R})$ является произведением $g=g_{s} \times g_{u}$ коммутируюших полупростого и унипотентного элементов $g_{s}, g_{u} \in H$. Каждая абелева алгебраическая подгруппа $A \subset H$ из полупростых элементов раскладывается в произведение $A=T \times V$ тора $T$ и односвязной $\mathbb{R}$-диагонализуемой подгрупшы $V$.

Подгруппа $H \subset S L(n, \mathbb{R})$ назьвается унипотентной, если она целиком состоит из унипотентных элементов. Известно, что связные унипотентные подгрупшы в $S L(n, \mathbb{R})$, так же как и компактные, являются алгебраическими. Полупростая

\footnotetext{
${ }^{8}$ Такое определение Аd вызвано тем, что мы рассматриваем левые однородные пространства.

${ }^{9}$ Иногда ее назьвают хаусдорфовой, подчеркивая отличие от нехаусдорфовой топологии Зарисского.
} 
подгрупа в $S L(n, \mathbb{R})$ имеет конечный индекс в своей алгебраической оболочке, что по существу тоже позволяет рассматривать ее как алгебраическую группу. Произвольная полупростая групп $L$ проектируется с дискретньм ядром $Z(L)$ на алгебраическую подгрупу $\mathrm{Ad} L$ в группе $S L(\mathfrak{l})=S L(n, \mathbb{R})$ унимодулярных линейных преобразований своей алгебры Ли $\mathfrak{l}, \operatorname{dim}(\mathfrak{l})=n$. $\mathbb{R}-$ рангом полупростой группы Ли $L$ называют размерность максимальной $\mathbb{R}$-диагонализуемой подгруппы в $\operatorname{Ad} L$. Для примера: $\operatorname{rank}_{\mathbb{R}} S L(n, \mathbb{R})=n-1, \operatorname{rank}_{\mathbb{R}} S O(1, n)=1, \mathbb{R}$-ранг компактной групшы равен 0. Заметим, что компактная полупростая групп не содержит однопараметрических унипотентных подгруп и, напротив, простая некомпактная группа Ли порождается такими подгруппами.

Редуктивной подгруппой $H \subset S L(n, \mathbb{R})$ назьвается подгруппа с вполне приводимым действием на $\mathbb{R}^{n}$. Известно, что алгебраическая редуктивная подгруппа есть коммутативное произведение полупростой группы и абелевой групшы, состоящей из полупростых элементов.

Если подгрупа $H \subset S L(n, \mathbb{R})$ выделяется полиномиальным уравнением с рациональными коэффициентами, она называется $\mathbb{Q}$-определенной алгебраической групой. Если $H \subset S L(n, \mathbb{R})$ определена над $\mathbb{Q}$, то группа ее рациональных точек $H_{\mathbb{Q}}=H \cap S L(n, \mathbb{Q})$ плотна в $H . \quad$ Алгебраическая оболочка любой подгруппы $H \subset S L(n, \mathbb{Q})$ определена над $\mathbb{Q}$.

Пусть теперь $\rho: G \rightarrow S L(n, \mathbb{R})$ - любое конечномерное представление связной группы Ли $G$. Говорят, что подгруппа $H \subset G$ плотна по Зарисскому в представлении $\rho$, если алгебраические оболочки $\rho(H)$ и $\rho(G)$ совпадают. Представление называют унипотентным, если подгрупа $\rho(G) \subset S L(n, \mathbb{R})$ унипотентна. Хорошо известны следуюшие результаты (все они, если не оговорено противное, содержатся в книге [Rag] и обзоре [VGS]).

Теорема В.1 (Мальцев). Любая решетка в нильпотентной группе Ли $N$ плотна по Зарисскому во всех конечномерных унипотентных представлениях.

Теорема В.2 (Борель). Пусть $L=K \times S$ - разложение полупростой груnпь Ли на компактную и некомпактную части и решетка $\Gamma \subset L$ такова, что $L=\overline{\Gamma S}$. Тогда Г плотна по Зарисскому во всех конечномерных представлениgx L.

Эти два результата были распространены на обшие группы Ли следуюшим образом.

Tеорема В.3 (Дани, [Da80]). Пусть $\rho: G \rightarrow S L(n, \mathbb{R})$ - конечномерное представление связной группь Ли $G$ и Г-решетка в $G$. Тогда алгебраическая оболочка $\rho(\Gamma)$ содержит все унипотентные однопараметрические подгруппы в $\rho(G)$.

Один из приемов построения решеток в полупростых группах состоит в следуюшем.

Tеорема В.4 (Борель-Хариш-Чандра). Eсли $L \subset S L(n, \mathbb{R})$ является $\mathbb{Q}$-олределенной редуктивной алгебрачческой подгруппой без нетривиальных $\mathbb{Q}$-характеров $\chi: L \rightarrow \mathbb{R}^{*}$ (это заведомо так, если $L$ полупроста), то пересечение $L_{\mathbb{Z}}=L \cap S L(n, \mathbb{Z})$ является решеткой в $L$. 
Решетки $L_{\mathbb{Z}} \subset L=S K$ и $L_{\mathbb{Z}} K \cap S \subset S$ являются примерами так назьваемых арифметических решеток. Точнее: решетка $Г$ в полупростой групе Ли $L$ назьвается арифметической, если найдутся $\mathbb{Q}$-определенная группа $H \subset S L(n, \mathbb{R})$ и эпиморфизм $\rho: H \rightarrow \operatorname{Ad} L$ с компактным ядром такие, что группа $\rho\left(H_{\mathbb{Z}}\right)$ соизмерима с $\operatorname{Ad} \Gamma$.

Решетка Г в полупростой группе $S$ без компактных множителей назьвается неприводимой, если $\overline{\Gamma S^{\prime}}=G$ для любой связной нетривиальной нормальной подгрупшы $S^{\prime} \subset S$. Известно, что для любой решетки $\Gamma$ в полупростой группе $S$ без компактных множителей найдутся подгруппа конечного индекса $\Gamma^{\prime} \subset$ Г и разложение $S=\prod_{i=1}^{n} S_{i}$ в произведение нормальных подгруп такие, что $\Gamma^{\prime}=\prod_{i=1}^{n}\left(\Gamma^{\prime} \cap S_{i}\right)$ и $\Gamma^{\prime} \cap S_{i}$ является неприводимой решеткой в $S_{i}$ для каждого $i=1, \ldots, n$. Центральным результатом в теории арифметических решеток является

Теорема В.5 (Маргулис, см. [Ма90а]). Если Г - неприводимая решетка в полупростой әруппе $S$ без компактных множителей $и \operatorname{rank}_{\mathbb{R}} S \geqslant 2$, то Г арифметична.

Заметим, что первые примеры неарифметических решеток в $S O(1, n), n=3,4,5$, были построены Макаровым и Винбергом.

Нам понадобятся также несколько утверждений об устройстве решеток. Заметим, что если $\Gamma$ - решетка в $G$ и $H$ - связная нормальная подгруппа в $G$, то замкнутость произведения $\Gamma H$ равносильна тому, что пересечение $\Gamma \cap H$ является решеткой в $H$. Далее в теоремах В.6-В.9 группы Ли предполагаются связньми и односвязньми.

Теорема В.6 (Мостов). Если $\Gamma$-решетка в разрешимой группе Ли $R$ u $N \subset R$ - нильрадикал в $R$, то пересечение $\Gamma \cap N$ является решеткой в $N$.

Обобщением этого утверждения является

Tеорема B.7 (Старков, [St93]). Если $\Gamma$ - решетка в группе Ли G с разложсением Леви $G=L \cdot R$ и $N \subset R$ - нильрадикал в $G$, то пересечение $\Gamma \cap L N$ является решеткой в $L N$.

Доказательство опирается на одно утверждение из работы Витте [Wi87], из которого следует наш результат в случае $G=\overline{\Gamma S R}$, где $S \subset L$ - некомпактная часть $L$. Вообше, случай $G=\overline{\Gamma S R}$ является наиболее привлекательным с точки зрения устройства решетки Г. Дело в том, что имеет место

Tеорема В.8 (Витте, [Wi87]; Старков, [St87a]). Eсли $G=\overline{\Gamma S R}$, то пересечение $\Gamma \cap R$ является решеткой в радикале $R$ (следовательно, и пересечение $\Gamma \cap N$ является решеткой в нильрадикале $N)$.

Заметим, что в обшем случае пересечение решетки с радикалом или нильрадикалом может не являться там решеткой (простейший пример: $G=S O(3) \times \mathbb{R}$ и $\Gamma-$ решетка в цилиндре $S O(2) \times \mathbb{R}$, тривиально пересекаюшая $\mathbb{R})$. В самом общем случае имеет место важная

Теорема В.9 (Ауслендер). Если $\Gamma$ - решетка в группе Ли $G=L \cdot R$, то найдется тор $T \subset K \subset L$ такой, что пересечение $\Gamma \cap T R$ является решеткой в TR. 
С. Однопараметрические однородные потоки. Зафиксируем элемент $g \in G$ и рассмотрим линейный оператор $\mathrm{Ad}_{g}$ на алгебре Ли g. Относительно этого оператора комплексифицированная алгебра Ли $\mathfrak{g}_{\mathbb{C}}=\mathfrak{g} \bigotimes_{\mathbb{R}} \mathbb{C}$ имеет разложение на конечное число инвариантных корневых подпространств $\mathfrak{g}_{\mathbb{C}}^{\lambda}$, где

$$
\mathfrak{g}_{\mathbb{C}}^{\lambda}=\left\{x \in \mathfrak{g}_{\mathbb{C}}: \exists n:\left(\operatorname{Ad}_{g}-\lambda E\right)^{n} x=0\right\} .
$$

Так как $\operatorname{Ad}_{g}[x, y]=\left[\operatorname{Ad}_{g} x, \operatorname{Ad}_{g} y\right]$, то $\left[\mathfrak{g}_{\mathbb{C}}^{\lambda}, \mathfrak{g}_{\mathbb{C}}^{\theta}\right] \subset \mathfrak{g}_{\mathbb{C}}^{\lambda \theta}$. Для вещественного $\lambda$ положим $\mathfrak{g}^{\lambda}=\mathfrak{g}_{\mathbb{C}}^{\lambda} \cap \mathfrak{g}$, а для пары комплексно-сопряженных корней $\lambda$ и $\bar{\lambda}$ положим $\mathfrak{g}^{\lambda}=\mathfrak{g}^{\bar{\lambda}}=$ $\left(\mathfrak{g}_{\mathbb{C}}^{\lambda}+\mathfrak{g}_{\mathbb{C}}^{\bar{\lambda}}\right) \cap \mathfrak{g}$. Мы получили разложение $\mathfrak{g}=\sum_{\lambda} \mathfrak{g}^{\lambda}$ в сумму инвариантных относительно $\operatorname{Ad}_{g}$ подпространств. Определим

$$
\mathfrak{g}^{+}=\sum_{|\lambda|>1} \mathfrak{g}^{\lambda}, \quad \mathfrak{g}^{0}=\sum_{|\lambda|=1} \mathfrak{g}^{\lambda}, \quad \mathfrak{g}^{-}=\sum_{|\lambda|<1} \mathfrak{g}^{\lambda} .
$$

Легко видеть, что $\mathfrak{g}^{+}$и $\mathfrak{g}^{-}$являются нильпотентными подалгебрами, которые нормализуются подалгеброй $\mathfrak{g}^{0}$.

Пусть $G^{+}, Q, G^{-}$- аналитические подгрупшы в $G$, соответствуюшие подалгебрам $\mathfrak{g}^{+}, \mathfrak{g}^{0}, \mathfrak{g}^{-}$. Подгруппы $G^{+}$и $G^{-}$мы будем называть соответственно расширящейся и сжимаюшейся орисферическими подгруппами (по отношению к элементу $g$ ). Подгруппу $Q$ назовем нейтральной. Легко видеть, что

$$
G^{+}=\left\{h \in G: g^{n} h g^{-n} \rightarrow 1, n \rightarrow+\infty\right\}, \quad G^{-}=\left\{h \in G: g^{-n} h g^{n} \rightarrow 1, n \rightarrow+\infty\right\},
$$

причем обе подгруппы нильпотентны и нормализуются подгруппой $Q$. Подгруппа $U \subset G$, порожденная $G^{+}$и $G^{-}$, назьвается нормальной подгрупшой Ауслендера для элемента $g \in G$.

Элемент $g$ назовем частично-гиперболическим, если подгруппа $U=U(g)$ нетривиальна. В противном случае (если $Q=G$ ) элемент $g$ назовем квазиунипотентным. Если к тому же все собственные значения оператора $\operatorname{Ad}_{g}$ равны 1 , элемент $g$ назовем унипотентным. Если оператор $\mathrm{Ad}_{g}$ диагонализуем над $\mathbb{C}$, элемент $g$ назовем редуктивным или полупростым, а если он диагонализуем над $\mathbb{R}$, то $g$ назовем $\mathbb{R}$-диагонализуемым. ${ }^{10}$ Заметим, что одновременная редуктивность и унипотентность означают, что $g$ - элемент центра $Z(G)$ (это вытекает из связности $G$ ). Одновременная редуктивность и квазиунипотентность означают, что $\operatorname{Ad}_{g}$ лежит в компактной подгрупе из $\operatorname{Aut}(\mathfrak{g})$.

Нетрудно доказать, что нормальная подг руппа Ауслендера $U$ есть наименьшая среди всех нормальных подгрупп $H \subset G$ таких, что элемент $g H \in G / H$ является квазиунипотентным в группе $G / H$. Нормальной подгруппой Дани $I$ для элемента $g \in$ $G=L R$ назьвается наименьшая среди всех нормальных подгруп $H \subset G$ таких, что $g H \in G / H$ квазиунипотентен в $G / H$ и $g H R \in G / H R$ редуктивен в полупростой группе $G / H R$. Наконец, нормальной подгрушпй Мура $F$ для элемента $g \in G$ назьвается

\footnotetext{
${ }^{10}$ Вернее было бы говорить об Аd-унипотентности и Аd-редуктивности, чтобы не путать эти понятия с аналогичными терминами для алгебраических групп. Если будет возникать двусмысленность, мы так и будем поступать.
} 
наименьшая среди всех нормальных подгрупп $H \subset G$ таких, что $g H \in G / H$ одновременно квазиунипотентен и редуктивен в $G / H$.

Очевидно, что $U \subset I \subset F$. Если группа $G$ нильпотентна, то $U=I=1$. Если $G$ разрешима, то $U=I$. Если $G$ полупроста, то $I=F$.

Пусть теперь $g_{\mathbb{R}}$ - однопараметрическая подгруппа в $G$. Очевидно, что подгруппы $G^{+}, G^{-}, Q, U, I, F$ одни и те же для всех элементов $g_{t}, t \neq 0$, что позволяет определять их для $g_{\mathbb{R}}$. Редуктивность, квазиунипотентность и частичная гиперболичность также не зависят от выбора $g_{t} \in g_{\mathbb{R}}$. Подгруппа $g_{\mathbb{R}}$ называется унипотентной, если таковы все ее элементы.

Всюду далее $\Gamma$ обозначает решетку в $G$. Однородным потоком $\left(\Gamma \backslash G, g_{\mathbb{R}}\right)$, индуцированным подгруппой $g_{\mathbb{R}} \subset G$, называется правое действие $g_{\mathbb{R}}$ на левом однородном пространстве $\Gamma \backslash G$, снабженном $G$-инвариантной гладкой мерой объема $\nu$. Пусть $d-$ левоинвариантная метрика на $G$. Тогда для любого $h \in G^{-}$

$$
d\left(g_{t}, h g_{t}\right)=d\left(1, g_{-t} h g_{t}\right) \rightarrow 0, \quad t \rightarrow+\infty
$$

и поэтому орбиты $G^{-}$на $\Gamma \backslash G$ образуют сжимающееся слоение для потока $\left(\Gamma \backslash G, g_{\mathbb{R}}\right)$ в метрике на $\Gamma \backslash G$, индуцированной метрикой $d$ (причем скорость сжатия экспоненциальна). Аналогично, орбиты $G^{+}$на $\Gamma \backslash G$ образуют расширяюшееся слоение для нашего потока. Если хотя бы одно из этих слоений на $\Gamma \backslash G$ нетривиально, то и другое нетривиально (в силу сохранения меры объема на $\Gamma \backslash G$ ) и наш поток является равномерно частично-гиперболическим. ${ }^{11}$ Для квазиунипотентного потока скорость разбегания близких траекторий не выше полиномиальной.

Нормальные подгруппы $U \subset I \subset F$ играют центральную роль для изучения эргодических свойств потока $\left(\Gamma \backslash G, g_{\mathbb{R}}\right)$ относительно конечной $G$-инвариантной меры $\nu$.

Tеорема C.1 (феномен Маутнера; Мур, [Mo80]). 1) Пусть $\rho: G \rightarrow U(\mathscr{H})-$ унитарное представление в гильбертовом пространстве $\mathscr{H} u h \in \mathscr{H}$ - такой элемент, что $\rho\left(g_{\mathbb{R}}\right) h \equiv h$. Тогда $\rho(F) h \equiv h$.

2) Поток $\left(\Gamma \backslash G, g_{\mathbb{R}}\right)$ является эргодическим $\Longleftrightarrow$ поток $\left(\overline{\Gamma F} \backslash G, g_{\mathbb{R}}\right)$ является

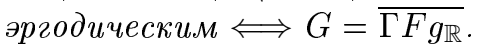

Красивый способ доказательства этой важной теоремы элементарными методами предложен в [Ma91b]; подробности см. в [MТ96]. Вторая часть теоремы вьводится из первой, если рассмотреть непрерьвное унитарное представление $G$ в $L^{2}(\Gamma \backslash G, \nu)$, даваемое формулой $\rho(g) f(x)=f(x g), f \in L^{2}(\Gamma \backslash G, \nu), x \in \Gamma \backslash G, g \in G$. Как следствие можно заметить, что топологически транзитивный однородный поток на пространстве конечного объема является эргодическим. В самом деле, если $G=\overline{\Gamma c g_{\mathbb{R}}}$, то $G=\overline{\Gamma c g_{\mathbb{R}}} c^{-1}$, и поэтому поток $\left(\Gamma \backslash G, c g_{\mathbb{R}} c^{-1}\right)$ эргодичен. Но этот поток метрически и топологически сопряжен с исходным потоком посредством отображения $\alpha(\Gamma h)=\Gamma h c^{-1}$. Заметим также, что $\left(\overline{\Gamma F} \backslash G, g_{\mathbb{R}}\right)$ является равномерно непрерьвньм факторпотоком исходного потока (и среди однородных факторов с таким свойством является максимальным).

\footnotetext{
${ }^{11}$ Пожалуй, впервые теория частично-гиперболических потоков для изучения однородных действий была применена Степиньм [Ste69].
} 
ТеОРема C.2 (Дани, [Da77]). Поток $\left(\Gamma \backslash G, g_{\mathbb{R}}\right)$ является перемешивающим $\Longleftrightarrow G=\overline{\Gamma I}$.

Теорема C.3 (Дани, [Da76,77]). Поток $\left(\Gamma \backslash G, g_{\mathbb{R}}\right)$ имеет положительную энтропию $\Longleftrightarrow$ он является частично-гиперболическим (т.е. $U \neq 1$ ); и является $K$-потоком $\Longleftrightarrow G=\overline{\Gamma U}$ (т.е. $\left(\overline{\Gamma U} \backslash G, g_{\mathbb{R}}\right)$ является максимальным факторпотоком нулевой әнтропии).

Заметим, что положительность энтропии для частично-гиперболического однородного потока нетрудно установить непосредственно. Ее тривиальность для квазиунипотентного потока в компактном случае следует из оценки Кушниренко [Ku]. Точная формула для энтропии [Da77] (которой мы не будем пользоваться) такая:

$$
e\left(g_{1}\right)=\sum_{\left|\lambda_{i}\right|>1} \log \left|\lambda_{i}\right|,
$$

где $\lambda_{1}, \ldots, \lambda_{n}$ - собственные числа оператора $\operatorname{Ad}_{g_{1}}$ на $\mathfrak{g}$. В компактном случае она была получена Боуэном [Во71] и следует из общей формулы Миллионщикова-Песина [Mi76], [Ре] для энтропии гладкой динамической системы.

В частности, унипотентные потоки на факторпространстве простой групшы Ли по решетке являются перемешиваюшими, а частично-гиперболические к тому же и $K$-потоками.

Далеко не каждое пространство конечного объема несет на себе эргодический однородный поток. Оказывается, существование такого потока накладьвает сильные ограничения на структуру пространства $\Gamma \backslash G$ :

Tеорема C.4 (Витте, [Wi87]; Старков, [St87a]). Ecли на $\Gamma \backslash G$ существует эргодический однородный поток, то $G=\overline{\Gamma S R}$, и потому пересечение $\Gamma \cap R$ является (равномерной) решеткой в $R$.

Существование перемешивающего потока сказьвается на строении пространства $\Gamma \backslash G$ еше сильнее:

Теорема С.5 (Витте, [Wi87]). Если на $\Gamma \backslash G$ действует однородный перемешивающий поток, то $G=L N$, причем решетка Г является плотной по Зарисскому в любом конечномерном представлении, унипотентном на $N$.

Заметим,что этот результат (a posteriori) нетрудно вьвести из теорем В.7, C.4, В.1 и В.2.

Обозначим через $G_{\infty} \subset G$ наименьшую нормальную подгруппу, содержащую подгруппу Леви $L \subset G$. Нам понадобится также следуюший критерий наличия перемешивающих потоков на $\Gamma \backslash G$.

Tеорема C.6 (Дани, [Da77]; Старков, [St95c]). Ecли $G \neq \overline{\Gamma G_{\infty}}$, то на $\Gamma \backslash G$ нет перемешивающих потоков. Если $G=\overline{\Gamma G_{\infty}}$, то любой эргодический поток на $\Gamma \backslash G$ является перемешивающим.

Первая часть этого результата легко следует из того факта, что на факторпространстве $\overline{\Gamma G_{\infty}} \backslash G$ транзитивно действует радикал $R$. Но разрешимое однородное пространство всегда однородным образом расслаивается над тором (см., к примеру, В.5) и поэтому не несет на себе перемешиваюших однородных потоков. 
Предположим теперь, что поток $\left(\Gamma \backslash G, g_{\mathbb{R}}\right)$ неэргодичен. Тогда в силу эргодического разложения, найденного автором в [St83], пространство $\Gamma \backslash G$ разбивается на гладкие подмногообразия $E_{h}\left(g_{\mathbb{R}}\right)=\overline{\Gamma h I g_{\mathbb{R}}}$, где $h \in G$, причем на каждом $E_{h}\left(g_{\mathbb{R}}\right)$

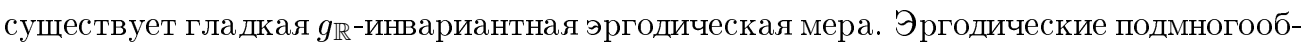
разия не обязаны быть однородными подпространствами в $\Gamma \backslash G$, т.е. в $G$ может не быть подгруппы с транзитивным действием на $E_{h}\left(g_{\mathbb{R}}\right)$ (впрочем, поток $\left(E_{h}\left(g_{\mathbb{R}}\right), g_{\mathbb{R}}\right)$ конечнолистно накрывается некоторым эргодическим однородньм потоком [St89]).

D. Унипотентные потоки. Если наш однородный поток индуцирован унипотентной подгруппой $u_{\mathbb{R}}$, эргодическое разложение устроено весьма просто: $\Gamma \backslash G$ разбивается на однородные эргодические подпространства. Чтобы доказать однородность $E_{1}\left(u_{\mathbb{R}}\right)=\overline{\Gamma I u_{\mathbb{R}}}$, достаточно рассмотреть случай, когда нормальная подгруппа Дани $I$ тривиальна. Так как $u_{\mathbb{R}} \subset S \cdot N$, где $N$ - нильрадикал в $G$, то из $I=1$ следует $u_{\mathbb{R}} \subset N \subset G$. Пусть $R^{\prime}=T \cdot R$-подгруппа из теоремы В.9 Ауслендера, которая пересекается с Г по решетке. Осталось применить следуюший результат к унипотентному потоку $\left(\Gamma \cap R^{\prime} \backslash R^{\prime}, u_{\mathbb{R}}\right)$.

Tеорема D.1 (Старков, $[\mathrm{St} 84])$. Eсли $\left(\Gamma \backslash G, u_{\mathbb{R}}\right)$-унипотентный поток на компактном разрешимом однородном пространстве, то замыкание $\overline{\Gamma u_{\mathbb{R}}} \subset \Gamma \backslash G$ является компактным минимальным однородным подпространством в $Г \backslash G$.

Заметим, что этот результат вьводится из теоремы Ауслендера [AGH] о минимальности эргодического потока на нильпотентном однородном пространстве.

Однородность остальных эргодических подмногообразий $E_{g}\left(u_{\mathbb{R}}\right)$ вытекает из очевидной формулы $E_{g}\left(u_{\mathbb{R}}\right)=E_{1}\left(g u_{\mathbb{R}} g^{-1}\right) g$. Так как все эргодические компонентыв разрешимом случае являются минимальными, то (в общем случае) множества $E_{g}\left(u_{\mathbb{R}}\right)$ для разных $g \in G$ либо не пересекаются, либо совпадают. Поскольку почти все орбиты эргодического потока являются всюду плотными, нетрудно доказать, что почти всегда $\overline{\Gamma g u_{\mathbb{R}}}=E_{g}\left(u_{\mathbb{R}}\right)$. Итак, имеет место

Tеорема D.2 (Старков, [St83]). По отношению $\kappa$ унипотентному потоку $\left(\Gamma \backslash G, u_{\mathbb{R}}\right)$ пространство разбивается на однородные подпространства Гg $P_{g}$ с конечной $P_{g}$-инвариантной $u_{\mathbb{R}}$-эргодической мерой. Для почти всех $g \in G$ пространство $\Gamma g P_{g}$ совпадает с замыканием орбиты $\Gamma g u_{\mathbb{R}}$.

Итак, эргодические компоненты унипотентных потоков являются однородными подпространствами конечного объема. Это позволяет при изучении унипотентных потоков сосредоточиться на эргодическом случае. Другой способ редукции к эргодическому случаю заключается в следуюшем.

Teоpema D.3 (Шах, [Sh91]). Пусть $H_{g}$ - наименьшая подгруппа в $G$ такая, что $u_{\mathbb{R}} \subset H_{g}$ и орбита $\Gamma g H_{g}$ замкнута. Тогда $\Gamma g H_{g}$ имеет конечную $H_{g}$-инвариантную $u_{\mathbb{R}}-$ эргодическую меру.

Заметим, что подпространства $\Gamma g H_{g}$ в теореме D.3 могут быть вложены одно в другое и поэтому, вообше говоря, в топологическом смысле не образуют разбиения пространства $\Gamma \backslash G$. Подгрупша $H_{g}$ всегда является подгруппой в $P_{g}$ (хотя для почти всех $g \in G$ они совпадают).

Дадим теперь критерий эргодичности для подпотока. 
TeOPema D.4 (Шаx, [Sh91]). Пусть $u_{\mathbb{R}} \subset H \subset G$, пространство $Г H \subset \Gamma \backslash G$ замкнуто и имеет конечную $H$-инвариантную меру, а $F \subset H$ - наименьшая нормальная подгруппа в $H$, содержащая унипотентную подгруппу $u_{\mathbb{R}}$. Тогда поток $\left(\Gamma H, u_{\mathbb{R}}\right)$ эргодичен $\Longleftrightarrow \overline{\Gamma F}=\Gamma H$. В случае эргодичности решетка $\Gamma \cap H$ плотна в $H$ относительно $\mathrm{Ad}_{G}$-представления.

Для доказательства этого результата заметим, что $F$ является подгруппой Маутнера для $u_{\mathbb{R}} \subset H$ и к тому же порождена однопараметрическими унипотентными подгруппами. Теперь утверждение о плотности следует из теоремы В.3.

К этому результату близко примыкает

Tеорема D.5 (Витте, [Wi85]). Eсли поток $\left(\Gamma \backslash G, u_{\mathbb{R}}\right)$ унипотентен и әргодичен, то $G=L N$, причем решетка Г является плотной по Зарисскому в любом конечномерном представлении, унипотентном на $N$.

Рассмотрим правое действие $S L(n, \mathbb{R})$ на $\mathbb{R}^{n}$. Основным связуюшим звеном между теорией чисел и теорией однородных потоков служит следуюший критерий Малера.

Teорема D.6 (см. [Rag]). Последовательность $S L(n, \mathbb{Z}) g_{i}$ уходит на бесконечность в $S L(n, \mathbb{Z}) \backslash S L(n, \mathbb{R}) \Longleftrightarrow$ найдется последовательность ненулевых векторов $e_{i} \in \mathbb{Z}^{n}$ такая, что е e $_{i} \rightarrow 0, i \rightarrow \infty$.

Сосредоточимся теперь на траекториях унипотентных потоков. Как следует из теоремы D.2, замыкание почти каждой траектории является однородньм пространством конечного объема. Что можно сказать об остальных траекториях? Частичный ответ дает весьма нетривиальньй результат, которьй получил название леммы Маргулиса.

Теорема D.7 (Маргулис, [Ма71]). Унипотентный поток на пространстве конечного оббема не имеет дивергентных полуорбит.

На самом деле Маргулис доказал это утверждение для унипотентных потоков на $S L(n, \mathbb{Z}) \backslash S L(n, \mathbb{R})$. Для полупростых групा Ли $\mathbb{R}$-ранга 1 оно было доказано Дани [Da84]. Случай произвольной полупростой групшы Ли $G$ сводится к рассмотренным двум благодаря теореме арифметичности Маргулиса (см. [Da86b]). Обший случай рассматривается так: ввиду того, что эргодические компоненты унипотентного потока всегда однородны, достаточно рассмотреть эргодический поток $\left(\Gamma \backslash G, u_{\mathbb{R}}\right)$. Но в силу теоремы С.4 этот поток компактно накрывает поток $\left(q(\Gamma) \backslash L, q\left(u_{\mathbb{R}}\right)\right)$ на полупростом однородном пространстве (здесь $q: G=L \cdot R \rightarrow L$ - естественная проекция).

Существенным усилением леммы Маргулиса является следующий результат, согласно которому унипотентная траектория не только бесконечно часто возврашается в некоторьй компакт, но и проводит в нем бо́льшую часть времени.

Teоpema D.8 (Дани, [Da86b]). Для любого унипотентного потока $\left(\Gamma \backslash G, u_{\mathbb{R}}\right)$, точки $x \in \Gamma \backslash G$ и $\varepsilon>0$ найдется компакт $C \subset \Gamma \backslash G$ такой, что

$$
l\left\{t \in[0, T]: x u_{t} \in C\right\} \geqslant(1-\varepsilon) T \text { для всех } T \geqslant 0 .
$$

3 десь $l$ обозначает лебегову меру на $\mathbb{R}$. Мы продемонстрируем доказательство теорем D.7, D.8 на простейшем примере: $G=S L(2, \mathbb{R}), \Gamma=S L(2, \mathbb{Z})$. Пусть

$$
D_{s}=\left\{x \in \mathbb{R}^{2}:\|x\|<s\right\}, \quad K_{s}=\left\{g \in G: \mathbb{Z}^{2} g \cap D_{s}=0\right\} .
$$


По критерию Малера $K_{s}$ является компактом в $\Gamma \backslash G$. Вектор $e \in \mathbb{Z}^{2}$ назовем примитивным, если $\alpha e \in \mathbb{Z}^{2} \Longleftrightarrow \alpha \in \mathbb{Z}$. Пусть $\left\{e_{i}\right\}$ - максимальньй набор попарно неколлинеарных примитивных векторов (т.е. $\mathbb{Z} e_{i} \cap \mathbb{Z} e_{j}=0, i \neq j$, и $\bigcup_{i} \mathbb{Z} e_{i}=\mathbb{Z}^{2}$ ). Возьмем произвольную унипотентную подгруппу $\left\{u_{t}\right\} \subset G$ и зафикисируем $g \in G, \varepsilon>0$. Заметим, что решетка $\mathbb{Z}^{2} g$ для $g \in G$ имеет плошадь фундаментального параллелограмма равную 1 . Пусть

$$
A_{i}(T)=\left\{t \in[0, T]: e_{i} g u_{t} \in D_{\varepsilon}\right\}, \quad B_{i}(T)=\left\{t \in[0, T]: e_{i} g u_{t} \in D_{1}\right\}
$$

и $a_{i}(T)=l\left(A_{i}(T)\right), b_{i}(T)=l\left(B_{i}(T)\right)$. Так как точка $e_{i} g u_{t}$ движется по прямой с постоянной скоростью, $a_{i}(T) \leqslant 2 \varepsilon b_{i}(T)$ для достаточно больших $T$. Отсюда

$$
\limsup _{T \rightarrow \infty} \frac{\sum_{i} a_{i}(T)}{\sum_{i} b_{i}(T)} \leqslant 2 \varepsilon .
$$

Основное замечание: $B_{i}(T) \cap B_{j}(T)=\varnothing, i \neq j$ (иначе $e_{i}$ и $e_{j}$ порождали бы решетку с плошадью фундаментального параллелограмма менее 1$)$. Поэтому $\sum_{i} b_{i}(T) \leqslant T$. Теперь, поскольку $\sum_{i} A_{i}(T)=\left\{t \in[0, T]:\right.$ Гgu $\left.t \notin K_{\varepsilon}\right\}$, мы вьводим $l(t \in[0, T]$ : $\left.\Gamma g u_{t} \in K_{\varepsilon}\right) \geqslant(1-4 \varepsilon) T$ для достаточно больших $T$.

Для $n \geqslant 3$ основная трудность не в том, что точка $e_{i} g u_{t}$ движется по полиномиальному закону, а в том, что множества $B_{i}(T)$ не обязаны быть дизъюнктными (и поэтому приходится применять довольно сложное индуктивное рассуждение).

Пусть $\mu$ - локально-конечная эргодическая мера для унипотентного потока. Тогда компакт $C$ для каждой точки $x \in \Gamma \backslash G$ можно выбрать таким, что $\mu(C)>0$. Взяв теперь всюду положительную функцию $f \in L^{1}(\Gamma \backslash G, \mu)$ и применив эргодическую теорему Биркгофа-Хинчина, в результате усреднения мы почти всюду должны получить положительную константу $f^{*}$. Отсюда вытекает

Tеорема D.9 (Дани, [Da84]). Локально-конечная эргодическая мера для унипотентного потока на пространстве конечного облема является конечной.

Этот результат можно обобщить на более широкий класс подгрупп. Для связных унипотентных подгрупп $H \subset G$ конечность локально-конечных мер доказана Дани [Da84]. Имеется и более общий результат:

Tеорема D.10 (Маргулис, [Mа86]). Если $H=S_{H} N_{H}$, әде $N_{H}$ - унипотентна в $G$ и нормальна в $H$ и $S_{H}$ - полупростая группа без компактных множителей, то любая локально-конечная әргодическая мера на $\Gamma \backslash G$ является конечной.

Заметим, что из этого результата можно вывести теорему В.4 Бореля-Хариш-Чандры для полупростых групп. В самом деле, пусть $H \subset S L(n, \mathbb{R})$ - полупростая $\mathbb{Q}$-определенная группа без компактных множителей. Тогда произведение $S L(n, \mathbb{Z}) H$ замкнуто и поэтому мера Хаара на $H$ индуцирует $H$-эргодическую локально-конечную меру на $S L(n, \mathbb{Z}) \cap H \backslash H$. Она обязана быть конечной и, следовательно, $H$ пересекает $S L(n, \mathbb{Z})$ по решетке.

Наконец, заметим, что любая подгруппа $H \subset G$, порожденная однопараметрическими унипотентными в $G$ подгруппами, удовлетворяет условиям теоремы D.10 (см., например, [Sh91]). Следовательно, любая локально-конечная $H$-эргодическая мера на $\Gamma \backslash G$ является конечной. 


\section{§1. Поверхности постоянной отрицательной кривизны}

1. Напомним вкратце основные факты из гиперболической геометрии. Плоскость Лобачевского $\mathbb{H}^{2}$ постоянной отрищательной кривизны $-\lambda^{2}$ в модели Пуанкаре является комплексной полуплоскостью $y=\operatorname{Im}(z)>0$ с метрикой $d l^{2}=\frac{d x^{2}+d y^{2}}{\lambda^{2} y^{2}}$. Прямая $y=0$, дополненная бесконечно удаленной точкой, назьвается абсолютом. Группа Iso $\left(\mathbb{H}^{2}\right)$ всех изометрий $\mathbb{H}^{2}$ состоит из комбинаций отражения $s(z)=-\bar{z}$ и дробно-линейных преобразований

$$
z \rightarrow \frac{a z+b}{c z+d}, \quad a d-b c=1
$$

Групша Iso ${ }^{+}\left(\mathbb{H}^{2}\right)$ всех дробно-линейных преобразований состоит из всех изометрий $\mathbb{H}^{2}$, сохраняюших ориентацию, и является связной компонентой групшы Iso $\left(\mathbb{H}^{2}\right)$. Поэтому если

$$
g=\left(\begin{array}{ll}
a & b \\
c & d
\end{array}\right) \in S L(2, \mathbb{R}) \text { и } f_{g}(z)=\frac{a z+b}{c z+d},
$$

то соответствие $g \rightarrow f_{g}$ задает эпиморфизм $f: S L(2, \mathbb{R}) \rightarrow \mathrm{Iso}^{+}\left(\mathbb{H}^{2}\right)$ с ядром $\mathbb{Z}_{2}=$ $\{ \pm 1\} \in S L(2, \mathbb{R})$ (или, что то же самое, изоморфизм $\hat{f}: \operatorname{PSL}(2, \mathbb{R})=S L(2, \mathbb{R}) / \mathbb{Z}_{2} \rightarrow$ $\left.\mathrm{IsO}^{+}\left(\mathbb{H}^{2}\right)\right)$.

Далее $G=S L(2, \mathbb{R}), B$ - двумерная разрешимая подгруппа в $G$ всех верхнетреугольных матриц, $K=S O(2, \mathbb{R})$ - максимальная компактная подгруппа в $G$.

Любую точку $z=x+i y \in \mathbb{H}^{2}$ можно представить в виде

$$
z=f_{g_{z}}\left(z_{0}\right), \quad \text { где } z_{0}=i, \quad g_{z}=\left(\begin{array}{cc}
\sqrt{y} & x / \sqrt{y} \\
0 & 1 / \sqrt{y}
\end{array}\right) \in B .
$$

В частности, действия $B$ и, тем более, $G$ на $\mathbb{H}^{2}$ транзитивны. Стабилизатор точки $z_{0}=i$ состоит в точности из подгрупшы $K=S O(2, \mathbb{R})$. Это означает, что отображение $\varphi(g K)=f_{g}(i)$ устанавливает диффеоморфизмы

$$
\varphi: G / K \rightarrow \mathbb{H}^{2} \quad \text { и } \hat{\varphi}: \operatorname{PSL}(2, \mathbb{R}) / P S O(2, \mathbb{R}) \rightarrow \mathbb{H}^{2}
$$

Метрика $d l$ с точностью до множителя совпадает с метрикой, полученной из $G$-левоинвариантной и $K$-правоинвариантной метрики Киллинга $d$ на $G$.

Обозначим через $S \mathbb{H}^{2}$ расслоение единичных касательных векторов над $\mathbb{H}^{2}$. Элементами $S \mathbb{H}^{2}$ служат всевозможные пары $(z, v)$, где $z \in \mathbb{H}^{2}$ и $v \in S_{z} \mathbb{H}^{2}$ - единичный касательньй вектор к $\mathbb{H}^{2}$ в точке $z$. Дифференциал $d f_{g}$ любого действия $f_{g}$ задает диффеоморфизм $S \mathbb{H}^{2} \rightarrow S \mathbb{H}^{2}$. Поэтому мы получаем индуцированное действие $d f$ групшы $G$ на $S \mathbb{H}^{2}$.

Дифференциал $d f_{k}$ для любого элемента $k=\left(\begin{array}{rr}\cos \varphi & \sin \varphi \\ -\sin \varphi & \cos \varphi\end{array}\right) \in K$ задает поворот единичной касательной окружности $S^{1}=S_{i} \mathbb{H}^{2}$ на угол $2 \varphi$. Поэтому ядром индуцированного действия $d f$ на $S \mathbb{H}^{2}$ является снова $\mathbb{Z}_{2} \subset G$. 
Зафиксируем единичный касательный вектор $e=(0,1)$ в точке $z_{0}=i$. Тогда любая пара $(z, v)$ получается из пары $(i, e)$ путем подходяшей подкрутки $d f_{k}, k \in K$, единичной окружности в $S_{z_{0}} \mathbb{H}^{2}$ и последуюшего переноса $d f_{g_{z}}$ точки $i$ в точку $z$. Следовательно, отображение $F(g)=d f_{g}(i, e)$ задает накрытие $F: G \rightarrow S \mathbb{H}^{2}$ со слоем $\mathbb{Z}_{2}$ и одновременно диффеоморфизм $\widehat{F}: P S L(2, \mathbb{R}) \rightarrow S \mathbb{H}^{2}$. Попутно мы установили, что $G=B K=K B$. Заметим, что если $g \in \mathrm{Iso}^{+}\left(\mathbb{H}^{2}\right)$, то отображению $d f_{g}: S \mathbb{H}^{2} \rightarrow S \mathbb{H}^{2}$ соответствует левый сдвиг

$$
L_{g}: P S L(2, \mathbb{R}) \rightarrow P S L(2, \mathbb{R}), \quad L_{g}(h)=g h, \quad h \in P S L(2, \mathbb{R})
$$

(иначе говоря, $d f_{g} \circ \widehat{F}=\widehat{F} \circ L_{g}$ ). Отражению $s: z \rightarrow-\bar{z}$ соответствует внешний автоморфизм $\Sigma$ групшы $P S L(2, \mathbb{R}), \Sigma(h)=\sigma h \sigma^{-1}$, где $\sigma=\left(\begin{array}{rr}1 & 0 \\ 0 & -1\end{array}\right)$. Таким образом, $\Sigma\left(\left(\begin{array}{ll}a & b \\ c & d\end{array}\right)\right)=\left(\begin{array}{cc}a & -b \\ -c & d\end{array}\right)$

2. Известно, что из любой точки $z \in \mathbb{H}^{2}$ в направлении любого вектора $v \in S_{z} \mathbb{H}^{2}$ исходит ровно одна геодезическая линия. Задав операцию параллельного переноса касательных векторов при движении вдоль нее, мы получим однопараметрический поток $g_{t}$ на $S \mathbb{H}^{2}$, называемый геодезическим.

Легко проверить, что кривая $g_{t}(i, e)=i e^{\lambda t}$ задает геодезическую линию в $\mathbb{H}^{2}$, проходящую через $z_{0}=i$ в направлении вертикального вектора $e \in S_{z_{0}} \mathbb{H}^{2}$ с единичной скоростью. Поскольку любая изометрия переводит геодезическую в геодезическую и $G$ действует на $S \mathbb{H}^{2}$ транзитивно, то все геодезические исчерпываются кривыми вида $f_{g}\left(i e^{\lambda t}\right)$. Геометрически они представляют собой полуокружности (или полупрямые), ортогонально подходяшие к абсолюту $y=0$. Пусть

$$
A_{t}=\left(\begin{array}{cc}
e^{\lambda t / 2} & 0 \\
0 & e^{-\lambda t / 2}
\end{array}\right), \quad H_{t}=\left(\begin{array}{ll}
1 & 0 \\
t & 1
\end{array}\right), \quad U_{t}=\left(\begin{array}{ll}
1 & t \\
0 & 1
\end{array}\right)
$$

Тогда $i e^{\lambda t}=f_{A_{t}}(i)$, и поэтому любая геодезическая линия в $\mathbb{H}^{2}$ имеет вид $f_{g A_{t}}(i)$. Таким образом, $g_{t}(z, v)=d f_{g A_{t}}(i, e)$, где $d f_{g}(i, e)=(z, v)$, и поэтому $g_{t}$ соответствует правому действию $A_{t}$ на $G$.

Заметим, что подгрупшы $A, U, H \subset G$ связаны соотношениями

$$
A_{-t} U_{s} A_{t}=U_{e^{-\lambda t} s}, \quad A_{-t} H_{s} A_{t}=H_{e^{\lambda t} s},
$$

и поэтому $U$ и $H$ - соответственно сжимаюшаяся и расширяюшаяся орисферические подгрупшы для $A$. Поскольку для левоинвариантной метрики $d$ в $G$ имеем

$$
\begin{aligned}
d\left(g A_{t}, g U_{s} A_{t}\right) & =d\left(g A_{t}, g A_{t} A_{-t} U_{s} A_{t}\right)=d\left(1, A_{-t} U_{s} A_{t}\right) \\
& =d\left(1, U_{e^{-\lambda t} s}\right) \rightarrow 0, \quad t \rightarrow+\infty
\end{aligned}
$$

то правые орбиты $U$ в $G$ образуют сжимающееся слоение для правого действия $A$ на $G$. Следовательно, кривые $d f_{g U_{s}}(i, e)$ в $S \mathbb{H}^{2}$ образуют сжимающееся слоение $\mathscr{U}$ для геодезического потока на $S \mathbb{H}^{2}$. Они назьваются сжимающимися орициклами 
и геометрически представляют собой окружности, касающиеся абсолюта (или горизонтальные прямые, если $v=(0,1))$. Точнее, сжимающийся орицикл, заданный парой $(z, v) \in S \mathbb{H}^{2}$, представляет собой такую окружность (возможно бесконечного радиуса), проходящую через $z \in \mathbb{H}^{2}$ и касающуюся абсолюта, что вектор $v$ является ее внутренней нормалью.

Аналогично, расширяюшееся орициклическое слоение $\mathscr{H}$ в $S \mathbb{H}^{2}$ образовано кривыми $d f_{g H_{s}}(i, e)$. Расширяюшийся орицикл, заданный парой $(z, v) \in S \mathbb{H}^{2}$, является окружностью, проходящей через $z \in \mathbb{H}^{2}$ и касающейся абсолюта, причем вектор $v$ является ее внешней нормалью.

Итак, геодезический поток $g_{t}$ на трехмерном многообразии $S \mathbb{H}^{2}$ обладает расширяюшимся и сжимаюшимся слоениями и потому является аносовским. Оба слоения $\mathscr{U}$ и $\mathscr{H}$ в данном случае имеют естественную параметризацию временем $s \in \mathbb{R}$ и представляют, таким образом, однопараметрические потоки на $S \mathbb{H}^{2}$. Заметим, что такая параметризация является равномерной: геодезический поток за время $t>0$ сжимает слои $\mathscr{U}$ в $e^{\lambda t}$ раз и во столько же раз растягивает слои $\mathscr{H}$. Параметризация каждого орищикла в $S \mathbb{H}^{2}$ длиной его проекции в $\mathbb{H}^{2}$ отличается от параметризации по времени на постоянный множитель $\lambda$.

3. Напомним, что фуксовой группой назьвается любая дискретная подгруппа $\Gamma \subset$ $\mathrm{Iso}^{+}\left(\mathbb{H}^{2}\right)$. Произвольная гладкая ориентируемая поверхность $M$ постоянной кривизны $k(M)=-\lambda^{2}$ получается как факторпространство $\mathbb{H}^{2}$ с метрикой кривизны $-\lambda^{2}$ вдоль орбит действия некоторой фуксовой группы, не имеющей элементов конечного порядка (т.е. элементов, сопряженных элементам из $P S O(2, \mathbb{R})) .{ }^{12}$ Следовательно,

$$
M=\Gamma \backslash \mathbb{H}^{2}=\Gamma \backslash P S L(2, \mathbb{R}) / P S O(2, \mathbb{R}) \text { и } S M=\Gamma \backslash S \mathbb{H}^{2}=\Gamma \backslash P S L(2, \mathbb{R}) .
$$

С таким же успехом можно считать, что $M=\widetilde{\Gamma} \backslash G / K$ и $S M=\widetilde{\Gamma} \backslash G$, где $\widetilde{\Gamma} \subset G-$ прообраз Г при накрытии $S L(2, \mathbb{R}) \rightarrow P S L(2, \mathbb{R})$ (причем в $\widetilde{\Gamma}$ нет элементов конечного порядка кроме \pm 1$)$.

Потоки $g_{t}, u_{t}$ и $h_{t}$ на $S \mathbb{H}^{2}$ накрьвают соответствуюшие потоки на $S M$ (которые мы будем обозначать так же). Итак, геодезический поток $g_{t}$ на $S M$ есть не что иное, как однородный поток $\left(\Gamma \backslash G, A_{t}\right)$. Орициклические потоки $u_{t}$ и $h_{t}$ на $S M$ соответствуют однородным потокам $\left(\Gamma \backslash G, U_{t}\right)$ и $\left(\Gamma \backslash G, H_{t}\right)$.

Риманова метрика на $M$ индуцирует гладкую меру объема vol. С другой стороны, $S M=\Gamma \backslash G$ несет на себе гладкую $G$-инвариантную меру $\nu$, индуцированную биинвариантной мерой Хаара на $G$ и называемую мерой Лиувилля. При этом $\operatorname{vol}(M)<\infty$ тогда и только тогда, когда $\nu(S M)<\infty$, т.е. Г является решеткой в $G$. В этом случае меру Лиувилля $\nu$ мы будем нормировать условием $\nu(S M)=1$.

Предположим, что $\theta: M_{1} \rightarrow M_{2}$ есть изометрия двух ориентируемых поверхностей постоянной отрицательной кривизны $k\left(M_{1}\right)=k\left(M_{2}\right)$. Тогда $M_{i}=\Gamma_{i} \backslash \mathbb{H}^{2}$, где $\Gamma_{i} \subset \mathrm{Iso}^{+}\left(\mathbb{H}^{2}\right), i=1,2$, и $\theta$ поднимается до изометрии $\tilde{\theta}: \widetilde{M}_{1} \rightarrow \widetilde{M}_{2}$. Но $\widetilde{M}_{1}=\widetilde{M}_{2}=$ $\mathbb{H}^{2}$ и поэтому $\tilde{\theta} \in \operatorname{Iso}\left(\mathbb{H}^{2}\right)$ - элемент, переводяший $\Gamma_{1}$-орбиту любой точки $z \in H$ в $\Gamma_{2}$-орбиту точки $\tilde{\theta}(z), z \in \mathbb{H}^{2}$. Следовательно, $\Gamma_{2}=\tilde{\theta} \Gamma_{1} \tilde{\theta}^{-1}$. Если $\tilde{\theta} \in \operatorname{Iso}^{+}\left(\mathbb{H}^{2}\right)$, т.е.

\footnotetext{
${ }^{12}$ Если $\Gamma$ содержит элементы конечного порядка, то $M$ является поверхностью с особенностями или по терминологии Терстона орбифолдом. Пример: $\Gamma=P S L(2, \mathbb{Z})$.
} 
поверхности имеют одинаковую ориентацию, то, отождествив $\mathrm{Iso}^{+}\left(\mathbb{H}^{2}\right)$ и $P S L(2, \mathbb{R})$, можно считать, что $\tilde{\theta}=\alpha \in P S L(2, \mathbb{R})$. Тогда изометрия поверхностей и ее дифференщиал задаются отображениями

$$
\theta: \Gamma_{1} z \rightarrow \alpha \Gamma_{1} z=\Gamma_{2} \alpha z, z \in \mathbb{H}^{2}, \quad d \theta: \Gamma_{1} g \rightarrow \alpha \Gamma_{1} g=\Gamma_{2} \alpha g, g \in P S L(2, \mathbb{R})
$$

Если же их ориентации противоположны, то $\tilde{\theta}=\alpha s$, где $\alpha \in \mathrm{Iso}^{+}\left(\mathbb{H}^{2}\right)$ и $s$-отражение. В этом случае изометрия поверхностей и ее дифференциал есть

$$
\theta: \Gamma_{1} z \rightarrow \alpha \Sigma\left(\Gamma_{1}\right) s(z)=\Gamma_{2} \alpha s(z), \quad d \theta: \Gamma_{1} g \rightarrow \alpha \Sigma\left(\Gamma_{1}\right) \Sigma(g)=\Gamma_{2} \alpha \Sigma(g),
$$

где $\Sigma(g)=\sigma g \sigma^{-1}, \sigma=\left(\begin{array}{rr}1 & 0 \\ 0 & -1\end{array}\right), g \in P S L(2, \mathbb{R})$.

Таким образом, поверхности $M_{1}$ и $M_{2}$ изометричны тогда и только тогда, когда $k\left(M_{1}\right)=k\left(M_{2}\right)$ и подгруппы $\Gamma_{1}=\pi_{1}\left(M_{1}\right)$ и $\Gamma_{2}=\pi_{1}\left(M_{2}\right)$ внутренне сопряжены в $\operatorname{Iso}\left(\mathbb{H}^{2}\right)$.

Известно, что изоморфизм $\pi_{1}\left(M_{1}\right)$ и $\pi_{1}\left(M_{2}\right)$ означает гомеоморфность $M_{1}$ и $M_{2}$, но не их изометричность (даже если $k\left(M_{1}\right)=k\left(M_{2}\right)$ ). Это объясняется тем фактом, что две дискретные подгруппы (и даже решетки) $\Gamma_{1}$ и $\Gamma_{2}$ в $G=S L(2, \mathbb{R})$ могут быть изоморфны, но не сопряжены в $G$. Множество всех не сопряженных в $G$ вложений $\Gamma$ в $G$ наделяется естественной топологией и назьвается пространством Тейхмюллера $T(\Gamma)$ дискретной групшы Г (подробнее см. обзор [VS]).

\section{§. Метрическая жесткость орициклического потока}

1. Далее $M=\Gamma \backslash G / K$ - поверхность постоянной отрищательной кривизны, где $G=S L(2, \mathbb{R}), K=S O(2)$, и $S M=\Gamma \backslash G$ - ее единичное касательное расслоение. Предположим, что $\operatorname{vol}(M)<\infty$, т.е. $\Gamma$ - решетка в $G$ и $G$-инвариантная мера $\nu$ на $S M$ нормирована условием $\nu(S M)=1$. Сформулируем основные результаты о поведении геодезического и орициклического потоков на $S M$ (см. также [R84,95b]).

Хорошо известно, что геодезический поток $g_{t}$ эргодичен (Хопф [Но]) и перемешивает (Хедлунд [Не39]). Гельфанд и Фомин [GF] доказали, что он имеет счетнократньй лебеговский спектр. Кроме того, геодезический поток является $K$-потоком (Синай [Si60]). Отметим, что все эти результаты являются частными случаями результатов об аносовских потоках [An]. Наиболее сильным метрическим свойством геодезического потока является его бернуллиевость (Орнстейн и Вейсс $[\mathrm{OW}])$. На $S M$ имеется континуум взаимно сингулярных $g_{t}$-эргодических вероятностных мер (Синай [Si72], Боуэн [Во75]). Среди них стоит отметить меры длины, сосредоточенные на периодических орбитах; сингулярные к риманову объему, но в то же время строго положительные меры; а также меры с негладким носителем. Почти каждая орбита геодезического потока ввиду эргодичности плотна в $S M$. Кроме плотных и периодических орбит встречаются и орбиты, замыкание которых не является подмногообразием. Это относится, например, к локально замкнутьм, но не замкнутым орбитам, а также к орбитам, замыкание которых локально представляет собой произведение канторова множества на интервал (так устроено, например, морсовское минимальное множество [Mor]). Заметим, что пример локально замкнутых, но не замкнутых орбит дают так называемые 
гомоклинические точки, которые сушествуют у любых аносовских потоков. В окрестности же орбиты гомоклинической точки сушествует локально-несвязное компактное инвариантное множество, содержащее континуум различных минимальных множеств (и сосредоточенных на них эргодических мер) - см. [AS]. Если поверхность $M$ некомпактна, то имеются и орбиты, уходящие на бесконечность в обоих направлениях.

Орициклический поток $u_{t}$ (все сказанное в равной мере относится и к $h_{t}$ ) эргодичен (Хедлунд [Не39]) и также имеет счетнократный лебеговский спектр (Парасюк [Рa]). Согласно Маркусу [Mar78] он перемешивает с любой кратностью. В отличие от геодезического потока орищиклический поток имеет нулевую энтропию (Гуревич $[\mathrm{Gu}]$, в некомпактном случае см. Дани [Da77]). Если поверхность $M$ компактна, то орициклический поток строго эргодичен (Фюрстенберг [Fu72]) и минимален (Хедлунд [Не36]). В некомпактном случае за исключением гладкой меры $\nu$ на $S M$ любая $u_{t}$-эргодическая вероятностная мера является мерой длины, сосредоточенной на периодических орициклах (Дани и Смилли [DS]), а любая орбита либо плотна в $S M$, либо периодична (Хедлунд [Не36]).

Объясним вкратце, как расположены периодические орициклы в некомпактном многообразии $S M=\Gamma \backslash G$ (это хорошо видно на примере $\Gamma=P S L(2, \mathbb{Z})$ ). Ясно, что если орбита $x U_{\mathbb{R}} \subset \Gamma \backslash G$ периодична, то такова же и орбита точки $x A_{s}$ для любого $s \in \mathbb{R}$. Поэтому каждый периодический орицикл $x U_{\mathbb{R}}$ лежит в связном цилиндре $x A_{\mathbb{R}} U_{\mathbb{R}}$, состоящем целиком из периодических орициклов. Ясно, что два таких цилиндра либо не пересекаются, либо совпадают. Образуюшая $x A_{\mathbb{R}}$ такого шилиндра уходит на бесконечность в положительном направлении. В самом деле, если $x A_{t_{n}} \rightarrow y$ при $t_{n} \rightarrow+\infty$, то поскольку период орищикла точки $x A_{t_{n}}$ становится короче и короче при $t_{n} \rightarrow+\infty$, точка $y \in \Gamma \backslash G$ была бы неподвижной точкой орициклического потока (что невозможно ввиду дискретности $\Gamma$ ). Поэтому полуцилиндр $x A_{\mathbb{R}^{+}} U_{\mathbb{R}}$ является гладко вложенньм замкнутым подмногообразием в $\Gamma \backslash G$, которьй при уходе на бесконечность становится все уже и уже. Топологически поверхность $M$ получена из компактной поверхности, к которой приклеены несколько таких полуцилиндров (после вырезания из нее такого же количества дисков).

Что касается полуцилиндра $x A_{\mathbb{R}^{-}} U_{\mathbb{R}}$, то он является плотньм в $\Gamma \backslash G$. Этот факт также имеет весьма простое объяснение и имеет место для тех фуксовых групा $\Gamma \subset G$, для которых геодезический поток на $\Gamma \backslash G$ топологически перемешивает (т.е. не только для решеток). В самом деле, пусть $x \in \Gamma \backslash G$. Если поток $\left(\Gamma \backslash G, A_{\mathbb{R}}\right)$ топологически перемешивает, то для любой последовательности $\left\{t_{n} \rightarrow-\infty\right\}$ множество $\bigcup_{n} x O(1) A_{t_{n}}$ плотно в $\Gamma \backslash G$ для любой окрестности единицы $O(1) \subset G$. Поэтому для любой точки $y \in \Gamma \backslash G$ найдется последовательность $\left\{g_{n} \in O(1)\right\}$ такая, что $x g_{n} A_{t_{n}} \rightarrow y$. Но для достаточно малой окрестности $O(1) \subset G$ имеем $g_{n}=u_{n} a_{n} h_{n}$, где $u_{n} \in U_{\mathbb{R}}, a_{n} \in A_{\mathbb{R}}, h_{n} \in H_{\mathbb{R}}$, и поэтому $x g_{n} A_{t_{n}}=x u_{n} a_{n}^{\prime} h_{n}^{\prime}$, где $a_{n}^{\prime}=a_{n} A_{t_{n}} \in A_{\mathbb{R}}$, $h_{n}^{\prime}=A_{-t_{n}} h_{n} A_{t_{n}} \rightarrow 1$. Следовательно, $x u_{n} a_{n}^{\prime} \rightarrow y$ и поэтому $y \in \overline{x A_{\mathbb{R}^{-}} U_{\mathbb{R}}}$.

Заметим, что число таких непересекающихся цилиндров в $\Gamma \backslash G$ равно числу классов Г-сопряженности унипотентных подгрупп в Г. Хорошо известно, что это число конечно для любой решетки $\Gamma \subset G$ (так, для $\Gamma=P S L(2, \mathbb{Z})$ имеется всего один класс сопряженности).

Поскольку энтропия сдвига $g_{1}$ равна $\lambda=\sqrt{-k(M)}$ и геодезический поток является бернуллиевским, то по теореме Орнстейна [O] геодезические потоки на поверхностях 
$M_{1}$ и $M_{2}$ одинаковой постоянной кривизны метрически изоморфны. В этом смысле геодезический поток не является метрически жестким: метрический изоморфизм $g_{t}^{(1)}$ и $g_{t}^{(2)}$ не влечет за собой ни изометрию $M_{1}$ и $M_{2}$, ни даже их диффеоморфность. Заметим также, что геодезические потоки на компактных диффеоморфных поверхностях $M_{1}$ и $M_{2}$ топологически эквивалентны (Аносов $\left.[\mathrm{An}]\right)$. В самом деле, поверхность $M_{2}$ можно рассматривать как поверхность $M_{1}$ с измененной метрикой постоянной отрицательной кривизны. Если изменение метрики мало, то аносовский поток $g_{t}^{(2)}$ является малым $C^{1}$-возмущением потока $g_{t}^{(1)}$ и согласно теореме Аносова о структурной устойчивости эти потоки топологически эквивалентны. Теперь осталось заметить, что пространство метрик постоянной кривизны на компактной поверхности $M_{1}$ совпадает с пространством Тейхмюллера $T\left(\Gamma_{1}\right)$ групшы $\pi_{1}\left(M_{1}\right)=\Gamma_{1}$ и поэтому связно. Однако $M_{1}$ и $M_{2}$ могут быть не изометричны даже при их одинаковой кривизне (в случае, если $\Gamma_{1}$ и $\Gamma_{2}$ изоморфны, но не сопряжены в $\left.G\right)$. Следовательно, геодезический поток не является и топологически жестким.

Что касается орициклического потока $u_{t}$, то любые два потока $u_{t}^{(1)}$ и $u_{t}^{(2)}$ эквивалентны по Какутани, т.е. найдется измеримое отображение $\varphi: S M_{1} \rightarrow S M_{2}$ такое, что $\varphi$ переводит $u_{t}^{(1)}$-орбиты в $u_{t}^{(2)}$-орбиты с сохранением их ориентации (и является даже $C^{\infty}$-гладким вдоль $u_{t}^{(1)}$-орбит). Это следует из результата Ратнер [R78] о том, что орициклический поток эквивалентен по Какутани линейному иррациональному потоку на двумерном торе (заметим, что любые два иррациональных потока на двумерном торе эквивалентны по Какутани согласно Катку [K75]).

2. Рассмотрим теперь вопрос о том, когда два орициклических потока могут быть метрически изоморфны. Ясно, во-первых, что любая изометрия $\theta: M_{1} \rightarrow M_{2}$ двух одинаково ориентированных поверхностей индуцирует изоморфизм $d \theta$ потоков $g_{t}^{(1)}$, $u_{t}^{(1)}, h_{t}^{(1)}$ и $g_{t}^{(2)}, u_{t}^{(2)}, h_{t}^{(2)}$ соответственно. В самом деле, отображение $d \theta\left(\Gamma_{1} g\right)=\Gamma_{2} \alpha g$, где $\tilde{\theta}=f_{\alpha} \in \operatorname{Iso}^{+}\left(\mathbb{H}^{2}\right), \alpha \in G$ и $\alpha \Gamma_{1} \alpha^{-1}=\Gamma_{2}$, обладает свойством $d \theta\left(\Gamma_{1} g h\right)=$ $d \theta\left(\Gamma_{1} g\right) h$ для любых $g, h \in G$. Во-вторых, можно взять композицию дифференциала изометрии $d \theta$ и сдвига на постоянный орицикл $u_{s}^{(2)}$. Удивительно, что по доказанной в начале 80-х годов Мариной Ратнер теореме о метрической жесткости орищиклического потока любой метрический изоморфизм $u_{t}^{(1)}$ и $u_{t}^{(2)}$ происходит из такой композиции!

TeOpema 2.1 ([R82a]). Пусть $G=S L(2, \mathbb{R})$ u $\Gamma_{1}, \Gamma_{2}-$ две решетки в $G$. Предположим, что $\varphi:\left(\Gamma_{1} \backslash G, U_{t}, \nu_{1}\right) \rightarrow\left(\Gamma_{2} \backslash G, U_{t}, \nu_{2}\right)$ - метрическая сопряженность орициклических потоков. Тогда найдутся әлемент $\alpha \in G$ и число $s \in \mathbb{R}$ такие, что $\alpha \Gamma_{1} \alpha^{-1} \subset \Gamma_{2}$ u $\varphi\left(\Gamma_{1} g\right)=\Gamma_{2} \alpha g U_{s}$ почти всюду.

С ЛЕДСТВИЕ 2.2 ([R82a]). Пусть $M_{1}=\Gamma_{1} \backslash G / K$ u $M_{2}=\Gamma_{2} \backslash G / K-$ две ориентированных поверхности конечной площади с равными отрицательными кривизнами. Предположим, что $\varphi:\left(S M_{1}, \nu_{1}\right) \rightarrow\left(S M_{2}, \nu_{2}\right)$ - метрический изоморфизм орициклических потоков. Тогда $\varphi=u_{s}^{(2)} \circ d \theta$, ге $u_{s}^{(2)}-$ постоянный сдвиг вдоль орицикла и $d \theta$ - дифференииал некоторой изометрии $\theta: M_{1} \rightarrow M_{2}$, сохраняющей ориентацию.

СлЕДСТВИЕ 2.3 ([R83]). Предположим, что $M=\Gamma \backslash G / K$ - ориентированная поверхность постоянной отрицательной кривизнь конечной площади и 
$\varphi:(S M, \nu) \rightarrow(S M, \nu)$ - отображсение, устанавливающее изоморфизм $u_{t}$ $u u_{-t}$ для некоторого $t>0$. Тогда $\beta \Gamma \beta^{-1}=\Gamma$ для некоторого $\beta \in \operatorname{Iso}\left(\mathbb{H}^{2}\right)$, $\operatorname{det}(\beta)=-1$.

Для доказательства заметим, что отображение $\Sigma(\Gamma g)=\Sigma(\Gamma) \Sigma(g)$ устанавливает изоморфизм $u_{t}$ и $u_{-t}$, и поэтому $\Sigma$ о $\varphi$ задает изоморфизм $u_{t}$ с самим собой. Следовательно, $(\sigma \alpha) \Gamma(\sigma \alpha)^{-1}=\Gamma$ при некотором $\alpha \in G$.

Итак, орициклический поток изоморфен своему обратному лишш при наличии изометрии поверхности $M$, меняющей ориентацию. Напротив, геодезический поток всегда изоморфен своему обратному (достаточно взять диффеоморфизм $S M$, неподвижньй на $M$ и являюшийся инверсией на каждом касательном пространстве).

ЗАмечАниЕ. Одновременно с теоремой Ратнер о метрической жесткости Маркуcoм [Mar83] была доказана топологическая жесткость орициклического потока. Скажем, что метрика (возможно, переменной) отрицательной кривизны на поверхности $M$ нормализована, если длина кратчайшей периодической геодезической равна 1. Тогда орициклические потоки на двух компактных поверхностях отрицательной кривизны с нормализованными метриками топологически эквивалентны $\Longleftrightarrow$ соответствующие геодезические потоки топологически изоморфны [Mar83]. На самом деле топологический изоморфизм геодезических потоков влечет за собой изометричность поверхностей (см. §3).

\section{§3. Геометрические обобщения теоремы Ратнер о метрической жесткости орициклического потока}

Теорему о метрической жесткости можно обобщать в различных направлениях. Так, можно отказаться от постоянности кривизны поверхностей, повысить размерность (т.е. рассматривать многообразия отрицательной кривизны вместо поверхностей) и, наконец, отказаться от конечности объема этих многообразий.

1. Фельдман и Орнстейн [FO] доказали аналог теоремы жесткости для компактных поверхностей переменной отрицательной кривизны. Напомним, что если $M-$ гладкая компактная риманова поверхность отрицательной кривизны, то геодезический поток $g_{t}$ на $S M$ является аносовским и обладает гладкой инвариантной мерой. Сжимаюшееся и расширяюшееся слоения $\mathscr{U}$ и $\mathscr{H}$ на $S M$ для $g_{t}$ могут быть непрерьвно параметризованы различньми способами (гладкой параметризации, вообще говоря, не сушествует). Нам будут важны два способа параметризации. Первый (классический) состоит в том, что кусок траектории орицикла от любой точки $x \in S M$ за время $t$ проектируется на поверхность $M$ в кривую длины $t$. Эта параметризация назьвается параметризацией длиной дуги. Другой способ параметризации был предложен Маргулисом (см. [Ma70] и [Mar75]). Он доказал существование равномерных параметризаций $u_{t}$ и $h_{t}$, для которых независимо от точки $x \in S M$ выполняются соотношения

$$
g_{t} \circ u_{s}(x)=u_{\lambda^{-t} s} \circ g_{t}(x), \quad g_{t} \circ h_{s}(x)=h_{\lambda^{t} s} \circ g_{t}(x),
$$

где $\lambda>0$-некоторая константа (на самом деле $\lambda=e^{h\left(g_{1}\right)}$, где $h\left(g_{1}\right)$ - топологическая энтропия геодезического сдвига $\left.g_{1}\right)$. Заметим, что для случая постоянной кривизны с точностью до постоянной перенормировки времени оба этих способа совпадают с параметризацией однопараметрических подгрупп $U_{t}, H_{t} \subset G$. 
Маркус [Mar75] доказал, что оба этих способа параметризации задают строго эргодические перемешивающие потоки. Единственные эргодические инвариантные меры в обоих способах за исключением случая постоянной кривизны сингулярны друг к другу и к гладкой мере объема.

Далее будем считать, что $u_{t}$ и $h_{t}$ обозначают равномерные параметризации слоений $\mathscr{U}$ и $\mathscr{H}$. В этом случае единственной эргодической мерой для $u_{t}$ и $h_{t}$ является мера $\mu$ максимальной энтропии потока $g_{t}$. Заметим, что эта мера строго положительна, однако для метрики непостоянной кривизны не является гладкой. Риманову метрику на $M$ нормируем так, что $h\left(g_{1}\right)=1$, т.е. коэффициент растяжения $\lambda$ равен $e$.

При таких предположениях Фельдман и Орнстейн доказали, что метрический изоморфизм двух орициклических потоков на компактных поверхностях является композицией $C^{1}$-гладкого изоморфизма геодезических потоков и сдвига вдоль орицикла. Идея доказательства состоит в применении свойства Ратнер (см. §6) для орициклических потоков $u_{t}^{(1)}$ и $u_{t}^{(2)}$, чтобы доказать сопряженность $g_{t}^{(1)}$ и $g_{t}^{(2)}$ некоторым отображением $\psi$. Тот факт, что $\psi$ сопрягает $h_{t}^{(1)}$ и $h_{c t}^{(2)}$ для некоторого $c \in \mathbb{R}$ (и, таким образом, является гомеоморфизмом), требует отдельного доказательства (отличного от случая постоянной кривизны). Далее Фельдман и Орнстейн доказьвают, что гомеоморфизм $M_{1}$ и $M_{2}$, сопрягаюший $g_{t}^{(1)}$ и $g_{t}^{(2)}$, имеет класс гладкости $C^{1}$.

Если одна из поверхностей имеет постоянную кривизну, то по Катку [K82] $C^{1}$-гладкий изоморфизм $\psi: S M_{1} \rightarrow S M_{2}$ геодезических потоков есть дифференщиал некоторой изометрии $\theta: M_{1} \rightarrow M_{2}$. В общем случае это было независимо доказано Оталом $[\mathrm{Ot}]$ и Кроуком [Cr]. Таким образом, имеет место

Teорема 3.1 ([FO], [Ot], [Cr]). Пусть $M_{i}, i=1,2,-$ компактные поверхности нормированной отрицательной кривизны с мерами $\mu_{i}$ максимальной энтропии геодезических потоков $g_{t}^{(i)}$ на $S M_{i}$, и $u_{t}^{(i)}$ - равномерно параметризованные оричиклические потоки на $S M_{i}$. Предположим, что $\varphi:\left(S M_{1}, \mu_{1}\right) \rightarrow\left(S M_{2}, \mu_{2}\right)-$ метрический изоморфизм $u_{t}^{(1)} u u_{t}^{(2)}$. Тогда $\varphi=u_{s}^{(2)} \circ d \theta$, где $u_{s}^{(2)}-$ постоянный сдвиг вдоль орицикла и $\theta: M_{1} \rightarrow M_{2}$ - изометрия поверхностей.

Что касается первого способа параметризации слоения (длиной дуги), то для него подобные свойства жесткости не доказаны, хотя, как мы сейчас увидим, именно этот способ позволяет формулировать разумные гипотезы в многомерном случае.

2. Напомним, что $n$-мерное многообразие $M$ постоянной кривизны -1 является пространством двойных классов смежности $M=\Gamma \backslash G / K$, где $\Gamma$ - дискретная подгруппа в связной компоненте $G$ групшы Iso $\left(\mathbb{H}^{n}\right)$ изометрий $n$-мерного пространства Лобачевского $\mathbb{H}^{n}$ и $K$ - максимальная компактная подгруппа в $G$ (для гладкости $M$ требуется, чтобы пересечение $g \Gamma g^{-1} \cap K$ не зависело от $g \in G$ ). Само пространство $\mathbb{H}^{n}$ удобно представлять как один из двух связных кусков псевдосферы $\Sigma$ в $\mathbb{R}^{n+1}$, состоящей из точек $x=\left(x_{0}, x_{1}, \ldots, x_{n}\right)$, для которых $\langle x, x\rangle=1$, где $\langle\cdot, \cdot\rangle$ обозначает билинейную форму $\langle x, y\rangle=x_{0} y_{0}-x_{1} y_{1}-\cdots-x_{n} y_{n}$. Будем для определенности считать, что $\mathbb{H}^{n}=\Sigma^{+}$, где $\Sigma^{+}{ }_{-}$кусок $\Sigma$, для которого $x_{0}>0$.

Группа матрищ из $G L(n+1, \mathbb{R})$, сохраняющих форму $\langle\cdot, \cdot\rangle$, обозначается $O(1, n)$. Ее подгруппа индекса 2 , coxраняющая $\Sigma^{+}$, и является группой Iso( $\left.\mathbb{H}^{n}\right)$ изометрий пространства $\mathbb{H}^{n}$ с римановой метрикой, полученной ограничением $\langle\cdot, \cdot\rangle$ на $\Sigma^{+}$. Пусть $G$ - связная компонента групшы Iso $\left(\mathbb{H}^{n}\right)$ (заметим, что все элементы из $G$ сохраняют 
ориентацию $\left.\mathbb{H}^{n}\right)$ и

$$
K=\left(\begin{array}{cccc}
1 & 0 & \ldots & 0 \\
0 & & \\
\vdots & S O(n) \\
0 & &
\end{array}\right), \quad K_{1}=\left(\begin{array}{cccccc}
1 & 0 & 0 & \ldots & 0 \\
0 & 1 & 0 & \ldots & 0 \\
0 & 0 & & & \\
\vdots & \vdots & S O(n-1) &
\end{array}\right) .
$$

Тогда орбита точки $\hat{x}=(1,0, \ldots, 0)$ при действии $G$ на $\mathbb{R}^{n+1}$ совпадает с $\mathbb{H}^{n}=\Sigma^{+}$, причем $\mathbb{H}^{n}=G / K$, где $K$ - стабилизатор точки $\hat{x}$, причем $K \simeq S O(n)$ является максимальной компактной подгруппой в $G$. Таким образом, $M=\Gamma \backslash G / K$. Поскольку действие $K$ на единичном касательном пространстве $S_{\hat{x}} \mathbb{H}^{n}$ индуцирует транзитивную групшу поворотов $S O(n)$ сферы $S^{n-1}$ со стабилизатором $S O(n-1)$, то $S \mathbb{H}^{n}=G / K_{1}$ и $S M=\Gamma \backslash G / K_{1}$.

Геодезический поток $g_{t}$ на $S M$ индуцируется правым действием подгруппы

$$
A=\left\{A_{t}\right\}=\left(\begin{array}{ccccc}
\cosh (t) & \sinh (t) & 0 & \cdot & 0 \\
\sinh (t) & \cosh (t) & 0 & \cdot & 0 \\
0 & 0 & 1 & & \\
\cdot & \cdot & & \cdot & \\
0 & 0 & & & 1
\end{array}\right)
$$

на $S M=\Gamma \backslash G / K_{1}$. Поскольку $K_{1}$ и $A_{t}$ коммутируют, то действие $A_{t}$ на $S M$ определено корректно. $G$-инвариантная мера на $\Gamma \backslash G$ индуцирует $g_{t}$-инвариантную гладкую меру Лиувилля $\nu$ на $S M$. В свою очередь, $M$ имеет гладкую риманову меру объема vol. При этом $\operatorname{vol}(M)<\infty$ тогда и только тогда, когда $\nu(S M)<\infty$, т.е. когда $\Gamma-$ решетка в $G$.

Сжимающаяся орисферическая подгруппа $U \subset G$ для $A$ является $(n-1)$-мерной абелевой группой матрищ

$$
U=\left\{U_{a}\right\}=\left(\begin{array}{cccccc}
1+|a| / 2 & |a| / 2 & a_{2} & \cdot & \cdot & a_{n} \\
-|a| / 2 & 1-|a| / 2 & -a_{2} & \cdot & \cdot & -a_{n} \\
a_{2} & a_{2} & 1 & & \\
\cdot & \cdot & & 1 & \\
\cdot & \cdot & & & \cdot & \\
a_{n} & a_{n} & & & 1
\end{array}\right)
$$

где $a=\left(a_{2}, \ldots, a_{n}\right) \in \mathbb{R}^{n-1},|a|=a_{2}^{2}+\cdots+a_{n}^{2}$. При этом $A_{-t} U_{a} A_{t}=U_{e^{-t}}{ }_{a}$ и поэтому все направления в $U$ сжимаются равномерно. Поскольку $K_{1}$ как централизатор $A$ обязан нормализовать $U$ и $K_{1} \cap g U g^{-1}=1$ для всех $g \in G$, листы $\Gamma g U K_{1} \subset \Gamma \backslash G / K_{1}$ задают гладкое $(n-1)$-мерное сжимающееся слоение $\mathscr{U}$ для $g_{t}$. Но так как действие $K_{1}$ на $U$ неприводимо (не имеет инвариантных подпространств), то $\mathscr{U}$ не имеет параметризации или хотя бы подслоений алгебраического происхождения.

Все сказанное относится и к $(n-1)$-мерному расширяющемуся слоению $\mathscr{H}$, образованному листами $\Gamma g H K_{1} \subset \Gamma \backslash G / K_{1}$ транспонированной подгрупшы $H=U^{T}$.

Известно, что если объем многообразия $M$ конечен, т.е. $\nu(S M)<\infty$, то геодезический поток $g_{t}$ на $S M$ эргодичен и является $K$-потоком, а орисферические слоения 
$\mathscr{U}, \mathscr{H}$ топологически транзитивны (это следует, например, из того, что $g_{t}$ является аносовским перемешивающим потоком; можно также воспользоваться критерием эргодичности С.1 Мура для накрьвающих однородных потоков $\left(\Gamma \backslash G, A_{t}\right)$ и $\left.(\Gamma \backslash G, U)\right)$.

В геометрической формулировке теоремы жесткости для случая $n=2$ речь идет об измеримой сопряженности орициклических потоков. Такое отображение можно иначе определить как измеримое отображение, сохраняющее параметризацию сжимающегося одномерного слоения $\mathscr{U}$ длиной дуги. В многомерном случае аналогом является такое отображение, переводящее слои $\mathscr{U}_{1}$ в слои $\mathscr{U}_{2}$, что ограничение на каждый слой $\mathscr{U}_{1}$ является изометрией (риманова метрика на $M$ индуцирует риманову метрику на всех $(n-1)$-мерных проекциях орисфер, что позволяет индуцировать риманову метрику на всех слоях $\mathscr{U})$. Этот способ формулировки и доказательство теоремы жесткости в многомерном случае были найдены Фламинио:

Tеорема $3.2([\mathrm{~F}])$. Пусть $M_{1}$ и $M_{2}-$ два п-мерных $(n>2)$ компактных многообразия постоянной кривизнь -1 и $\varphi: S M_{1} \rightarrow S M_{2}-$ сохраняющ,е меру Лиувилля обратимое отображсение, переводящее каждый слой $\mathscr{U}_{1}$ изометрично на слой $\mathscr{U}_{2}$. Тогда ч есть дифференциал некоторой изометрии $\theta: M_{1} \rightarrow M_{2}$.

Заметим, что при $n>2$ метрическая сопряженность не включает в себя постоянной трансляции вдоль $\mathscr{U}_{2}$.

3. Расслоение $F M$ ортонормированных реперов положительной ориентации над $M$ является главным $S O(n)$-расслоением и есть не что иное, как расслоение $F M=$ $\Gamma \backslash G \rightarrow \Gamma \backslash G / K=M$. Таким образом, поток ортонормированных реперов над $M$ является однородным потоком $\left(\Gamma \backslash G, A_{t}\right)$, который накрьвает геодезический поток. Орбиты подгруппы $U$ на $\Gamma \backslash G$ задают сжимаюшийся орисферический поток. Теорема Витте о метрической жесткости эргодических унипотентных потоков (о которой речь пойдет в $\S 5$ ) гарантирует, что из метрического изоморфизма орисферических потоков на $F M_{1}$ и $F M_{2}$ следует, в частности, изометричность многообразий $M_{1}$ и $M_{2}$. Естественно поэтому было обобшить результаты Фламинио и Витте на класс $S O(n)$-расслоений $E M$ над $M$, промежуточных между $S M$ и $F M$ (такое расслоение образовано накрытием $\Gamma \backslash G / E \rightarrow \Gamma \backslash G / K$, где $E \subset K_{1}$ - некоторая связная компактная подгруппа). Мера $\nu$ на $E M$ получается из $G$-инвариантной меры на $\Gamma \backslash G$. Подгруппа $A_{t}$ задает частично-гиперболический поток на $E M$, а листы $\Gamma g U E$ образуют для него сжимаюшееся слоение. Метрику на каждой орисфере в $E M$ снова можно получить из ограничения римановой метрики на образ орисферы при проекции $E M \rightarrow M$.

ТеОРема 3.3 ([Wi89]). Пусть $M_{1}, M_{2}$ - два многообразия постоянной кривизньи -1 конечного облема и $E M_{i}, i=1,2$, - расслоения над $M_{i}$, промежуточные между $S M_{i}$ и FM $M_{i}$. Тогда если $\varphi: E M_{1} \rightarrow E M_{2}$ - сохраняющее меру обратимое отображсние, переводящее каждый орисферический слой $\mathscr{U}_{1}$ изометрично на орисферический слой $\mathscr{U}_{2}$, то многообразия $M_{1}$ и $M_{2}$ изометричньл.

Жесткость орисферических слоений на многообразиях переменной отрицательной кривизны до сих пор не установлена. Как уже отмечалось, для этого вначале нужно доказать жесткость орициклического потока на поверхности переменной кривизны, параметризованного длиной дуги. 
4. Фламинио и Спатциер [FS] показали, как теорему о метрической жесткости орисферических слоений можно обобщать для многообразий постоянной отрицательной кривизны бесконечного объема. Строение таких многообразий до сих пор мало изучено ввиду сложности устройства дискретных групп $\Gamma \subset G=O(1, n)_{0}=\operatorname{Iso}\left(\mathbb{H}^{n}\right)_{0}$ движения пространства Лобачевского $\mathbb{H}^{n}$ (обычно предполагают, что $Г$ не содержит элементов конечного порядка). Есть продвижения, однако, в изучении обширного класса так называемых геометрически-конечных дискретных подгрупп $\Gamma \subset G$ (это - группы, которые допускают фундаментальную область в $\mathbb{H}^{n}$ в виде гиперболически вьпуклого многогранника с конечным числом граней). Такая группа Г является конечно-порожденной, однако при $n>2$ не каждая конечно-порожденная дискретная группа движений $\mathbb{H}^{n}$ является геометрически-конечной (впрочем, если $\Gamma \backslash \mathbb{H}^{n}$ имеет конечньй объем, то Г геометрически-конечна). Замечательно, что для геометрически-конечных групш Г многообразие $S M=\Gamma \backslash S \mathbb{H}^{n}$ несет на себе $g_{t}$-инвариантную эргодическую конечную меру, сосредоточенную на множестве $\Lambda$ неблуждающих точек геодезического потока. Такая мера единственна с точностью до нормировки и назьвается мерой Паттерсона-Сулливана (в случае конечного объема она совпадает с мерой Лиувилля на $S M$ ). Если множество $\Lambda$ компактно, то Г назьвается группой компактного типа. Любая такая група Г геометрически-конечна и не содержит квазиунипотентных элементов бесконечного порядка (следовательно, каждый орисферический слой на $S M=\Gamma \backslash G / K_{1}$ односвязен и диффеоморфен $\mathbb{R}^{n-1}$ ). В [FS] показано, в частности, что если $\operatorname{dim} M_{1}=\operatorname{dim} M_{2}$, группа $\Gamma_{1}$ имеет компактньй тип и плотна по Зарисскому в $G$, то сохраняюшее меру Паттерсона-Сулливана отображение $\varphi: S M_{1} \rightarrow S M_{2}$, изометрично переводящее пересечения с $\Lambda_{1}$ сжимаюшихся орисферических слоев на $S M_{1}$ в сжимаюшиеся орисферические слои на $S M_{2}$, влечет сушествование риманова накрытия $\theta: M_{1} \rightarrow M_{2}$.

На взгляд автора, было бы интересно обобшить теорему о метрической жесткости орисферических слоений относительно гладкой бесконечной меры Лиувилля на $S M$. Например, естественно предположить, что эргодический относительно бесконечной меры Лиувилля орициклический поток на поверхности $M$ постоянной отрицательной кривизны обладает метрической жесткостью, как и в случае $\operatorname{vol}(M)<\infty$. Отметим, однако, что эргодичность орициклического потока установлена только в случае конечного объема $S M=\Gamma \backslash P S L(2, \mathbb{R})$, хотя известны фуксовы групшы $\Gamma$, для которых объем $S M$ бесконечен, а орициклический поток консервативен и топологически транзитивен (см. обзор [St95b]).

\section{§4. Факторы и джойнинги орициклических потоков}

Следуя $[\mathrm{R} 84,95 \mathrm{~b}]$, рассмотрим орициклический поток $u_{t}=\left(\Gamma \backslash G, U_{t}\right)$ на пространстве с конечной $G$-инвариантной мерой $\nu$. Если дискретная подгруппа $\Gamma^{\prime} \subset G$ содержит $\Gamma$, то орициклический поток $u_{t}^{\prime}=\left(\Gamma^{\prime} \backslash G, U_{t}\right)$ является фактором потока $u_{t}$. Как доказала Ратнер, каждый факторпоток для $u_{t}$ получается из указанной алгебраической конструкции:

TeOpema 4.1 ([R82b]). Пусть $S_{t}$ есть факторпоток для орициклического потока $u_{t}=\left(\Gamma \backslash G, U_{t}\right)$, где $\Gamma$ - решетка в $G$. Тогда найдется решетка $\Gamma^{\prime} \subset G$ такая, что $\Gamma \subset \Gamma^{\prime}$ и поток $S_{t}$ изоморфен потоку $u_{t}^{\prime}=\left(\Gamma^{\prime} \backslash G, U_{t}\right)$. 
Так как число решеток $\Gamma^{\prime} \subset G$, содержаших решетку $\Gamma \subset G$, конечно, то и число неизоморфных факторов для орициклического потока $u_{t}$ конечно. В частности, если решетка $\Gamma$ максимальна, то $u_{t}$ вообще не имеет нетривиальных факторов. В этом проявляется еще одно отличие от геодезического потока. В самом деле, поток $S_{t}$ является фактором бернуллиевского потока $T_{t}$ тогда и только тогда, когда $S_{t}$ также является бернуллиевским, а энтропия $e\left(S_{t}\right)$ не превьшает энтропии $e\left(T_{t}\right)$. Следовательно, число неизоморфных факторов геодезического потока несчетно.

ЗАмечАниЕ. В [R82b] высказана гипотеза о том, что теорема о факторах может быть обобщена на равномерно параметризованный орициклический поток на компактной поверхности переменной отрицательной кривизны.

Теорема о факторах, как и теорема о жесткости, легко вьводится из классификации эргодических джойнингов орициклических потоков. Напомним, что джойнингом двух орициклических потоков $u_{t}^{(1)}=\left(\Gamma_{1} \backslash G, U_{t}, \nu_{1}\right)$ и $u_{t}^{(2)}=\left(\Gamma_{2} \backslash G, U_{t}, \nu_{2}\right)$ является вероятностная $U_{t} \times U_{t}$-инвариантная мера на $\left(\Gamma_{1} \backslash G\right) \times\left(\Gamma_{2} \backslash G\right)$, которая проектируется в $G$-инвариантные меры $\nu_{1}$ и $\nu_{2}$. Приведем вначале алгебраическую конструкцию эргодических джойнингов для $u_{t}^{(1)}$ и $u_{t}^{(2)}$.

Предположим, что элемент $c \in G$ таков, что $\Gamma=\Gamma_{1} \cap c^{-1} \Gamma_{2} c$ является решеткой в $G$. Положим $\Omega_{c}=\left\{\left(\Gamma_{1} g, \Gamma_{2} c g\right), g \in G\right\}$. Тогда множество $\Omega_{c}$ инвариантно относительно диагональной подгруппы $\Delta_{G}=\{(g, g), g \in G\}$, причем $\Delta_{G}$ действует на $\Omega_{c}$ транзитивно со стационарной подгрупой $\Delta_{\Gamma} \cdot \Delta_{G}$-инвариантная вероятностная мера $\nu_{0}$ на $\Omega_{c}=\Delta_{\Gamma} \backslash \Delta_{G}$ является $U_{t} \times U_{t}$-эргодическим джойнингом потоков $u_{t}^{(1)}$ и $u_{t}^{(2)}$. Более обшая конструкция возникает как сдвиг множества $\Omega_{c}$ и меры $\nu_{0}$ на любой элемент из $G \times G$, коммутируюший с подгруппой $\Delta_{U}=\left\{U_{t} \times U_{t}, t \in \mathbb{R}\right\}$. Ясно, что каждый такой сдвиг можно записать как $\Omega_{c^{\prime}, s}=\left\{\left(\Gamma_{1} g, \Gamma_{2} c^{\prime} g U_{s}\right), g \in G\right\}$ для некоторого $s \in \mathbb{R}$ и $c^{\prime} \in c \mathbb{Z}_{2}$. Следуюший результат Ратнер показьвает, что все нетривиальные эргодические джойнинги $u_{t}^{(1)}$ и $u_{t}^{(2)}$ возникают подобным образом.

TеОрема $4.2([\mathrm{R} 83])$. Пусть мера т на $\left(\Gamma_{1} \backslash G\right) \times\left(\Gamma_{2} \backslash G\right)$ является эргодическим джсойнингом для $u_{t}^{(1)}=\left(\Gamma_{1} \backslash G, U_{t}, \nu_{1}\right)$ u $u_{t}^{(2)}=\left(\Gamma_{2} \backslash G, U_{t}, \nu_{2}\right)$. Тогда либо $m=$ $\nu_{1} \times \nu_{2}$, либо найдутся элемент $c \in G$ и число $s \in \mathbb{R}$ такие, что $\Gamma=\Gamma_{1} \cap c^{-1} \Gamma_{2} c$ является решеткой в $G, \nu\left(\Omega_{c, s}\right)=1$ и поток $\left(\left(\Gamma_{1} \backslash G\right) \times\left(\Gamma_{2} \backslash G\right), U_{t} \times U_{t}, m\right)$ изоморфен оричиклическому потоку $\left(\Gamma \backslash G, U_{t}, \nu_{0}\right)$.

СлЕДСТВИЕ 4.3 ([R83]). Число неизоморфных эргодических джсойнингов двух ориииклических потоков не более чем счетно. Если решетка $\Gamma_{1}$ равномерна или арифметична, а решетка $\Gamma_{2}$ таковой не является, то потоки $u_{t}^{(1)} u u_{t}^{(2)}$ дизбюнктныл.

Заметим, что число неизоморфных эргодических джойнингов любых двух бернуллиевских (в частности, геодезических) потоков несчетно.

Отметим еше одно интересное следствие теоремы 4.2. Если решетка $\Gamma_{1}$ равномерна и арифметична, а решетка $\Gamma_{2}$ равномерна и неарифметична, то однопараметрический унипотентньй поток $\left(\left(\Gamma_{1} \backslash G\right) \times\left(\Gamma_{2} \backslash G\right), U_{t} \times U_{t}\right)$ строго эргодичен. Это следует из классификации джойнингов и строгой эргодичности орициклического потока на $\Gamma_{1} \backslash G$ и $\Gamma_{2} \backslash G$. В этом примере интересно то, что однопараметрическая подгруппа $\Delta_{U}=\left\{U_{t} \times U_{t}, t \in \mathbb{R}\right\}$ неорисферична (орисферической является двумерная подгруппа $\left.U_{\mathbb{R}} \times U_{\mathbb{R}}\right)$. До полученной М. Ратнер теоремы 4.2 единственными примерами строго 
эргодических потоков на компактных однородных пространствах полупростой групшы Ли были орисферические потоки (см. [Во76], [V] или [EP]).

Глазнер и Вейсс $[\mathrm{GW}]$ построили пример двух максимальных равномерных ариффметических решеток $\Gamma_{1}$ и $\Gamma_{2}$ таких, что $\Gamma_{1}$ и $\Gamma_{2}$ не сопряжены и $\Gamma_{1} \cap \Gamma_{2}$ имеет конечньй индекс в $\Gamma_{1}$ и $\Gamma_{2}$. Таким образом, минимальные орициклические потоки $u_{t}^{(1)}$ и $u_{t}^{(2)}$ не являются дизъюнктными. Это дает отрицательный ответ на вопрос Фюрстенберга, являются ли минимальные потоки без обших факторов обязательно дизъюнктными?

Рассмотрим теперь вопрос о числе неизоморфных эргодических автоджойнингов орищиклического потока $u_{t}=\left(\Gamma \backslash G, U_{t}, \nu\right)$. Для решетки $\Gamma \subset G$ обозначим через $\bar{\Gamma}$ подгрупу всех элементов $c \in G$ таких, что пересечение $\Gamma \cap c^{-1} \Gamma c$ является решеткой в $G$. Согласно Маргулису (см. [Ma90a]) подгрупа $\bar{\Gamma}$ плотна в $G$ в том и только том случае, когда решетка $\Gamma$ арифметична. В противном случае подгруппа $\bar{\Gamma}$ дискретна и, таким образом, содержит Г в качестве подгруппы конечного индекса.

СлЕДСТвИЕ 4.4 ([R83]). Если решетка $\Gamma \subset G$ арифметична, то орициклический поток $u_{t}=\left(\Gamma \backslash G, U_{t}, \nu\right)$ имеет счетное число неизоморфных әргодических автоджойнингов. Если решетка $\Gamma \subset G$ неарифметична, то и имеет конечное число неизоморфных әргодических автоджойнингов, а если Г вдобавок максимальна, то и не имеет нетривиальных әргодических автоджойнингов.

Покажем теперь, следуя [R83], как теорема о жесткости орициклических потоков вьводится из классификации эргодических джойнингов. Пусть $\varphi:\left(\Gamma_{1} \backslash G, U_{t}, \nu_{1}\right) \rightarrow$ $\left(\Gamma_{2} \backslash G, U_{t}, \nu_{2}\right)$ - метрическая сопряженность орищиклических потоков. Рассмотрим график

$$
\Omega=\left\{(x, \varphi(x)), x \in \Gamma_{1} \backslash G\right\} \subset\left(\Gamma_{1} \backslash G\right) \times\left(\Gamma_{2} \backslash G\right)
$$

вместе с вероятностной мерой $m$, заданной по правилу

$$
m(A \times B)=\int_{A} m_{\varphi(x)}(B) d \nu_{1}(x),
$$

где мера $m_{y}, y \in \Gamma_{2} \backslash G$, сосредоточена в точке $y$. Тогда множество $\Omega$ и мера $m$ являются $U_{t} \times U_{t}$-инвариантньми, причем $m$ является джойнингом двух наших потоков. Так как проекция $p_{1}: \Omega \rightarrow \Gamma_{1} \backslash G$ взаимно однозначна, то мера $m$ эргодична и из классификации эргодических джойнингов вытекает существование элемента $c \in G$ и числа $s \in \mathbb{R}$ таких, что пересечение $\Gamma=\Gamma_{1} \cap c^{-1} \Gamma_{2} c$ является подгруппой конечного индекса в $\Gamma_{1}$ и $c^{-1} \Gamma_{2} c$, причем $\varphi\left(\Gamma_{1} g\right)=\Gamma_{2} c g U_{s}$. Наконец, из взаимной однозначности $p_{1}$ вытекает, что $\Gamma=\Gamma_{1}$, и поэтому $\Gamma_{1} \subset c^{-1} \Gamma_{2} c$.

\section{§5. Жесткость, факторы и джойнинги унипотентных потоков}

1. В этом параграфе мы покажем, как теоремы Ратнер о жесткости, факторах и джойнингах орициклических потоков обобшаются на класс эргодических унипотентных потоков на однородных пространствах конечного объема. Дадим вначале необходимые определения.

ОПРЕДЕЛЕНИЕ 5.1. Пусть $\Gamma \backslash G, \Lambda \backslash H$ - два однородных пространства с мерами $\nu_{1}$ и $\nu_{2}$. Тогда сохраняющее меру отображение $\varphi:\left(\Gamma \backslash G, \nu_{1}\right) \rightarrow\left(\Lambda \backslash H, \nu_{2}\right)$ назьвается аффинным для $g \in G$, если найдется элемент $\tilde{g} \in H$ такой, что $\varphi\left(\Gamma g^{\prime} g\right)=\varphi\left(\Gamma g^{\prime}\right) \tilde{g}$ для $\nu_{1}$-почти всех $g^{\prime} \in G$. 
ОПреДЕЛЕНИЕ 5.2. Сохраняющее меру отображение $\varphi:\left(\Gamma \backslash G, \nu_{1}\right) \rightarrow\left(\Lambda \backslash H, \nu_{2}\right)$ называется $G$-афффинным, если оно афффинно для каждого $g \in G$.

Явньй вид $G$-афффинного отображения проясняет

ПРЕДЛОЖЕНИЕ 5.3 (см. [Wi85]). Пусть Г и $\Lambda$ - решетки в группах Ли $G$ и $H$ соответственно и $\varphi: \Gamma \backslash G \rightarrow \Lambda \backslash H$ - сохраняющее меру оббема отображение. Тогда при условии несократимости решетки $\Lambda \subset H$ следующие условия әквивалентны:

1) $\varphi$ является $G$-аффинным,

2) график $\operatorname{Graph}(\varphi)=\{(x, \varphi(x)), x \in \Gamma \backslash G\}$ является $\bmod 0$ однороднымм подпространством в $\Gamma \backslash G \times \Lambda \backslash H$,

3) найдутся әлемент $c \in H$ и эпиморфизм $\sigma: G \rightarrow H$ такие, что $\sigma(\Gamma) \subset \Lambda$ и $\varphi(\Gamma g)=\Lambda \sigma(g)$ с почти всюду на $\Gamma \backslash G$.

ДокАЗАТЕЛЬСтво. $1 \Rightarrow 2$. По Зиммеру $[\mathrm{Z}]$ отображение $\varphi$ можно так скорректировать на множестве меры 0 в $\Gamma \backslash G$, что равенство $\varphi\left(\Gamma g^{\prime} g\right)=\varphi\left(\Gamma g^{\prime}\right) \tilde{g}$ будет вьполняться для всех $g, g^{\prime} \in G$. Поскольку $\varphi\left(\Gamma g^{\prime}\right)=\varphi\left(\Gamma g^{\prime} 1\right)=\varphi\left(\Gamma g^{\prime}\right) \tilde{1}$ и отображение $\varphi$ является сюръективньм, то элемент 1 лежит в пересечении $\Lambda$ с центром $Z(H)$. Но так как решетка $\Lambda$ несократима, то $\tilde{1}=1$. Аналогично доказывается, что $\widetilde{g g^{\prime}}=\tilde{g} \tilde{g}^{\prime}$. Поэтому множество $F=\{(g, \tilde{g}), g \in G\}$ образует подгруппу в $G \times H$, которая транзитивно действует на $\operatorname{Graph}(\varphi)$.

$2 \Rightarrow 3$. Пусть $p: G \times H \rightarrow G$ и $p^{*}: \Gamma \backslash G \times \Lambda \backslash H \rightarrow \Gamma \backslash G$-естественные проекции и предположим, что $\operatorname{Graph}(\varphi)=(\Gamma \times \Lambda) F c$ для некоторого $c=\left(c_{1}, c_{2}\right) \in G \times H$ и подгрупш $F \subset G \times H$. По определению графика ограничение $p^{*}: \operatorname{Graph}(\varphi) \rightarrow \Gamma \backslash G$ является биекцией, и поэтому ограничение $p: F \rightarrow G$ является эпиморфизмом с ядром $F \cap H \subset \Lambda$. Но так как решетка $\Lambda$ несократима, то $p: F \rightarrow G$ является изоморфизмом. Обозначим через $\lambda: G \rightarrow F$ обратньй к нему изоморфизм и пусть $\sigma$ есть композиция $\lambda$ и проекции $q: G \times H \rightarrow H$. Пусть $(\Gamma g, \varphi(\Gamma g))=(\Gamma \times \Lambda) f\left(c_{1}, c_{2}\right)$ для некоторых $g \in G, f \in F$. Тогда $f=\left(g c_{1}^{-1}, \sigma\left(g c_{1}^{-1}\right)\right)$ и поэтому $\varphi(\Gamma g)=\Lambda \sigma(g) \sigma\left(c_{1}\right)^{-1} c_{2}$ для всех $g \in G$.

$3 \Rightarrow 1$. Положим $\tilde{g}=c^{-1} \sigma(g) c$. Тогда $\varphi\left(\Gamma g^{\prime} g\right)=\Lambda \sigma\left(g^{\prime} g\right) c=\Lambda \sigma\left(g^{\prime}\right) c c^{-1} \sigma(g) c=$ $\varphi\left(\Gamma g^{\prime}\right) \tilde{g}$, что и требовалось.

Итак, $G$-аффинное отображение является композицией однородного накрытия и постоянной трансляции. Под таким отображением можно понимать и отображение вида $\varphi(\Gamma g)=\Lambda c \alpha(g)$, где $\alpha: G \rightarrow H$ - такой эпиморфизм, что $\alpha(\Gamma) \subset c^{-1} \Lambda c$. В самом деле, достаточно положить $\alpha(g)=c^{-1} \sigma(g) c$ для всех $g \in G$.

Теперь мы можем дать алгебраическую формулировку теоремы 2.1 о жесткости орищиклических потоков.

Teорема 5.4. Пусть $G=S L(2, \mathbb{R})$ и $\Gamma_{1}, \Gamma_{2}-$ две решетки в $G$. Предположим, что сохраняющее меру отображсение $\varphi: \Gamma_{1} \backslash G \rightarrow \Gamma_{2} \backslash G$ является $U_{t}$-аффиннылм для некоторого $t>0$. Тогда оно является и $G$-аффинным.

Этот результат, как показал Витте, сохраняет силу в классе эргодических унипотентных действий. 


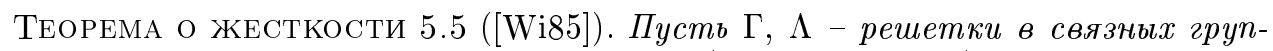
пах Ли $G, H$ соответственно и $\varphi:\left(\Gamma \backslash G, u_{\mathbb{Z}}^{(1)}\right) \rightarrow\left(\Lambda \backslash H, u_{\mathbb{Z}}^{(2)}\right)$ - метрическая сопряженность двух эргодических унипотентных каскадов. Тогда ч является $G$-аффинныц. В частности, если отображение $\varphi$ (почти всюду) конечнолистно, то группы $G$ и $H$ локально изоморфны. Если жсе отображение $\varphi$ обратимо (т.е. является метрическим изоморфизмом) и обе решетки Г и $\Lambda$ несократимы, mо $\varphi(\Gamma g)=\Lambda c \alpha(g)$ для некоторого әлемента $c \in G$ и изоморфизма $\alpha: G \rightarrow H$ mакого, ито $\alpha(\Gamma)=c^{-1} \Lambda c$.

Прямое доказательство этой теоремы весьма нетривиально. Оно потребовало от Витте формулировки и доказательства $R$-свойства (см. $\S 6$ для унипотентных транслящий на произвольных однородных пространствах. Однако теперь (после доказательства основной метрической теоремы Ратнер) она элементарно вьводится из классификации эргодических джойнингов унипотентных трансляций (или из самой метрической теоремы). Вьвод полностью аналогичен доказательству теоремы о жесткости орициклических потоков из $\S 4$.

2. Обратимся теперь к рассмотрению факторов эргодических унипотентных потоков. Алгебраичность факторов (как и теорема о жесткости) для эргодических нильпотоков была доказана еще Перри [Р71,73]. Этот результат и теорема Ратнер о факторах орициклического потока были обобщены Витте.

Пусть $\Gamma$ - решетка в группе Ли $G, U \subset G$ - унипотентная подгруппа и поток $(\Gamma \backslash G, U)$ эргодичен. Предположим, что $\Lambda \subset G$ есть замкнутая подгруппа в $G$, содержашая $\Gamma$. Тогда поток $(\Lambda \backslash G, U)$ является фактором исходного потока $(\Gamma \backslash G, U)$. Если $K \subset G$ является компактной подгруппой в $G$, коммутирующей с $U$, то действие $U$ на двойном классе смежности $\Lambda \backslash G / K$ также определяет факторпоток для $(\Gamma \backslash G, U)$. Наконец, в качестве $K$ можно взять любую замкнутую подгруппу в группе $\operatorname{Aff}(\Lambda \backslash G)$ всех $G$-аффинных отображений пространства $\Lambda \backslash G$, которая коммутирует с $U$ и для которой пространство орбит $\Lambda \backslash G / K$ является счетно отделимым (что эквивалентно локальной замкнутости почти всех $K$-орбит на $\Lambda \backslash G)$. Следуюшая теорема показывает, что так возникают все измеримые факторпотоки для эргодических унипотентных потоков.

TeOPEMa о ФакTOPAX 5.6 ([Wi94]). Пусть унипотентный поток $(\Gamma \backslash G, U)$ на пространстве конечного облема эргодичен. Тогда любой его измеримый факторпоток изоморфен потоку вида $(\Lambda \backslash G / K, U)$, где замкнутая подгруппа $\Lambda \subset G$ содержит $\Gamma$, а подгруппа $K \subset \operatorname{Aff}(\Lambda \backslash G)$ коммутирует с $U$ и определяет измеримое пространство $\Lambda \backslash G / K$.

Приведем вкратце доказательство этой теоремы. Пусть $\psi:(\Gamma \backslash G, \mu) \rightarrow(X, \nu)$ есть измеримая $U$-сопряженность. Рассмотрим

$$
\Lambda=\{\lambda \in G \mid \psi(\Gamma \lambda g)=\psi(\Gamma g), g \in G\} .
$$

Нетрудно доказать, что $\Lambda$ - замкнутая подгруппа в $G$ и поэтому пространство $\Lambda \backslash G$ имеет $G$-инвариантную вероятностную меру $\bar{\mu}$, а отображение $\psi$ определяет измеримую $U$-сопряженность $\bar{\psi}:(\Lambda \backslash G, \bar{\mu}) \rightarrow(X, \nu)$, а именно, $\bar{\psi}(\Lambda g)=\psi(\Gamma g), g \in G$. Пусть $K$ обозначает подгруппу всех элементов $\varphi \in \operatorname{Aff}(\Lambda \backslash G)$, которые коммутируют с $U$ 
и сохраняют слои отображения $\bar{\psi}$, т.е. $\bar{\psi}(\varphi(s))=\bar{\psi}(s)$ для почти всех $s \in \Lambda \backslash G$. Докажем, что пространство $X$ изоморфно $\Lambda \backslash G / K$.

Для этого рассмотрим множество

$$
M=\{(\Lambda s, \Lambda t) \in(\Lambda \backslash G) \times(\Lambda \backslash G) \mid \bar{\psi}(\Lambda s)=\bar{\psi}(\Lambda t)\}
$$

Достаточно доказать, что $K$ действует транзитивно на слоях $\bar{\psi}$, т.е. для любой пары $(\Lambda s, \Lambda t) \in M$ найдется $\varphi \in K$ такое, что $\varphi(\Lambda s)=\Lambda t$. Разложим меру $\bar{\mu}$ на вероятностные меры $\bar{\mu}_{x}$, сосредоточенные на слоях $\psi^{-1}(x), x \in X$. Это означает, что для любого борелевского множества $A \subset \Lambda \backslash G$ мы имеем $\bar{\mu}(A)=\int_{X} \bar{\mu}_{x}(A) d \nu(x)$. Теперь введем вероятностную меру $\rho$ на $(\Lambda \backslash G) \times(\Lambda \backslash G)$ по правилу

$$
\rho(A \times B)=\int_{X} \bar{\mu}_{x}(A) \bar{\mu}_{x}(B) d \nu(x) .
$$

Непосредственно проверяется, что мера $\rho$ сосредоточена на множестве $M$, причем $M$ и $\rho$ инвариантны относительно диагональной подгруппы $\Delta_{U}=\{(u, u), u \in U\}$, а мера $\rho$ является автоджойнингом меры $\bar{\mu}$. Из классификации джойнингов или метрической теоремы Ратнер вытекает, что эргодическая компонента меры $\rho$, содержашая точку $(\Lambda s, \Lambda t) \in M$, сосредоточена на замкнутой орбите $(\Lambda s, \Lambda t) H$ и является $H$-инвариантной для некоторой подгруппы $H \subset G \times G$ (причем $H$ проектируется эпиморфно на каждую из двух копий $G$ и содержит $\left.\Delta_{U}\right)$.

Докажем, что однородное пространство $(\Lambda s, \Lambda t) H$ является графиком некоторого аффинного отображения $\varphi: \Lambda \backslash G \rightarrow \Lambda \backslash G$. Положим $\varphi(\Lambda s g)=\Lambda t g^{\prime}$ для каждой пары $\left(g, g^{\prime}\right) \in H$. Корректность задания $\varphi$ вытекает из соотношения $H \cap\left(s^{-1} \Lambda s \times G\right) \subset$ $s^{-1} \Lambda s \times t^{-1} \Lambda t$. Проверим его, например, для $s=t=1 \in G$. Пусть $\lambda \in \Lambda$ и $(\lambda, h) \in H$. Так как $(\Lambda, \Lambda) H \subset M$, то для всех $\left(g, g^{\prime}\right) \in H$ мы имеем $\bar{\psi}\left(\Lambda h g^{\prime}\right)=\bar{\psi}(\Lambda \lambda g)=\bar{\psi}(\Gamma g)=$ $\bar{\psi}\left(\Gamma g^{\prime}\right)$ и поэтому $h \in \Lambda$. Аффинность отображения $\varphi$ и его коммутируемость с $U$ следуют из определения.

ЗАмЕчАНИЕ. Для орициклического потока (когда $G=S L(2, \mathbb{R})$ ) единственной подгруппой $K \subset \operatorname{Aff}(\Gamma \backslash G)$, имеющей “хорошее" пространство орбит $\Lambda \backslash G / K$ и коммутируюшей с действием потока, является подгруппа сдвигов вдоль центра $\mathbb{Z}_{2} \subset S L(2, \mathbb{R})$. В этом случае для $\Lambda^{\prime}=\mathbb{Z}_{2} \Lambda$ мы замечаем, что $\Lambda \backslash G / K=\Lambda^{\prime} \backslash G$, тем самым избавляясь от рассмотрения двойного класса смежности и получая теорему 4.1.

3. Теперь мы сформулируем и дадим упрощенньй вывод классификации эргодических джойнингов произвольных унипотентных потоков, используя основную метрическую теорему Ратнер.

ТЕОРЕМА О ДЖОЙНИНГАХ 5.7 ([R90а]). Пусть $G_{i}, i=1,2,-$ связные группь Ли с решетками $\Gamma_{i}$ и унипотентными подгруппами $u_{t}^{(i)}$ соответственно. Предположим, что мера $\mu$ на $\left(\Gamma_{1} \backslash G_{1}\right) \times\left(\Gamma_{2} \backslash G_{2}\right)$ является әргодическим джсойнигом для потоков $\left(\Gamma_{1} \backslash G_{1}, u_{t}^{(1)}, \nu_{1}\right) u\left(\Gamma_{2} \backslash G_{2}, u_{t}^{(2)}, \nu_{2}\right)$. Тогда найдутся замкнутая подгруппа $\Lambda \subset G_{1} \times G_{2}$ и элемент $c \in G_{2}$ такие, что

(1) подгруппьи $\Lambda_{i}=\Lambda \cap G_{i}$ нормальнье в $G_{i}$, 
(2) имеется непрерывный эпиморфизм $\alpha: G_{1} \rightarrow G_{2} / \Lambda_{2} c$ ядром $\Lambda_{1}$,

(3) пересечение $\alpha\left(\Gamma_{1}\right) \cap c^{-1} \Gamma_{2} c \Lambda_{2}$ является решеткой в $G_{2} / \Lambda_{2}$,

(4) мера $\mu$ является $\Lambda$-инвариантной мерой, сосредоточенной на замкнутой opбume $\left(\Gamma_{1}, \Gamma_{2} c\right) \Lambda$.

Если через $\bar{\alpha}(g) \subset G_{2}, g \in G_{1}$, обозначить полный прообраз әлемента $\alpha(g) \in$ $G_{2} / \Lambda_{2}$ в $G_{2}, m o \operatorname{supp} \mu=\left(\Gamma_{1}, \Gamma_{2} c\right) \Lambda=\left\{\left(\Gamma_{1} g, \Gamma_{2} c \bar{\alpha}(g)\right), g \in G_{1}\right\}$.

ДоКАЗАТЕЛЬСТвО. Пусть $p_{i}: G_{1} \times G_{2} \rightarrow G_{i}$ и $p_{i}^{*}:\left(\Gamma_{1} \backslash G_{1}\right) \times\left(\Gamma_{2} \backslash G_{2}\right) \rightarrow \Gamma_{i} \backslash G_{i}-$ естественные проекции. Из основной метрической теоремы Ратнер следует, что найдутся точка $x \in\left(\Gamma_{1} \backslash G_{1}\right) \times\left(\Gamma_{2} \backslash G_{2}\right)$ и замкну тая подгруппа $\Lambda \subset G_{1} \times G_{2}$ такие, что мера $\mu$ инвариантна относительно $\Lambda$ и сосредоточена на замкнутой орбите $x \Lambda$. Так как $\mu$ является джойнингом, то $p_{i}^{*}(\mu)=\nu_{i}$ и поэтому $p_{i}(\Lambda)=G_{i}, i=1,2$. Так как подгруппа $\Lambda_{i}=\Lambda \cap G_{i}$ нормальна в $\Lambda$ и $p_{i}(\Lambda)=G_{i}$, то $\Lambda_{i}$ нормальна в $G_{i}, i=1,2$.

Обозначим через $\bar{p}_{1}: \Lambda \rightarrow G_{1}$ эпиморфизм групп, полученный сужением $p_{1}$ на $\Lambda$. Для каждой пары $(g, \bar{g}) \in \Lambda$ положим $\alpha(g)=\bar{g} \Lambda_{2} \in p_{2} \bar{p}_{1}^{-1}(g) \Lambda_{2} \in G_{2} / \Lambda_{2}$. Тогда $\alpha: G_{1} \rightarrow G_{2} / \Lambda_{2}-$ корректно определенньй эпиморфизм групп с ядром $\Lambda_{1}$. Заметим также, что $\bar{\alpha}(g)=p_{2} \bar{p}_{1}^{-1}(g)$. Легко видеть, что $\left(H_{1}, H_{2}\right) \Lambda \cap G_{2}=H_{2} \bar{\alpha}\left(H_{1}\right)$ для любых подгрупш $H_{1} \subset G_{1}, H_{2} \subset G_{2}$.

Так как $p_{1}^{*}(x \Lambda)=\Gamma_{1} \backslash G_{1}$, то без ограничения общности можно считать, что $x=$ $\left(\Gamma_{1}, \Gamma_{2} c\right)$ для некоторого $c \in G_{2}$, и поэтому

$$
\left(\Gamma_{1}, \Gamma_{2} c\right) \Lambda=\left\{\left(\Gamma_{1} g, \Gamma_{2} c \bar{\alpha}(g)\right), g \in G_{1}\right\} .
$$

Поскольку орбита $\left(\Gamma_{1}, \Gamma_{2} c\right) \Lambda$ замкнута, произведение $\left(\Gamma_{1} \times c^{-1} \Gamma_{2} c\right) \Lambda \subset G_{1} \times G_{2}$ также замкнуто. Следовательно, замкнуты пересечение

$$
\left(\Gamma_{1} \times c^{-1} \Gamma_{2} c\right) \Lambda \cap G_{2}=c^{-1} \Gamma_{2} c \bar{\alpha}\left(\Gamma_{1}\right) \subset G_{2}
$$

и произведение $c^{-1} \Gamma_{2} c \alpha\left(\Gamma_{1}\right) \Lambda_{2} \subset G_{2} / \Lambda_{2}$. Теперь из счетности $\Gamma_{1}$ и $\Gamma_{2}$ вытекает, что подгруппы $\alpha\left(\Gamma_{1}\right)$ и $c^{-1} \Gamma_{2} c \Lambda_{2}$ дискретны в $G_{2} / \Lambda_{2}$ и потому являются там решетками. Наконец, слой расслоения однородных пространств конечного объема $p_{1}^{*}:\left(\Gamma_{1}, \Gamma_{2} c\right) \Lambda \rightarrow \Gamma_{1} \backslash G_{1}$ изоморфен однородному пространству $\bar{\alpha}\left(\Gamma_{1}\right) / \bar{\alpha}\left(\Gamma_{1}\right) \cap c^{-1} \Gamma_{2} c \Lambda_{2}$, которое, тем самым, также должно иметь конечный объем. Следовательно, пространство $\alpha\left(\Gamma_{1}\right) / \alpha\left(\Gamma_{1}\right) \cap c^{-1} \Gamma_{2} c \Lambda_{2}$ конечно, и теорема доказана.

Как следствие классификации джойнингов мы можем заключить, что каждый нетривиальный эргодический джойнинг двух унипотентных потоков на однородных пространствах простых групп Ли задает локальный изоморфизм групп. Отметим также, что данная общая классификация согласуется с классификацией 4.2 нетривиальных эргодических джойнингов орициклических потоков. Нужно лишь положить $\Lambda_{1}=\Lambda_{2}=1, \alpha(g)=U_{s}^{-1} g U_{s}, c^{\prime}=c U_{s}$. Тогда $\Omega=\left\{\left(\Gamma_{1} g, \Gamma_{2} c g U_{s}\right), g \in G\right\}=\left\{\left(\Gamma_{1} g, \Gamma_{2} c^{\prime} \alpha(g), g \in G\right)\right\}$.

\section{§ 6. Динамика орициклического потока}

Здесь мы докажем метрическую гипотезу для потока орициклов $(\Gamma \backslash S L(2, \mathbb{R}), U)$, не пользуясь орисферичностью подгруппы $U$. Это послужит демонстрацией доказательства Ратнер в общем случае. Изложение следует [R92], см. также [Gh]. 
Далее мы будем рассматривать группу Ли $G=S L(2, \mathbb{R})$, снабженную левоинвариантной метрикой $d_{G}$. Введем следуюшие обозначения для подгрупп: $U=\{u(s)$, $s \in \mathbb{R}\}, A=\{a(\tau), \tau \in \mathbb{R}\}, H=\{h(b), b \in \mathbb{R}\}$, где

$$
u(s)=\left(\begin{array}{ll}
1 & s \\
0 & 1
\end{array}\right), \quad a(\tau)=\left(\begin{array}{cc}
e^{\tau} & 0 \\
0 & e^{-\tau}
\end{array}\right), \quad h(b)=\left(\begin{array}{ll}
1 & 0 \\
b & 1
\end{array}\right) .
$$

Напомним, что диагональная подгруппа $A$ нормализует унипотентные подгруппы $U$ и $H$. Таким образом, $Q=A U$ и $W=A H$ являются двумерными разрешимыми подгруппами в $G$. Относительно левоинвариантной метрики $d_{G}$ слой $x U$ точки $x \in G$ является сжимающимся для правого действия диагональной подгруппы $A$ на $G$. Аналогично, разбиение $G$ на орбиты $x H, x \in G$, образует расширяюшееся слоение. Таким образом, правое действие $A$ на $G$ задает аносовский поток.

Нас, однако, интересует правое действие унипотентной подгруппы $U$ на $G$. Для наглядности будем считать, что направление подгрупшы $U$ вертикально. Тогда трансверсальный лист $x W$ в точке $x \in G$ естественно назвать горизонтальным. Условимся о следующих обозначениях:

$$
A_{\delta}=\{a(\tau),|\tau| \leqslant \delta\}, \quad H_{\delta}=\{h(b),|b| \leqslant \delta\}, \quad U_{\delta}=\{u(s),|s| \leqslant \delta\}
$$

и пусть $W_{\delta}=A_{\delta} H_{\delta}, Q_{\delta}=A_{\delta} U_{\delta}$.

Зафиксируем произвольную точку $x \in G$ и рассмотрим горизонтальную плошадку $x W$. Через время $s$ точка $x$ переместится в точку $x u(s) \in G$. Интуитивно ясно, что если время $s$ не очень велико, а размер $\delta$ площадки $x W_{\delta}$ достаточно мал, то траектория $y U$ произвольной точки $y \in x W_{\delta}$ проткнет горизонтальньй лист $x u(s) W$ в некоторьй момент времени $\alpha(y, s)$. Пусть

$$
y=x a(\tau) h(b) \text { и } y u(\alpha(y, s))=x u(s) a(\tau(y, s)) h(b(y, s)) .
$$

Таким образом, $\alpha(y, s)$ - время, которое требуется точке $y \in x W$, чтобы попасть в горизонтальный лист $x u(s) W$ точки $x u(s)$, а $\tau(y, s)$ и $b(y, s)-A$ - и $H$-координаты точки $y u(\alpha(y, s))$ в горизонтальном листе $x u(s) W$ (причем $\tau(y, 0)=\tau, b(y, 0)=b)$.

Непосредственная проверка показывает, что

$$
\alpha(y, s)=\frac{s}{e^{2 \tau}-s b}, \quad \tau(y, s)=\ln \left(e^{\tau}-s b e^{-\tau}\right), \quad b(y, s)=b\left(1-b s e^{-2 \tau}\right) .
$$

Итак, функции $\alpha(y, s), \tau(y, s), b(y, s)$ определены при $s b<e^{2 \tau}$. Заметим, что если $b=0$, т.е. $y \in x A$, то $\tau(y, s)=\tau, b(y, s)=0, \alpha(y, s)=s / e^{2 \tau}$ и все три функции определены всюду. Если же $b \neq 0$, то существует такое конечное критическое значение $s_{\mathrm{cr}}=s_{\mathrm{cr}}(y)$, что траектория $y U$ не пересекает горизонтальный лист $x u\left(s_{\mathrm{cr}}\right) W$ точки $x u\left(s_{\mathrm{cr}}\right)$, а лиш асимптотически приближается к нему в обоих направлениях (но с разных сторон).

В основе этого факта лежит то обстоятельство, что группа $G$ как многообразие не гомеоморфна 3-мерному векторному пространству и не представляется в виде произведения $U A H$. Поэтому возможно, что элемент $y^{-1} x u\left(s_{\mathrm{cr}}\right)$ не лежит в произведении $U W$ и пересечение $x u\left(s_{\mathrm{cr}}\right) W \cap y U$, таким образом, пусто. 
Разбегание точек $x u(s)$ и $y u(s)$ в метрике $d_{G}$ можно оценить покоординатно. Если в начальный момент времени $s=0$ точка $y$ отстояла от точки $x$ на расстояние $\tau$ в $A$-направлении и на расстояние $b$ в $H$-направлении, то через время $s$ они разошлись на расстояние $\tau(y, s)$ в $A$-направлении, на расстояние $b(y, s)$ в $H$-направлении и еще на расстояние $s-\alpha(y, s)$ в $U$-направлении. Динамика действия $U$ на $G$ такова, что разбегание близких точек происходит в $U$-направлении гораздо быстрее, чем в трансверсальных $A$ - и $H$-направлениях. Например, если $y=x a(\tau)$, то $\tau(y, s) \equiv \tau, b(y, s) \equiv 0$, т.е. в $A$ - и $H$-направлениях никакого разбегания не происходит. Но $\alpha(y, s)=s / e^{2 \tau}$ и поэтому разбегание точек за время $s$ в $U$-направлении пропорционально $s$.

Если $y=x a(\tau) h(b)$, где $b \neq 0$, то полезно оценить поведение функций $\alpha(y, s), \tau(y, s)$ и $b(y, s)$ при $s \rightarrow s_{\text {cr. }}$. Положим $r(s)=1-s / s_{\text {cr. }}$. Тогда $\alpha(y, s)=1 / b r-1 / b, \tau(y, s)=$ $\tau+\ln (r), b(y, s)=b r$. Таким образом, $b(y, s) \rightarrow 0, \tau(y, s)$ имеет особенность порядка $\ln (r)$, а $\alpha(y, s)$ - порядка $1 / r$ при $s \rightarrow s_{\mathrm{cr}}$.

Зафиксируем теперь параметр $0<\theta<1$ и рассмотрим $y \in x W_{\delta}$, где $\delta<\theta / 10$. Пусть $s_{\theta}=s_{\theta}(\tau, b)>0$ - момент времени, при котором разбегание $\tau(y, s)$ точек $x$ и $y=x a(\tau) h(b), b \neq 0$, достигло по абсолютной величине $\theta$, т.е. $\left|\tau\left(y, s_{\theta}\right)\right|=\theta$ (рано или поздно это произойдет для любой точки $y$ с параметром $b \neq 0$ ). В явном виде

$$
\begin{gathered}
s_{\theta}=\frac{e^{\tau}\left(e^{\tau}-e^{-\theta \operatorname{sgn}(b)}\right)}{b}, \quad \alpha\left(y, s_{\theta}\right)=\frac{s_{\theta}}{e^{-\theta \operatorname{sgn}(b)+\tau}}, \\
\tau\left(y, s_{\theta}\right)=-\theta \operatorname{sgn}(b), \quad b\left(y, s_{\theta}\right)=b e^{-\theta \operatorname{sgn}(b)-\tau}
\end{gathered}
$$

где всюду $\operatorname{sgn}(b)-$ сигнатура параметра $b \neq 0$. Если теперь устремить размер $\delta$ начальной площадки $x W_{\delta}$ к нулю, то получим $s_{\theta} \rightarrow+\infty, \alpha\left(y, s_{\theta}\right) / s_{\theta} \rightarrow e^{\theta \operatorname{sgn}(b)}$, $b\left(y, s_{\theta}\right) \rightarrow 0$. Таким образом, чем ближе точки $y$ и $x$, тем большее время требуется им, чтобы разойтись в $A$-направлении на расстояние $\theta$, причем разбегание этих точек в $U$-направлении в этот момент времени стремится к $\infty$, а в $H$-направлении к 0 (при $y \rightarrow x)$. Нам потребуется точная формулировка этого наблюдения - так назьваемое свойство Ратнер, которое играет сушественную роль в доказательстве метрической гипотезы Дани (см. также [R95b]).

ЛЕмма 6.1 ( $R$-свойство орициклического потока). Найдутся такие константы $0<\eta<1, C>1$, что если $0<\theta<1,0<\delta<\theta / 10, y \in x W_{\delta} u\left|\tau\left(y, s_{\theta}\right)\right|=\theta$, причем $|\tau(y, s)|<\theta, 0<s<s_{\theta}$, то $\theta / 2 \leqslant|\tau(y, s)| \leqslant \theta,|b(y, s)| \leqslant C \theta / s_{\theta}$ для всех $s \in\left[(1-\eta) s_{\theta}, s_{\theta}\right]$.

Доказательство. Пусть $x W_{\delta}^{+}=\{y=x a(\tau) h(b),|\tau|<\delta, 0<b<\delta\}$. Функция $s_{\theta}$ монотонно возрастает по $\theta$ для всех $y \in x W_{\delta}^{+}$. Докажем, что найдется $0<\eta<1$ такое, что $s_{\theta / 2} \geqslant(1-\eta) s_{\theta}$ для всех $0<\theta<1, y \in x W_{\delta}^{+}$. Для этого достаточно показать, что $\eta=\inf \left\{1-s_{\theta / 2} / s_{\theta}\right\}>0$, где inf берется по всем $0<\theta<1, y \in x W_{\theta / 10}^{+}$. Ho

$$
1-\frac{s_{\theta / 2}}{s_{\theta}}=\frac{e^{-\theta / 2}-e^{-\theta}}{e^{\tau}-e^{-\theta}} \geqslant \frac{e^{-\theta / 2}-e^{-\theta}}{e^{\theta / 10}-e^{-\theta}}=\frac{e^{\theta / 2}-1}{e^{1.1 \theta}-1}
$$

для всех $|\tau| \leqslant \theta / 10$. Поскольку $\left(e^{\theta / 2}-1\right) /\left(e^{1.1 \theta}-1\right) \rightarrow 10 / 22$ при $\theta \rightarrow 0$, то $\eta>0$. 
Докажем теперь, что $C=\sup \left\{|b(y, s)| s_{\theta} / \theta\right\}<\infty$, где $\operatorname{sup~берется~по~всем~} 0<\theta<1$, $y \in x W_{\theta / 10}^{+},(1-\eta) s_{\theta}<s<s_{\theta}$. Ho

$$
\frac{b(y, s) s_{\theta}}{\theta}=\frac{\left(1-b s e^{-2 \tau}\right)\left(e^{2 \tau}-e^{\tau-\theta}\right)}{\theta} \leqslant \frac{e^{2 \tau}-e^{\tau-\theta}}{\theta} \leqslant \frac{e^{\theta / 5}-e^{-1.1 \theta}}{\theta}
$$

для всех $|\tau| \leqslant \theta / 10$. Поскольку $\left(e^{\theta / 5}-e^{-1.1 \theta}\right) / \theta \rightarrow 13 / 10<\infty$ при $\theta \rightarrow 0$, то $C<\infty$ и все доказано. Нижняя половина $x W_{\theta / 10}^{-}$рассматривается аналогично.

Эта лемма снова отражает то свойство орициклического потока, что разбегание двух близких точек $x$ и $y$ происходит быстрее в направлении самого потока, чем в трансверсальных направлениях. В самом деле, если зафиксировать $0<\theta<1$ и устремить $\delta \rightarrow 0$, то получим $s_{\theta} \rightarrow \infty, s_{\theta}-\alpha\left(y, s_{\theta}\right) \rightarrow \infty,\left|\tau\left(y, s_{\theta}\right)\right|=\theta, b\left(y, s_{\theta}\right) \rightarrow 0$, т.е. при фиксированном разбегании в $A$-направлении разбегание в $U$-направлении стремится к бесконечности, а в $H$-направлении к 0 .

Нам понадобится также следуюший результат о том, что для точки $y \in x W$, достаточно близкой к $x$, функция $\varphi_{y}(s)=\alpha(y, s)$ на отрезке $\left[0, s_{\theta}\right]$ почти не искажает лебеговскую меру $\lambda$ :

ЛЕмма 6.2. Для любого $\varepsilon>0$ найдется $\theta=\theta(\varepsilon)>0$ такое, что для любого у $\in$ $x W_{\theta}$ и любого борелевского множсества $B \subset\left[0, s_{\theta}\right]$ будет $\left|\lambda(B) / \lambda\left(\varphi_{y}(B)\right)-1\right|<\varepsilon$.

ДокАЗАТЕльство. Достаточно доказать, что для любого $\varepsilon>0$ найдется $\theta>0$ такое, что для всех $y \in x W_{\theta}$ и всех $s \in\left[0, s_{\theta}\right]$ будет $\left|\varphi_{y}^{\prime}(s)-1\right|<\varepsilon$. Но $\varphi_{y}(s)=s /\left(e^{2 \tau}-s b\right), \varphi_{y}^{\prime}(s)=e^{2 \tau} /\left(e^{2 \tau}-s b\right)^{2}$. Поскольку функция $\varphi_{y}^{\prime}(s)$ монотонна и $\varphi_{y}^{\prime}(0)=e^{-2 \tau}, \varphi_{y}^{\prime}\left(s_{\theta}\right)=e^{2 \tau+2 \theta \operatorname{sgn}(b)}$, то осталось выбрать $\theta$ из условия $e^{4 \theta}-1<\varepsilon$.

Сформулируем и докажем теорему классификации конечных эргодических мер орициклического потока $\left(\Gamma \backslash G, u_{t}\right)$ на произвольном пространстве $X=\Gamma \backslash G$. Заметим, что для решетки $\Gamma \subset G$ этот результат был получен Дани и Смилли [DS].

Теорема 6.3. Пусть $\Gamma-$-искретная подгруппа в $G=S L(2, \mathbb{R})$ и $\mu$ - эргодическая $U$-инвариантная вероятностная мера на $X=\Gamma \backslash G$. Тогда либо

1) Г-решетка в $G$ и мера $\mu$ является $G$-инвариантной, либо

2) $\mu$ сосредоточена на периодической орбите $U$.

Приступим к доказательству. Далее $d_{X}$ обозначает метрику на $X$, индуцированную левоинвариантной метрикой $d_{G}$ на $G$, и $O_{\varepsilon}(x)=\left\{z \in X: d_{X}(z, x)<\varepsilon\right\}$ обозначает $\varepsilon$-окрестность точки $x \in X$. Пусть

$$
\Lambda=\Lambda(\mu)=\{g \in G: \mu(E)=\mu(E g) \text { для каждого борелевского } E \subset X\} \text {. }
$$

Тогда $\Lambda$ есть стабилизатор меры $\mu$, причем $\Lambda$ является замкнутой подгруппй в $G$, содержашей $U$. Возможны два варианта:

1) $A \not \subset \Lambda$,

2) $A \subset \Lambda$.

Рассмотрим первый случай. Так как $A \not \subset \Lambda$, то $Q=A U \not \subset \Lambda$. 
Лемма 6.4. Если $A \not \subset \Lambda$, то найдется подмножество $Y \subset X$ такое, что $\mu(Y)=1 u Y \cap Y q=\varnothing$ для любого $q \in Q-\Lambda$.

ДокАЗАТЕльство. Найдем вначале подходящее множество для фиксированного элемента $q \in Q-\Lambda$. Положим по определению $\mu_{q}(E)=\mu(E q)$ для любого $q \in Q-\Lambda$. Тогда мера $\mu_{q}$ является $U$-инвариантной эргодической вероятностной мерой, не совпадающей с $\mu$. Поэтому меры $\mu_{q}$ и $\mu$ взаимно сингулярны, т.е. найдется множество $E \subset X$ такое, что $\mu(E)=1, \mu_{q}(E)=\mu(E q)=0$. Тогда если $Y_{q}=E-E q$, то $\mu\left(Y_{q}\right)=1$ и $Y_{q} \cap Y_{q} q=\varnothing$.

Рассмотрим компакт $K \subset Y_{q}$ такой, что $\mu(K) \geqslant 0.99$. Так как $K \cap K q=\varnothing$, то найдется такое $\varepsilon>0$, что $d_{X}(K, K q) \geqslant \varepsilon$. Ввиду эргодичности меры $\mu$ множество $Y_{q, \varepsilon} \subset X$, состоящее из всех точек $x \in X$, для которых

$$
S_{\chi_{K}}(x, t)=\frac{1}{t} \int_{0}^{t} \chi_{K}(x u(s)) d s \rightarrow \mu(K)
$$

имеет полную $\mu$-меру (здесь и далее функция $\chi_{K}$ означает индикатор подмножества $K \subset X)$. Докажем, что $Y_{q, \varepsilon} \cap Y_{q, \varepsilon} q=\varnothing$ для любого $g \in q Q_{\varepsilon}$. Предположим, напротив, что найдутся элемент $g \in q Q_{\varepsilon}$ и точки $x, y \in Y_{q, \varepsilon}$ такие, что $x=y g$. Пусть $g=a(\tau) u(r)$. Тогда $y u(s) g=x u\left(e^{-2 \tau} s\right)$ для любого $s \in \mathbb{R}$. Так как $x, y \in Y_{q, \varepsilon}$, то для достаточно большого $t>1$ мы будем иметь $S_{\chi_{K}}(y, t) \geqslant 0.9$ и $S_{\chi_{K}}\left(x, e^{-2 \tau} t\right) \geqslant 0.9$. Следовательно, непременно найдется значение $s \in[0, t]$, для которого $z=y u(s) \in K$ и $z g=x u\left(e^{-2 \tau} s\right) \in K$. Но $z g=z q p$, где $p \in Q_{\varepsilon}$, и поэтому $d_{X}(z g, z q)<\varepsilon$, причем $z g \in K$ и $z q \in K q$. Противоречие.

Наконец, представим множество $Q-\Lambda$ в виде счетного объединения $Q-\Lambda=$ $\bigcup_{i} Q_{\varepsilon_{i}}\left(q_{i}\right)$ и положим $Y=\bigcap_{i} Y_{q_{i}, \varepsilon_{i}}$. Тогда $\mu(Y)=1$ и $Y \cap Y(Q-\Lambda)=\varnothing$.

Теперь мы готовы закончить рассмотрение первого варианта.

Лемма 6.5. Если $A \not \subset \Lambda$, то найдется точка $x \in X$, для которой $\mu(x U)=1$ и поэтому мера $\mu$ сосредоточена на периодической орбите.

ДокАЗАТЕльство. Пусть $\theta>0$ таково, что $a(\tau) \notin \Lambda$ для любого $0<|\tau|<\theta$ и $\theta \leqslant \theta(0.1)$ (см. лемму 6.2). Константы $C>1, \eta<1$ возьмем из $R$-свойства, а множество $Y \subset X$ - из леммы 6.4. Тогда найдутся компакт $K \subset Y$ и число $\delta>0$ такие, что $\mu(K)>1-\eta / 1000$ и $d_{X}(K, K a(\tau)) \geqslant \delta$ при всех $\theta / 2 \leqslant|\tau| \leqslant \theta$. Ввиду эргодичности меры $\mu$ сушествуют множество $F \subset X$ положительной $\mu$-меры и значение $t_{0} \geqslant 1$ такие, что $S_{\chi_{K}}(x, t) \geqslant 1-\eta / 100$ для всех $x \in F, t \geqslant t_{0}$. Наконец, выберем настолько малое $0<\xi<\theta$, что для любых $x \in G, y \in W(x, \xi)$ будет $s_{\theta} \geqslant 10 t_{0}$ и $C \theta / s_{\theta}<\delta / 10$.

Докажем, что множество $F$ локально содержится в двумерном вертикальном $Q$-листе: $F \cap O_{\varepsilon}(x) \subset x Q_{\xi}$ для каждого $x \in F$. Не ограничивая обшности предположим обратное: найдутся $x, y \in F, y=x a(\tau) h(b),|\tau|<\xi, 0<|b|<\xi$. Мы имеем $s_{\theta}>t_{0}$ и $\alpha\left(y, s_{\theta}\right)>t_{0}$. Поэтому $S_{\chi_{K}}\left(x, s_{\theta}\right) \geqslant 1-\eta / 100$ и, аналогично, $S_{\chi_{K}}\left(y, \alpha\left(y, s_{\theta}\right)\right) \geqslant 1-\eta / 100$. Ввиду леммы 6.2 и выбора $\theta$ функция $\alpha(y, s)$ почти не искажает лебеговскую меру на $\mathbb{R}$ и поэтому найдется хотя бы одно значение $s \in\left[(1-\eta) s_{\theta}, s_{\theta}\right]$ такое, что $x u(s) \in K$ и $y u(\alpha(y, s)) \in K$. Но $y u(\alpha(y, s))=$ $x u(s) a(\tau(y, s)) h(b(y, s))$, причем по $R$-свойству $\theta / 2 \leqslant|\tau(y, s)| \leqslant \theta$ и $|b(y, s)| \leqslant$ $C \theta / s_{\theta} \leqslant \delta / 10$. Так как $x u(s) \in K$, то $x u(s) a(\tau(y, s)) \in K a(\tau(y, s))$. Следовательно, 
$d_{X}(K, K a(\tau(y, s))) \leqslant \delta / 10$, где $\theta / 2 \leqslant|\tau(y, s)| \leqslant \theta$. Полученное противоречие с выбором $\delta$ показывает, что $F \cap O_{\varepsilon}(x) \subset x Q_{\xi}$.

С другой стороны, так как $Q_{\xi} \cap(Q-\Lambda) \subset U$ и $Y \cap Y(Q-\Lambda)=\varnothing$, то $Y \cap x Q_{\xi} \subset$ $x U$ для каждого $x \in Y$. Поскольку $\mu(F)>0$ и $\mu(Y)=1$, то найдется такая точка $x \in F \cap Y$, что $\mu\left(F \cap O_{\varepsilon}(x)\right)>0$ для любого $\varepsilon>0$. В частности, $0<\mu\left(F \cap O_{\xi}(x)\right)<$ $\mu\left(x Q_{\xi}\right)=\mu\left(x Q_{\xi} \cap Y\right)<\mu(x U)$. Следовательно, ввиду эргодичности $\mu(x U)=1$.

Теперь мы приступим к рассмотрению второго варианта. Следуюший результат восходит к Хедлунду, которьй доказывал перемешивание геодезического потока (относительно меры Лиувилля), исходя из эргодичности орициклического.

Лемма 6.6. Если $A \subset \Lambda$, то $A$-действие на $(X, \mu)$ является перемешивающим.

ДокАЗАТЕльство ([R92]). Достаточно показать, что $\int_{X} \varphi(x) f(x a(-\tau)) d \mu \rightarrow 0$, $\tau \rightarrow \infty$, для любых двух ограниченных равномерно непрерьвных функций $\varphi, f$ на $X$ с $\int_{X} f d \mu=0$. Зафиксируем $\varepsilon>0$ и выберем такое $0<\delta<1$, что $|\varphi(x)-\varphi(z)|<\varepsilon$ для любых $x, z \in X, d_{X}(x, z)<\delta$. Ввиду $U$-эргодичности на $(X, \mu)$ найдутся $t_{0}>1$ и $Y \subset X$ такие, что $\mu(Y)>1-\varepsilon$ и $\left|S_{f}(y, t)\right|<\varepsilon$ для всех $y \in Y, t \geqslant t_{0}$. Возьмем настолько большое $\tau_{0}>0$, что $e^{-2 \tau_{0}} t_{0}=\delta$, и пусть $\tau \geqslant \tau_{0}$. Тогда для $Y_{\tau}=Y a(\tau)$ ввиду $A \subset \Lambda$ мы получим $\mu\left(Y_{\tau}\right)=\mu(Y)>1-\varepsilon$. Следовательно,

$$
\begin{aligned}
I(\tau) & =\int_{X} \varphi(x) f(x a(-\tau)) d \mu \\
& =\frac{1}{\delta} \int_{0}^{\delta}\left(\int_{X} \varphi(x u(s)) f(x u(s) a(-\tau)) d \mu\right) d s \\
& =\int_{X}\left(\frac{1}{\delta} \int_{0}^{\delta} \varphi(x u(s)) f(x u(s) a(-\tau)) d s\right) d \mu \\
& =\int_{X} \frac{\varphi(x)}{\delta}\left(\int_{0}^{\delta} f\left(x a(-\tau) u\left(e^{2 \tau} s\right)\right) d s\right) d \mu+\varepsilon_{1} \\
& =\int_{X} \frac{\varphi(x)}{s_{\tau}}\left(\int_{0}^{s_{\tau}} f(x a(-\tau) u(s)) d s\right) d \mu+\varepsilon_{1} \\
& =\int_{X} \varphi(x) S_{f}\left(x a(-\tau), s_{\tau}\right) d \mu+\varepsilon_{1} \\
& =\int_{Y_{\tau}} \varphi(y) S_{f}\left(y a(-\tau), s_{\tau}\right) d \mu+\varepsilon_{1}+\varepsilon_{2}=\varepsilon_{1}+\varepsilon_{2}+\varepsilon_{3} \rightarrow 0,
\end{aligned}
$$

где $s_{\tau}=\delta e^{2 \tau} \geqslant t_{0}$. Здесь оценка на $\varepsilon_{1}$ вытекает из равномерной непрерьвности $\varphi$, на $\varepsilon_{2}-$ из ограниченности $\varphi$ и $f$, а на $\varepsilon_{3}-$ из выбора $Y$.

Последним шагом в доказательстве теоремы является

Лемма 6.7. Пусть $A \subset \Lambda$. Тогда если $\nu$ есть $G$-инвариантная борелевская мера на $X=\Gamma \backslash G$, то $\nu(X)<\infty u \mu=\nu / \nu(X)$. 
ДокАЗАтельство. Рассмотрим произвольную непрерьвную функцию $f$ на $X$ с компактным носителем. Ввиду $A$-эргодичности меры $\mu$ (см. лемму 6.6) множество $C_{f} \subset X$ всех точек $y \in X$, для которых

$$
S_{f, n}(y)=\frac{1}{n} \sum_{i=0}^{n-1} f\left(y a^{-i}\right) \rightarrow f_{\mu}=\int_{X} f d \mu, \quad n \rightarrow \infty,
$$

имеет полную $\mu$-меру. Так как $H$-орбиты являются сжимающимися орициклами для отрицательного направления геодезических, то $d_{X}\left(z a^{-n}, y a^{-n}\right) \rightarrow 0, n \rightarrow+\infty$, для любого элемента $z \in y H$. Поскольку функция $f$ равномерно непрерьвна, то из $S_{f, n}(y) \rightarrow f_{\mu}$ следует $S_{f, n}(z) \rightarrow f_{\mu}, z \in y H$. Следовательно, $C_{f} H=C_{f}$. С другой стороны, мера $\mu$ является $A U$-инвариантной и $\mu\left(C_{f}\right)=1$. Следовательно, $C_{f}$ имеет полную $\nu$-меру. А именно: в окрестности $O(x)=x Q_{\delta / 2} H_{\delta} \cap x H_{\delta / 2} Q_{\delta}$ разложим меру $\mu$ в семейство условных мер $\mu_{y}$ на листах $y Q_{\delta / 2}, y \in x H_{\delta / 2}$. Из $Q$-инвариантности $\mu$ следует, что (почти) каждая $\mu_{y}$ является гладкой, т.е. эквивалентной лебеговской плошади на $y Q_{\delta / 2}$. Все пересечения $C_{f} \cap y Q_{\delta / 2}$ получаются из $C_{f} \cap x Q_{\delta / 2}$ с помошью аналитических отображений, которые переводят множества лебеговской площади 0 в такие же. Поэтому объем $C_{f} \cap O(x)$ равен объему $O(x)$ (подробности см. в [R92]).

Предположим теперь, что функция $f$ вдобавок неотрицательна и $f_{\mu}>0$. Тогда по лемме Фату

$$
f_{\mu} \nu(X)=f_{\mu} \nu\left(C_{f}\right)=\int_{C_{f}} f_{\mu} d \nu \leqslant \lim _{n \rightarrow \infty} \int_{C_{f}} S_{f, n} d \nu=\int_{C_{f}} f d \nu=\int_{X} f d \nu<\infty .
$$

Мы доказали, что $\nu(X)<\infty$, и поскольку последовательность $S_{f, n}$ равномерно ограничена, мы можем применить теорему Лебега о предельном переходе:

$$
f_{\nu}=\int_{X} f d \nu=\int_{C_{f}} f d \nu=\int_{C_{f}} S_{f, n} d \nu \rightarrow \int_{C_{f}} f_{\mu} d \nu=f_{\mu} \nu(X)
$$

и поэтому $f_{\mu}=f_{\nu} / \nu(X)$ для любой неотрицательной непрерывной функции $f$ с компактньм носителем. Следовательно, $\mu=\nu / \nu(X)$, что и требовалось.

\section{§ 7. Классификация эргодических мер унипотентных потоков}

1. Метрическая гипотеза Дани состояла в том, что все вероятностные эргодические меры для действия унипотентных подгрупп $U \subset G$ на однородных пространствах $\Gamma \backslash G$ имеют алгебраическое происхождение. Гипотеза Маргулиса распространяла это утверждение на все подгруппы $U \subset G$, порожденные унипотентными элементами (cм. обзоры $[\mathrm{R} 94,95 \mathrm{~b}],[\mathrm{B}])$. Полное решение гипотезы Дани и "почти полное" гипотезы Маргулиса было получено Мариной Ратнер.

TeOPema 7.1 ([R91a]). Пусть $G$ - группа Ли с дискретной подгруппой $\Gamma \subset G$ и подгруппа $U \subset G$ имеет вид $U=\bigcup_{i=1}^{\infty} u_{i} U_{0}$, где все и $u_{i}$ унипотентны в $G, U / U_{0}$ 
конечно порождена и связная компонента $U_{0}$ порождена однопараметрическими унипотентными подгруппами. Тогда любая $U$-эргодическая вероятностная мера на $\Gamma \backslash G$ является алгебраической.

Основную часть доказательства этой теоремы занимает рассмотрение однопараметрической подгрупшы $U \subset G$. Мы дадим лишь набросок этого сложного доказательства.

ОПРЕДЕЛЕНИЕ 7.2. Пусть $a \in G, x \in G$ и подгруппа $\Gamma \subset G$ дискретна. Скажем, что полуорбита $\left\{\Gamma x a^{n}, n \in \mathbb{Z}^{+}\right\}$сушественно дивергентна, если найдутся элементы $\gamma_{n} \in \Gamma-\{1\}$ такие, что $\left(x a^{n}\right)^{-1} \gamma_{n}\left(x a^{n}\right) \rightarrow 1, n \rightarrow+\infty$.

Легко видеть, что если полуорбита $\Gamma x a^{n}$ сушественно дивергентна, то она является дивергентной (уходит на бесконечность в $\Gamma \backslash G$ ). В самом деле, предположим, что $\Gamma x a^{n_{k}} \rightarrow \Gamma z, n_{k} \rightarrow+\infty$. Тогда найдется последовательность $\theta_{k} \in \Gamma$ такая, что $\theta_{k} x a^{n_{k}} \rightarrow z$. Ho

$$
\left(x a^{n_{k}}\right)^{-1} \gamma_{n_{k}}\left(x a^{n_{k}}\right)=\left(\theta_{k} x a^{n_{k}}\right)^{-1} \theta_{k} \gamma_{n_{k}} \theta_{k}^{-1}\left(\theta_{k} x a_{n_{k}}\right) \rightarrow 1
$$

и поэтому $\theta_{k} \gamma_{n_{k}} \theta_{k}^{-1} \rightarrow 1$. Поскольку Г дискретна и $\gamma_{n_{k}} \neq 1$, мы получили противоречие.

Для элемента $a \in G$ рассмотрим множество $D(a) \subset \Gamma \backslash G$ всех сушественно дивергентных полуорбит. Если $\mu$ - конечная $a$-инвариантная мера на $\Gamma \backslash G$, то по теореме Пуанкаре о возвращении $\mu(D(a))=0$.

Напомним, что элемент $\mathfrak{a} \in \mathfrak{g}$ является полупростым, если оператор $a d_{\mathfrak{a}}$ диагонализуем над $\mathbb{C}$. Если же этот оператор приводится к диагональному виду над $\mathbb{R}$, то элемент $\mathfrak{a} \in \mathfrak{g}$ назьвается $\mathbb{R}$-диагонализуемым.

ОПРЕДЕЛЕНИЕ 7.3. Нильпотентньй элемент $\mathfrak{u} \in \mathfrak{g}$ назьвается оришиклическим, если найдутся $\mathbb{R}$-диагонализуемый элемент $\mathfrak{a} \in \mathfrak{g}$ и нильпотентньй элемент $\mathfrak{u}^{*} \in \mathfrak{g}$ такие, что $\left[\mathfrak{u}, \mathfrak{u}^{*}\right]=\mathfrak{a},[\mathfrak{u}, \mathfrak{a}]=-2 \mathfrak{u},\left[\mathfrak{u}^{*}, \mathfrak{a}\right]=2 \mathfrak{u}^{*}$. Элемент $\mathfrak{a}$ в этом случае назьвается диагональным для $u$. Аналогично, элемент $a=\exp (\mathfrak{a}) \in G$ называется диагональным для орициклического элемента $u=\exp (\mathfrak{u}) \in G$.

По определению элементы $\mathfrak{a}, \mathfrak{u}, \mathfrak{u}^{*}$ порождают подалгебру $s l_{2}(\mathfrak{u}, \mathfrak{a}) \simeq \operatorname{sl}(2, \mathbb{R})$. В свою очередь подгруппы $\exp (\mathbb{R} \mathfrak{a}), \exp (\mathbb{R} \mathfrak{u})$ и $\exp \left(\mathbb{R} \mathfrak{u}^{*}\right)$ порождают подгруппу $S L_{2}(u, a)$ в $G$, локально изоморфную $S L(2, \mathbb{R})$. Отметим, что по лемме Морозова-Джекобсона [Ja] любой нильпотентный элемент в полупростой алгебре Ли является орициклическим. Ясно также, что в произвольной алгебре Ли g нильпотентньй элемент не обязан быть орищиклическим (пример: алгебра g разрешима).

Пусть $G^{-}(a)$ есть сжимаюшаяся орисферическая подгруппа для диагонального элемента $a=\exp (\mathfrak{a}) \in G$. Тогда $u=\exp (\mathfrak{u}) \in G^{-}(a)$. Заметим, что диагональный элемент для орициклического элемента вообще говоря, не единственен. Однако можно доказать (см. [R91a]), что если $a, a^{\prime} \in G$ диагональны для $u \in G$, то $a^{\prime}=c a c^{-1}$ для некоторого $c \in G^{-}(a) \cap Z_{G}(u)$ (где $Z_{G}(u)$ обозначает централизатор $u$ в $G$ ).

Заметим, что если элемент $a \in G$ диагонален для $u \in G$, то множество $D(a)$ существенно дивергентных полуорбит в $\Gamma \backslash G$ инвариантно относительно $u$-сдвига. Поэтому если $\mu$ есть эргодическая вероятностная $u$-инвариантная мера, то либо 
$\mu(D(a))=0$, либо $\mu(D(a))=1$. Кроме того, $D(a)=D\left(\right.$ cac $\left.^{-1}\right)$ для любого $c \in G^{-}(a)$. Следовательно, множество $D(a)$ не зависит от выбора диагонального элемента для $u \in G$.

Пусть $\mu$ есть эргодическая вероятностная мера на $\Gamma \backslash G$ для орищиклического элемента $u \in G$ и $\Lambda=\Lambda(\mu) \subset G$ - ее стабилизатор. Используя $R$-свойство (см. [R95b], где оно обсуждается в общем случае), Марина Ратнер доказала, что альтернатива $\mu(D(a))=0$ или 1 в точности соответствует тому, найдется элемент $c \in G^{-}(a)$ такой, что $c c^{-1} \in \Lambda$ или нет. Если такой элемент $c \in G$ существует, оказьвается возможным доказать, что $c S L_{2}(u, a) c^{-1} \subset \Lambda$ (и, таким образом, подгруппа $\Lambda$ неаменабельна) и мера $\mu$ алгебраична: $\mu\left(x \Lambda_{0}\right)=1$ для некоторой точки $x \in \Gamma \backslash G$. Таким образом, верна

TЕОРема 7.4 ([R91a]). Пусть әлемент $а \in G$ диагонален для орициклического әлемента $и \in G$ и несть и-эргодическая вероятностная мера на $\Gamma \backslash G$. Тогда либо 1) $\mu(D(a))=1$, либо 2) $c S L_{2}(u, a) c^{-1} \subset \Lambda(\mu)$ для некоторого $c \in G^{-}(a) u$ мера $\mu$ алгебраична.

Заметим, что для $G=S L(2, \mathbb{R})$ (см. $\S 6$ ) первая возможность соответствует тому, что геодезический поток не сохраняет меру $\mu$ (а сама мера оказывается сосредоточенной на периодической орбите орицикла). Во втором случае мера $\mu$ является $G$-инвариантной мерой на $\Gamma \backslash G$, индуцированной мерой Хаара на $G$.

Основная метрическая теорема для однопараметрической унипотентной подгруппы $U$ доказывается по следуюшей схеме с использованием индукции по $\operatorname{dim}(G)$. Рассмотрим стабилизатор $\Lambda$ для $U$-эргодической вероятностной меры $\mu$. Возможны два варианта. Если связная компонента $\Lambda_{0}$ аменабельна, то максимальная связная унипотентная в $G$ подгруппа $N \subset \Lambda_{0}$ нормальна в $\Lambda_{0}$ и содержит $U$. В этом случае с использованием обобщенного $R$-свойства для унипотентной подгруппы $N \subset G$ удается доказать алгебраичность $\mu$. Если же $\Lambda_{0}$ неаменабельна, она содержит некоторьй орищиклический элемент $h$, диагональньй элемент $a$ и подгруппу $S L_{2}(h, a)$. Так как $a \in \Lambda$, то $\mu(D(a))=0$. Следовательно, по теореме $7.4 \mu$-почти всякая эргодическая компонента для орищиклического элемента $h \in G$ алгебраична. Рассмотрение факторпространства по модулю этого разбиения позволяет снизить размерность группы Ли и применить аргумент индукции.

Для того чтобы доказать теорему 7.1 в случае $\operatorname{dim}(U)>1$, напомним следуюший результат.

ТеОРема 7.5 (Пью-Шуб, [PS]). Пусть $U-$ связная абелева группа Ли с эргодическим действием на вероятностном пространстве $(X, \mu)$. Тогда почти всякий әлемент $U$ (относительно меры Хаара на U) действует на $(X, \mu)$ әргодически.

M. Ратнер обобщила этот результат на класс связных нильпотентных групп Ли (см. также [Da89]), доказав таким образом метрическую теорему для связных унипотентных подгрупп $U \subset G$. Отсюда уже нетрудно вывести теорему 7.1 и для связных подгруп $U \subset G$, порожденных унипотентными элементами. В самом деле, такая группа $U$ имеет вид $U=P M$, где подгруппа $M \subset G$ унипотентна, а $P \subset G$ полупроста и не 
имеет компактных множителей. Пусть $V \subset P$ - максимальная связная унипотентная подгрупша. Тогда $V M$ является максимальной связной унипотентной подгруппой в $U$ и нормальная в $U$ подгруппа Мура для $V M$ совпадает с $U$. Но в силу феномена Маутнера С.1 любая $U$-эргодическая конечная мера является $V M$-эргодической, и мы можем найти эргодическую однопараметрическую подгруппу из $V M$.

Случай несвязной подгруппы $U$, порожденной унипотентньми элементами, технически более сложен. В полном объеме доказательство гипотезы Маргулиса было недавно закончено Шахом.

ТеОРема 7.6 ([Sн97]). Пусть $G$ - группа Ли с замкнутой подгруппой $\Gamma \subset G$ $u$ подгруппа $U \subset G$ порождена унипотентными элементами. Тогда любая $U$-эргодическая вероятностная мера на $\Gamma \backslash G$ является алгебраической.

2. Как видно из теоремы 7.6, гипотеза Маргулиса доказана для произвольной замкнутой подгруппы $\Gamma \subset G$ и любой (возможно, несвязной) групшы $G$. В явном виде требование дискретности Г было устранено Витте [Wi94] с использованием теоремы плотности В.3, но по существу имелось еще в работе автора [St88], где проведена редукция теории однородных потоков к случаю дискретной подгрупшы изотропии.

Изложим идею этой редукции для связной односвязной группы $G$, используя конструкцию расшепления Мальцева $[\mathrm{M}]$ для разрешимых груп. ${ }^{13}$ Пусть $G=L \cdot R$ - разложение на радикал $R$ и полупростую подгруппу Леви $L$. Согласно Мальцеву (см. [M] или $[\mathrm{BM}]) R$ вкладывается в вешественно-алгебраическую группу $A(R)=A\left(T_{R}\right) \cdot R$, причем найдется такой выбор абелевой редуктивной подгруппы $A\left(T_{R}\right) \subset \operatorname{Aut}(R)$, что ее действие на $R$ коммутирует с действием $L$. Кроме того, любой $\mathrm{Ad}$-унипотентный элемент в $R$ унипотентен и как элемент $A(R)$. Пусть $A(G)=\left(L \times A\left(T_{R}\right)\right) \cdot R, Z(G)$ - центр $G$ и $Z=Z(G) \cap L$. Предположим вначале, что $Z=1$. Тогда групша $A(G)$ является вешественно-алгебраической и любой $\mathrm{Ad}$-унипотентный элемент в $G$ унипотентен в $A(G)$. Обозначим через $A(H) \subset A(G)$ алгебраическую оболочку любой подгрупшы $H \subset A(G)$.

Пусть теперь замкнутая подгруппа $U$ порождена унипотентными элементами и $\mu$ является $U$-инвариантной эргодической конечной мерой на $\Gamma \backslash A(G)$. Пусть $X=$ $\left\{g \in A(G): g U g^{-1} \subset A(\Gamma)\right\}$. Тогда $\Gamma X=X$. Докажем, что мера $\mu$ сосредоточена на замкнутом подмножестве $\Gamma X \subset \Gamma \backslash G$. В самом деле, если $g \notin X$, то найдется унипотентньй элемент $u \in U$ такой, что $g g^{-1} \notin A(\Gamma)$. Алгебраическая оболочка подгрупшы $\left\{g u^{k} g^{-1}, k \in \mathbb{Z}\right\}$ является однопараметрической унипотентной подгруппой. Так как произведение двух алгебраических подгрупп локально замкнуто и $g \notin X$, то орбита $\left\{A(\Gamma) g u^{k}, k \in \mathbb{Z}\right\} \subset A(\Gamma) \backslash A(G)$ локально замкнута и непериодична. В этом случае орбита $\left\{\Gamma g u^{k}, k \in \mathbb{Z}\right\} \subset \Gamma \backslash A(G)$ также локально замкнута и непериодична. Но множество таких орбит имеет $\mu$-меру 0 , поскольку $\mu$-почти все орбиты подгрупшы $\left\{u^{k}, k \in \mathbb{Z}\right\}$ на $\Gamma \backslash G$ являются рекуррентньми. Так как в $U$ найдется плотная счетная подгруппа, порожденная унипотентньми элементами, то $\operatorname{supp}(\mu) \subset \Gamma X$.

Итак, мера $\mu$ сосредоточена на объединении $U$-инвариантных замкнутых подмножеств вида $X(g)=\Gamma \backslash A(\Gamma) g$, где $g \in X$. Ввиду эргодичности меры $\mu$ найдется такой элемент $g \in X$, что $\mu$ сосредоточена на $X(g) \subset \Gamma \backslash A(G)$. Поток $(X(g), U)$ изоморфен

\footnotetext{
${ }^{13}$ Впервые для изучения однородных потоков ее применил Ауслендер [AG], а в отечественной литературе - Сафоронов [Saf].
} 
потоку $\left(\Gamma \backslash A(\Gamma), g U g^{-1}\right)$. Но связная компонента $\Gamma_{0}$ нормальна в $A(\Gamma)$, и мы можем теперь воспользоваться классификацией эргодических мер на однородном пространстве $\left(\Gamma / \Gamma_{0}\right) \backslash\left(A(\Gamma) / \Gamma_{0}\right)$ с дискретной подгруппой изотропии.

В обшем случае можно рассмотреть эпиморфизм $\psi: A(G) \rightarrow A(G) / Z=(L / Z \times$ $\left.A\left(T_{R}\right)\right) \cdot R$ и имитировать структуру алгебраической групшы на $A(G)$, беря для $H \subset G$ в качестве "алгебраической" оболочки $A(H) \subset A(G)$ группу $\psi^{-1}(A(\psi(H)))_{0} H$ (пользуясь тем, что $A(G) / Z$ является "настояшей” алгебраической группой). Легко проверить, что все предыдущие рассуждения проходят дословно.

3. В заключение мы изложим схему доказательства метрической теоремы для алгебраических груп способом, предложенньм Маргулисом и Томановым [MT94].

Пусть $G \subset G L(n, \mathbb{R})$ - алгебраическая группа над $\mathbb{R}$ с дискретной подгруппой $Г$. Если элемент $g \in G$ нормализует связную подгруппу $A \subset G$, через $\alpha(g, A)$ обозначим коэффициент искажения меры Хаара $m$ на $A$ элементом $g$ (т.е. $m\left(g^{-1} X g\right)=$ $\alpha(g, A) m(X)$ для любого измеримого $X \subset A)$. Скажем, что элемент $s \in G$ класса $\mathscr{A}$, если его алгебраическая оболочка в $G$ одномерна и диагонализуема над $\mathbb{R}$. Для $s \in G$ класса $\mathscr{A}$ определим $W^{+}=G^{+}(s), W^{-}=G^{-}(s), Z=Z_{G}(s)$. Элемент $s^{-1}$ расширяет $W^{-}$и поэтому $\alpha\left(s^{-1}, V\right)>1$ для любой $s$-инвариантной подгруппы $V \subset W^{-}$. Следующая оценка энтропии важна сама по себе и значительно упрошает доказательство метрической теоремы.

ТЕОРема 7.7 ([МТ94]). Предположим, что $\mu$ - s-инвариантная әргодическая вероятностная мера на $\Gamma \backslash G u V \subset W^{-}$нормализуется элементом s класса $\mathscr{A}$. Пусть $h=h(s, \mu)$ - энтропия $\mu$ по отношению $\kappa s$ u $\alpha=\alpha\left(s^{-1}, V\right)$. Тогда

1) если $\mu$ является $V$-инвариантной, то $h \geqslant \log _{2} \alpha$,

2) если найдется множество $M \subset \Gamma \backslash G$ такое, что $\mu(M)=1$ и $M \cap$ $x W^{-} \subset x V$ для всех $x \in M$, то $h \leqslant \log _{2} \alpha$ и равенство влечет $V$-инвариантность меры $\mu$.

Заметим, что доказательство этой теоремы использует результаты работы [LY].

Теперь пусть $U \subset W^{+}$- унипотентная алгебраическая подгруппа. В [MT94] важную роль играет так назьваемое квазирегулярное отображение $\varphi: U \rightarrow N_{G}(U)$. Приведем идею его построения (cp. [R90b,c]). Предположим для простоты, что $s$ нормализует $U$. Пусть $V \subset W^{+}-s$-инвариантное дополнение к $U$ в $W^{+}$. Тогда произведение $L=V Z W^{-}$является сечением для $U \backslash G$ (т.е. локально $L \cap U=1$ и $G=U L$ ). Зафикисируем последовательность $g_{n} \in G-N_{G}(U)$, сходяшуюся к 1 , и определим отображение $\tilde{\varphi}_{n}: U \rightarrow L$ по правилу $g_{n} u \in U \tilde{\varphi}_{n}(u)$. По теореме Шевалле [Нu] найдутся (правое) действие $G$ на линейном пространстве $E$ и вектор $q \in E$ такие, что $U=\{h \in G: q \circ h=q\}$. Зафиксируем относительно компактные окрестности $A=O(1) \subset U$ и $D=O(q) \subset E$. Положим $A_{n}=s^{-n} A s^{n}$ и определим целые числа $r_{n}$ по правилу: $q \circ\left(g_{n} A_{r_{n}}\right) \not \subset D$ и $q \circ\left(g_{n} A_{k}\right) \subset D$ для всех $k<r_{n}$. Теперь определим $\varphi_{n}: U \rightarrow L$ следуюшим образом: $\varphi_{n}(u)=\tilde{\varphi}_{n}\left(s^{-r_{n}} u s^{r_{n}}\right)$. Легко видеть что отображения $\varphi_{n}^{\prime}: U \rightarrow E$, определенные как $\varphi_{n}^{\prime}(u)=q \circ \varphi_{n}(u)$, являются полиномами равномерно ограниченных степеней. Так как $\varphi_{n}^{\prime}\left(A_{-1}\right) \subset D$ и $\varphi_{n}^{\prime}(A) \not \subset D$, мы можем считать, что $\varphi_{n}^{\prime}$ сходится к некоторому непостоянному полиномиальному отображению $\varphi^{\prime}: U \rightarrow E$. В то же самое время $\varphi_{n}$ сходится к некоторому отображению 
$\varphi: U \rightarrow L$. Нетрудно проверить, что $\operatorname{Im}(\varphi) \subset N_{G}(U)$ и группа $F \subset G$, порожденная $U$ и $\operatorname{Im}(\varphi)$, достаточно велика: факторгруппа $U \backslash F$ некомпактна.

ПримеР 7.8. Рассмотрим $G=S L(2, \mathbb{C})$ как $\mathbb{R}$-алгебраическую группу. Введем обозначения:

$$
s=\left(\begin{array}{cc}
\sqrt{2} & 0 \\
0 & 1 / \sqrt{2}
\end{array}\right), U_{t}=\left(\begin{array}{ll}
1 & 0 \\
t & 1
\end{array}\right), V_{t}=\left(\begin{array}{cc}
1 & 0 \\
i t & 1
\end{array}\right), U_{t}^{-}=\left(\begin{array}{cc}
1 & t \\
0 & 1
\end{array}\right), V_{t}^{-}=\left(\begin{array}{cc}
1 & i t \\
0 & 1
\end{array}\right) .
$$

Ясно, что $W^{+}=U_{\mathbb{R}} \times V_{\mathbb{R}}, W^{-}=U_{\mathbb{R}}^{-} \times V_{\mathbb{R}}^{-}, L=V_{\mathbb{R}} Z W^{-}$, где $Z$ - подгруппа диагональных матриц. Пусть $e^{\prime}, e^{\prime \prime}$ - стандартный $\mathbb{C}$-базис в $\mathbb{C}^{2}$. Тогда $e_{1}=e^{\prime}, e_{2}=i e^{\prime}$, $e_{3}=e^{\prime \prime}, e_{3}=i e^{\prime \prime}$ образуют $\mathbb{R}$-базис. Обозначим через $\rho$ стандартное (правое) действие $G$ на $\mathbb{C}^{2}$ и определим $\rho^{\prime}=\rho \oplus(\rho \wedge \rho)$. Тогда для $q=e_{1} \oplus\left(e_{1} \wedge e_{3}\right)$ мы получим $U=\left\{h \in G: q \circ \rho_{h}^{\prime}=q\right\}$. Легко проверить, что следуюшее удовлетворяет нашему построению:

$$
g_{n}=\left(\begin{array}{cc}
1 & 1 / n \\
0 & 1
\end{array}\right), \quad r_{n}=\log _{2} n, \varphi\left(U_{t}\right)=\left(\begin{array}{cc}
1+t & 0 \\
0 & 1 /(1+t)
\end{array}\right) .
$$

Кроме того, есть и другая комбинация:

$$
g_{n}=\left(\begin{array}{cc}
1 & i / n \\
0 & 1
\end{array}\right), \quad r_{n}=\frac{1}{2} \log _{2} n, \quad \varphi\left(U_{t}\right)=\left(\begin{array}{cc}
1 & 0 \\
-i t^{2} & 1
\end{array}\right) .
$$

Более обшим образом: легко проверить, что $\operatorname{Im}(\varphi) \subset W^{+}$при условии $g_{n} \in$ $V^{-} W^{+} Z-W^{+} Z$.

В дальнейшем будем говорить, что последовательность $\left\{g_{n}\right\}$ удовлетворяет условию ( $\star$ если последовательность $\left\{s^{r_{n}} g_{n} s^{-r_{n}}\right\}$ относительно компактна (для $S L(2, \mathbb{C})$ это всегда так).

Пусть $\mu$ есть $U$-инвариантная эргодическая вероятностная мера на $\Gamma \backslash G$. Еще одно понятие, используемое в [MТ94], это множество равномерной сходимости для последовательности $\left\{A_{n}\right\}$ (cp. [R90b,c]). Это - такое множество $M \subset \Gamma \backslash G$, что для любых $\varepsilon>0$ и $f \in C_{c}(\Gamma \backslash G)$ найдется $N=N(\varepsilon, f)$, для которого

$$
\left|\frac{1}{m\left(A_{n}\right)} \int_{A_{n}} f(x u) d m_{u}-\int_{\Gamma \backslash G} f d \mu\right|<\varepsilon \text { для всех } x \in M, \quad n>N,
$$

где $m$ - мера Хаара на $U$ (заметим, что множества $A_{n}$ растут достаточно равномерно во всех направлениях и мы можем применить аналог эргодической теоремы Биркгофа-Хинчина к усредняюшей последовательности $\left.\left\{A_{n}\right\} \subset U\right)$. В [MT94] доказано, что для каждого $\delta>0$ найдется множество $M_{\delta}$ равномерной сходимости для $A_{n}=s^{-n} A s^{n}$ с $\mu\left(M_{\delta}\right)>1-\delta$ независимо от выбора относительно компактного открытого подмножества $A \subset U$. Использование квазирегулярных отображений $\varphi: U \rightarrow N_{G}(U)$ для доказательства метрической теоремы основано на следуюшем утверждении. 
Лемма 7.9 ([МТ94]). Пусть $M-$ множество равномерной сходимости для любой последовательности $A_{n} \subset U$ указанного вида и допустим, что $x_{n} \in M$ сходится $\kappa x \in M, g_{n} \in G-N_{G}(U)$ сходится $\kappa 1 \in G$ и удовлетворяет условию $(\star)$ и $x_{n} g_{n} \in M$ для всех $n$. Тогда мера $\mu$ является $\operatorname{Im}(\varphi)$-инвариантной.

Скажем, что мера $\mu$ плотна по Зарисскому в $\Gamma \backslash G$, если не найдется собственной алгебраической подгруппы $P \subset G$ такой, что $U \subset P$ и $\mu(x P)=1$ для некоторого $x \in \Gamma \backslash G$. Для $M \subset \Gamma \backslash G$ определим $\Psi(M)=\{g \in G: M g \cap M \neq \varnothing\}$. Пусть $A, B, C, D$ - алгебраические подгруппы в $G$, причем $D \subset A B$. Скажем, что произведение $A B$ трансверсально к $C$, если локально $G=C A B$ и $A B \cap C=1$. Основной инструмент для использования леммы 7.9 состоит в следующем.

ЛЕмма 7.10 ([МТ94]). Пусть $\mu$ плотна по Зарисскому в $\Gamma \backslash G$ и инвариантна относительно $C$. Предположим, что произведение $A B$ трансверсально $к C$. Тогда найдется $\delta>0$ такое, что для любого измеримого множества $M \subset \Gamma \backslash G$ с $\mu(M)>1-\delta$ найдется последовательность $g_{n} \in \Psi(M) \cap(A B-D)$, сходящаяся $\kappa 1 \in G$.

Предположим теперь, что мы хотим доказать алгебраичность эргодической $U$-инвариантной меры $\mu$ на $\Gamma \backslash G$ для некоторой унипотентной алгебраической подгруппы $U \subset G$. Ясно, что, не ограничивая обшности, меру н можнн считать плотной nо Зарисскому. Другое допушение состоит в том, что $U$ не является нормальной в $G$ (иначе доказательство почти тривиально). Наконец, без ограничения общности мы допустим, что подгруппа $U$ максимальна среди алгебраических унипотентных nодгрупn, сохраняющ, $\mu$. Нам нужно доказать, что мера $\mu$ является $G$-инвариантной.

С этого момента мы опять для простоты предположим, что $U \subset W^{+}$для некоторого элемента $s \in G$ класса $\mathscr{A}$. Рассмотрим замыкание по Зарисскому $X \subset G$ произведения $U Z W^{-}$и введем подгруппу $U^{-}=\left\{g \in W^{-}: g X=X\right\}$, которая будет играть роль “противоположной” к $U$. Весьма деликатный анализ локальной структуры меры $\mu$, использующий идеи Ратнер, дает нам следующую информацию:

Лемма 7.11 ([МТ94]). Предположим, что s сохраняет меру $\mu$. Тогда найдется мнохсество полной меры $M \subset \Gamma \backslash G$ такое, что $M \cap x W^{-} \subset x U^{-} \partial л я$ всех $x \in M$.

Мы окончим доказательство на примере группы $G=S L(2, \mathbb{C})$. Достаточно доказать, что мера $\mu$ является $W^{+}$-инвариантной (что противоречит максимальности $U$ ). Пусть $D$ и $S$ - одномерные подгрупшы диагональных матриц соответственно с вешественными и комплексными элементами абсолютной величины 1 . Тогда $Z=D \times S$ и $N_{G}(U)=W^{+} D$. Пусть $M_{\delta}$ - множество равномерной сходимости для последовательности $A_{n} \subset U$ с $\mu\left(M_{\delta}\right)>1-\delta$. Из леммы 7.10 следует, что для достаточно малого $\delta>0$ найдется последовательность $g_{n} \in \Psi\left(M_{\delta}\right) \cap\left(V Z W^{-}-V D\right)$, сходяшаяся к 1. Из леммы 7.9 мы получим квазирегулярное отображение $\varphi: U \rightarrow N_{G}(U)$ такое, что $\operatorname{Im}(\varphi)$ сохраняет $\mu$. Пусть $s^{\prime} \in D$ - элемент класса $\mathscr{A}$, порожденньй $U$ и $\operatorname{Im}(\varphi)$ и расширяющий $U$. Из феномена Маутнера следует $s^{\prime}$-эргодичность меры $\mu$. Применяя лемму 7.11 и теорему 7.7, мы видим, что

$$
\log _{2} \alpha\left(s^{\prime}, U\right) \leqslant h\left(s^{\prime}, \mu\right) \leqslant \log _{2} \alpha\left(s^{\prime-1}, U^{-}\right)=\log _{2} \alpha\left(s^{\prime}, U\right) .
$$


Теперь из теоремы 7.7 следует $U^{-}$-инвариантность $\mu$. Наконец, возьмем трансверсальное к $U^{-}$произведение $V^{-}\left(Z W^{+}\right)$и, используя лемму 7.10 , построим последовательность $g_{n} \in \Psi(M) \cap\left(V^{-} Z W^{+}-Z W^{+}\right)$, сходяшуюся к 1 для некоторого множества $M$ равномерной сходимости по отношению к $\left\{A_{n}\right\} \subset U$. Тогда мы получим квазирегулярное отображение $\varphi: U \rightarrow W^{+}$, и в силу леммы 7.9 мера $\mu$ будет $W^{+}$-инвариантной.

\section{§. Равномерная распределенность унипотентных траекторий}

Приступим к рассмотрению топологической гипотезы Рагунатана; см. также [Ma91b], [R94,95b], [B].

1. Пусть $u_{\mathbb{R}}=\left\{u_{t}, t \in \mathbb{R}\right\}$ - однопараметрическая подгруппа в $G$, подгруппа $\Gamma$ дискретна в $G$ и $x \in \Gamma \backslash G$. Скажем, что траектория $x u_{\mathbb{R}}$ равномерно распределена (в своем замыкании), если найдется замкнутая подгруппа $H \subset G$ такая, что замкнутая орбита $x H \subset \Gamma \backslash G$ имеет $H$-инвариантную вероятностную меру $\nu_{H}$ и для любой функции $f \in C_{c}(\Gamma \backslash G)$ (т.е. непрерьвной функции с компактным носителем) выполнено

$$
\frac{1}{T} \int_{0}^{T} f\left(x u_{t}\right) d t \rightarrow \int_{\Gamma \backslash G} f d \nu_{H}, \quad T \rightarrow \infty
$$

Имеет место

Tеорема 8.1 ([R91b]). Если $\Gamma$ - решетка в связной әруппе Ли $G$, то каждая унипотентная траектория в $\Gamma \backslash G$ равномерно распределена.

Очевидно, что если орбита $x u_{\mathbb{R}}$ равномерно распределена в $x H$, то $\overline{x u_{\mathbb{R}}}=x H$. Поэтому гипотеза Рагунатана для однопараметрических траекторий вытекает из их равномерной распределенности. Это важное свойство унипотентных потоков было впервые доказано М. Ратнер в [R91b]. Одновременно Н. Шах [Sh91] доказал его частньй случай (группа $G$ полупроста, $\operatorname{rank}_{\mathbb{R}} G=1$ и подгруппа $u_{\mathbb{R}}$ регулярна). Оба доказательства опираются на метрическую теорему Ратнер. Изложим вначале общую часть этих доказательств.

Пусть $\Gamma$ - произвольная дискретная подгруппа в $G$ и $X=\Gamma \backslash G$. Рассмотрим сингулярное множество $Y \subset X$ тех точек $x \in X$, для которых существует собственная подгруппа $H \subset G$ такая, что $u_{\mathbb{R}} \subset H$ и замкнутая орбита $x H$ несет на себе вероятностную $H$-инвариантную меру. Используя индукцию по $\operatorname{dim}(G)$, достаточно предположить, что $Y \neq X$, и доказать равномерную распределенность орбит для точек $x \in X-Y$. Пусть $\bar{X}=X \cup \infty$ - одноточечная компактификация и $\mu_{T}, T>0,-$ вероятностная мера на $\bar{X}$, заданная по правилу

$$
\int_{X} f d \mu_{T}=\frac{1}{T} \int_{0}^{T} f\left(x u_{t}\right) d t
$$

Поскольку множество вероятностных мер на компакте само является компактным по отношению к *-слабой топологии, то для доказательства равномерной распределенности орбиты $x u_{\mathbb{R}}$ точки $x \in X-Y$ достаточно показать, что если $\mu_{T_{i}} \rightarrow \mu$ при $T_{i} \rightarrow \infty$, то $\mu$ является $G$-инвариантной вероятностной мерой на $X$. 
Нетрудно видеть, что предельная мера $\mu$ является $u_{\mathbb{R}}$-инвариантной. Рассмотрим разложение меры $\mu$ на $u_{\mathbb{R}}$-эргодические компоненты. По метрической теореме Ратнер все они алгебраичны. Предположим, мы знаем, что $\mu(\infty)<1$ и $\mu(Y)=0$. Тогда $\mu(X)>0$ и найдется эргодическая компонента меры $\left.\mu\right|_{X}$, не сосредоточенная на $Y$. Из алгебраичности такой меры следует ее $G$-инвариантность и потому Г является решеткой в $G$. По теореме Дани D.8 унипотентные траектории на однородном пространстве конечного объема проводят почти все свое время в некотором компактном подмножестве. Это означает, что $\mu(X)=1$, и поэтому сама мера $\mu$ является $G$-инвариантной.

Что касается условия $\mu(\infty)<1$, то оно может и не вьполняться (например, если орбита $x u_{\mathbb{R}}$ уходит на бесконечность в $\left.\Gamma \backslash G\right)$. Однако, если $\Gamma-$ решетка в $G$, то по теореме Дани заведомо $\mu(\infty)=0$ и беспокоиться не о чем. Условие же $\mu(Y)=0$ вьполняется всегда, если $x \notin Y$, и доказательство этого факта является далеко не тривиальным. Имеются два способа для установления этого равенства. Способ Ратнер, как и доказательство метрической теоремы, использует $R$-свойство для унипотентных траекторий. Наше последуюшее изложение следует работе Дани и Маргулиса [DM93], которая основана на изучении поведения унипотентных траекторий вблизи сингулярного множества $Y \subset X$. Подобная идея использовалась ими еше в [DM90a], где была установлена гипотеза Рагунатана для регулярных унипотентных подгрупп в $G=S L(3, \mathbb{R})$. Затем эта идея была развита в работе Шаха [Sh91]. Наш выбор объясняется тем, что данный подход позволяет доказать более сильный результат. Всюду далее $l$ обозначает лебеговскую меру на $\mathbb{R}$.

ТЕОРема 8.2 ([DM93]). Пусть $\Gamma$ - дискретная подгруппа в группе Ли $G$, подгруппа $u_{\mathbb{R}}$ унипотентна в $G, Y \subset \Gamma \backslash G$ - сингулярное множсество для $u_{\mathbb{R}} u x \notin Y$. Тогда для любого $\varepsilon>0$ найдется такая окрестность $\Omega$ сингулярного множесmва $Y \subset \Gamma \backslash G$, ито

$$
l\left\{t \in[0, T]: x u_{t} \in \Omega\right\} \leqslant \varepsilon T \quad \text { для всех } T \geqslant 0 .
$$

Таким образом, нам предстоит доказать, что фиксированная унипотентная траектория проводит лишш малую часть времени внутри (достаточно малой) окрестности сингулярного множества $Y \subset X$. Следствием этой теоремы является равенство $\mu(Y)=0$ для предельной меры $\mu$, построенной по траектории $x u_{\mathbb{R}}, x \in X-Y$. Здесь важно то, что сама теорема имеет силу для любой дискретной подгрупшы $\Gamma \subset G$. Таким образом, замыкание унипотентной траектории заведомо является алгебраическим при условии $\mu(X)>0$, и мы вьвели важное следствие (в [DM93] в явном виде оно не сформулировано и было сообщено автору Шахом).

ТеОРема 8.3. Пусть $\Gamma$ - дискретная подгруппа в $G$, подгруппа $u_{\mathbb{R}} \subset G$ унипотентна и $x \in \Gamma \backslash G$. Предположим, что найдутся $\varepsilon>0$ и компакт $K \subset \Gamma \backslash G$ такие, что

$$
l\left\{t \in[0, T]: x u_{t} \in K\right\}>\varepsilon T \quad \text { для каждого } T>0 .
$$

Тогда замыкание $\overline{x u_{\mathbb{R}}}$ алгебраично, а сама траектория равномерно распределена.

Этот результат доказьвает, в частности, гипотезу Ратнер о том, что компактное замькание унипотентной траектории на $\Gamma \backslash G$ обязано быть алгебраическим всегда, 
если подгруппа $\Gamma \subset G$ дискретна. Для произвольных же подгрупп $\Gamma \subset G$ компактное замькание унипотентной траектории может не являться многоообразием. В самом деле, пусть $G=S L(2, \mathbb{R}) \times \mathbb{Z} \backslash \mathbb{R}, g_{\mathbb{R}}-$ диагональная подгруппа в цилиндре $U \times \mathbb{Z} \backslash \mathbb{R}$, где $U \subset S L(2, \mathbb{R})$ - унипотентная подгрупа строго верхнетреугольных матриц. Если теперь $B \subset S L(2, \mathbb{R})$ - двумерная борелевская подгруппа верхнетреугольных матрищ, то пространство $B \backslash G$ диффеоморфно двумерному тору, в котором есть одна периодическая $g_{\mathbb{R}}$-орбита, а все остальные локально замкнуты и наматьваются на периодическую в обоих направлениях.

2. Приступим к доказательству теоремы 8.2, следуя [DM93]. Вначале рассмотрим подробнее структуру сингулярного множества $Y \subset X$, ассоциированного с унипотентной подгруппой $u_{\mathbb{R}} \subset G$. Пусть $\mathscr{H}$ обозначает семейство всех собственных связных подгрупп $H \subset G$ таких, что $\Gamma \cap H$ является решеткой в $H$, плотной по Зарисскому в $H$ относительно Ad-представления групшы $G$. Кроме того, для любых двух замкнутых подгрупш $H, W \subset G$ рассмотрим аналитическое множество

$$
X(H, W)=\left\{g \in G: g W g^{-1} \subset H\right\}
$$

Лемма 8.4. $Y=\bigcup_{H \in \mathscr{H}} \Gamma X\left(H, u_{\mathbb{R}}\right) \subset \Gamma \backslash G$.

ДоказАтельство. Пусть $\Gamma g \in \Gamma X\left(H, u_{\mathbb{R}}\right)$, где $H \in \mathscr{H}$. Тогда подпространство $\Gamma H g \subset \Gamma \backslash G$ замкнуто, имеет конечньй объем и содержит траекторию $\Gamma g u_{\mathbb{R}}$. Следовательно, $\Gamma g \in Y$.

Обратно, пусть $\Gamma g \in Y$. Тогда найдется собственная связная подгруппа $H \subset G$ такая, что $u_{\mathbb{R}} \subset H$ и замкнутое подпространство $\Gamma g H$ имеет конечный объем. Следовательно, $g u_{\mathbb{R}} g^{-1} \subset g H g^{-1}$ и $\Gamma \cap g g^{-1}$ является решеткой в $g H^{-1}$. Пусть $H^{\prime}$ - подгруппа в связной группе Ли $g \mathrm{Hg}^{-1}$, порожденная всеми $\mathrm{Ad}$-унипотентньми в $G$ однопараметрическими подгруппами. Тогда $g u_{\mathbb{R}} g^{-1} \subset H^{\prime}, H^{\prime}$ нормальна в $g H^{-1}$ и по версии Дани теоремы плотности Бореля В.3 алгебраическое замькание подгруппы $\operatorname{Ad}\left(\Gamma \cap g g^{-1}\right)$ содержит $\operatorname{Ad}\left(H^{\prime}\right)$. Поэтому если $F=\left(\overline{\Gamma H^{\prime}}\right)_{0}$, то $g u_{\mathbb{R}} g^{-1} \subset F$ и $\Gamma \cap F$ является решеткой в $F$, плотной по Зарисскому в $\mathrm{Ad}$-представлении $G$. Следовательно, $g \in X\left(F, u_{\mathbb{R}}\right)$, где $F \in \mathscr{H}$.

Tеорема 8.5. Семейство $\mathscr{H}$ счетно.

Заметим, что этот важный результат впервые появился в работе Ратнер [R91a] и был использован для доказательства теоремы 8.1, а также (в частном случае) у Шаха [Sh91].

Мы приведем основную идею доказательства из [DM93]. Предположим для простоты, что $\mathrm{Ad}$-представление группы $G$ является точньм и образ $\operatorname{Ad}(G)$ замкнут относительно евклидовой топологии в алгебраической групе $\operatorname{Aut}(\mathfrak{g})$. Пересечение $\Gamma \cap H$ для $H \in \mathscr{H}$, будучи решеткой в связной групе Ли $H$, является конечно-порожденной группой. Ввиду счетности Г число ее конечно-порожденных подгрупп также счетно. Поэтому достаточно показать, что для данной конечно-порожденной подгруппы $\Delta \subset \Gamma$ найдется не более чем счетное число связных подгрупп $H \subset G$ таких, что $\Delta$ является решеткой в $H$ и замькание по Зарисскому группы $\mathrm{Ad}(H)$ совпадает с замыканием по Зарисскому $\mathscr{A}$ групшы $\mathrm{Ad}(\Delta)$. По хорошо известному свойству алгебраических групп последнее условие влечет $[\mathscr{A}, \mathscr{A}] \subset \mathrm{Ad}(H)$. Кроме того, поскольку $\Delta-$ 
решетка в $H$, то факторпространство $\overline{[\mathscr{A}, \mathscr{A}] \operatorname{Ad}(\Delta)} \backslash \operatorname{Ad}(H)$ компактно, и теперь утверждение теоремы следует из счетности числа связных подгруп в абелевой групе $[\mathscr{A}, \mathscr{A}] \backslash \mathscr{A}$, содержаших $\overline{[\mathscr{A}, \mathscr{A}] \operatorname{Ad}(\Delta)}$ как равномерную подгруппу.

Имея в распоряжении лемму 8.4 и теорему 8.5 , нам достаточно рассмотреть поведение унипотентных траекторий вблизи сингулярных подмножеств $\Gamma X\left(H, u_{\mathbb{R}}\right) \subset \Gamma \backslash G$, $H \in \mathscr{H}$. С каждым аналитическим подмножеством $X\left(H, u_{\mathbb{R}}\right)$ оказывается возможным ассоциировать некоторое афффинное многообразие $A=A_{H}$ в векторном пространстве $V=V_{H}$, причем участку произвольной Аd-унипотентной траектории вблизи $\Gamma X\left(H, u_{\mathbb{R}}\right) \subset \Gamma \backslash G$ будет соответствовать счетньй набор участков траекторий некоторой унипотентной подгрупш из $G L(V)$ вблизи $A \subset V$. Напомним, что любое аффинное многообразие в векторном пространстве $V$ над полем $\mathbb{R}$ выделяется как множество нулей некоторого полинома. В свою очередь, координаты унипотентных траекторий также записьваются полиномами от $t$. Это позволяет доказать, используя некоторые стандартные свойства полиномов, следуюший результат о поведении унипотентных траекторий вблизи аффинных многообразий в векторном пространстве.

ЛЕмма 8.6. Пусть $A$ - аффинное многообразие в вещественном векторном пространстве $V$. Тогда для любого компакта $C \subset A$ и любого $\varepsilon>0$ найдется компакт $D \subset A$ (содержащий $C$ ) такой, что для любой окрестности $\Phi=\Phi(D) \subset V$ найдется настолько малая окрестность $\Psi=\Psi(C) \subset \Phi$, что для любой унипотентной подгруппы $\left\{u_{t}\right\} \subset G L(V)$, любой точки $v \in V-\Phi$ и любого T>0 виполняется

$$
l\left\{t \in[0, T]: u_{t} v \in \Psi\right\}<\varepsilon l\left\{t \in[0, T]: u_{t} v \in \Phi\right\} .
$$

Если для компактов $C \subset D \subset V$ вьполняется свойство, указанное в лемме, говорят, что относительный размер компакта $C$ в $D$ не превосходит $\varepsilon$.

3. Рассмотрим вначале, как доказательство теоремы 8.2 сводится к лемме 8.6 , на простейшем примере $G=S L(2, \mathbb{R}), U=\left\{u_{t}=\left(\begin{array}{cc}1 & t \\ 0 & 1\end{array}\right), t \in \mathbb{R}\right\}$. Мы предположим, что $\Gamma$ не является равномерной решеткой в $G$ (иначе сингулярное множество пусто и доказывать нечего). Ясно, что тогда $\mathscr{H}=\left\{H_{i}=g_{i} U g_{i}^{-1}, i=1,2, \ldots\right\}$ для некоторого счетного семейства элементов $\left\{g_{i}\right\}$ из $G$. Поэтому $X\left(H_{i}, U\right)=g_{i} N(U)$, где $N(U)-$ нормализатор $U$ в $G$ (очевидно, что $N(U)$ есть произведение двумерной связной подгрупшы $B$ верхнетреугольных матриц на центр $\mathbb{Z}_{2} \subset G$ ). Следовательно, сингулярное множество $Y$ всех периодических орициклов в $\Gamma \backslash G$ представляется как объединение $Y=\bigcup_{i} \Gamma g_{i} N(U), i=1,2, \ldots$ Любое множество $\Gamma g_{i} N(U)$ имеет одну (если $\mathbb{Z}_{2} \subset \Gamma$ ) либо две (если $\left.\mathbb{Z}_{2} \not \subset \Gamma\right)$ компоненты связности, причем каждая из них является иммерсированньм цилиндром без самопересечений (которьй всюду плотен в $\Gamma \backslash G$, если Г является неравномерной решеткой в $G)$.

Рассмотрим алгебру Ли $s l(2, \mathbb{R})$ как векторное пространство $V$ с базисом

$$
e_{1}=\left(\begin{array}{ll}
0 & 1 \\
0 & 0
\end{array}\right), \quad e_{2}=\left(\begin{array}{cc}
1 & 0 \\
0 & -1
\end{array}\right), \quad e_{3}=\left(\begin{array}{ll}
0 & 0 \\
1 & 0
\end{array}\right) .
$$

Для простоты мы предположим, что сама подгрупа $U$ пересекает $\Gamma$ по решетке (т.е. $U \in \mathscr{H})$, и рассмотрим множество $X(U, U)=N(U)=\mathbb{Z}_{2} \times B$. Пусть $\rho=$ Аd есть 
присоединенное представление $G$ в $V, p=e_{1} \in V$ и $\eta(g)=\rho(g) p$. Тогда орбита $\eta(G)=\rho(G) p$ есть та половина конуса $x_{1} x_{3}+x_{2}^{2}=0$, которая выделяется неравенством $x_{1}>0$. Множество $\eta(X(U, U))$ есть полупрямая $x_{2}=x_{3}=0, x_{1}>0$. Ясно, что замыкание по Зарисскому этой полупрямой есть прямая $A=\left\{x_{2}=x_{3}=0\right\}$ и $X(U, U)=\eta^{-1}(A)=\{g \in G: \eta(g) \in A\}$. Ввиду того, что $\Gamma_{U}=\Gamma \cap\left(\mathbb{Z}_{2} \times U\right)$ является равномерной решеткой в стабилизаторе $\mathbb{Z}_{2} \times U$ точки $p \in V$, образ $\eta(\Gamma)$ дискретен в $\eta(G)$. По этой же причине для любого компакта $D \subset A$ множество $\Gamma \eta^{-1}(D) \subset \Gamma \backslash G$ компактно и поэтому найдется (достаточно малая) окрестность $\Phi=\Phi(D) \subset V$, для которой множество $\Gamma \eta^{-1}(\Phi) \subset \Gamma \backslash G$ не имеет самопересечений, т.е. отображение $\Gamma g \rightarrow \eta(g), g \in \eta^{-1}(\Phi)$ корректно определено.

Пусть теперь $x=\Gamma g \notin Y$. Наша цель - для каждого $\varepsilon>0$ построить такую окрестность $\Omega$ “цилиндра" $\Gamma X(U, U) \subset \Gamma \backslash G$, что

$$
l\left\{t \in[0, T]: x u_{t} \in \Omega\right\} \leqslant \varepsilon T \text { для всех } T>0 .
$$

Так как замкнутое множество $X(U, U) \sigma$-компактно, то достаточно научиться для любого $\varepsilon>0$ и любого компакта $C \subset X(U, U)$ строить нужную окрестность $\Omega$ множества $\Gamma C \subset \Gamma \backslash G$, а затем воспользоваться приемом счетности $\varepsilon=\sum_{i=1}^{\infty} \varepsilon / 2^{i}$. Пусть $C$ - произвольньй компакт в $X(U, U), D \subset A$ - компакт, содержаший $\eta(C)$ в качестве подкомпакта относительного размера $\leqslant \varepsilon$, и $\Phi=\Phi(D) \subset V$ - та его окрестность, для которой отображение $\Gamma \eta^{-1}(\Phi) \rightarrow \Phi$ корректно определено. Рассмотрим малую окрестность $\Psi=\Psi(\eta(C)) \subset \Phi$, которая фигурирует в лемме 8.6. Докажем, что $\Omega=\Gamma \eta^{-1}(\Psi)$ является искомой окрестностью.

Зафиксируем $T>0$ и для каждого $q \in \eta(\Gamma)=\rho(\Gamma) p$ определим

$$
I(q)=\left\{t \in(0, T): \rho\left(g u_{t}\right) q \in \Phi\right\}, \quad J(q)=\left\{t \in(0, T): \rho\left(g u_{t}\right) q \in \Psi\right\} .
$$

Тогда $J(q) \subset I(q)$ и множества $I\left(q_{1}\right), I\left(q_{2}\right)$ дизъюнктны для $q_{1} \neq q_{2}$ ввиду выбора $\Phi$. Так как $\Gamma g u_{t} \in \Omega \Longleftrightarrow \exists \gamma \in \Gamma: \eta\left(\gamma g u_{t}\right) \in \Psi$, то достаточно установить неравенство $l\left(\bigcup_{q \in \eta(\Gamma)} J(q)\right) \leqslant \varepsilon T$. Пусть $(a, b)$ - любая связная компонента $I(q)$. Тогда, положив в лемме $8.6 v=\rho\left(g u_{a}\right) q$ и $T=b-a$, мы получим $l(J(q) \cap(a, b)) \leqslant \varepsilon(b-a)$ и поэтому $l(J(q)) \leqslant \varepsilon l(I(q))$ для всех $q \in \eta(\Gamma)$. Теперь наше неравенство следует из дизъюнктности множеств $I(q)$. Наконец, рассмотрев аналогично остальные "цилиндры" $\Gamma g_{i} X(U, U)$ и снова воспользовавшись приемом счетности, мы построим нужную окрестность сингулярного множества $Y \subset \Gamma \backslash G$.

4. Приступим к рассмотрению общего случая. Пусть $G$ - произвольная связная группа Ли. С каждой подгруппой $H \in \mathscr{H}$ размерности $h$ мы свяжем векторное пространство $V=V_{H}=\wedge^{h} \mathfrak{g}$ - внешнюю $h$-степень алгебры Ли $\mathfrak{g}$ и представление $\rho=$ $\rho_{H}=\wedge^{h} \mathrm{Ad}: G \rightarrow G L\left(V_{H}\right)$ - внешнюю $h$-степень присоединенного представления. Заметим, что $\rho\left(g g^{\prime}\right)=\rho\left(g^{\prime}\right) \rho(g)$ (так как $\mathrm{Ad}_{g}$ является дифференщиалом сопряжения $h \rightarrow g^{-1} h g$ ). Пусть $\xi_{1}, \ldots, \xi_{h}$ - базис подалгебры $\mathfrak{h} \subset \mathfrak{g}$ подгрупшы $H \subset G$. Тогда $\wedge^{h} \mathfrak{h}=\mathbb{R} p$, где $p=p_{H}=\xi_{1} \wedge \cdots \wedge \xi_{h} \in V$. Также обозначим через $\eta=\eta_{H}: G \rightarrow V$ отображение $\eta(g)=\rho(g) p, g \in G$. Ясно, что $\eta(H) \subset \mathbb{R} p$. Наконец, для $H \in \mathscr{H}$ пусть $\Gamma_{H}=N_{\Gamma}(H)$ - нормализатор $H$ в $\Gamma$.

Лемма 8.7. Если $H \in \mathscr{H}, m o \Gamma_{H}=\{\gamma \in \Gamma: \rho(\gamma) p= \pm p\}$. 
ДокАЗАТЕльСтво. Если $\rho(\gamma) p= \pm p$ для $\gamma \in \Gamma$, то $\operatorname{Ad}(\gamma)$ нормализует $\mathfrak{h}$ и, следовательно, $\gamma \in \Gamma_{H}$. Обратно, если $\gamma \in \Gamma_{H}$, то $\gamma$ нормализует решетку $\Gamma \cap H \subset H$ и поэтому сопряжение $h \rightarrow \gamma^{-1} h \gamma$ сохраняет биинвариантную меру Хаара на $H$ и (с точностью до знака) форму объема на $\mathfrak{h}$.

Лемма 8.8. Пусть $H \in \mathscr{H}$ и $A=A_{H} \subset V$ есть замыкание по Зарисскому множества $\eta\left(X\left(H, u_{\mathbb{R}}\right)\right)$. Тогда $X\left(H, u_{\mathbb{R}}\right)=\eta^{-1}(A)=\{g \in G: \eta(g) \in A\}$.

ДокаЗАтельство. Ясно, что $X\left(H, u_{\mathbb{R}}\right) \subset \eta^{-1}(A)$. Обратно, пусть $\mathfrak{u} \subset \mathfrak{g}$ есть (одномерная) подалгебра Ли для $u_{\mathbb{R}} \subset G$. Тогда $g \in X\left(H, u_{\mathbb{R}}\right) \Longleftrightarrow \mathfrak{u} \subset \operatorname{Ad}_{g}(\mathfrak{h}) \Longleftrightarrow$ $\eta(g) \wedge \mathfrak{u} \equiv 0 \Longleftrightarrow \eta(g) \in A_{0}$, где $A_{0}=\{v \in V: v \wedge \mathfrak{u} \equiv 0\}$ замкнуто по Зарисскому и потому содержит $A$.

Теперь мы рассмотрим строение сингулярных подмножеств $\Gamma X\left(H, u_{\mathbb{R}}\right) \subset \Gamma \backslash G$ для $H \in \mathscr{H}$. Если каждое такое множество не имеет самопересечений, то доказательство для $G=S L(2, \mathbb{R})$ проходит практически без изменений в общем случае. Однако если оно имеет самопересечения, доказательство усложняется, поскольку оказывается невозможным построить корректно определенное отображение какой-либо окрестности $\eta^{-1}(D) \subset \Gamma \backslash G$ на окрестность компакта $D \subset A$. Впервые это затруднение было преодолено Шахом [Sh91].

ОПРЕДЕЛЕНИЕ 8.9. Пусть $M-$ подмножество в $G$. Скажем, что $g \in M$ есть точка $(H, \Gamma)$-самопересечения, если найдется такой элемент $\gamma \in \Gamma-\Gamma_{H}$, что $\gamma g \in M$.

Эквивалентно, $g \in M$ не есть точка $(H, \Gamma)$-самопересечения, если из $\Gamma g=\Gamma g^{\prime}$, $g^{\prime} \in M$, следует $\Gamma_{H} g=\Gamma_{H} g^{\prime}$.

Нас будут интересовать самопересечения множества $X\left(H, u_{\mathbb{R}}\right)$. Предположим, что $u_{\mathbb{R}} \subset H$. Тогда $N(H) \subset X\left(H, u_{\mathbb{R}}\right)$ и если $N(H)=X\left(H, u_{\mathbb{R}}\right)$, то $(H, \Gamma)$-самопересечений у $X\left(H, u_{\mathbb{R}}\right)$ нет. Однако в обшем случае имеет место строгое включение и возможны самопересечения.

ПримеР 8.10. Пусть $G=S L(2, \mathbb{C}), \Gamma=S L(2, \mathbb{Z}+i \mathbb{Z}), u_{t}=\left(\begin{array}{l}1 t \\ 0 \\ 0\end{array}\right)$ и $H=S L(2, \mathbb{R})$. Тогда $X\left(H, u_{\mathbb{R}}\right)=H u_{\mathbb{R}}^{\prime}$, где $u_{t}^{\prime}=\left(\begin{array}{cc}1 & i t \\ 0 & 1\end{array}\right)$ и $\Gamma_{H}=S L(2, \mathbb{Z})$. Легко проверить, что множество точек $(H, \Gamma)$-самопересечения $X\left(H, u_{\mathbb{R}}\right)$ есть $u_{\mathbb{R}} \times u_{\mathbb{R}}^{\prime}$ (иначе говоря, множество $\Gamma X\left(H, u_{\mathbb{R}}\right) \subset \Gamma \backslash G$ самопересекается по тору $\left.\Gamma\left(u_{\mathbb{R}} \times u_{\mathbb{R}}^{\prime}\right)\right)$.

K счастью, бороться с самопересечениями позволяют индукция по размерности и следующее утверждение.

Лемма 8.11. Пусть $H \in \mathscr{H} u h=\operatorname{dim}(H)$. Тогда множество точек $(H, \Gamma)$-самопересечений $X\left(H, u_{\mathbb{R}}\right)$ лежит в обвединении всех $X\left(H^{\prime}, u_{\mathbb{R}}\right)$, где $H^{\prime} \in \mathscr{H} u \operatorname{dim}\left(H^{\prime}\right) \leqslant h-1$.

ДокАЗАТельство. Пусть $g \in X\left(H, u_{\mathbb{R}}\right)$ есть точка $(H, \Gamma)$-самопересечения. Тогда найдется элемент $\gamma \in \Gamma-\Gamma_{H}$ такой, что $g u_{\mathbb{R}} g^{-1} \subset H \cap \gamma^{-1} H \gamma$. Так как $\gamma \notin \Gamma_{H}$, то $\operatorname{dim}\left(H \cap \gamma^{-1} H \gamma\right) \leqslant h-1$. Подпространство $\Gamma\left(H \cap \gamma^{-1} H \gamma\right)$ открыто и замкнуто в пересечении двух замкнутых подпространств $Г H$ и $Г H \gamma$ (это следует из дискретности $\Gamma \subset G)$. Следовательно, оно само является замкнутым. Заметим, что пересечение двух однородных подпространств конечного объема, вообще говоря, не обязано иметь 
конечньй объем (например, ГН и $Г k H k^{-1}$, где $k=\left(\begin{array}{cc}i & 0 \\ 0 & -i\end{array}\right)$, из примера 8.10 пересекаются по одномерному некомпактному многообразию). Эту трудность мы преодолеем следуюшим образом. Пусть $H_{\gamma}$ - наименьшая (связная) подгруппа в $H \cap \gamma^{-1} H \gamma$, содержашая все унипотентные в $G$ однопараметрические подгрупшы. Тогда $H_{\gamma}$ нормальна в $H \cap \gamma^{-1} H \gamma$ и поэтому $H_{\gamma}$ действует эргодически на $\left(\Gamma \cap H^{\prime}\right) \backslash H^{\prime}$, где $H^{\prime}=\left(\overline{\Gamma H_{\gamma}}\right)_{0}$, относительно локально конечной $H^{\prime}$-инвариантной меры. В силу D.10 эта мера конечна и поэтому $g \in X\left(H^{\prime}, u_{\mathbb{R}}\right)$, где $H^{\prime} \in \mathscr{H}$ и $\operatorname{dim}\left(H^{\prime}\right) \leqslant h-1$.

Теперь мы приведем без доказательства один из основных результатов [DM93].

ТеОрема 8.12. Пусть $\Gamma-$-искретная подгруппа в $G$ и $H \in \mathscr{H}$. Тогда орбита $\eta(\Gamma)$ дискретна в $\mathrm{V}$.

Для иллюстрации этой теоремы рассмотрим тот важный случай, когда $G$ - замкнутая подгруппа в $S L(n, \mathbb{R})$ и $\Gamma \subset G \cap S L(n, \mathbb{Z})$. В этом случае связной подгрупше $H$, пересекаюшей $\Gamma$ по решетке, отвечает рациональная подалгебра $\mathfrak{h} \subset M(n, \mathbb{R})$. Поэтому вектор $p \in V$ коллинеарен вектору с рациональньми координатами, в то время как $\rho(\Gamma)$ состоит из целочисленных матриц. Следовательно, орбита $\eta(\Gamma)=\rho(\Gamma) p$ дискретна.

СлЕДСТВИЕ 8.13. Пусть $H \in \mathscr{H}, A$ - замыкание по Зарисскому $\eta\left(X\left(H, u_{\mathbb{R}}\right)\right)$ в $V$ и $D$ - компакт в $A$. Обозначим через $Y_{D}$ множсество точек $(H, \Gamma)$-самопересечения $\eta^{-1}(D)$ и пусть $K$ - компакт в $G-\Gamma Y_{D}$. Тогда существует окрестность $\Phi$ компакта $D$ в $V$ такая, что $\eta^{-1}(\Phi) \cap \Gamma K$ не имеет точек самопересечения.

ДокАЗАТЕльство. Предположим, напротив, что найдутся последовательности $\left\{g_{i}\right\},\left\{g_{i}^{\prime}\right\}$ элементов из $G$ таких, что $\Gamma g_{i}=\Gamma g_{i}^{\prime} \in \Gamma K, \Gamma_{H} g_{i} \neq \Gamma_{H} g_{i}^{\prime}$ и $\eta\left(g_{i}\right) \rightarrow d \in D$, $\eta\left(g_{i}^{\prime}\right) \rightarrow d^{\prime} \in D$. Ввиду компактности $K$ можно считать, что $g_{i}=\gamma_{i} h_{i}, g_{i}^{\prime}=\gamma_{i}^{\prime} h_{i}$, где $\gamma_{i}, \gamma_{i}^{\prime} \in \Gamma$ и $h_{i} \rightarrow h \in K$. Тогда $\eta\left(\gamma_{i}\right)=\rho\left(h_{i}^{-1}\right) \eta\left(g_{i}\right) \rightarrow \rho\left(h^{-1}\right) d$ и, аналогично, $\eta\left(\gamma_{i}^{\prime}\right) \rightarrow \rho\left(h^{-1}\right) d^{\prime}$. Из дискретности множества $\eta(\Gamma)$ следует $\eta\left(\gamma_{i}\right)=\rho\left(h^{-1}\right) d$, $\eta\left(\gamma_{i}^{\prime}\right)=\rho\left(h^{-1}\right) d^{\prime}$, т.е. $\gamma_{i} h, \gamma_{i}^{\prime} h \in \eta^{-1}(D) \subset X\left(H, u_{\mathbb{R}}\right)$ для больших $i$. Так как $\Gamma h \in \Gamma K$ и $\Gamma K \cap Y_{D}=\varnothing$, то никакой элемент вида $\gamma_{i} h$ не является точкой $(H, \Gamma)$-самопересечения. Поэтому из $\Gamma \gamma_{i} h=\Gamma \gamma_{i}^{\prime} h$ вытекает $\Gamma_{H} \gamma_{i}=\Gamma_{H} \gamma_{i}^{\prime}$, и тогда $\Gamma_{H} g_{i}=\Gamma_{H} \gamma_{i} h_{i}=$ $\Gamma_{H} \gamma_{i}^{\prime} h_{i}=\Gamma_{H} g_{i}^{\prime}$. Противоречие.

ДОКАЗАТЕЛЬСТВО ТЕОРЕМЫ 8.2. Используя структуру сингулярного множества $Y \subset X$, достаточно для любых $x \in X-Y, H \in \mathscr{H}, \varepsilon>0$ и любых компактов $C \subset$ $X\left(H, u_{\mathbb{R}}\right), K \subset \Gamma \backslash G$ научиться строить такую окрестность $\Omega$ компакта $\Gamma C \subset \Gamma \backslash G$, что

$$
l\left\{t \in[0, T]: x u_{t} \in \Omega \cap K\right\} \leqslant \varepsilon T \text { для всех } T \geqslant 0 .
$$

Мы проделаем это, используя индукцию по $\operatorname{dim}(H)$. Для $\operatorname{dim}(H)=1$ утверждение тривиально. Предположим, что оно доказано для $\operatorname{dim}(H) \leqslant n-1$. Зафиксируем $x \in X-Y, H \in \mathscr{H}, \varepsilon>0, C \subset X\left(H, u_{\mathbb{R}}\right)$ и $K \subset \Gamma \backslash G$. Пусть $Y_{H} \subset G$ есть множество точек $(H, \Gamma)$-самопересечения множества $X\left(H, u_{\mathbb{R}}\right)$. Тогда по лемме 8.11 и по предположению индукции найдется окрестность $\Omega^{\prime}$ множества $\Gamma Y_{H} \subset \Gamma \backslash G$ такая, что

$$
l\left\{t \in[0, T]: x u_{t} \in \Omega^{\prime} \cap K\right\} \leqslant \varepsilon T / 2 \text { для всех } T \geqslant 0 .
$$


Пусть, далее, $V=V_{H}, \rho=\rho_{H}, p=p_{H}, \eta=\eta_{H}$ и $A=A_{H}$ есть замыкание по Зарисскому множества $\eta\left(X\left(H, u_{\mathbb{R}}\right)\right)$. По лемме 8.6 найдется компакт $D \subset A$, содержаший $\eta(C)$ в качестве подкомпакта относительного размера не более $\varepsilon / 4$. Зафиксируем $\Gamma g=x \in X-Y$. Так как образ $\eta(\Gamma) \subset V$ дискретен, то множество $\eta(\Gamma g)=\rho(g) \eta(\Gamma)$ дискретно, причем $\eta(\Gamma g) \cap A=\varnothing$. Пусть $K^{\prime}$ - такой компакт в $G$, что $\Gamma K^{\prime}=K_{1}=K-\Omega^{\prime} \subset \Gamma \backslash G$. Используя 8.13 , нетрудно найти окрестность $\Phi=\Phi(D)$ такую, что $\Phi \cap \eta(\Gamma g)=\varnothing$ и множество $\eta^{-1}(\Phi) \cap \Gamma K^{\prime}$ не имеет $(H, \Gamma)$-самопересечений.

Рассмотрим теперь окрестность $\Psi=\Psi(\eta(C)) \subset \Phi \subset V$, для которой

$l\left\{t \in[0, T]: \rho\left(u_{t}\right) v \in \Psi\right\}<\varepsilon l\left\{t \in[0, T]: \rho\left(u_{t}\right) v \in \Phi\right\} / 4$ для всех $v \in V-\Phi, \quad T>0$.

Теперь уже по аналогии со случаем $G=S L(2, \mathbb{R})$ нетрудно доказать, что окрестность $\Omega=\Gamma \eta^{-1}(\Psi)$ компакта $\Gamma C \subset \Gamma \backslash G$ является искомой. Тем самым мы доказали теоремы 8.2, 8.3 и 8.1 (в частности, топологическую гипотезу Рагунатана для однопараметрических подгруп).

5. Обсудим теперь топологическую гипотезу Рагунатана для произвольных подгруп $U \subset G$, порожденных унипотентными элементами (т.е. в обобщенной форме, предложенной Маргулисом). Марина Ратнер полностью исследовала случай, когда $U$ имеет вид как в теореме 7.1. Следуя [R91b], мы докажем алгебраичность замьканий $U$-орбит в ситуации, когда $U$ связна и целиком состоит из унипотентных элементов (общий случай требует более тонких рассуждений). Зафиксируем $x=\Gamma g \in \Gamma \backslash G$. Мы знаем, что для каждой подгруппы $u_{\mathbb{R}} \subset U$ найдется такая связная подгруппа $H \subset G$, что $\overline{x u_{\mathbb{R}}}=x H$ и $x H$ имеет конечную $H$-инвариантную $u_{\mathbb{R}}$-эргодическую меру. По теореме D.4 решетка $\Gamma \cap g H^{-1}$ плотна по Зарисскому в $g H^{-1}$ относительно Ad-представления групшы $G$. Таким образом, семейство связных подгрупп $H \subset G$ таких, что $x H$ имеет конечньй объем и $\overline{x u_{\mathbb{R}}}=x H$ для некоторой подгруппы $u_{\mathbb{R}} \subset U$, по теореме 8.5 не более чем счетно. Следовательно, связная подгруппа $U$ содержится в счетном объединении подгруп $H_{i} \subset G$. Из соображений размерности найдется $H_{i} \subset G$ такая, что $U \subset H_{i}$, и тогда, очевидно, $\overline{x U}=x H_{i}$.

Недавно обобщенная гипотеза Рагунатана была "почти полностью” доказана Шахом.

TeOPEмa 8.14 ([Sh97]). Пусть пространство $\Gamma \backslash G$ имеет конечный обвем и подгруппа $U \subset G$ порождена унипотентними әлементами. Тогда для любой точки $x \in \Gamma \backslash G$ найдется подгруппа $F \subset G$ такая, что $\overline{x U}=x F$ и $x F_{0}$ имеет конечную $F_{0}$-инвариантную меру.

То, что и $x F$ имеет конечную $F$-инвариантную меру, показано Эскиным и Маргулисом (устное сообщение). Таким образом, обобшенную гипотезу Рагунатана можно считать полностью доказанной. В частности, из результатов Шаха, Эскина и Маргулиса вытекает решение следуюшего вопроса, упомянутого в [R94]. Пусть $G$ - простая вешественная группа Ли и $U \subset G$ - ее неравномерная решетка (она заведомо порождена унипотентными элементами). Тогда каждая $U$-орбита на пространстве $\Gamma \backslash G$ конечного объема имеет алгебраическое замыкание. В частности, если произведение двух решеток в $G$ замкнуто, то они соизмеримы. Заметим, что для $G=\operatorname{PS} L(2, \mathbb{C})$ ранее это было доказано Шахом. 
6. Напоследок покажем, как гипотеза Рагунатана сводится к случаю решетки $\Gamma \subset G$ (именно в таком виде были получены результаты Ратнер). Пусть $G=L \cdot R$ - разложение Леви связной односвязной групшы Ли $G$. Рассмотрим группу $A(G)=\left(L \times A\left(T_{R}\right)\right) \cdot R$, введенную в конце $\S 7$. Как было сказано, на $A(G)$ можно имитировать структуру вешественно-алгебраической группы. В [St89] доказано, что если $\Gamma \backslash G$ имеет конечный объем, то $\Gamma$ “почти плотна по Зарисскому" в $G$. Точнее, $A(\Gamma)$ нормальна в $A(G)$ и найдется тор $T \subset A\left(T_{\mathbb{R}}\right)$ такой, что $A(G)=(K \times T) A(\Gamma)$, где $K$ - компактная компонента подгруппы Леви $L \subset G$. Не ограничивая обшности мы можем предположить, что $K$ не имеет центральных в $G$ подгрупп. Тогда $A(\Gamma)$ содержит все унипотентные элементы и поэтому $U \subset A(\Gamma)$. Теперь при рассмотрении орбиты $\Gamma U$ мы можем сосредоточить внимание на подпространстве конечного объема $\Gamma \backslash H$, где $H=G \cap A(\Gamma)$. При этом связная компонента $\Gamma_{0}$ нормальна в $A(\Gamma)$ и, стало быть, в $H$. Следовательно, мы можем воспользоваться теоремой 8.14 для решетки $\Gamma_{0} \backslash(\Gamma \cap H)$ в групше Ли $\Gamma_{0} \backslash H$ (возможная несвязность $H$ в данном случае не приводит к затруднениям).

\section{$\S$ 9. Различные вопросы сходимости мер}

Здесь мы приводим без доказательства обобшения теоремы 8.1 о равномерной распределенности унипотентных орбит; см. также [R94,95b]. Все они опираются на метрическую теорему Ратнер 7.1, но саму теорему 8.1 не используют.

1. Точка $x \in \Gamma \backslash G$, подгруппа $u_{\mathbb{R}} \subset G$ и число $T>0$ определяют вероятностную меру $\mu_{T, x, u}$ на $\Gamma \backslash G$ по правилу

$$
\mu_{T, x, u}(f)=\frac{1}{T} \int_{0}^{T} f\left(x u_{t}\right) d t, \quad f \in C_{c}(\Gamma \backslash G)
$$

(т.е. $\mu_{T, x, u}(f)$ есть временное среднее функции $f$ вдоль куска траектории $\left.x u_{[0, T]}\right)$. Напомним, что сингулярное множество $Y=Y\left(u_{\mathbb{R}}\right) \subset \Gamma \backslash G$ для унипотентного потока $\left(\Gamma \backslash G, u_{\mathbb{R}}\right)$ (т.е. объединение всех собственных инвариантных замкнутых однородных подпространств) является счетным объединением локально аналитических множеств: $Y=\bigcup_{H \in \mathscr{H}} \Gamma X\left(H, u_{\mathbb{R}}\right)$. Теорема 8.1 в точности означает, что $\mu_{T, x, u}$ в $*$-слабой топологии стремится к $G$-инвариантной мере $\nu$ при $T \rightarrow \infty$ для любой точки $x$ вне сингулярного множества $Y$.

Заметим, что для орициклического потока $\left(\Gamma \backslash S L(2, \mathbb{R}), u_{\mathbb{R}}\right)$ с равномерной решеткой $\Gamma$ каждая траектория равномерно распределена в $\Gamma \backslash S L(2, \mathbb{R}$ ) (и это эквивалентно строгой эргодичности). Довольно тонкие оценки для асимптотики сходимости $\mu_{T, x, u} \rightarrow \nu$ в этом случае найдены Бургером $[\mathrm{Bu}]$.

Оказьвается, сходимость имеет место и для последовательности точек $x_{n} \in \Gamma \backslash G$, сходящейся к несингулярной точке $x \in \Gamma \backslash G$. Первая часть следующей теоремы была независимо также доказана Ратнер [R94].

Tеорема 9.1 ([DM93]). а) Пусть $T_{n} \rightarrow+\infty u x_{n} \rightarrow x$, причем $x \in \Gamma \backslash G$ является несингулярной для унипотентной подгруппь $u_{\mathbb{R}} \subset G$. Тогда $\mu_{T_{n}, x_{n}, u} \rightarrow \nu$, $n \rightarrow \infty$.

b) Более того, для функиии $f \in C_{c}(\Gamma \backslash G)$, компакта $K \subset \Gamma \backslash G$ и числа $\varepsilon>0$ найдется конечное число подгрупп $H_{i} \in \mathscr{H}, i=1, \ldots, k$, и компактов $C_{i} \subset$ 
$X\left(H_{i}, u_{\mathbb{R}}\right)$ таких, что $\left|\mu_{T, x, u}(f)-\nu(f)\right|<\varepsilon$ равномерно по любому подкомпакту $F \subset K-\bigcup_{i} \Gamma C_{i}$ для всех $T \geqslant T_{0}=T_{0}(F)$.

Для иллюстрации рассмотрим любую последовательность периодических орициклов $x_{n} u_{\mathbb{R}} \subset \Gamma \backslash S L(2, \mathbb{R})$ с периодами $T_{n} \rightarrow \infty$ и соответствуюшими эргодическими мерами $\mu_{T_{n}}$. Нетрудно доказать (используя свойство топологического перемешивания геодезического потока), что для сколь угодно малой окрестности $O_{\varepsilon}(x)$ произвольной точки $x \in \Gamma \backslash S L(2, \mathbb{R})$ найдется $N$ такое, что $x_{n} u_{\mathbb{R}} \cap O_{\varepsilon}(x) \neq \varnothing$ для всех $n \geqslant N$. Следовательно, найдутся точки $x_{n}^{\prime} \in x_{n} u_{\mathbb{R}}$, сходящиеся к $x$. Применяя теперь предыдущий результат, мы получаем теорему Сарнака [Sa] о равномерной распределенности: периодический орищикл $x_{n} u_{\mathbb{R}}=x_{n}^{\prime} u_{\mathbb{R}}$ становится все более и более равномерно распределенньм в $\Gamma \backslash S L(2, \mathbb{R})$ при $T_{n} \rightarrow \infty$ (элементарное доказательство этого факта можно также найти у Ратнер в [R92]).

На самом деле Сарнак нашел и асимптотику сходимости $\mu_{T} \rightarrow \nu$ с точностью до $o\left(T^{-1 / 2}\right)$ для всех неравномерных решеток $\Gamma \subset G$. Для $\Gamma=S L(2, \mathbb{Z})$ она принимает особенно простой вид (найденньй впервые Загьером $[\mathrm{Za}]): \mu_{T}(f)=\nu(f)+o\left(T^{-1 / 2}\right)$, $T \rightarrow+\infty$, для каждой $f \in C_{c}^{\infty}(\Gamma \backslash S L(2, \mathbb{R}))$. В [Za] найдена и замечательная связь между скоростью сходимости $\mu_{T}(f) \rightarrow \nu(f), T \rightarrow+\infty$, и знаменитой гипотезой Римана из теории чисел: она верна тогда и только тогда, когда $\mu_{T}(f)=\nu(f)+$ $o\left(T^{-3 / 4+\varepsilon}\right)$ для каждого $\varepsilon>0$ и каждой $f \in C_{c}^{\infty}(\Gamma \backslash S L(2, \mathbb{R}))$. Элементарное доказательство результата Загьера и подробное изучение арифметических свойств орбифолда $S L(2, \mathbb{Z}) \backslash S L(2, \mathbb{R})$ можно найти в работе Веревского [Ve]. Там же доказано, что $\alpha=-1 / 2$ является оптимальной константой, если пытаться найти асимптотику вида $o\left(T^{\alpha}\right)$ для характеристических функций простейших открытых подмножеств в $S L(2, \mathbb{Z}) \backslash S L(2, \mathbb{R})$ (типа кубической окрестности). Разумеется, это не опровергает гипотезу Римана, так как такие функции разрьвны.

2. Результат о равномерной распределенности замкнутых орбит можно доказать и для действия полупростых подгрупп $H \subset G$.

ТЕОРема 9.2 ([DM93]). Пусть $H \subset G$ - максимальная связная собственная подгруппа, причем $H$ полупроста и не имеет компактных множителей. Предположим, что каждая орбита $x_{n} H$ замкнута и поэтому несет на себе единственную $H$-инвариантную вероятностную меру $\mu_{n}$. Пусть $x_{n} \rightarrow x, n \rightarrow \infty$, причем орбита $x H$ не замкнута. Тогда $\mu_{n} \rightarrow \nu, n \rightarrow \infty$.

ДокАЗАТЕЛЬСтво. Ясно, что $H$ содержит унипотентную подгрупу $u_{\mathbb{R}}$, которая не содержится в собственной нормальной в $H$ подгрупше. Тогда по критерию эргодичности Мура С.1 каждый поток $\left(x_{n} H, u_{\mathbb{R}}\right)$ эргодичен. Пусть $x=\Gamma g$ для некоторого $g \in G$. Докажем, что точка $\Gamma g h \in \Gamma \backslash G$ не является сингулярной (в смысле § 8) для потока $\left(\Gamma \backslash G, u_{\mathbb{R}}\right)$ для почти каждого $h \in H$. Если это не так, то, используя структуру сингулярного множества, мы найдем собственную подгруппу $L \subset G$ такую, что $\Gamma \cap L$ - решетка в $L$ и $g h \in X\left(L, u_{\mathbb{R}}\right)=\left\{f \in G: f u_{\mathbb{R}} f^{-1} \subset L\right\}$ для множества $h \in H$ положительной меры. Так как множество $X\left(L, u_{\mathbb{R}}\right)$ аналитично, то пересечение $g H \cap X\left(L, u_{\mathbb{R}}\right)$ может иметь положительную меру, лишш если $g H \subset X\left(L, u_{\mathbb{R}}\right)$, и поэтому $h u_{\mathbb{R}} h^{-1} \subset g^{-1} L g$ для всех $h \in H$. Отсюда ввиду выбора $u_{\mathbb{R}}$ и максимальности $H$ мы вьводим, что $H=g^{-1} L g$. Но тогда орбита $\Gamma g H=\Gamma L g$ замкнута, что противоречит условию на $x=\Gamma g$. 
Итак, точка $x h$ не является сингулярной для $u_{\mathbb{R}}$ при почти всех $h \in H$. Для каждого $n$ орбита $x_{n} h u_{\mathbb{R}}$ равномерно распределена ввиду эргодической теоремы Биркгофа-Хинчина для почти всех $h \in H$. Следовательно, найдется такой элемент $h \in H$, что каждая орбита $x_{n} h u_{\mathbb{R}}$ равномерно распределена в $x_{n} H$ по отношению к $\mu_{n}$ и точка $x h \in \Gamma \backslash G$ не является сингулярной для $u_{\mathbb{R}}$. Применяя теперь теорему 9.1 к последовательности $x_{n} h \rightarrow x h$, мы получаем требуемый результат.

Заметим, что доказанная теорема обобщает теорему Бургера и Сарнака [BS] о равномерной распределенности так называемых точек Гекке, возникаюших в теории чисел. Чтобы получить результат $[\mathrm{BS}]$, нужно взять некомпактную простую группу $S$ с решеткой $\Lambda$ и рассмотреть случай, когда $H$ - диагональная подгруппа в группе Ли $G=S \times S$ с решеткой $\Gamma=\Lambda \times \Lambda$.

3. Как показали Дани и Маргулис, теорему 8.1 о равномерной распределенности можно обобщить еше дальше, если варьировать не только точку $x \in \Gamma \backslash G$, но и саму подгрупшу $u_{\mathbb{R}}$ :

Теорема 9.3 ([DM93]). Рассмотрим последовательность унипотентных подгрупп $u_{\mathbb{R}}^{(n)}$, сходящуюся $\kappa$ унипотентной подгруппе $u_{\mathbb{R}}$ (это означает, что $u_{t}^{(n)} \rightarrow u_{t}$ при $n \rightarrow \infty$ для любого $\left.t \in \mathbb{R}\right)$, и такую последовательность точек $x_{n} \rightarrow x$, что предел $x \in \Gamma \backslash G$ является несингулярной точкой для $u_{\mathbb{R}} \subset G$. Тогда $\mu_{T_{n}, x_{n}, u^{(n)}} \rightarrow \nu, T_{n} \rightarrow+\infty$.

4. Пусть теперь $\Gamma$ - произвольная дискретная подгруппа в $G, X=\Gamma \backslash G$ и $\mathscr{P}(X)-$ афффинное пространство вероятностных борелевских мер на $X$ с топологией $*$-слабой сходимости. Следуя Мозесу и Шаху [MS], обозначим через $Q(X)$ подмножество всех мер $\mu \in \mathscr{P}(X)$, для которых найдется однопараметрическая унипотентная подгруппа $u_{\mathbb{R}} \subset G$ такая, что $\mu$ является инвариантной эргодической мерой для потока $\left(X, u_{\mathbb{R}}\right)$.

TeOpema 9.4 ([MS]). Множество $Q(X)$ замкнуто в $\mathscr{P}(X)$.

Это означает, что если $\mu_{n} \in Q(X)$ и $\mu_{n} \rightarrow \mu \in \mathscr{P}(X)$, то мера $\mu$ также является эргодической относительно некоторой унипотентной подгрупшы, т.е. $\mu \in Q(X)$.

Предположим теперь, что $\Gamma$ - решетка в $G, X=\Gamma \backslash G$ и $X^{c}$ - одноточечная компактификация $X$ (если $X$ некомпактно). Тогда пространство $\mathscr{P}\left(X^{c}\right)$ компактно (относительно $*$-слабой топологии). Согласно теореме D.8 фиксированная унипотентная траектория $x u_{\mathbb{R}} \subset X$ большую часть времени проводит в некотором компакте $K \subset X$. Оказывается, этот результат можно обобшить, если разрешить точкам $x$ пробегать произвольный компакт $C \subset X$, а компакт $K$ выбирать единьм для всех унипотентных подгрупп $u_{\mathbb{R}} \subset X$ :

ТЕОРема 9.5 ([DM93]). Пусть С - произвольный компакт в пространстве $\Gamma \backslash G$ конечного обгема. Тогда для любого $\varepsilon>0$ найдется компакт $K \subset \Gamma \backslash G$ (содержсащий $C$ ) такой, что

$$
l\left\{t \in[0, T]: x u_{t} \in K\right\} \geqslant(1-\varepsilon) T
$$

для всех $T>0, x \in C$ и всех унипотентных подәрупп $u_{\mathbb{R}} \subset G$.

Этот результат применяется для доказательства теоремы 9.3 и позволяет нам получить следующее 
СЛЕДСТвИЕ 9.6 ([MS]). Пусть $X=\Gamma \backslash G$ - пространство конечного оббема $u \nu_{\infty}$ - вероятностная мера на $X^{c}$, сосредоточенная на бесконечности (если $X$ некомпактно). Тогда множество $Q(X) \cup \nu_{\infty} \subset \mathscr{P}\left(X^{c}\right)$ компактно.

В самом деле, пусть $\mu_{n} \in Q(X)$ и $\mu_{n} \rightarrow \mu \in \mathscr{P}\left(X^{c}\right)$. Если $\mu \neq \nu_{\infty}$, то найдется компакт $C \subset X$ с $\mu(C)>0$. Тогда, без ограничения обшности, $\mu_{n}(C)>0$ для всех $n$. Возьмем любое $\varepsilon>0$ и компакт $K=K(\varepsilon, C)$ из теоремы 9.5. Пусть мера $\mu_{n}$ эргодична относительно унипотентной подгрупш $u_{\mathbb{R}}^{(n)} \subset G$. Из теоремы Биркгофа-Хинчина и теоремы 9.5 следует, что $\mu_{n}(K)>1-\varepsilon$ для всех $n$, и поэтому $\mu(K) \geqslant 1-\varepsilon$. Следовательно, $\mu(X)=1$, т.е. $\mu \in \mathscr{P}(X)$, и осталось применить замкнутость $Q(X)$ в $\mathscr{P}(X)$.

Примером последовательности $\left\{\mu_{n} \in Q(X)\right\}$, сходящейся к $\nu_{\infty}$, могут служить нормированные меры на последовательности все более коротких периодических орициклов в $S L(2, \mathbb{Z}) \backslash S L(2, \mathbb{R})$.

Зафиксируем точку $x \in X$ и обозначим через $Q(x)$ множество тех мер из $Q(X)$, носитель которых содержит точку $x$. Из 9.6 сразу вытекает, что $Q(x)$ - компакт.

5. В ряде проблем, связанных с теорией чисел (см. §14), приходится иметь дело со сходимостью эргодических мер для редуктивных алгебраических подгрупп (заметим, что такая подгруппа не обязана в общем случае порождаться унипотентными элементами). Общая постановка здесь такова: пусть $H \subset G \subset S L(n, \mathbb{R})$ - две редуктивные алгебраические подгруппы над $\mathbb{Q}$, причем обе не допускают нетривиальных $\mathbb{Q}$-характеров. Тогда по теореме В.4 Бореля и Хариш-Чандры $G_{\mathbb{Z}}=G \cap S L(n, \mathbb{Z})$ и $H_{\mathbb{Z}}=H \cap S L(n, \mathbb{Z})$ являются решетками. Для каждой вероятностной меры $\mu \in$ $\mathscr{P}\left(G_{\mathbb{Z}} \backslash G\right)$ через $\mu g, g \in G$, обозначим ее сдвиг: $\mu g(E)=\mu\left(E g^{-1}\right)$. Мы получили непрерьвное действие группы $G$ на пространстве $\mathscr{P}\left(G_{\mathbb{Z}} \backslash G\right)$, снабженном *-слабой топологией. Через $\mu_{H}$ и $\mu_{G}$ обозначим вероятностные $H$ - и $G$-инвариантные меры на пространствах $G_{\mathbb{Z}} H \simeq H_{\mathbb{Z}} \backslash H$ и $G_{\mathbb{Z}} \backslash G$ соответственно. Пусть $\left\{g_{i}\right\}$ - последовательность элементов из $G$. Возникает вопрос: какие меры из $\mathscr{P}\left(G_{\mathbb{Z}} \backslash G\right)$ могут быть предельными для последовательности $\left\{\mu_{H} g_{i}\right\}$ ? В частности, при каких условиях можно гарантировать, что $\mu_{H} g_{i} \rightarrow \mu_{G}$ ? Когда семейство мер $\left\{\mu_{H} g, g \in G\right\}$ является предкомпактньц?

Ответы на эти вопросы получены в работах Эскина, Мозеса и Шаха [EMS96,97]. Начнем с последнего вопроса. Через $\pi: G \rightarrow G_{\mathbb{Z}} \backslash G$ обозначим естественную проекцию. Так как $H$ является $\mathbb{Q}$-группой, то же верно и в отношении ее централизатора $Z_{G}(H)$. Поэтому подпространство $\pi\left(Z_{G}(H)\right) \subset G_{\mathbb{Z}} \backslash G$ замкнуто. Если оно некомпактно, то найдется последовательность $z_{i} \in Z_{G}(H)$ такая, что $\pi\left(z_{i}\right) \rightarrow \infty$, т.е. для любых двух компактов $K \subset G_{\mathbb{Z}} \backslash G, C \subset \pi(H)$ пересечение $C z_{i} \cap K$ пусто для всех $i$ за исключением конечного числа. Поэтому $\mu_{H} z_{i}(K) \rightarrow 0, i \rightarrow \infty$, для любого компакта $K \subset G_{\mathbb{Z}} \backslash G$, т.е. $\mu_{H} z_{i} \rightarrow 0$. Мы показали, что семейство $\left\{\mu_{H} g, g \in G\right\}$ не является предкомпактньм, если $\pi\left(Z_{G}(H)\right)$ некомпактно. С другой стороны, имеет место

TeOPEмa 9.7 ([EMS97]). Пусть $H \subset G \subset S L(n, \mathbb{R})$ - две редуктивныее алгебраические $\mathbb{Q}$-группьи, причем $H_{\mathbb{Z}}$ и $G_{\mathbb{Z}}$ - решетки в $H$ и $G$ соответственно. Тогда если $\pi\left(Z_{G}(H)\right)$ компактно, то для каждого $\varepsilon>0$ найдется компакт $K \subset G_{\mathbb{Z}} \backslash G$ такой, что $\mu_{H} g(K)>1-\varepsilon$ для любого $g \in G$. 
Таким образом, мы получили критерий предкомпактности семейства вероятностных мер $\left\{\mu_{H} g, g \in G\right\}$. В доказательстве приходится обобщать теорему 9.5 Дани и Маргулиса о возвращаемости унипотентных траекторий в некоторьй компакт. Заметим, что условие компактности $\pi\left(Z_{G}(H)\right)$ эквивалентно любому из следуюших двух: 1) $H$ не содержится в параболической $\mathbb{Q}$-подгруппе $G$; 2) любая $\mathbb{Q}$-подгруппа в $G$, содержашая $H$, редуктивна.

Ответ на второй вопрос (но не само доказательство) оказьвается на редкость простым:

TEOPEMA 9.8 ([EMS96]). Пусть $H \subset G \subset S L(n, \mathbb{R})$ - две алгебраические $\mathbb{Q}$-группьи, причем $H_{\mathbb{Z}}$ и $G_{\mathbb{Z}}$ - решетки в $H$ и $G$ соответственно. Тогда любая предельная вероятностная мера для семейства $\left\{\mu_{H} g, g \in G\right\}$ является алгебраической. Более того, если $\mu_{H} g_{i} \rightarrow \mu \in \mathscr{P}\left(G_{\mathbb{Z}} \backslash G\right)$, то найдутся алгебраическая $\mathbb{Q}$-группа $L \subset G$ и последовательности $\gamma_{i} \in G_{\mathbb{Z}} u c_{i} \rightarrow c \in G$ такие, что $\mu$ является $c^{-1} L c$-инвариантной мерой на $\pi(L) c, \gamma_{i}^{-1} H \gamma_{i} \subset L$ и $H g_{i}=H \gamma_{i} c_{i}$ для всех $i$ кроме конечного их числа.

Доказательство теоремы основано на следующем наблюдении [EMS96]: либо найдется последовательность $c_{i} \rightarrow c$ такая, что $\mu_{H} g_{i}=\mu_{H} c_{i}$ (и тогда $\mu=\mu_{H} c$ ), либо $\mu$ инвариантна относительно некоторой унипотентной подгрупшы в $G$ (и тогда можно использовать хорошо развитую технику унипотентных потоков). Заметим, что в данном результате редуктивность $H$ и $G$ не нужна.

Фактически вторая часть теоремы дает ответ и на первый вопрос. Иногда, впрочем, никакие проверки на существование промежуточных подгрупा $L \subset G$ и не требуются:

СлЕДСТВИЕ 9.9 ([ЕMS96]). В условиях теоремы 9.8 пусть ная связная алгебраическая $\mathbb{Q}$-группа. Тогда $\mu_{H} g_{i} \rightarrow \mu_{G}$, как только $\mathrm{Hg}_{i} \rightarrow \infty$ в $H \backslash G$.

6. Теперь мы обсудим работу Шаха [Sh94], в которой доказьвается довольно неожиданньй результат о том, что алгебраичность орбит и их равномерная распределенность сохраняют свою силу, если вместо действий унипотентных потоков мы рассмотрим полиномиальные кривые. Идея состоит в том, что при доказательстве теоремы 8.1, по сушеству, использовалась лишь полиномиальная расходимость унипотентных траекторий. Скажем, что $\theta: \mathbb{R} \rightarrow S L(n, \mathbb{R})$ - полином, если каждая координатная функция $\theta_{i, j}$ является полиномиальной.

Теорема 9.10 ([Sh94]). Пусть $G \subset S L(n, \mathbb{R})$ - замкнутая подгруппа с решеткой $\Gamma \subset G$ и $\theta: \mathbb{R} \rightarrow G \subset S L(n, \mathbb{R})$ - такой полином, ито $\theta(0)=1 \in G$. Тогда существует замкнутая подгруппа $F \subset G$ такая, что орбита $Г F$ имеет вероятностную $F$-инвариантную меру $\nu_{F} u \mu_{T, \Gamma, \theta} \rightarrow \nu_{F}$ nри $T \rightarrow \infty$.

Идея доказательства состоит в следующем. Пусть $\mu$ есть предельная мера для некоторой последовательности $\left\{\mu_{T_{n}}\right\}$. Оказьвается, эта мера инвариантна относительно некоторой унипотентной подгруппы $u_{\mathbb{R}} \subset G$. Это вытекает из следующих двух наблюдений: 
Лемма 9.11 ([Sh94]). Пусть $\theta: \mathbb{R} \rightarrow S L(n, \mathbb{R})$ - нетривиальный полином. Тогда найдутся $q \geqslant 0$ и нетривиальная унипотентная подгруппа $u: \mathbb{R} \rightarrow S L(n, \mathbb{R})$ такие, что $\lim _{t \rightarrow \infty} \theta(t)^{-1} \theta\left(t+s t^{-q}\right)=u(s)$ для любого $s \in \mathbb{R}$.

Для иллюстрации: если $\theta(t)=\left(\begin{array}{cc}1 & a_{0} t^{n}+a_{1} t^{n-1}+\cdots \\ 0 & 1\end{array}\right), a_{0} \neq 0$, то $q=n-1$, $u(s)=\left(\begin{array}{cc}1 & a_{0} n s \\ 0 & 1\end{array}\right)$.

ЛЕмма 9.12 ([Sh94]). $\lim _{T \rightarrow \infty} \frac{1}{T} \int_{1}^{T}\left(f\left(t+s t^{-q}\right)-f(t)\right) d t=0$ для любых $f \in C_{c}(\mathbb{R})$, $q \geqslant 0, s \in \mathbb{R}$, причем скорость сходимости зависит только от $s, q u \sup |f|$.

Докажем, что предельная мера $\mu$ инвариантна относительно $u_{\mathbb{R}}$. В самом деле,

$$
\begin{aligned}
\int_{\Gamma \backslash G} f(x u(s)) d \mu_{x} & =\lim _{n \rightarrow \infty} \frac{1}{T_{n}} \int_{0}^{T_{n}} f(\Gamma \theta(t) u(s)) d t \\
& =\lim _{n \rightarrow \infty} \frac{1}{T_{n}} \int_{0}^{T_{n}} f\left(\Gamma \theta\left(t+s t^{-q}\right)\right) d t \\
& =\lim _{n \rightarrow \infty} \frac{1}{T_{n}} \int_{0}^{T_{n}} f(\Gamma \theta(t)) d t=\int_{\Gamma \backslash G} f(x) d \mu_{x},
\end{aligned}
$$

где второе равенство следует из выбора $u_{\mathbb{R}}$ и равномерной непрерьвности $f$, а третье - из леммы 9.12.

Таким образом, предельная мера $\mu$ инвариантна относительно унипотентной подгрупы, и потому каждая ее эргодическая компонента имеет алгебраическое происхождение. Дальнейшие рассуждения аналогичны доказательству равномерной распределенности унипотентных траекторий в $\S 8$. А именно, теорема D.8 о том, что унипотентная траектория на пространстве конечного объема почти все свое время проводит внутри некоторого компакта, использует лишь полиномиальность траектории, откуда выводится свойство $\mu(\Gamma \backslash G)=1$. Затем используется теорема 8.5 о счетности, процедура линеаризации и лемма 8.6 о поведении полиномиальных траекторий вблизи аффинных многообразий в векторных пространствах.

Теорема о равномерной распределенности имеет место и для полиномов от нескольких переменных. Назовем параллелепипедом $B \subset \mathbb{R}^{k}$ множество вида $\left[0, T_{1}\right] \times \cdots \times$ $\left[0, T_{k}\right]$ и рассмотрим отображение $\Theta: \mathbb{R}^{k} \rightarrow G \subset S L(n, \mathbb{R})$, которое имеет вид произведения $\Theta\left(t_{1}, \ldots, t_{k}\right)=\theta_{1}\left(t_{1}\right) \cdots \theta_{k}\left(t_{k}\right)$, где $\theta_{1}, \ldots, \theta_{k}$ - набор из $k$ полиномов. Возьмем последовательность мер

$$
\mu_{B_{n}}(f)=\frac{1}{l\left(B_{n}\right)} \int_{t \in B_{n}} f(\Gamma \Theta(t)) d l(t), \quad f \in C_{c}(\Gamma \backslash G),
$$

построенную по последовательности параллелепипедов в $\mathbb{R}^{k}$. Как показано в [Sh94], имеет место сходимость $\mu_{B_{n}} \rightarrow \nu_{F}$ к $F$-инвариантной мере $\nu_{F}$ на $\Gamma F$ для некоторой подгрупшы $F \subset G$; здесь $t=\left(t_{1}, \ldots, t_{k}\right) \in \mathbb{R}^{k}, l$ - лебеговская мера на $\mathbb{R}^{k}$ и $\left\{B_{n}\right\}$ - последовательность $k$-мерных параллелепипедов в $\mathbb{R}^{k}$, длины всех сторон которых стремятся к бесконечности (независимо друг от друга). В качестве $\Theta$ можно взять 
и произвольное полиномиальное отображение $\mathbb{R}^{k}$ в $S L(n, \mathbb{R})$, но тогда в качестве $B_{n}$ нужно брать последовательность шаров в $\mathbb{R}^{k}$ с возрастающими радиусами.

Из этих результатов вьводится и утверждение о равномерной распределенности орбит многомерных унипотентных подгрупп, которое было доказано Ратнер в разрешимом случае [R91b] и высказано ею в качестве гипотезы в обшем случае в [R94]. Скажем, что базис $B=\left\{b_{1}, \ldots, b_{k}\right\}$ в нильпотентной алгебре Ли $\mathfrak{n}$ является треугольным, если структурные константы удовлетворяют равенству $c_{i, j}^{l}=0$, как только $l \leqslant \max (i, j)$ (если подалгебра $\mathfrak{n}_{l}$ натянута на $b_{l}, \ldots, b_{k}$, то она является идеалом и $\left[\mathfrak{n}, \mathfrak{n}_{l}\right] \subset \mathfrak{n}_{l+1} ;$ ясно, что такой базис сушествует). Перестановка треугольного базиса называется регулярным базисом в $\mathfrak{n}$.

ТеОрема 9.13 ([Sh94]). Пусть $\Gamma$ - решетка в группе Ли $G$ и $N \subset G$ - односвязная унипотентная подгруппа в $G$. Рассмотрим регулярный базис $\left\{b_{1}, \ldots, b_{k}\right\}$ в алгебре Ли $\mathfrak{n}$ и отображение $S: \mathbb{R}^{k} \rightarrow N$, заданное по правилу $S\left(t_{1}, \ldots, t_{k}\right)=$ $\exp \left(t_{1} b_{1}\right) \cdots \exp \left(t_{k} b_{k}\right)$. Для любого параллелепипеда $B \subset \mathbb{R}^{k}$ положим

$$
\mu_{B}(f)=\frac{1}{\lambda(S(B))} \int_{t \in B} f\left(\Gamma S\left(t_{1}, \ldots, t_{k}\right)\right) d t,
$$

где $\lambda$ обозначает меру Хаара на $N$. Тогда найдется подгруппа $F \subset G$ такая, что для любой последовательности параллелепипедов $\left\{B_{n}\right\} \subset \mathbb{R}^{k}$, длины всех сторон которых стремятся к бесконечности, имеет место сходимость $\mu_{B_{n}} \rightarrow \nu_{F}$ ж F-инвариантной мере на орбите ГF.

Если $G$ - вещественно-алгебраическая група Ли, то данньй результат очевидно следует из предыдущих, так как для регулярного базиса отображение $S$ переводит лебегову меру на $\mathbb{R}^{k}$ в меру Хаара на $N$ (см. [R90b]). Случай произвольной группы Ли $G$ сводится к алгебраическому.

Отметим один нерешенньй вопрос из данной области. Что будет, если вместо полиномиальной траектории $\Gamma \theta(\mathbb{R}) \subset \Gamma \backslash G$ рассмотреть $\Gamma \theta(\mathbb{Z}) ?$ Если $\theta: \mathbb{R} \rightarrow G$ является унипотентной подгруппой, то речь идет о равномерной распределенности орбит унипотентных каскадов, которая имеет место в силу 8.1. С другой стороны, если $\theta: \mathbb{R} \rightarrow \mathbb{R}^{n}$ - произвольное полиномиальное отображение, то по теореме Вейля $[\mathrm{KSF}]$ равномерная распределенность на торе $\mathbb{Z}^{n} \backslash \mathbb{R}^{n}$ имеет место как для $x \theta(\mathbb{R})$, так и для $x \theta(\mathbb{Z}), x \in \mathbb{Z}^{n} \backslash \mathbb{R}^{n}$. Однако если $G=S L(2, \mathbb{R}), \Gamma=S L(2, \mathbb{Z})$ и, скажем, $\theta(t)=$ $\left(\begin{array}{ll}1 & t^{2} \\ 0 & 1\end{array}\right)$, то устройство счетных “траекторий” $\Gamma g \theta(\mathbb{Z}) \subset \Gamma \backslash G$ пока не изучено. Будут ли их замькания алгебраичны и будут ли они равномерно распределены в своих замьканиях?

\section{$\S$ 10. Структура орбит, минимальных множеств и эргодических мер однородных потоков}

В этом параграфе содержится обзор работ автора [St90], [St95a] о строении орбит, минимальных множеств и конечных эргодических мер однопараметрических однородных потоков. Еще раз напомним, что все замыкания орбит и все конечные эргодические меры унипотентного потока на пространстве конечного объема являются однородными. Несколько более общий класс потоков составляют квазиунипотентные потоки, 
и мы покажем, что замыкание произвольной орбиты такого потока, хотя и не обязано быть однородным, является гладким многообразием (и более того, имеет вид инфраоднородного пространства $\Gamma \backslash F / C$, где $C$ - компактная подгруппа в групше $\mathrm{Aff}(\Gamma \backslash F)$ всех аффинных отображений $\Gamma \backslash F)$. Далее, это многообразие (вместе с потоком) компактно накрьвается однородньм пространством конечного объема. Отсюда легко вывести свойство равномерной распределенности квазиунипотентных орбит и тот факт, что произвольная конечная эргодическая мера является гладкой мерой, сосредоточенной на некотором подмногообразии. Кроме того, мы обобшим топологическую теорему Ратнер на класс тех орбит произвольных (возможно, частично-гиперболических) потоков, которые “не уходят на бесконечность внутри нейтрального слоя". Такое усиление оказывается необходимьм для классификации минимальных множеств.

Далее $\Gamma$ - дискретная подгруппа в группе Ли $G$. Напомним, что динамика однородного потока $\left(\Gamma \backslash G, g_{\mathbb{R}}\right)$ в решаюшей степени определяется тем, является ли подгруппа $g_{\mathbb{R}}$ квазиунипотентной или нет. Относительно метрики, индуцированной левоинвариантной метрикой на $G$, скорость разбегания траекторий квазиунипотентного потока $\left(\Gamma \backslash G, g_{\mathbb{R}}\right)$ является квазиполиномиальной (с возможньм присутствием тригонометрической части), в то время как неквазиунипотентньй поток имеет экспоненциальную расходимость траекторий и является равномерно частично-гиперболическим.

1. Как уже отмечалось, орбиты геодезического потока могут быть “весьма плохими”. А именно, орбита может быть нерекуррентной и не уходяшей на бесконечность или, напротив, быть рекуррентной и иметь локально-несвязное замыкание (как морсовское минимальное множество). Обобщением этого факта является следующее наблюдение Маргулиса.

ЛЕМма 10.1 ([St90]). Любой консервативный равномерно частично-гиперболический поток на многообразии всегда имеет нерекуррентную орбиту, не уходящую на бесконечность (и поэтому ее замыкание не является подмногообразием).

ЗАмЕчАНИЕ. Было бы интересно у частично-гиперболических потоков (хотя бы однородных) найти также и орбиты с локально-несвязным замыканием. Такие орбиты заведомо имеются у аносовских потоков (например, в окрестности замыкания гомоклинической орбиты; подробнее см. [AS]).

Что касается квазиунипотентных потоков, их орбиты можно наглядно представлять как “искривления" орбит унипотентных потоков. В самом деле, если, например, группа $G$ полупроста и имеет конечный центр, то для любой квазиунипотентной подгрупшы $g_{\mathbb{R}} \subset G$ найдется тор $T \subset G$ и унипотентная подгруппа $u_{\mathbb{R}} \subset G$ такие, что $g_{t}=c_{t} \times u_{t}, t \in \mathbb{R}$, где $c_{\mathbb{R}}-$ плотная подгруппа в торе $T$ (мы получили так назьваемое разложение Жордана подгруппы $g_{\mathbb{R}}$ в редуктивную и унипотентную части). Таким образом, подгруппу $g_{\mathbb{R}}$ можно представлять как диагональную в “цилиндре" $T \times u_{\mathbb{R}}$ с образующей $u_{\mathbb{R}}$.

В общем случае внутри $G$ такого разложения может и не быть. Однако можно “слегка" расширить группу $G$ до группы $G^{*}$, в которой уже есть нужное нам разложение.

ПримеР 10.2. Пусть $G=\mathbb{R} \cdot \mathbb{R}^{2}$ - разрешимая группа Ли, в которой $\mathbb{R}$ действует на плоскости $\mathbb{R}^{2}$ ортогональными врашениями с ядром $2 \pi \mathbb{Z} \subset \mathbb{R}$. Рассмотрим 
решетку $\Gamma=Z(a) \times \mathbb{Z}^{2}$, где $Z(a)$ - циклическая группа, порожденная элементом $a=2 \pi \times(\sqrt{2}, 0)$. Пусть $g_{t}=t \in \mathbb{R}$. Тогда $g_{\mathbb{R}}$ является квазиунипотентной (Ad-редуктивной) подгруппой, но внутри $G$ мы не можем представить ее диагональной подгруппй в цилиндре вида $T \times u_{\mathbb{R}}$ с тором $T$ и унипотентной подгруппой $u_{\mathbb{R}}$. Положим $G^{*}=S O(2) \cdot G$, где $S O(2)$ коммутирует с $\mathbb{R}$ и действует ортогональными вращениями на $\mathbb{R}^{2}$. Действие элемента $t \in \mathbb{R}$ на $\mathbb{R}^{2}$ совпадает с действием элемента $t^{*} \in S O(2)$ (и есть поворот на угол $t$ ). Пусть $\Delta=\left\{\left(t,-t^{*}\right), t \in \mathbb{R}\right\}$ - "антидиагональ" в $\mathbb{R} \times S O(2)$. Тогда $\Delta$ является центром в $G^{*}$ и $g_{t}=c_{t} \times u_{t}$, где $c_{t}=t^{*} \in S O(2)$ и $u_{t}=\left(t,-t^{*}\right) \in \Delta$. Так как $\Gamma \subset N=\Delta \times \mathbb{R}^{2}$, то проекция П: $S O(2) \cdot N \rightarrow N$ индуцирует диффеоморфизм $\Pi^{*}: \Gamma \backslash G \rightarrow \Gamma \backslash N$. Так как $S O(2)$ коммутирует с $g_{\mathbb{R}}$, то П переводит $g_{t}$-действие в $u_{t}$-действие и тем самым индуцирует гладкий изоморфизм потоков $\left(\Gamma \backslash G, g_{\mathbb{R}}\right)$ и $\left(\Gamma \backslash N, u_{\mathbb{R}}\right)$. Так как $\left(\overline{\Gamma u_{\mathbb{R}}}\right)_{0}=\Delta \times \mathbb{R}^{\prime}$, где прямая $\mathbb{R}^{\prime}$ натянута на $(1,0)$, то замькание $\overline{\Gamma g_{\mathbb{R}}} \subset \Gamma \backslash G$ совпадает с многообразием $\Gamma\left(\mathbb{R} \mathbb{R}^{\prime}\right) \subset \Gamma \backslash G$, которое диффеоморфно двумерному тору $\Gamma\left(\Delta \times \mathbb{R}^{\prime}\right) \subset \Gamma \backslash N$, но само не является однородным подпространством в $\Gamma \backslash G$. Заметим, что, взяв другую абелеву решетку $\Gamma=Z(a) \times \mathbb{Z}^{2}$, где $a=2 \pi \times(\sqrt{2}, \sqrt{3})$, мы получили бы гладкий изоморфизм минимального потока на разрешимом пространстве $\Gamma \backslash G$ с минимальным прямолинейньм потоком на трехмерном торе $\Gamma \backslash N$.

В общем случае имеет место

ЛЕмма 10.3 ([St90]). Пусть $g_{\mathbb{R}}-$ квазиунипотентная подгруппа в группе Ли $G$. Тогда найдутся

(1) группа Ли $G^{*}$, которая содержит $G$ в качестве нормальной подгруппь, причем $G^{*} / G$ является тором, и

(2) унипотентная подгруппа $h_{\mathbb{R}} \subset G^{*}$

такие, что для произвольной дискретной подгруппь $\Gamma \subset G$ каждое замькание $M=\overline{x g_{\mathbb{R}}} \subset \Gamma \backslash G$ компактно накрывается замыканием $M^{\prime}=\overline{x h_{\mathbb{R}}} \subset \Gamma \backslash G^{*}$, причем накрытие является сопряженностью потоков. Если $M^{\prime}$ - однородное пространство конечного оббема, то $M-$ гладкое многообразие.

Ясно, что $\operatorname{vol}(\Gamma \backslash G)<\infty \Longleftrightarrow \operatorname{vol}\left(\Gamma \backslash G^{*}\right)<\infty$, и поэтому для решетки $\Gamma \subset G$ все орбиты унипотентного потока $\left(\Gamma \backslash G^{*}, h_{\mathbb{R}}\right)$ имеют однородные замыкания и свойство равномерной распределенности. Отсюда сразу вытекает, что произвольная орбита $x g_{\mathbb{R}}$ квазиунипотентного потока на пространстве конечного объема также равномерно распределена (относительно гладкой меры на многообразии $M=\overline{x g_{\mathbb{R}}} \subset \Gamma \backslash G$, полученной из $H$-инвариантной меры на пространстве $\left.M^{\prime}=x H \subset \Gamma \backslash G^{*}\right)$. Заметим также, что многообразие $M$ не обязано быть однородным подпространством (см. пример 10.2).

Теперь мы можем сформулировать обший критерий.

TeOpema 10.4 ([St90]). Пусть Г - решетка в G. Тогда все орбить потока $\left(\Gamma \backslash G, g_{\mathbb{R}}\right)$ имеют гладкие замыкания $\Longleftrightarrow$ подгруппа $g_{\mathbb{R}}$ квазиунипотентна.

2. Пусть теперь $\mu$ - конечная эргодическая мера для квазиунипотентного потока $\left(\Gamma \backslash G, g_{\mathbb{R}}\right)$ и $M \subset \Gamma \backslash G$ - ее носитель (здесь мы не предполагаем, что $\left.\operatorname{vol}(\Gamma \backslash G)<\infty\right)$. Тогда поток $\left(M, g_{\mathbb{R}}\right)$ является эргодическим по отношению к строго положительной мере, и поэтому он имеет плотную орбиту. Но тогда по лемме 10.3 поток $\left(M, g_{\mathbb{R}}\right)$ 
компактно накрьвается топологически транзитивньм потоком вида $\left(M^{\prime}, h_{\mathbb{R}}\right)$, где $M^{\prime} \subset \Gamma \backslash G^{*}$ и $h_{\mathbb{R}}-$ унипотентная подгруппа в $G^{*}$. Конструкция Вейля полупрямого произведения мер (cм. [Rag]) снабжает нас конечной $h_{\mathbb{R}}$-инвариантной мерой на $M^{\prime}$, которая проектируется в меру $\mu$. Пусть $\mu^{\prime \prime}$ - та эргодическая компонента $\mu^{\prime}$, которая проектируется на $\mu$. Тогда $\mu^{\prime \prime}$ является $H$-инвариантной мерой на некоторой замкнутой орбите $x H \subset M^{\prime} \subset \Gamma \backslash G^{*}$. Поэтому $\mu$ является гладкой мерой на многообразии $M \subset \Gamma \backslash G$. Мы вьвели

СлЕДСТВИЕ 10.5. Пусть $\mu$ - конечная әргодическая мера для квазиунипотентного потока $\left(\Gamma \backslash G, g_{\mathbb{R}}\right)$. Тогда найдется гладкое многообразие $M \subset \Gamma \backslash G$ такое, что н сосредоточена на $M$ и ограничение $\mu$ на $M$ является гладкой мерой, равномерно распределенной относительно потока.

Напомним еще раз, что согласно Синаю [Si72] и Боуэну [Во75] каждый аносовский поток имеет континуум эргодических мер как с локально-несвязным носителем, так и строго положительных (но сингулярных к гладкой мере объема). Простейший пример строго положительных эргодических мер построен в работе Аносова [An]. В этом примере берется гиперболический автоморфизм тора $A: T^{2} \rightarrow T^{2}$ и строится непрерьвное семейство гладких возмушений $A_{s}: T^{2} \rightarrow T^{2}, A_{0}=A$, причем лебеговская мера $l$ на $T^{2}$ остается инвариантной. При этом энтропия $A_{s}$ меняется непрерывно и в точке $s=0$ достигает своего локального максимума. По теореме Аносова о грубости для достаточно малых $s$ найдется гомеоморфизм $\varphi_{s}: T^{2} \rightarrow T^{2}$ такой, что $\varphi_{s} A_{s}=A \varphi_{s}$. Легко видеть, что каждая мера $\left(\varphi_{s}\right)_{*}(l)$ строго положительна, эргодична и отлична от $l$ из соображений энтропии. Ясно, что надстройка над $A$ есть однородный аносовский поток на 3-мерном разрешимом многообразии и для него также имеется континуум эргодических мер.

Уместно высказать следуюшее предположение.

ГИПОТЕЗА. Любой частично-гиперболический поток на однородном пространстве конечного обгема имеет конечную эргодическую меру, носитель которой не является гладким многообразием. Если он эргодичен, то найдется эргодическая строго положительная негладкая мера.

3. Перейдем теперь к орбитам частично-гиперболических однородных потоков. В случае $\operatorname{vol}(\Gamma \backslash G)<\infty$ почти все орбиты имеют гладкое замыкание (для эргодических потоков типичные орбиты всюду плотны, а для неэргодических совпадают с соответствуюшим эргодическим подмногообразием [St83]). Среди оставшихся орбит есть орбиты как с негладким (см. лемму 10.1), так и с гладким замьканием (например, периодические орбиты геодезического потока). Оказьвается, при некоторых условиях можно гарантировать гладкость или даже однородность замыканий некоторых орбит потока $\left(\Gamma \backslash G, g_{\mathbb{R}}\right)$.

Чтобы сфформулировать результат, рассмотрим разложение $\mathfrak{g}=\mathfrak{g}^{-}+\mathfrak{g}^{0}+\mathfrak{g}^{+}$алгебры Ли $\mathfrak{g}$ групшы $G$ на сжимаюшуюся, нейтральную и расширяюшуюся подалгебры по отношению к оператору $\operatorname{Ad}_{g_{1}}$. Обозначим через $G^{-}, Q$ и $G^{+}$соответствуюшие аналитические подгруппы в $G$. Мы знаем, что орбиты $G^{-}, Q$ и $G^{+}$на $\Gamma \backslash G$ образуют соответственно сжимаюшееся, нейтральное и расширяюшееся слоения для потока $\left(\Gamma \backslash G, g_{\mathbb{R}}\right)$. Заметим, что $g_{\mathbb{R}} \subset Q$ и $g_{\mathbb{R}}$ всегда квазиунипотентна в своей нейтральной 
подгруппе $Q$. Скажем, что орбита $x g_{\mathbb{R}} \subset \Gamma \backslash G$ не уходит на бесконечность внутри нейтрального слоя $x Q \subset \Gamma \backslash G$, если найдутся последовательность $\left\{t_{n}\right\} \rightarrow \infty$ и компакт $K \subset Q$ такие, что $x g_{t_{n}} \in x K$ для всех $n$. Можно доказать следующее.

TEOPEмa 10.6 ([St95a]). Предположим, что $\operatorname{vol}(\Gamma \backslash G)<\infty$ и орбита $x g_{\mathbb{R}} \subset$ $\Gamma \backslash G$ не уходит на бесконечность внутри нейтрального слоя $x Q \subset \Gamma \backslash G$. Тогда замыкание $\overline{x g_{\mathbb{R}}} \subset \Gamma \backslash G$ является гладким многообразием.

Доказательство этой теоремы использует утверждение, аналогичное лемме 10.3. А именно, найдутся группа Ли $G^{*}$ и подгруппа $h_{\mathbb{R}} \subset G^{*}$ такие, что $G^{*} / G$ - тор, все собственные значения операторов $\mathrm{Ad}_{h_{t}}$ на $\mathfrak{g}^{*}$ являются чисто вешественньми и каждое замькание $\overline{x g_{\mathbb{R}}} \subset \Gamma \backslash G$ компактно накрывается замыканием $\overline{x h_{\mathbb{R}}} \subset \Gamma \backslash G^{*}$. Если второе замыкание однородно, то первое является гладким. Теперь теорема 10.6 вытекает из следующего результата.

Tеорема 10.7 ([St95a]). Предположим, что $\operatorname{vol}(\Gamma \backslash G)<\infty$ и подгруппа $g_{\mathbb{R}}$ maкова, что все собственные значения операторов $\operatorname{Ad}_{g_{t}}$ на $\mathfrak{g}$ чисто вещественные. Тогда если орбита $x g_{\mathbb{R}} \subset \Gamma \backslash G$ не уходит на бесконечность внутри нейтрального слоя $x Q \subset \Gamma \backslash G$, то замыкание $\overline{x g_{\mathbb{R}}} \subset \Gamma \backslash G$ является однородным пространством конечного обгема.

Заметим, что данная теорема обобщает топологическую теорему Ратнер для однопараметрических унипотентных потоков. В самом деле, если $\operatorname{vol}(\Gamma \backslash G)<\infty$, то по лемме Маргулиса D.7 ни одна из полуорбит унипотентного потока не уходит на бесконечность. Теорема 10.7 не является непосредственным следствием теоремы Ратнер, так как нейтральный слой $x Q \subset \Gamma \backslash G$ может быть незамкнутым и, как однородное $Q$-пространство, иметь бесконечную $Q$-инвариантную меру. Теорема 10.7 доказывается многократньм применением теоремы Ратнер (вначале с использованием теории алгебраических груп рассматривается случай арифметической подгруппы $\Gamma=G \cap S L(n, \mathbb{Z})$, а затем полупростой, разрешимый и обший случаи сводятся к арифметическому). Казалось бы, ее можно вьвести из теоремы 8.3 Дани и Маргулиса следующим образом. Пусть $x=\Gamma \in \Gamma \backslash G$. Рассмотрим унипотентный поток $\left((\Gamma \cap Q) \backslash Q, g_{\mathbb{R}}\right)$ на пространстве $(\Gamma \cap Q) \backslash Q$ (вообще говоря, бесконечного объема). По условию орбита $(\Gamma \cap Q) g_{\mathbb{R}}$ не уходит на бесконечность и поэтому через сколь угодно большое время возврашается в некоторьй фиксированньй компакт. Если бы эта орбита возвращалась в данньй компакт с положительной плотностью моментов возврашения, мы могли бы применить теорему 8.3 и немедленно вывести наш результат. Однако орбита, скажем, орициклического потока может не уходить на бесконечность и в то же время не возврашаться в какой-либо компакт с положительной плотностью времен (так устроены, например, рекуррентные непериодические орбиты орициклического потока на поверхности второго рода, см. [St95b]).

$\mathrm{C}$ другой стороны, заметим, что для редуктивной (т.е. состоящей из Ad-полупростых элементов) подгрупшы $g_{\mathbb{R}} \subset G$ теорема 10.7 почти тривиальна, так как $g_{\mathbb{R}}$ лежит в центре своей нейтральной подгрупшы $Q$ и потому орбита $(\Gamma \cap Q) g_{\mathbb{R}}$ не уходит на бесконечность в $(\Gamma \cap Q) \backslash Q$, лишь если ее замыкание является тором. Таким образом, основное значение данная теорема имеет для “смешанных" (не редуктивных и не унипотентных) подгрупп $g_{\mathbb{R}} \subset G$. 
4. Теперь мы в состоянии дать классификацию минимальных множеств однородных потоков. Вначале введем новьй класс инвариантных множеств.

Пусть $\left(X, g_{\mathbb{R}}\right)$ - непрерьвньй поток на локально-компактномпространстве $X$. Тогда замкнутое инвариантное множество $M \subset X$ называется бирекуррентным, если каждая орбита внутри $M$ рекуррентна (возврашается сколь угодно близко к исходной точке) в обоих направлениях.

Этот класс инвариантных множеств естественно возникает в топологической динамике. Ясно, что любое компактное минимальное множество является бирекуррентным. Пример Бебутова (см. [Ne] или $[\mathrm{KW}]$ ) показывает, что компактное бирекуррентное множество не обязано быть минимальным, даже если оно топологически транзитивно. Однако в этом примере множество имело бесконечную размерность. Теперь, имея в распоряжении теорему Ратнер, мы можем строить алгебраические примеры таких множеств. В самом деле, компактное однородное пространство конечного объема является бирекуррентным по отношению к любому унипотентному потоку на нем, но эргодический унипотентный поток не обязан быть минимальным. (Пример: $\Gamma \subset S L(4, \mathbb{R})$ - равномерная решетка, которая пересекает $S L(2, \mathbb{R})$ по решетке, и $g_{\mathbb{R}} \subset S L(2, \mathbb{R})$ - любая унипотентная подгруппа. Тогда поток $\left(\Gamma \backslash S L(4, \mathbb{R}), g_{\mathbb{R}}\right)$ эргодичен и неминимален.)

Доказательство следующего результата смоделировано над леммой Маргулиca 10.1 .

Лемма 10.8 ([St95a]). Пусть $\left(X, g_{\mathbb{R}}\right)$ - равномерно частично-гиперболический поток на многообразии $X$ с бирекуррентныцм множеством $M \subset X$. Пусть $O(x) \subset X-$ достаточно малая окрестность точки $x \in M$. Тогда связная компонента точки $x$ в пересечении $M \cap O(x)$ лежит внутри нейтрального слоя $x \in X$.

Из теоремы 10.6 и леммы 10.8 немедленно вытекает

Теорема 10.9 ([St95a]). Пусть $M$ - топологически транзитивное бирекуррентное множество для потока $\left(\Gamma \backslash G, g_{\mathbb{R}}\right)$ на однородном пространстве конечного обгема. Тогда либо $M$ локально-несвязно в каждой точке $x \in M$, либо $M$ является гладким подмногообразием в $\Gamma \backslash G$.

Теперь предположим, что $M$ - минимальное множество непрерывного потока $\left(X, g_{\mathbb{R}}\right)$. Тогда либо все полуорбиты внутри $M$ не уходят на бесконечность, либо хотя бы одна из них уходит. Маргулис в [Ма91а] доказал, что в первом случае $M$ обязано быть компактом. Теперь мы можем дать классификацию минимальных множеств однородных потоков.

Tеорема 10.10 ([St95a]). Пусть $M$ - минимальное множество однородного потока $\left(\Gamma \backslash G, g_{\mathbb{R}}\right)$. Тогда выполнено одно из следующих условий:

1) $M=x g_{\mathbb{R}}$ есть орбита, уходящая на бесконечность в обоих направлениях,

2) найдется точка $x \in M$ такая, что одна из ее полуорбит уходит на бесконечность, а другая является рекуррентной,

3) $M$ - компактное локально-несвязное в каждой точке множество, 
4) $M$ - компактное гладкое подмногообразие в $\Gamma \backslash G$ такое, что поток $\left(M, g_{\mathbb{R}}\right)$ компактно накрывается строго эргодическим унипотентным потоком.

Заметим, что данная теорема имеет место для произвольных дискретных подгрупп $\Gamma \subset G$. Если $\Gamma \backslash G$ не имеет конечного объема, то вместо теоремы Ратнер мы можем применить теорему 8.3 Дани и Маргулиса (согласно которой компактные замькания унипотентных орбит всегда однородны)

Предположим теперь, что $\operatorname{vol}(\Gamma \backslash G)<\infty$. Из теорем Ратнер следует, что для унипотентного однопараметрического потока свойства минимальности и строгой эргодичности эквивалентны. Из предыдущей теоремы вытекает, что любой квазиунипотентньй поток может быть минимальным, лишш если $\Gamma \backslash G$ - компакт, и в этом случае поток строго эргодичен. С другой стороны, из леммы 10.1 следует, что однородный поток может быть минимальньм, лишь если он квазиунипотентен. Таким образом, из минимальности следует строгая эргодичность. Обратно, как недавно доказали Клейнбок и Маргулис [КМ96], однородньй поток на пространстве конечного объема всегда обладает ограниченными (т.е. предкомпактными) орбитами и поэтому обладает компактным инвариантньм множеством. Хорошо известно, что на таком множестве всегда есть хотя бы одна инвариантная мера, и поэтому строго эргодичным может быть лишь поток на компактном пространстве. Но для однопараметрических потоков на компакте строгая эргодичность относительно $G$-инвариантной меры влечет минимальность. Мы доказали следующее.

ТеоРема 10.11. Для однопараметрических потоков на пространстве конечного обгема минимальность и строгая эргодичность эквивалентны.

Орбиты геодезического потока, уходящие на бесконечность в обоих направлениях, представляют собой первый тип минимальных множеств по нашей классификации, морсовские минимальные множества - третий, а периодические орбиты - четвертый. Кажется вероятным, что (если поверхность имеет конечную площадь и некомпактна) геодезический поток обладает и минимальными множествами второго типа.

Пусть теперь $\left(\Gamma \backslash G, g_{\mathbb{R}}\right)$ - произвольньй эргодический поток на однородном пространстве конечного объема. Имеет ли он хотя бы одно минимальное множество? Конечно, если пространство $\Gamma \backslash G$ компактно, у нас нет проблем. Если же оно некомпактно и подгруппа $g_{\mathbb{R}}$ не является квазиунипотентной либо редуктивной, вопрос оказался не столь тривиален.

В общем случае существование ограниченных орбит (а следовательно, и компактных минимальных множеств) было доказано Клейнбоком и Маргулисом [KM96]. Остановимся чуть подробнее на этом результате. Из топологической теоремы Ратнер следует, что семейство ограниченных орбит для эргодического (квази)унипотентного потока $\left(\Gamma \backslash G, g_{\mathbb{R}}\right)$ на некомпактном пространстве не может иметь хаусдорфову размерность, равную $\operatorname{dim} G$. С другой стороны, для элемента вида $g=\gamma\left(\begin{array}{ll}1 & 0 \\ \alpha & 1\end{array}\right) p$, где $\gamma \in S L(2, \mathbb{Z})$ и $p$ - верхнетреугольная матрища, положительная полуорбита $\left\{S L(2, \mathbb{Z}) g A_{t}, t>0\right\}$ геодезического потока ограничена $\Longleftrightarrow$ число $\alpha$ плохо аппроксимируется рациональными числами ([Da86c]). Но множество таких чисел имеет хаусдорфову размерность 1 ([Sch]), и поэтому семейство ограниченных орбит 
геодезического потока имеет полную хаусдорфову размерность (в данном случае 3 ). B [Da86c] Дани распространил этот результат в двух направлениях: либо $G$ - полупростая группа $\mathbb{R}$-ранга 1, либо $G=S L(n, \mathbb{R})$ и $\left\{g_{t}\right\}$ - ее диагональная подгрупша, причем $g_{1}$ имеет всего два (кратных) собственных значения. Этот результат был значительно усилен в [KM96], где, по существу, был доказан следуюший критерий: семейство ограниченных орбит для эргодического потока имеет полную хаусдорфову размерность $\Longleftrightarrow$ максимальное факторпространство $\overline{\Gamma U} \backslash G$ с нулевой энтропией (см. С.3) компактно. Отсюда, в частности, следует существование ограниченных орбит у любого однородного потока на пространстве конечного объема.

Более общий вопрос: верно ли, что эргодический частично-гиперболический поток имеет минимальные множества всех возможных типов, а именно третьего и четвертого, в компактном случае и всех четырех в некомпактном (при наличии $K$-свойства)?

Отметим, что на поверхности бесконечной площади замыкание орициклической орбиты не обязано быть однородным, даже если оно минимально. В самом деле, следуя Хедлунду, нетрудно доказать (cм. [St95b]), что неблуждаюшее множество $\Omega$ орициклического потока $\left(\Gamma \backslash S L(2, \mathbb{R}), u_{\mathbb{R}}\right)$ минимально, если фуксова група $\Gamma$ конечно порождена и не содержит параболических (т.е. унипотентных) элементов (это следует также из работы Бургера $[\mathrm{Bu}])$. Если $Г$ не является равномерной решеткой, то минимальное множество $\Omega$ по нашей классификации имеет второй тип: иначе оно было бы компактным и состояло из одной периодической орбиты (которых нет, так как в $\Gamma$ нет унипотентных элементов). Заметим также, что в данном случае $\Omega$ всюду локально-несвязно.

Наконец, мы укажем на контраст между классом однородных потоков и более обшим классом гладких потоков. Как было отмечено, однородный поток на компактном пространстве может быть минимальньм, лишь если он имеет нулевую энтропию (т.е. является квазиунипотентным). С другой стороны, Эрман $[\mathrm{H}]$ построил минимальный диффеоморфизм на компактном многообразии с положительной энтропией. Далее, согласно теореме 10.10 любое компактное минимальное множество однородного потока локально-связно либо всюду, либо нигде. Первый пример гомеоморфизма плоскости с минимальным множеством, которое локально связно лишь на своем собственном непустом подмножестве, был дан в работе $[\mathrm{Re}]$. Гладкий поток с аналогичньмм минимальньм множеством был построен Джонсоном [Jo] с использованием квазипериодических систем дифференциальных уравнений Миллионшикова [Mi68] и Винограда [Vi].

\section{$\S 11$. Кратное перемешивание и метрическая жесткость однородных потоков}

В этом разделе мы применяем метрическую теорему Ратнер для доказательства гипотезы Маркуса о том, что перемешиваюший однородный поток $\left(\Gamma \backslash G, g_{\mathbb{R}}\right)$ обладает перемешиванием всех кратностей. В связи с одним результатом Рыжикова [Ry91] этот вопрос оказьвается тесно связан с вопросом о мошности множества $\mathscr{M}$ тех моментов времени $t>0$, для который сдвиг $g_{t}$ метрически изоморфен "единичному" сдвигу $g_{1}$. Поэтому нам придется изучить вопрос о жесткости эргодических квазиунипотентных каскадов. Мы покажем, что $\mathscr{M}$ либо не более чем счетно, либо совпадает 
с группой $\mathbb{R}^{+}$.

1. Вначале сформулируем определение кратного перемешивания для действия локально-компактной группы $F$ на лебеговском пространстве $(X, \mu)$.

Действие $F$ на $(X, \mu)$ называется $k$-перемешиваюшим (или принадлежашим классу $\operatorname{Mix}(k))$, если для любых $k+1$ измеримых множеств $A_{0}, \ldots, A_{k} \subset X$ и любых $k+1$ последовательностей $\left\{g_{i}(0)\right\}, \ldots,\left\{g_{i}(k)\right\}$ элементов из $F$ таких, что $g_{i}(l)\left(g_{i}(m)\right)^{-1} \rightarrow \infty, i \rightarrow \infty$, для всех $l \neq m$, имеем

$$
\lim _{i \rightarrow \infty} \mu\left(\bigcap_{l=0}^{k} g_{i}(l) A_{l}\right)=\prod_{l=0}^{k} \mu\left(A_{l}\right) .
$$

Из определения следует, что если действие $F$ на $(X, \mu)$ принадлежит классу $\operatorname{Mix}(k)$, то действие любой замкнутой некомпактной подгрупшы $H \subset F$ на $(X, \mu)$ принадлежит тому же классу.

Старая проблема Рохлина заключается в том, следует ли из однократного перемешивания перемешивание всех высших кратностей, т.е. $\operatorname{Mix}(1) \Rightarrow \operatorname{Mix}(\infty)$ ? В классическом случае $F=\mathbb{Z}, \mathbb{R}$ (т.е. для каскадов и однопараметрических потоков) эта проблема пока решена лиш при весьма сильных ограничениях на действие. Так, Каликов $[\mathrm{Ka}]$ показал, что $\operatorname{Mix}(1) \Rightarrow \operatorname{Mix}(2)$, если поток имеет ранг аппроксимации 1. Недавно этот результат был полностью перекрыт Рыжиковьм [Ry93], который доказал, что проблема Рохлина имеет положительное решение, если поток или автоморфизм имеют конечньй ранг аппроксимации. С другой стороны, имеется пример Ледраппье $[\mathrm{L}] \mathbb{Z}^{2}$-действия класса $\operatorname{Mix}(1)$, но не $\operatorname{Mix}(2)$.

Вернемся в наш класс однородных потоков и рассмотрим проблему Рохлина для геодезического и орициклического потоков на поверхности постоянной отрищательной кривизны конечной плошади. Геодезический поток, будучи аносовским $K$-потоком, обладает перемешиванием любой кратности. В 1962 г. Синай предположил, что и орициклический поток принадлежит классу $\operatorname{Mix}(\infty)$. Эта гипотеза была доказана Маркусом [Mar78]. Более того, он доказал следуюший результат:

TеOPEMA 11.1 ([Mar78]). Пусть $g_{\mathbb{R}}-$ произвольная однопараметрическая подгруппа в полупростой группе Ли $G$ с решеткой $\Gamma \subset G$. Тогда если поток $\left(\Gamma \backslash G, g_{\mathbb{R}}\right)$ перемешивает, то он обладает перемешиванием любой кратности.

Вначале Маркус свел доказательство к случаю унипотентной подгруппы $h_{\mathbb{R}} \subset G$. Скажем, что унипотентная подгруппа $h_{\mathbb{R}}$ в группе Ли $G$ является слабо оришиклической, если найдется такая подгруппа $a_{\mathbb{R}} \subset G$, что

$$
a_{t} h_{1} \alpha_{-t}=h_{e^{t}} \text { для всех } t \in \mathbb{R} .
$$

(Это понятие несколько слабее понятия орицикличности, введенного в $\S 7$, поскольку мы не требуем здесь, чтобы $h_{\mathbb{R}}$ и $a_{\mathbb{R}}$ вкладывались в подгруппу, локально изоморфную $S L(2, \mathbb{R})$; так, например, разрешимая группа Ли может содержать слабо орициклическую подгруппу, но не имеет орициклических подгрупп.) Заметим, что слабо орициклические подгрупшы суть однопараметрические подгруппы орисферических подгрупп. По лемме Морозова-Джекобсона [Ја] любая однопараметрическая унипотентная подгруппа в полупростой группе Ли орициклична. Теперь теорема 11.1 следует из основного результата работы Маркуса: 
TEOPEMa 11.2 ([Mar78]). Пусть $a_{\mathbb{R}}$ и $h_{\mathbb{R}}-$ два непрерывных потока на многообразии $X$ с инвариантной мерой $\mu$. Предположим, что поток $h_{\mathbb{R}}$ әргодичен и $a_{t} \circ h_{1}=h_{e^{t}} \circ a_{t} \partial \Omega_{s}$ всех $t \in \mathbb{R}$. Тогда $h_{\mathbb{R}}$ обладает перемешиванием всех кратностей.

В произвольной групше Ли, однако, унипотентная подгруппа не обязана быть слабо орициклической, даже если она индуцирует перемешиваюший поток (см. пример 11.5). Тем не менее, Маркус предположил, что $\operatorname{Mix}(1) \Rightarrow \operatorname{Mix}(\infty)$ для произвольного однопараметрического потока на однородном пространстве конечного объема (см. также доклад Маргулиса [Ma91b] на Международном конгрессе математиков в Киото, 1990 г.).

Почти одновременно и весьма похожими методами Рыжиков и Мозес получили важные обобшения теоремы 11.2. Во-первых, заметим, что из ее условия вытекает метрический изоморфизм $h_{s} \simeq h_{1}$ для всех $s>0$, причем он устанавливается действием другого потока $a_{\mathbb{R}}$. Имеет место более общий результат:

TеOPEмa $11.3([\mathrm{Ry} 91])$. Пусть $\left(X, h_{\mathbb{R}}\right)$ - сохраняющий меру әргодический поток на пространстве Лебега и $\mathscr{M}=\left\{s \in \mathbb{R} \mid h_{s} \simeq h_{1}\right\}$. Тогда $h_{\mathbb{R}}$ обладает перемешиванием всех кратностей при условии, что М̆ имеет положительную лебеговскую меру.

Естественно возникает вопрос о приложимости данного результата к классу однородных потоков. Довольно легко проблема о кратном перемешивании сводится к унипотентному случаю. Если теперь верно, что множество $\mathscr{M}$ для перемешиваюшего унипотентного потока имеет положительную меру, то гипотеза Маркуса следует из теоремы 11.3. Далее мы, однако, покажем, что даже несчетность множества $\mathscr{M}$ для однородного потока $\left(G / \Gamma, g_{\mathbb{R}}\right)$ влечет за собой слабую орицикличность подгрупш $g_{\mathbb{R}} \subset G$ и поэтому для однородных потоков теоремы 11.2 и 11.3 эквивалентны. Таким образом, гипотеза Маркуса не может быть вьведена из теоремы 11.3.

$\mathrm{C}$ другой стороны, связь между $a_{\mathbb{R}}$ и $h_{\mathbb{R}}$ в теореме 11.2 означает, что на $X$ действует двумерная разрешимая группа с тривиальным центром. Мозес доказал, что $\operatorname{Mix}(1) \Rightarrow$ $\operatorname{Mix}(\infty)$ для всех измеримых действий так назьваемых Ad-собственных групп Ли. Группа Ли $F$ называется Ad-собственной, если центр $Z(F)$ конечен и присоединенная группа $\operatorname{Ad}(F)=F / Z(F)$ замкнута (относительно евклидовой топологии) в алгебраической группе Aut(f) всех автоморфизмов алгебры Ли f.

Teоpema 11.4 ([Moz92,95b]). Пусть Ad-собственная группа Ли F действует сохраняющими меру преобразованиями на лебеговском пространстве $(X, \mu)$. Тогда $\operatorname{Mix}(1) \Rightarrow \operatorname{Mix}(\infty)$.

Таким образом, проблема Рохлина в классе Аd-собственных групп имеет положительное решение. Однако это не помогает нам в решении этой проблемы для однородных потоков.

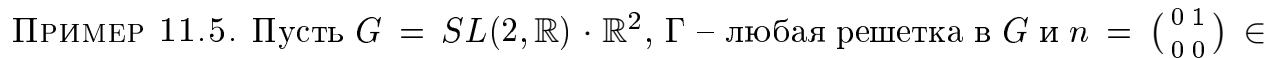
$s l(2, \mathbb{R})$ по отношению к некоторому базису $e_{1}, e_{2}$ в $\mathbb{R}^{2}$. Тогда $\left[n, e_{1}\right]=0,\left[n, e_{2}\right]=e_{1}$. Легко проверить, что элемент $u=n+e_{2}$ не является слабо орищиклическим. В то 
же время унипотентньй поток $(\Gamma \backslash G, \exp (\mathbb{R} u))$ перемешивает, как это следует из критерия Дани C.2. Сушествуют ровно две Ad-собственные подгруппы в $G$, которые содержат $\exp (\mathbb{R} u)$, а именно: $F_{1}=B \cdot \mathbb{R}^{2}$, где $B$ есть двумерная борелевская подгруппа в $S L(2, \mathbb{R})$, содержащая $\exp (\mathbb{R} n)$, и $F_{2}=G$. Однако действие обеих подгрупп не является перемешиваюшим, поскольку действие $\mathbb{R}^{2}$ на $\Gamma \backslash G$ даже не эргодично.

Полностью гипотеза Маркуса была доказана автором.

Tеорема 11.6 ([St93]). Пусть $\left(\Gamma \backslash G, g_{\mathbb{R}}\right)$ - перемешивающий поток на однородном пространстве конечного обгема. Тогда он обладает перемешиванием любой кратности.

Изложим вкратце доказательство этой теоремы. Вначале проведем редукцию к унипотентному случаю. Пусть $\left(\Gamma \backslash G, g_{\mathbb{R}}\right)$ - однопараметрический поток на однородном пространстве конечного объема. Напомним, что с подгруппой $g_{\mathbb{R}} \subset G$ ассоциируется нормальная подгруппа Ауслендера $U \subset G$, порожденная орисферическими относительно $g_{\mathbb{R}}$ подгруппами $G^{+}{ }_{\text {и }} G^{-}$. По теореме С.3 поток $\left(\overline{\Gamma U} \backslash G, g_{\mathbb{R}}\right)$ является максимальньм факторпотоком нулевой энтропии (другими словами, расслоение $\Gamma \backslash G$ на однородные подпространства $\{\overline{\Gamma U} g, g \in G\}$ является разбиением Пинскера) для потока $\left(\Gamma \backslash G, g_{\mathbb{R}}\right)$.

Предположим, что $\left(\Gamma \backslash G, g_{\mathbb{R}}\right)$ перемешивает, где, как всегда, $G$ предполагается связной односвязной группой Ли. Согласно Тувено (см. [Ka]) сохраняюший меру поток обладает перемешиванием любой кратности, если им обладает максимальньй факторпоток нулевой энтропии. Поэтому для доказательства гипотезы Маркуса достаточно доказать, что $\left(\overline{\Gamma U} \backslash G, g_{\mathbb{R}}\right)$ имеет перемешивание всех кратностей, если $\left(\Gamma \backslash G, g_{\mathbb{R}}\right)$ является 1-перемешиваюшим потоком. В силу теоремы Витте С.5, если $\Gamma \backslash G$ допускает перемешивающий поток, то радикал $G$ нильпотентен и решетка $\Gamma$ плотна по Зарисскому в Ad-представлении групшы $G$.

Так как $(\overline{\Gamma U})_{0}$ нормализуется плотной по Зарисскому решеткой $\Gamma$, она является нормальной в $G$. Мы имеем изоморфизм $\left(\overline{\Gamma U} \backslash G, g_{\mathbb{R}}\right) \simeq\left(\Gamma^{\prime} \backslash G^{\prime}, g_{\mathbb{R}}^{\prime}\right)$, где $\Gamma^{\prime}=(\overline{\Gamma U})_{0} \backslash \overline{\Gamma U}$ - решетка в $G^{\prime}=(\overline{\Gamma U})_{0} \backslash \Gamma$ и подгруппа $g_{\mathbb{R}}^{\prime} \subset G^{\prime}$ квазиунипотентна. Поэтому не ограничивая обшности можно изначально считать, что $G=G^{\prime}$ и сама $g_{\mathbb{R}}$ квазиунипотентна.

Так как группа $G$ имеет вид $G=L \cdot N$, ее алгебра Ли является алгебраической, и потому $G$ допускает разложение Жордана для любой однопараметрической подгрупшы. Пусть $g_{t}=c_{t} \times u_{t}, t \in \mathbb{R}$, причем группа $\operatorname{Ad}\left(c_{\mathbb{R}}\right)$ предкомпактна, а група $\operatorname{Ad}\left(u_{\mathbb{R}}\right)$ унипотентна в $\operatorname{Aut}(\mathfrak{g})$. Так как поток $\left(\Gamma \backslash G, c_{\mathbb{R}}\right)$ равномерно непрерьвен, то, следуя Маркусу [Mar78], мы заключаем, что достаточно установить перемешивание всех кратностей для унипотентного потока $\left(\Gamma \backslash G, u_{\mathbb{R}}\right)$.

Теперь мы применим технику джойнингов в проблеме кратного перемешивания. Далее $X=\Gamma \backslash G$ и $\nu$ есть $G$-инвариантная вероятностная мера на $X$, относительно которой унипотентньй поток $\left(\Gamma \backslash G, g_{\mathbb{R}}\right)$ перемешивает. Пусть $\left\{\bar{t}_{i}\right\}=\left\{t_{i}(1), \ldots, t_{i}(k)\right\}$ - набор $k$ последовательностей таких, что $\left|t_{i}(l)-t_{i}(m)\right| \rightarrow \infty$ для всех $l \neq m$. Определим сдвиг диагональной меры $\nu_{\Delta}$ на $X^{k}$ вдоль вектора $\bar{t}_{i}$ формулой

$$
\nu_{\Delta}^{\bar{t}_{i}}\left(\prod_{l=1}^{k} A_{l}\right)=\nu\left(\bigcap_{l=1}^{k} A_{l} g_{t_{i}(l)}\right)
$$


для измеримых $A_{1}, \ldots, A_{k} \subset X$. Заметим, что каждая мера $\nu_{\Delta}^{\bar{t}_{i}}$ на $X^{k}$ является джойнингом $k$ копий потоков $\left(X, g_{\mathbb{R}}\right)$. Ясно, что поток $\left(\Gamma \backslash G, g_{\mathbb{R}}\right)$ является $(k-1)$-перемешиваюшим $\Longleftrightarrow \nu_{\Delta}^{\bar{t}_{i}} \rightarrow \nu^{k}$. Известно, что сходимость джойнингов эквивалентна $*$-слабой сходимости. Так как пространство мер на $X^{k}$, ограниченных условием $\mu\left(X^{k}\right) \leqslant 1$, компактно относительно *-слабой сходимости, то последовательность $\left\{\nu_{\Delta}^{\bar{t}_{i}}\right\}$ имеет хотя бы одну предельную точку $\mu$. Таким образом, чтобы доказать $(k-1)$-перемешивание потока, достаточно установить, что из $\nu_{\Delta}^{\bar{t}_{i}} \rightarrow \mu$ вытекает $\mu=\nu^{k}$.

Пусть $\left(\Gamma \backslash G, g_{\mathbb{R}}\right)$ - перемешиваюший унипотентньй поток. Мы знаем, что $G=L \cdot N$, а по критерию перемешивания С.6 $G=\overline{\Gamma G_{\infty}}$, где $G_{\infty}$ - наименьшая нормальная в $G$ подгруппа, содержашая $L$. Известно [Da77], что $G_{\infty}=L \cdot M$, где $M \subset N$-группа, порожденная произведением $V \subset N$ всех нетривиальных неприводимых $L$-подмодулей в $N$ (здесь мы, по существу, отождествляем нильрадикал $N$ со своей алгеброй Ли).

Обозначим через $p_{l}$ проекцию $G^{k}$ на $l$-ю копию $G$ и через $p_{l}^{*}-$ проекцию $\Gamma^{k} \backslash G^{k}$ на $l$-ю копию $\Gamma \backslash G, l=1, \ldots, k$. Заметим, что $p_{l}^{*}\left(\nu_{\Delta}^{\bar{t}_{i}}\right)=\nu$ и поэтому $p_{l}^{*}(\mu)=\nu$. В частности, $\mu\left(\Gamma^{k} \backslash G^{k}\right)=1$. Ясно, что все меры $\nu_{\Delta}^{\bar{t}_{i}}$ инвариантны относительно диагональной подгрупшы $\left\{\bar{g}_{t}=\left(g_{t}, \ldots, g_{t}\right) \in G^{k}\right\}$ и поэтому предельная мера $\mu$ также $\bar{g}_{\mathbb{R}}$-инвариантна.

Пусть $q: G=L \cdot N \rightarrow L$ и $q^{*}: \Gamma \backslash G \rightarrow q(\Gamma) \backslash L$. Из теоремы Маркуса 11.1, примененной к перемешивающему потоку $\left(q(\Gamma) \backslash L, q\left(g_{\mathbb{R}}\right)\right)$, следует, что $\left(q^{*}\right)^{k} \mu=q^{*}(\nu)^{k}$. Пусть $\mu^{\prime}$ - эргодическая компонента $\mu$ по отношению к унипотентной подгрупе $\bar{g}_{\mathbb{R}} \subset G^{k}$ такая, что $\left(q^{*}\right)^{k} \mu^{\prime}=q^{*}(\nu)^{k}$ и $p_{l}^{*}\left(\mu^{\prime}\right)=\nu$ для всех $l=1, \ldots, k$. Достаточно доказать, что мера $\mu^{\prime}$ является $G^{k}$-инвариантной.

Согласно метрической теореме Ратнер найдутся подгруппа $H \subset G^{k}$ и элемент $g \in G^{k}$ такие, что $\mu^{\prime}$ есть $H$-инвариантная мера, сосредоточенная на замкнутой орбите $\Gamma^{k} g H \subset \Gamma^{k} \backslash G^{k}$. Поскольку $\left(q^{*}\right)^{k} \mu^{\prime}=q^{*}(\nu)^{k}$, то $H=L^{k} \cdot N_{H}$, где $N_{H} \subset N^{k}$. $\mathrm{C}$ другой стороны, $p_{l}^{*}\left(\mu^{\prime}\right)=\nu$ и поэтому $p_{l}\left(N_{H}\right)=N, l=1, \ldots, k$.

Ясно, что если $V^{\prime} \subset V^{k}$ есть такой $L^{k}$-подмодуль, что $p_{l}\left(V^{\prime}\right)=V, l=1, \ldots, k$, то $V^{\prime}=V^{k}$. Следовательно, $V^{k} \subset M^{k} \subset N_{H}$, где $M \subset N$ - нормальная подгруппа в $G$, порожденная $V$. Но из $\overline{\Gamma L M}=G$ вытекает $\overline{\Gamma^{k} L^{k} M^{k}}=G^{k}$ и, так как орбита $\Gamma^{k} g H$ замкнута, $H=G^{k}$ и $\mu^{\prime}=\nu^{k}$.

ЗАмечАнИЕ 1. В доказательстве мы использовали теорему Маркуса 11.1. Однако и этот результат нетрудно доказать алгебраическими методами с помощью теоремы Ратнер.

ЗАмечАние 2. Рыжиков [Ry95] анонсировал общий результат, согласно которому $\operatorname{Mix}(1) \Rightarrow \operatorname{Mix}(\infty)$ для гладкого потока $\varphi_{t}$ на многообразии $M$ с конечной инвариантной гладкой мерой $\mu$ при условии, что автоджойнинги всех степеней являются гладкими мерами на подмногообразиях. Таким образом, гипотеза Маркуса может быть вьведена из теоремы Ратнер без использования теории групп Ли.

ГиПотеЗА. Проблема Рохлина имеет положительное решение для всех однородных действий вида $(\Gamma \backslash G, F)$, где $F$ - замкнутая подгруппа в $G$ и $\Gamma$ - решетка в $G$. 
2. Рассмотрим теперь вопрос о метрической жесткости однородных каскадов. В силу теоремы 5.5 эргодические унипотентные каскады являются метрически жесткими: любой метрический изоморфизм является (почти всюду) аффинным отображением однородных пространств. Мы уже знаем, что частично-гиперболические каскады таким свойством не обладают (см. $\S 2$ ). Что касается квазиунипотентных каскадов и потоков, то, как показьвает пример 10.2, эргодический поток на трехмерном однородном пространстве может быть гладко и метрически изоморфен прямолинейному потоку на трехмерном торе, хотя сами пространства при этом не являются аффинно-эквивалентными. Этот пример хорошо иллюстрирует следуюший результат Ауслендера и Грина:

ТеОРема 11.7 ([AG]). Эргодический квазиунипотентный поток на разрешимом многообразии гладко и метрически изоморфен некоторому әргодическому нильпотоку.

Однако, как доказал Витте, в классе перемешиваюших каскадов имеет место метрическая жесткость:

Tеорема 11.8 ([Wi87]). Метрический изоморфизм перемешивающих однородных квазиунипотентных каскадов (или потоков) является аффинным почти всюду.

В дальнейшем нам потребуется обобщение данной теоремы в классе эргодических квазиунипотентных каскадов. Оказьвается, изоморфизм таких каскадов, хотя и не обязан быть глобально аффинньм отображением, обладает свойством “послойной афифинности":

Tеорема 11.9 ([St95c]). Предположим, что $f:\left(\Gamma \backslash G, g_{\mathbb{Z}}\right) \rightarrow\left(\Gamma^{\prime} \backslash G^{\prime}, g_{\mathbb{Z}}^{\prime}\right)$ есть метрический изоморфизм эргодических квазиунипотентных каскадов. Тогда для почти всех $\Gamma g \in \Gamma \backslash G$ мы имеем $f\left(\overline{\Gamma g G_{\infty}}\right)=\overline{f(\Gamma g) G_{\infty}^{\prime}}$, причем ограничение $f$ на почти каждое однородное подпространство $\overline{\Gamma g G_{\infty}}$ является аффинным.

Как сообщил автору Витте, этот результат также можно получить, используя методы [Wi87] с учетом поправок из [Wi96].

СлЕДСТВИЕ 11.10 ([St95c]). 1) В условиях предыдущей теоремь изоморфизм $f$ индуцирует изоморфизм эргодических факторкаскадов $\hat{f}:\left(\overline{\Gamma G_{\infty}} \backslash G, g_{\mathbb{Z}}\right) \rightarrow$ $\left(\overline{\Gamma^{\prime} G_{\infty}^{\prime}} \backslash G^{\prime}, g_{\mathbb{Z}}^{\prime}\right)$ на разрешимых однородных пространствах.

2) Если каскады являются перемешивающими, то изоморфизм $f$ является аффиннымм (почти всюду).

Заметим, что второе утверждение есть в точности теорема Витте. Оно сразу следует из первого утверждения, поскольку разрешимое однородное пространство не допускает однородных перемешивающих потоков.

Мы приведем лишш идею доказательства теоремы 11.9. Из квазиунипотентности следует, что алгебраическая оболочка подгруппы $\operatorname{Ad}\left(g_{\mathbb{R}}\right) \subset \operatorname{Aut}(\mathfrak{g})$ является прямьм произведением тора $T$ и некоторой унипотентной подгруппы. Следовательно, найдется унипотентная подгруппа $u_{\mathbb{R}} \subset T \cdot G$, которая коммутирует с $T$ и при гладкой 
проекции $G T \rightarrow G$ переходит в $g_{\mathbb{R}}$. Таким образом, мы построили компактное накрытие исходного каскада унипотентным каскадом $\left(\Gamma \backslash G T, u_{\mathbb{Z}}\right)$. Аналогично строится накрытие каскада $\left(\Gamma^{\prime} \backslash G^{\prime}, g_{\mathbb{Z}}^{\prime}\right)$ унипотентным каскадом $\left(\Gamma^{\prime} \backslash G^{\prime} T^{\prime}, u_{\mathbb{Z}}^{\prime}\right)$. График $\operatorname{Graph}(f) \subset \Gamma \backslash G \times \Gamma^{\prime} \backslash G^{\prime}$ компактно накрьвается некоторьм подмножеством $M \subset \Gamma \backslash G T \times \Gamma^{\prime} \backslash G^{\prime} T^{\prime}$, инвариантным относительно диагональной унипотентной подгрупшы $\left(u \times u^{\prime}\right)_{\mathbb{Z}}$. Конечная эргодическая относительно $\left(g \times g^{\prime}\right)_{\mathbb{Z}}$ мера на $\operatorname{Graph}(f)$ (получаемая переносом $G$-инвариантной меры на $\Gamma \backslash G$ ) накрывается некоторой конеч-

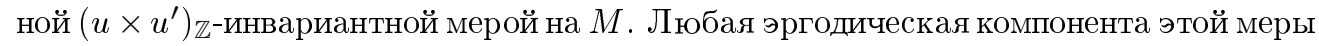
имеет алгебраическое происхождение и накрьвает эргодическую меру на $\operatorname{Graph}(f)$. Таким образом, Graph $(f)$ компактно накрывается однородным пространством. Наконец, равенства $(G T)_{\infty}=G_{\infty},\left(G^{\prime} T^{\prime}\right)_{\infty}=G_{\infty}^{\prime}$ позволяют доказать, что $\operatorname{Graph}(f)$, хотя и не обязан быть однородным подпространством, расслаивается на однородные подпространства вида $\operatorname{Graph}\left(\left.f\right|_{\overline{\Gamma g G_{\infty}}}\right) \subset \Gamma \backslash G, g \in G$.

3. Теперь для однородного потока $\left(\Gamma \backslash G, g_{\mathbb{R}}\right)$ мы готовы обсудить структуру множества $\mathscr{M}=\left\{t>0: g_{t} \simeq g_{1}\right\}$. Ясно, что если поток частично-гиперболичен, то множество $\mathscr{M}$ состоит только из единичного элемента $1 \in \mathbb{R}$ (это следует из соотношения для энтропии $\left.e\left(g_{t}\right)=t e\left(g_{1}\right), t>0\right)$. Таким образом, множество $\mathscr{M}$ может быть нетривиально лишш для квазиунипотентных потоков.

Рассмотрим конкретный пример, когда множество $\mathscr{M}$ нетривиально. Возьмем двумерньй тор $T^{2}=\mathbb{Z}^{2} \backslash \mathbb{R}^{2}, A \in S L(2, \mathbb{Z})$ и $v \in \mathbb{R}^{2}$. Тогда прямолинейные потоки $\left(T^{2}, \mathbb{R} v\right)$ и $\left(T^{2}, \mathbb{R} A(v)\right)$ изоморфны. В частности, если вектор $v \in \mathbb{R}^{2}$ является собственным, т.е. $A(v)=\lambda v$ для некоторого $\lambda>0$, то каскады $\left(T^{2}, \mathbb{Z} v\right)$ и $\left(T^{2}, \lambda \mathbb{Z} v\right)$ изоморфны. Таким образом, множество $\mathscr{M}$ содержит мультипликативную циклическую группу $\lambda^{\mathbb{Z}} \subset \mathbb{R}^{+}$. Нетрудно показать, используя свойство метрической жесткости таких каскадов, установленное еще Перри [Р71], что в данном случае множество $\mathscr{M}$ является счетньм.

$\mathrm{C}$ другой стороны, по определению для слабо орициклической подгрупшы $u_{\mathbb{R}} \subset G$ имеем $\mathscr{M}=\mathbb{R}^{+}$. Оказьвается, других вариантов и не бьвает:

ТеОрема 11.11 ([St95c]). Предположим, что множество $\mathscr{M}=\{t>0 \mid$ $\left.u_{t} \simeq u_{1}\right\}$ для эргодического однородного потока $\left(\Gamma \backslash G, u_{\mathbb{R}}\right)$ на пространстве конечного обвема несчетно. Тогда подгруппа $u_{\mathbb{R}}$ слабо оричиклична в $G$ ( и поэтому $\mathscr{M}=\mathbb{R}^{+}$).

Покажем вначале, как эта теорема доказьвается для унипотентных потоков. Не ограничивая общности мы будем предполагать, что решетка $\Gamma \subset G$ несократима. Предположим, что найдется несчетное множество $\mathscr{M} \subset \mathbb{R}^{+}$такое, что для всякого $t \in \mathscr{M}$ существует метрический изоморфизм эргодических унипотентных каскадов $\varphi_{t}:\left(\Gamma \backslash G, u_{\mathbb{Z}}\right) \rightarrow\left(\Gamma \backslash G, u_{t \mathbb{Z}}\right)$. В силу метрической жесткости для каждого $t \in \mathscr{M}$ найдутся элемент $c(t) \in G$ и автоморфизм $\sigma_{t} \in \operatorname{Aut}(G, \Gamma)$ такие, что почти всюду на $\Gamma \backslash G$ изоморфизм $\varphi_{t}$ имеет вид $\varphi_{t}(\Gamma g)=\Gamma \sigma_{t}(g) c(t)$. Так как ввиду плотности $\Gamma$ в Ad-представлении группа $\operatorname{Aut}(G, \Gamma)$ не более чем счетна, то для некоторого несчетного подмножества $\mathscr{M}^{\prime} \subset \mathscr{M}$ автоморфизмы $\sigma_{t}$ совпадают с некоторьм фиксированным автоморфизмом $\sigma \in \operatorname{Aut}(G, \Gamma)$. Поскольку $\varphi_{t}$ переводит правое действие подгрупшы $u_{\mathbb{Z}}$ в правое действие $u_{t \mathbb{Z}}$, то $\varphi_{t}\left(\Gamma g u_{1}\right)=\varphi_{t}(\Gamma g) u_{t}$ почти всюду на $\Gamma \backslash G$. 
Используя явньй вид $\varphi_{t}, t \in \mathscr{M}^{\prime}$, мы убеждаемся, что $\Gamma \sigma(g) \sigma\left(u_{1}\right) c(t)=\Gamma \sigma(g) c(t) u_{t}$ для почти всех $g \in G$. Но тогда элемент $\sigma\left(u_{1}\right) c(t) u_{-t} c(t)^{-1}$ имеет тривиальное действие на $\Gamma \backslash G$ и потому обязан лежать в пересечении центра $Z(G)$ и решетки $\Gamma$. Ввиду несократимости $\Gamma$ такое пересечение тривиально и поэтому $c(t) u_{t} c(t)^{-1}=\sigma\left(u_{1}\right)$ для каждого $t \in \mathscr{M}^{\prime}$. Зафиксируем произвольный элемент $s \in \mathscr{M}^{\prime}$ и заметим, что $c(t)^{-1} c(s) u_{s} c(s)^{-1} c(t)=u_{t}$ для каждого $t \in \mathscr{M}^{\prime}$. Теперь наша теорема следует из следующего несложного замечания:

Лемма 11.12 ([St95c]). Предположим, что $u_{\mathbb{R}}-$ такая подгруппа в $G$, что для каждого элемента $t$ из некоторого несчетного множества $\mathscr{M}^{\prime} \subset \mathbb{R}^{+}$найдется $h(t) \in G$ такой, что $h(t) u_{1} h(t)^{-1}=u_{t}$. Тогда подгруппа $u_{\mathbb{R}}$ слабо оричиклична.

Рассмотрим, наконец, эргодический квазиунипотентный каскад $\left(\Gamma \backslash G, u_{\mathbb{Z}}\right)$ с несчетным множеством $\mathscr{M}=\left\{t>0 \mid u_{t} \simeq u_{1}\right\}$. В силу следствия 11.10 для любого $t \in \mathscr{M}$ факторкаскад $\left(\overline{\Gamma G_{\infty}} \backslash G, u_{t \mathbb{Z}}\right)$ изоморфен факторкаскаду $\left(\overline{\Gamma G_{\infty}} \backslash G, u_{\mathbb{Z}}\right)$. Ввиду 11.7 эргодический квазиунипотентньй поток на разрешимом пространстве гладко и метрически изоморфен некоторому нильпотоку. Мы уже доказали теорему 11.11 в унипотентном случае и можем утверждать, что множество $\mathscr{M}$ для произвольного нильпотока всегда не более чем счетно, поскольку нильпотентная группа Ли не содержит слабо орициклических подгрупп. Следовательно, пространство $\overline{\Gamma G_{\infty}} \backslash G$ вырождается в точку, и по теореме С.6 любой эргодический каскад на $\Gamma \backslash G$ перемешивает. Поэтому мы можем применить теорему 11.8 Витте и заключить, что любой метрический изоморфизм $u_{t} \simeq u_{1}, t \in \mathscr{M}$, задается аффиинньм отображением. Дальнейшие рассуждения дословно повторяют схему доказательства в унипотентном случае.

\section{§ 12. Эргодические меры и замыкания орбит для действия произвольных подгрупп}

1. Мы знаем, что для связных подгрупп $H \subset G$, порожденных унипотентными элементами, конечные эргодические меры и замыкания орбит на $\Gamma \backslash G$ (при условии $\operatorname{vol}(\Gamma \backslash G)<\infty)$ имеют алгебраическое происхождение. Если $H$ порождена квазиунипотентными элементами, по аналогии с леммой 10.3 нетрудно построить компактное расширение $G^{*}$ группы $G$, в котором наша подгруппа $H$ лежит в компактном расширении связной подгруппы $U \subset G^{*}$, порожденной унипотентными элементами. Отсюда легко вывести, что любая конечная $H$-эргодическая мера является "хорошей” : она сосредоточена на гладком подмногообразии в $\Gamma \backslash G$ и на своем носителе является гладкой мерой. Аналогично, при условии конечности объема $\Gamma \backslash G$ замыкания $H$-орбит являются гладкими (и даже аналитическими) подмногообразиями в $\Gamma \backslash G$.

Предположим теперь, что связная подгруппа $H$ вообще не содержит (квази)унипотентных элементов. Разумеется, при $\operatorname{dim}(H)=1$ (т.е. для частично-гиперболического потока) нельзя гарантировать, что все эргодические меры и замыкания орбит являются сколь-нибудь хорошими. Однако для достаточно больших подгрупп $H \subset G$ дела могут обстоять значительно лучше. Пусть, например, $G$-полупростая группа Ли и $H$ - ее максимальная $\mathbb{R}$-диагонализуемая подгруппа, причем $\operatorname{dim}(H)=\operatorname{rank}_{\mathbb{R}}(G) \geqslant 2$. Тогда, взяв неприводимую решетку $\Gamma \subset G$, можно надеяться, что эргодические меры и замыкания $H$-орбит имеют алгебраическое происхождение. Однако, как показывает пример Рис, при этом нужна некая осторожность. В этом примере строится 
равномерная решетка $\Gamma \subset G=S L(3, \mathbb{R})$, которая пересекает по решетке две коммутирующие подгрупшы $L, D \subset G$, причем $L$ изоморфна $S L(2, \mathbb{R})$, а $D$ одномерна и $\mathbb{R}$-диагонализуема. Ясно, что, взяв диагональную подгруппу $D_{L} \subset L$ и помножив ее на $D$, мы получим максимальную $\mathbb{R}$-диагонализуемую подгруппу в $G$, и что любая “плохая" $D_{L}$-эргодическая мера для геодезического потока $\left((\Gamma \cap L) \backslash L, D_{L}\right)$ дает “плохую” $D_{L} \times D$-эргодическую меру для двумерного потока на $\Gamma \backslash G$. То же относится и к замыканиям орбит. Эти соображения привели Катка и Спатциера [KS96] к следующей гипотезе. Если $H$ - максимальная $\mathbb{R}$-диагонализуемая подгруппа в полупростой группе Ли $G$ и $\Gamma$ - решетка в $G$, то конечная $H$-эргодическая мера $\mu$ на $\Gamma \backslash G$ либо алгебраична, либо имеет следуюшее происхождение: $H$-действие на некотором инвариантном замкнутом однородном подпространстве проектируется на одномерный факторпоток и мера $\mu$ получается поднятием эргодической меры этого факторпотока. С использованием метрической теоремы Ратнер в [KS96] Каток и Спатциер при некоторых предположениях доказали алгебраичность $H$-эргодических мер положительной энтропии, однако в общем случае эта проблема остается открытой.

Заметим, что для неравномерной решетки $\Gamma=S L(3, \mathbb{Z}) \subset G=S L(3, \mathbb{R})$ гипотеза об алгебраичности замыканий орбит диагональной подгрупшы $H \subset G$ не опровергнута. Более того, как замечено Маргулисом (а в неявном виде и в $[\mathrm{CS}]$ ), у нее имеются сильные приложения в теории чисел. А именно, если установить компактность всех ограниченных $H$-орбит в $\Gamma \backslash G$, будет доказана известная гипотеза Литлвуда: $\liminf _{n \rightarrow \infty} n\{n \alpha\}\{n \beta\}=0$ для любых $\alpha, \beta \in \mathbb{R}$ (здесь $\{x\}$ обозначает дробную часть $x \in \mathbb{R})$.

2. Рассмотрим теперь “смешанньй” случай: $H$ порождена унипотентными и $\mathbb{R}$-диагонализуемыми элементами (см. также [R94,95b], [B]). Простейший случай: $H=A U$, где одномерная $\mathbb{R}$-диагонализуемая подгруппа $A$ является “диагональной” по отношению к унипотентной подгрупе $U$ (см. $\S 7$ ). Тогда согласно результатам Ратнер (см. теорему 7.4) любая конечная $H$-эргодическая мера $\mu$ является инвариантной (и, разумеется, эргодической) относительно подгрупшы $S L_{2}(U, A) \subset G$, содержащей $H$ и локально-изоморфной $S L(2, \mathbb{R})$. Так как $S L_{2}(U, A)$ уже порождена унипотентными элементами, проблема отпадает: мера $\mu$ является алгебраической.

Это подсказьвает следуюшее обобщение:

ТЕОРема 12.1 ([R94]). Предположим, что связная подгруппа $U \subset G$ порождена унипотентными элементами и подгруппь $A_{1}, \ldots, A_{n} \subset G$ являются "диагональными" для унипотентных подгрупп $U_{1}, \ldots, U_{n} \subset U$ соответственно. Пусть $H \subset G$ порождена $U$ и всеми $A_{1}, \ldots, A_{n}$. Тогда каждая конечная $H$-эргодическая мера на $\Gamma \backslash G$ является алгебраической. Если $\operatorname{vol}(\Gamma \backslash G)<\infty$, то все замыкания $H$-орбит на $\Gamma \backslash G$ являются однородными подпространствами в $\Gamma \backslash G$ конечного обгема.

Разумеется, в данной теореме эргодические меры и замыкания орбит для $H$ совпадают с эргодическими мерами и замыканиями орбит для подгрупшы $H^{\prime} \subset G$, порожденной $U$ и всеми $S L_{2}\left(U_{i}, A_{i}\right)$.

В частности, условия теоремы вьполнены, если подгруппа $H \subset G$ параболична в полупростой групше $G_{1} \subset G$ без компактных множителей.

Метрическая часть теоремы 12.1 была усилена Мозесом следующим образом. По 
Бьену и Борелю [BВ] подгруппа $H \subset G$ называется эпиморфной в $G$, если $H$-неподвижные векторы в любом конечномерном представлении группы $G$ являются и $G$-неподвижными. Известно [Rag], что параболическая подгруппа в полупростой группе Ли без компактных множителей является эпиморфной. В частности, каждая подгруппа $A_{i} U_{i}$ эпиморфна в $S L_{2}\left(U_{i}, A_{i}\right)$. Отсюда следует, что $H$ из теоремы 12.1 эпиморфна по отношению к группе $H^{\prime} \subset G$, порожденной всеми $S L_{2}\left(U_{i}, A_{i}\right)$. Поэтому следуюший результат Мозеса действительно усиливает теорему 12.1 .

TeOpema 12.2 ([Moz95a]). Пусть подгруппа $H \subset G$ эпиморфна по отношению к подгруппе $H^{\prime} \subset G$, порожденной связными унипотентными подгруппами. Тогда каждая конечная $H$-әргодическая мера на пространстве $\Gamma \backslash G$ алгебраична.

Аналог данной теоремы для замыканий орбит пока не доказан. Любопытно, что согласно $[\mathrm{BB}]$ в каждой простой некомпактной группе Ли $G, \operatorname{dim} G>3$, можно найти 3 -мерную разрешимую эпиморфную подгруппу. Ясно, что эта подгруппа строго эргодически действует на любом факторпространстве $G$ конечного объема.

Предположим теперь, что подгруппа $H \subset G$ порождена элементами $g \in G$, у которых все собственные числа оператора $\operatorname{Ad}_{g}$ вешественны и положительны. Обозначим через $H_{u} \subset H$ нормальную подгруппу, порожденную всеми унипотентными однопараметрическими подгруппами в $H$. С помощью следующего результата Маргулиса и Томанова изучение $H$-эргодических мер на $\Gamma \backslash G$ (где подгруппа $\Gamma \subset G$ дискретна) может быть сведено к случаю, когда подгруппа $H_{u}$ тривиальна. Рассмотрим разложение $H$-эргодической инвариантной вероятностной меры $\mu$ на алгебраические $H_{u}$-эргодические компоненты. Имеет место

Теорема 12.3 ([МТ96]). В указанной ситуации найдется связная замкнутая подгруппа $L \subset G$ такая, что почти каждая $H_{u}$-әргодическая компонента является $L$-инвариантной мерой на замкнутой $L$-орбите. Eсли $P \subset G-$ группа всех әлементов в $G$, которые нормализуют $L$ и сохраняют на ней меру Хаара, то $H \subset P$ и $\mu(x P)=1$ для некоторой точки $x \in \Gamma \backslash G$.

Так как подгрупша $L$ нормальна в $P$, пространство $X$ разбиения $x P$ на $L$-орбиты является однородным $P / L$-пространством. Мера $\mu$, будучи $L$-инвариантной, проектируется на $A$-инвариантную эргодическую меру $\mu^{\prime}$ на $X$, где $A=H L / L$. Ясно, что $\mu^{\prime}$ алгебраична $\Longleftrightarrow \mu$ алгебраична (заметим, что группа $A$ абелева, если $H$ связна). В [МТ96] также показано, как теорема Мозеса 12.2 выводится из теоремы 12.3. Заметим, что в [МТ96] рассматривается намного более общая ситуация $(G-$ произведение групп Ли над локальными полями, $\Gamma$ - любая ее замкнутая подгруппа и т. д.). $\mathrm{C}$ другой стороны, в $p$-адическом случае на $H \subset G$ накладывается дополнительное ограничение (необязательное для вешественных групп Ли).

Приведем результат Шаха [Sh96], также имеюший отношение к действиям произвольных подгрупп. Допустим, что $G_{1}$ - полупростая подгруппа без компактных множителей в группе Ли $G, a \in G_{1}$ - полупростой элемент и $U \subset G_{1}$ - расширяюшаяся подгруппа в $G_{1}$ для элемента $a$ (т.е. $U=G^{+} \cap G_{1}$, где $G^{+}$- расширяюшаяся орисферическая подгруппа в $G$ ). Пусть $\Gamma$ - решетка в $G$ и $\pi: G \rightarrow \Gamma \backslash G$-естественная 
проекция. Предположим без ограничения общности, что $U$ не содержится в собственной нормальной подгрупше группы $G_{1}$ и что $\overline{\Gamma G_{1}}=\Gamma \backslash G$. Рассмотрим любую площадку $B \subset U$ с конечной положительной мерой Хаара $m$ на $U$. При $n \rightarrow+\infty$ сдвиг $\Gamma B a^{n}=\Gamma a^{n}\left(a^{-n} B a^{n}\right)$ растягивает площадку $B$. Оказывается, эта последовательность площадок становится все более и более равномерно распределенной в пространстве конечного объема $\Gamma \backslash G$. Точнее, если $\lambda=\left.m\right|_{B}$ - ограничение меры Хаара с $U$ на $B$, то последовательность конечных мер $a^{n} \pi(\lambda)$ на $\Gamma \backslash G$ стремится к $G$-инвариантной мере в $*$-слабой топологии. Для $G=G_{1}$ этот факт был хорошо известен и ранее, но в обшем случае результат является весьма нетривиальным. Разумеется, из него следует, что подгруппа $a^{\mathbb{Z}} U$ имеет плотную орбиту точки $\Gamma \in \Gamma \backslash G$ (впрочем, этот же факт следует из теоремы 12.1).

3. Заметим, что приведенные результаты позволяют решить вопрос о строении эргодических мер и замьканий орбит для действия связных подгрупп $H \subset G$ на пространстве конечного объема $\Gamma \backslash G$, если $G$ является полупростой группой Ли $\mathbb{R}-$ ранга 1. Обозначим через $A \subset G$ максимальную $\mathbb{R}$-диагонализуемую подгрупу, $\operatorname{dim}(A)=1$, и через $U \subset G$ - расширяюшуюся орисферическую подгруппу для $A$. Пусть также $K_{1} \subset G$ - компактная компонента централизатора $A$. Тогда $P=\left(K_{1} \times A\right) \cdot U-$ параболическая подгруппа в $G$. Если связная замкнутая подгруппа $H \subset G$ не порождается квазиунипотентными элементами, то она сопряжена подгруппе в $P$. Ясно, что из некомпактных подгрупп в $P$ лишь абелевы редуктивные подгрупшы могут иметь (и имеют) "плохие" эргодические меры и замыкания орбит (это можно вывести как из теоремы Ратнер 12.1, так и из приведенного результата Шаха). Таким образом, критерий здесь вьглядит следуюшим образом: если $H \subset G$ - некомпактная связная подгрупша, то на $\Gamma \backslash G$ найдется "плохая" $H$-эргодическая мера $\Longleftrightarrow$ группа $H$ сопряжена подгруппе в $K_{1} \times A$. Аналогичное утверждение имеет место и для замыканий орбит.

Подобная задача для разрешимых групп Ли еше не решена. Один из наиболее интересных случаев здесь связан с изучением орбит и эргодических мер для пары эргодических автоморфизмов тора, один из которых является аносовским. Когда среди фактор-действий имеется почти циклическая група эргодических автоморфизмов тора, для исходного действия естественно возникают "плохие" эргодические меры и замькания орбит. Если же таких фактор-действий не имеется, то все минимальные множества конечны (Беренд, $[\mathrm{Be}])$ и это позволяет надеяться на хорошее поведение всех орбит. Интересные результаты об алгебраичности эргодических мер для таких действий получены Катком и Спатциером [KS96].

\section{$\S 13$. Унипотентные потоки на однородных пространствах над локальными полями}

1. Гипотеза Опенгейма-Давенпорта о значениях квадратичных форм в целых точках имеет смысл не только для поля вещественных чисел, но и в так называемом $S$-арифметическом контексте (см. §14). Это наводит на естественную мысль о справедливости гипотез Рагунатана и Дани над любым локальньм (недискретньм локально-компактным) полем $k$ нулевой характеристики. Более того, можно рассмотреть действие унипотентных потоков на однородных пространствах вида $\Gamma \backslash H$, где $H-$ 
замкнутая подгрупша в произведении $G=\prod_{i=1}^{n} G_{i}$ групп Ли $G_{i}$ над (различными) локальными полями $k_{i}$ нулевой характеристики, а $\Gamma \subset H$ - дискретная подгрупа. Полностью обе гипотезы в такой постановке были доказаны Ратнер [R95a], а метрическая - также Маргулисом и Томановьг [MT94,96]. Заметим, что в [MT94] был рассмотрен случай, когда все групшы $G_{i}$ алгебраичны; в [MТ96] это ограничение снято (и к тому же замкнутая подгруппа $\Gamma \subset H$ не предполагается дискретной). Способ доказательства Ратнер состоит в том, что вначале развивается теория групп Ли над полем $\mathbb{Q}_{p} p$-адических чисел, а затем доказательство для $k=\mathbb{R}$ прослеживается с тем, чтобы внести необходимые изменения в общем случае. Способ Маргулиса и Томанова (помимо некоторых идей Ратнер) интенсивно использует теорию алгебраических групп и энтропийную теорию и дает новое доказательство метрической теоремы даже для вешественного случая. Мы не будем вдаваться в подробности доказательства в обшем случае, но остановимся на новых моментах, которые появляются при рассмотрении $p$-адического случая, следуя работе Ратнер [R95a] (см. также [B], [R95b]).

2. Известно $[\mathrm{VdV}]$, что локальное поле нулевой характеристики есть либо $\mathbb{R}$, либо $\mathbb{C}$, либо конечное расширение поля $p$-адических чисел $\mathbb{Q}_{p}$ (при некотором простом $p \in \mathbb{N}$ ). Поэтому можно предполагать, что $G=\prod_{i=1}^{n} G_{i}$, где $S=\left\{p_{1}, \ldots, p_{n}\right\}$ - некоторое конечное множество различных простых чисел (включая, быть может, $\infty$ ), а $G_{i}$ - группа Ли над полем $\mathbb{Q}_{p_{i}}, p_{i} \in S$ (либо над $\mathbb{R}$, если $p_{i}=\infty$ ). Разумеется, в значительной мере задача сводится к рассмотрению однородных пространств группы Ли над $\mathbb{Q}_{p}$.

Напомним основные факты из теории $p$-адических чисел (всюду далее $p$ - простое число). Каждый элемент $x \in \mathbb{Q}$ единственным образом представляется в виде $x=p^{n} a / b$, где $n, a \in \mathbb{Z}, b \in \mathbb{N}$, причем $p, a, b$ взаимно просты. Поле $\mathbb{Q}_{p}$ является пополнением $\mathbb{Q}$ по метрике $|x|_{p}=p^{-n}$. Известно, что поля $\mathbb{Q}_{p}$ и $\mathbb{Q}_{s}$ неизоморфны при $p \neq s$. Кроме того, $\mathbb{Q}_{p}$ ни при каком конечном $p$ не изоморфно $\mathbb{R}$.

Пополнением кольца $\mathbb{Z} \subset \mathbb{Q}$ является кольцо цельх $p$-адических чисел $\mathbb{Z}_{p} \subset \mathbb{Q}_{p}$. Известно, что $\mathbb{Z}_{p}$ является компактной открытой (аддитивной) подгруппой в $\mathbb{Q}_{p}$ и имеет мощность континуума. Число $\alpha \in \mathbb{Z}_{p}$ назьвается $p$-адической единицей, если найдется $\beta \in \mathbb{Z}_{p}$ такое, что $\alpha \beta=1$. Множество $p$-адических единиц образует компактную мультипликативную групу $O_{p}$. В $O_{p}$ выделяется подгруппа $1+p \mathbb{Z}_{p}$ главных единиц. Легко проверить, что $O_{p}=\left\{\mathbb{Z}_{p}\right\}-\left\{p \mathbb{Z}_{p}\right\}$.

Ясно, что в $\mathbb{Z}_{p}$ и $\mathbb{Q}_{p}$ нет дискретных аддитивных подгрупп. ${ }^{14}$ Заметим, что $\mathbb{Q}_{p}$ является пределом возрастающей последовательности компактных подгрупп $\left\{p^{-n} \mathbb{Z}_{p}\right.$, $n \in \mathbb{N}\}$ и потому любая собственная замкнутая подгруппа $H \subset \mathbb{Q}_{p}$ компактна. Любая циклическая группа вида $\alpha^{\mathbb{Z}}$ при $|\alpha|_{p} \neq 1$ дискретна в мультипликативной групе $\mathbb{Q}_{p}^{*}=\mathbb{Q}_{p}-\{0\}$. Максимальная дискретная подгруппа $\Sigma_{p} \subset O_{p}$ конечна и состоит из корней 1. Известно (cм. [Se82]), что при $p>2$ группа $\Sigma_{p}$ является циклической порядка $p-1$, причем $\Sigma_{p} \cap\left(1+p \mathbb{Z}_{p}\right)=1$ и $O_{p}=\Sigma_{p} \times\left(1+p \mathbb{Z}_{p}\right)$. Если $p=2$, то $\Sigma_{p}= \pm 1$.

\footnotetext{
${ }^{14}$ Заметим однако, что в $\mathbb{R} \times \mathbb{Q} p$ такие подгруппы есть: например, группа рациональных чисел вида $m / p^{k}, m \in \mathbb{Z}, k \in \mathbb{N}$, диагонально погруженная в $\mathbb{Q} \times \mathbb{Q} \subset \mathbb{R} \times \mathbb{Q}_{p}$. Легко убедиться, что эта подгруппа является бесконечно-порожденной равномерной решеткой в $\mathbb{R} \times \mathbb{Q}_{p}$.
} 
Рассмотрим ряд

$$
\exp (x)=\sum_{i=0}^{\infty} \frac{x^{i}}{i !}
$$

Оценив норму $\left|\frac{x^{i}}{i !}\right|_{p}$, можно убедиться, что этот ряд сходится для каждого $x \in p \mathbb{Z}_{p}$ при $p>2$ и для каждого $x \in 4 \mathbb{Z}_{2}$ при $p=2$. Таким образом, при $p>2$ мы имеем непрерьвный гомоморфизм ехр: $p \mathbb{Z}_{p} \rightarrow 1+p \mathbb{Z}_{p}$, а при $p=2-$ непрерьвный гомоморфизм $\exp : 4 \mathbb{Z}_{2} \rightarrow 1+4 \mathbb{Z}_{2}$. Гораздо проше проверить, что формула

$$
\ln (x)=\sum_{i=1}^{\infty}(-1)^{i+1} \frac{(x-1)^{i}}{i}
$$

задает непрерьвный гомоморфизм $\ln : 1+p \mathbb{Z}_{p} \rightarrow p \mathbb{Z}_{p}$ при любом $p$. Так как ехр и $\ln$ взаимно обратны в кольце формальных степенных рядов, мы вьводим, что если $p>2$, то exp и $\ln$ являются взаимно обратньми изоморфизмами. При $p=2$ ядро $\ln$ содержит $\Sigma_{2}= \pm 1$; поэтому ехр и $\ln$ являются изоморфизмами, если их определить на $4 \mathbb{Z}_{2}$ и $1+4 \mathbb{Z}_{2}$ соответственно.

Отсюда следует, что при $p>2$ любая замкнутая подгруппа в $1+p \mathbb{Z}_{p}$ имеет вид $1+p^{k} \mathbb{Z}_{p}$ для некоторого целого $k \geqslant 1$ (аналогично, любая замкнутая подгруппа в $1+4 \mathbb{Z}_{2}$ имеет вид $1+2^{k} \mathbb{Z}_{2}$ для некоторого целого $k \geqslant 2$ ). Следовательно, предел любой возрастаюшей последовательности компактных подгруп в $O_{p}$ компактен (при любом $p$ ).

Непрерьвный гомоморфизм груп $\varphi: \mathbb{Z}_{p} \rightarrow 1+p \mathbb{Z}_{p}$ можно получить, взяв любой элемент $\alpha$ в $1+p \mathbb{Z}_{p}$ (или в $1+4 \mathbb{Z}_{2}$, если $p=2$ ) и определив $\varphi(x)=\alpha^{x}=\exp (x \ln (\alpha))$. $\mathrm{C}$ другой стороны, как легко убедиться, любой непрерьвный гомоморфизм $\psi: \mathbb{Q}_{p} \rightarrow$ $\mathbb{Q}_{p}^{*}$ тривиален. В то же время, так как $\mathbb{Q}_{p}^{*}=p^{\mathbb{Z}} O_{p}=p^{\mathbb{Z}} \Sigma_{p}\left(1+p \mathbb{Z}_{p}\right)$, мы можем доопределить $\ln :\left(1+p \mathbb{Z}_{p}\right) \rightarrow p \mathbb{Z}_{p}$ тривиальным образом на $p^{\mathbb{Z}} \Sigma_{p}$ (или на $p^{\mathbb{Z}}$, если $p=2$ ) и получить непрерьвньй гомоморфизм $\psi: \mathbb{Q}_{p}^{*} \rightarrow \mathbb{Q}_{p}$. Нелишне заметить, что образ $\psi\left(\mathbb{Q}_{p}^{*}\right) \subset \mathbb{Q}_{p}$ всегда предкомпактен, так как $O_{p}-$ компакт и $\psi\left(p^{\mathbb{Z}}\right) \subset\left\{\alpha \in \mathbb{Q}_{p}\right.$ : $\left.|\alpha|_{p} \leqslant|\psi(p)|_{p}\right\}$.

3. Перейдем теперь к рассмотрению груп Ли над $\mathbb{Q}_{p}$. Упрощенно говоря, группа $G$ есть $n$-мерная группа Ли над $\mathbb{Q}_{p}$, если она допускает атлас из карт, гомеоморфных $\mathbb{Z}_{p}^{n}$ (напомним, что $\mathbb{Z}_{p}$ является компактной окрестностью 0 в $\mathbb{Q}_{p}$ ), причем сквозные отображения и груповые операции в этом атласе аналитичны (точное определение см. у Ceppa [Se69] или в [DSMS]). Замкнутая подгруппа группы Ли над $\mathbb{Q}_{p}$ сама является группой Ли над $\mathbb{Q}_{p}[\mathrm{Se} 69]$. Ясно, что $\mathbb{Z}_{p}, \mathbb{Q}_{p}$ и $\mathbb{Q}_{p}^{*}-$ групшы Ли над $\mathbb{Q}_{p}$. Наиболее важным многомерным примером является група $G L\left(n, \mathbb{Q}_{p}\right) n$-мерных матриц с коэффициентами из $\mathbb{Q}_{p}$ и ненулевым определителем. В этой групе выделяется подгруппа $G L\left(n, \mathbb{Z}_{p}\right)$ матриц $A$ с цельми коэффициентами и условием $|\operatorname{det}(A)|_{p}=1$. Известно [Se69], что $G L\left(n, \mathbb{Z}_{p}\right)$ является максимальной (открытой) компактной подгруппой в $G L\left(n, \mathbb{Q}_{p}\right)$ и любая максимальная компактная подгруппа в $G L\left(n, \mathbb{Q}_{p}\right)$ сопряжена с $G L\left(n, \mathbb{Z}_{p}\right)$. Известно также, что в $G L\left(n, \mathbb{Z}_{p}\right)$ имеется открытая подгруппа $V$, не содержашая конечных подгрупп (при $n=1, p>2$ таковаподгруппа $V=1+p \mathbb{Z}_{p} \subset O_{p}=$ $G L\left(1, \mathbb{Z}_{p}\right)$, а при $n=1, p=2$ - подгрупп $\left.V=1+4 \mathbb{Z}_{2}\right)$. Следовательно, порядок любой конечной подгруппы из $G L\left(n, \mathbb{Q}_{p}\right)$ ограничен числом элементов в $G L\left(n, \mathbb{Z}_{p}\right) / V$. 
Далее наше изложение следует [R95a]. Пусть $G$ - произвольная группа Ли над $\mathbb{Q}_{p}$ с алгеброй Ли $\mathfrak{g}$. Выделим в $\mathfrak{g}$ любой $n$-мерный базис и положим $\|v\|=\max _{i}\left|v_{i}\right|_{p}$ для каждого вектора $v \in \mathfrak{g}$. Для $\varepsilon>0$ определим векторное подпространство $\mathfrak{g}_{\varepsilon}(0)=$ $\{v \in \mathfrak{g}:\|v\|<\varepsilon\}$ над $\mathbb{Z}_{p}$. Известно [Sе69], что имеется $\delta>0$ такое, что $\left[\mathfrak{g}_{\delta}(0), \mathfrak{g}_{\delta}(0)\right] \subset$ $\mathfrak{g}_{\delta}(0)$ и потому $\mathfrak{g}_{\delta}(0)$ является алгеброй Ли над $\mathbb{Z}_{p}$. Исходя из формулы Кемпбелла-Хаусдорфа, можно гарантировать (уменьшив, если надо, $\delta>0$ ), что $\mathfrak{g}_{\delta}(0)$ диффеоморфно отображается посредством экспоненциального отображения ехр на некоторую компактную открытую подгруппу $G_{\delta}(1) \subset G$. Ясно, что, взяв $v \in \mathfrak{g}_{\delta}(0), v \neq 0$, мы получим изоморфизм $\mathbb{Z}_{p}$ на $\exp \left(\mathbb{Z}_{p} v\right)$. В частности, $G_{\delta}(1)$ не содержит конечных (и, следовательно, дискретных) подгрупп.

Итак, в группе Ли над $\mathbb{Q}_{p}$ имеются сколь угодно малые открытые подгруппы. Следовательно, малая окрестность единицы не порождает всю группу $G$. Кроме того, экспоненщиальное отображение определено не на всей алгебре $\mathfrak{g}$, а лишь на окрестности $0 \in \mathfrak{g}$. Это делает изучение групा Ли над $\mathbb{Q}_{p}$ и их однородных пространств более сложньм. С другой стороны, $p$-адические группы Ли в некоторых отношениях устроены намного проще, чем вешественные.

4. Однопараметрической подгруппой в $G$ назьвается непрерывный гомоморфизм $\varphi: \mathbb{Q}_{p} \rightarrow G$. Заметим, что любая унипотентная одномерная подгрупа в $G L\left(n, \mathbb{Q}_{p}\right)$ является однопараметрической, так как для нильпотентной матрицы $X$ ряд

$$
\exp (t X)=\sum_{i=0}^{\infty} \frac{t^{i} X^{i}}{i !}
$$

обрывается и потому подгруппа $\exp (t X)$ определена при всех $t \in \mathbb{Q}_{p}$.

Сформулируем некоторые результаты из [R93,95a], которые весьма полезны при изучении групп Ли над $\mathbb{Q}_{p}$. Во-первых, заметим, что для любой замкнутой подруппы $H \subset G$ имеем $H \cap G_{\delta}(1) \subset \exp \left(\mathfrak{h}_{\delta}(0)\right)$, где $\mathfrak{h}$ - подалгебра Ли для $H$ (это так, поскольку из $\exp (v) \in H \cap G_{\delta}(1)$ следует $\exp \left(\mathbb{Z}_{p} v\right) \subset H$ и поэтому $\left.v \in \mathfrak{h}_{\delta}(0)\right)$. Отсюда вытекает, что предел возрастающей последовательности замкнутых подгрупш в $G$ сам является замкнутой подгруппй в $G$. Следовательно, любая однопараметрическая подгруппа замкнута (так как $\mathbb{Q}_{p}=\bigcup_{i=0}^{\infty} p^{-i} \mathbb{Z}_{p}$ и каждая подгруппа $p^{-i} \mathbb{Z}_{p}$ компактна). Поскольку в $\mathbb{Q}_{p}$ нет дискретных подгрупп, то однопараметрическая подгрупа либо тривиальна, либо замкнута и некомпактна. Более того, каждая такая подгруппа является Ad-yнипотентной в $G$. Это вытекает из теоремы [R95a] (доказанной независимо Любоцким и Прасадом) о том, что любая однопараметрическая подгруппа в $G L\left(n, \mathbb{Q}_{p}\right)$ имеет вид $\varphi(t)=\exp (t X), t \in \mathbb{Q}_{p}$, для некоторой нильпотентной матрищы $X \in g l\left(n, \mathbb{Q}_{p}\right)$. (Идея доказательства теоремы состоит в том, что в некотором конечномерном алгебраическом расширении $F$ поля $\mathbb{Q}_{p}$ подг руппа $\varphi\left(\mathbb{Q}_{p}\right)$ приводится к верхнетреугольному виду (жорданова форма!) и потому каждый ее диагональньй элемент задает непрерьвньй гомоморфизм $\lambda: \mathbb{Q}_{p} \rightarrow F^{*}=F-\{0\}$. Так как $|\lambda(s)|^{p^{n}}=\left|\lambda\left(p^{n} s\right)\right| \rightarrow 1, n \rightarrow+\infty$, то $|\lambda(s)|=1$, и поэтому образ $\lambda\left(\mathbb{Q}_{p}\right)$ компактен, а следовательно, и тривиален. Это означает, что $\varphi\left(\mathbb{Q}_{p}\right)$ состоит из строго верхнетреугольных матриц и, как следствие, имеет искомый вид.)

$\Gamma$ рупу Ли $G$ над $\mathbb{Q}_{p}$ назовем регулярной, если $Z(G)=\operatorname{Ker} \operatorname{Ad}_{G}$ и порядки всех конечных подг рупп в $G$ ограничены некоторой константой. Ясно, что группа $G L\left(n, \mathbb{Q}_{p}\right)$ 
регулярна (как и ее связные по Зарисскому алгебраические подгрупшы). Далее мы ограничимся рассмотрением регулярных групп Ли и их подгрупп Ли (в них всегда можно найти регулярную открытую подгруппу, содержашую все однопараметрические подгрупшы [R95a]). Преимушество рассмотрения именно таких групп состоит, в частности, в том, что две однопараметрические подгрупш, совпадаюшие на окрестности единицы в $G$, обязаны совпадать всюду [R95a]. Заметим, что дискретная группа $G=\mathbb{Q}_{p} / \mathbb{Z}_{p}$ нерегулярна и, положив $\varphi_{1}(x)=\mathbb{Z}_{p} \in G$ и $\varphi_{2}(x)=\left(x+\mathbb{Z}_{p}\right) / \mathbb{Z}_{p}$, мы получим две различные аддитивные подгруппы в $G$, совпадаюшие на окрестности единишы $\mathbb{Z}_{p} \in G$.

По аналогии с вешественньм случаем можно определить орищикличность однопараметрической подгрупшы $U$, связав с ней одномерную подгруппу $A \subset G$ (назьваемую диагональной для $U)$ и соответствуюшую полупростую подгрупу $S L_{2}(U, A) \subset G$ (точное определение см. в [R95a]). Регулярную группу Ли $G$ назовем редуктивной, если каждая аддитивная однопараметрическая подгруппа в $G$ орициклична (такое определение обобщает обычную редуктивность алгебраических групा). Одним из основных результатов является

Теорема 13.1 ([R95a]). Любая регулярная группа Ли $G$ над $\mathbb{Q}_{p}$, имеющая решетку, является редуктивной.

Ранее этот факт был известен для алгебраических групп Ли над $\mathbb{Q}_{p}($ см. $[\mathrm{T}])$. Итак, при вполне естественных условиях на $G$ все однопараметрические подгруппы не только унипотентны, но орицикличны, что существенно упрощает доказательство гипотез Рагунатана и Дани. Заметим, что $\mathbb{Q}_{p}^{*}$ не имеет однопараметрических подгрупп и потому является редуктивной. Что касается $\mathbb{Q}_{p}$, то в ней нет решеток.

Другая особенность поля $\mathbb{Q}_{p}$ состоит в том, что каждая решетка в группе Ли $G$ над $\mathbb{Q}_{p}$ является равномерной [R93]. Напомним, что если $\Gamma$ - дискретная подгруппа в групе Ли $G$, то последовательность $\left\{\Gamma g_{n}\right\}$ сушественно расходится в $\Gamma \backslash G$, если найдутся $\gamma_{n} \in \Gamma-\{1\}$ такие, что $g_{n}^{-1} \gamma_{n} g_{n} \rightarrow 1, n \rightarrow \infty$. Так как $G_{\delta}(1)$ не содержит дискретных подгруп, то каждое пересечение $g_{n}^{-1} \Gamma g_{n} \cap G_{\delta}(1)$ тривиально и поэтому на $\Gamma \backslash G$ нет существенно расходящихся последовательностей (независимо от выбора дискретной подгрупшы $\Gamma \subset G$ ). Отсюда следует, в частности, что каждая решетка $\Gamma \subset G$ является равномерной $[\mathrm{Rag}]$.

Основньм инструментом в доказательстве метрической гипотезы служит

TEOPEмa 13.2 ([R95a]). Пусть подгруппа $A \subset G$ диагональна для орициклической подгруппы $U$ в регулярной группе Ли $G$ над $\mathbb{Q}_{p}, \Gamma \subset G$ - дискретная подгруппа и $\mu$-вероятностная $U$-әргодическая мера на $\Gamma \backslash G$. Тогда найдется әлемент $c \in Z_{G}(U)$ такой, что $\mu$ является $c S L_{2}(U, A) c^{-1}$-инвариантной алгебраической мерой.

Если сравнить эту теорему с теоремой 7.4 , легко видеть, что в $p$-адическом случае мера $\mu$ не может быть сосредоточена на сушественно дивергентном для $A$ подмножестве в $\Gamma \backslash G$ (так как на $\Gamma \backslash G$ вообще нет сушественно дивергентных точек для действия какой-бы то ни было подгруппы в $G$ ).

В заключение сформулируем основные результаты для унипотентных потоков на однородных пространствах общего вида. Пусть $S$ - некоторое конечное множество 
различных простых чисел $p_{1}, \ldots, p_{n}$, включая, быть может, $\infty$, а $G_{i}$ - регулярная группа Ли над полем $\mathbb{Q}_{p_{i}}, i=1, \ldots, n$ (либо связная над $\mathbb{R}$, если $p_{i}=\infty$ ). Предположим, что $H$ - замкнутая подгрупа в $\prod_{i=1}^{n} G_{i}, \Gamma$ - дискретная подгрупша в $H$, и $U \subset H$ - подгруппа вида $U=\prod_{i=1}^{n} U_{i}$, где каждая $U_{i}$ порождена однопараметрическими $\mathrm{Ad}$-унипотентная подгруппами в $G_{i}, i=1, \ldots, n$ (напомним, что для $p_{i}<\infty$ условие Ad-унипотентности вьполняется автоматически). Тогда имеют место

ТеОРема 13.3 ([R95a]). Каждая U-инвариантная эргодическая вероятностная мера на $\Gamma \backslash H$ является алгебраической.

Заметим, что в [MТ94] эта теорема доказана для алгебраических груп, а в [MT96] распространена на класс почти линейных груп (заметим, что любая связная група Ли над $\mathbb{R}$ является почти линейной).

TEOPEма 13.4 ([R95a]). Если Г - решетка в H, то каждое замыкание $\overline{x U}$, $x \in \Gamma \backslash H$, является однородным пространством конечного облема.

Если подгруппа $U$ однопараметрична, т.е. $U=\left\{u(t), t \in \mathbb{Q}_{s}\right\} \subset G_{i}$ для некоторого $s=p_{i}$, то имеет место следующий результат о сходимости мер, обобщающий теорему 9.1:

Teopema 13.5 ([R95a]). Пусть $\Gamma-$ решетка в $H$ u $x_{k} \rightarrow x \in \Gamma \backslash H$. Предnoложим, что в $H$ нет замкнутой неоткрытой подгруппь $L \subset H$ такой, что $U \subset L$ и $x L$ есть пространство конечного обгема. Тогда найдется алгебраическая мера $\nu$ на $\Gamma \backslash H$ такая, что ее стабилизатор $\Lambda(\nu)$ открыт в $H, \nu(x \Lambda(\nu))=1$, действие $U$ на $(x \Lambda(\nu), \nu)$ әргодично и

$$
\lim _{k \rightarrow \infty} \frac{1}{\lambda_{s}\left(F_{s}\left(\tau_{k}\right)\right)} \int_{F_{s}\left(\tau_{k}\right)} f\left(x_{k} u(t)\right) d \lambda_{s}(t)=\int_{\Gamma \backslash H} f d \nu
$$

для любой функиии $f \in C_{c}(\Gamma \backslash G)$ и любой последовательности $\tau_{k} \rightarrow \infty, k \rightarrow \infty$.

Здесь $F_{s}(\tau)=\left\{t \in \mathbb{Q}_{s}:|t|_{s} \leqslant \tau\right\}$ и $\lambda_{s}$ - мера Хаара на $\mathbb{Q}_{s}$. Ясно, что при $x_{k} \equiv x$ мы получаем теорему о равномерной распределенности унипотентных орбит.

\section{$\S$ 14. Применения в теории чисел}

1. Как уже было сказано во введении, самьм мошным стимулом для доказательства гипотез Рагунатана стала гипотеза Оппенгейма-Давенпорта в теории чисел (см. также [Ma91b,96], [R94,95b], [B]). Назовем квадратичную форму рациональной, если она пропоршиональна форме с рациональными коэффициентами, и иррациональной в противном случае. Пусть $B\left(x_{1}, \ldots, x_{n}\right)$ - неопределенная невырожденная иррациональная квадратичная форма от $n \geqslant 3$ переменных. Гипотеза состояла в том, что для каждого $\varepsilon>0$ найдется целочисленный ненулевой вектор $x \in \mathbb{Z}^{n}-\{0\}$, для которого $|B(x)|<\varepsilon$. Методами аналитической теории чисел в 50-х годах удалось доказать данную гипотезу для $n \geqslant 21$ (история вопроса хорошо изложена в [Mа96]).

Докажем по индукции, что достаточно рассмотреть случай $n=3$ (следуя [DM89]). В самом деле, для $n>3$ рассмотрим такой рациональный базис $v_{1}, \ldots, v_{n} \in \mathbb{Q}^{n}$ в $\mathbb{R}^{n}$, что ограничение $B$ на линейную оболочку $L=\operatorname{Span}\left(v_{1}, \ldots, v_{n-1}\right)$ является 
неопределенной невырожденной формой и $B$ нетривиальна на $\operatorname{Span}\left(v_{1}, v_{2}\right)$ (это легко сделать, если привести $B$ к главньм осям, а затем пошевелить базис, сделав его рациональньм). Допустим, что эта форма на $L$ рациональна. Пусть $L_{t}=\operatorname{Span}\left(v_{1}, \ldots, v_{n-2}, v_{n-1}+t v_{n}\right)$. Тогда для малых $|t|<\delta$ ограничение $B$ на $L_{t}$ также является неопределенной невырожденной формой. Предположим, что для рационального $0<t<\delta$ форма $\left.B\right|_{L_{t}}$ рациональна. Так как ограничение $B$ на $L \cap L_{t}=\operatorname{Span}\left(v_{1}, \ldots, v_{n-2}\right)$ при $n>3$ нетривиально, то найдется $\alpha>0$ такое, что $\alpha B$ на $L$ и $L_{t}$ имеет рациональные коэффициенты. Но тогда $\alpha B$ имеет рациональные коэффициенты на всем $\mathbb{R}^{n}$. Это противоречие показывает, что ограничение $B$ на некоторую рациональную гиперплоскость $L_{t}, t \in \mathbb{Q}$, является невырожденной неопределенной иррациональной формой и можно снизить размерность до $n-1$.

Заметим, что для $n=2$ гипотеза неверна. Например, если $B\left(x_{1}, x_{2}\right)=x_{1}^{2}-\lambda^{2} x_{2}^{2}$, где $\lambda=1+\sqrt{2}$, то 0 является изолированной точкой множества $B\left(\mathbb{Z}^{2}\right)$. Дело в том, что $\sqrt{2}$ (а значит, и $\lambda$ ) плохо приближается рациональньми числами: как легко проверить, $\left|\sqrt{2}-\frac{n}{m}\right|>\frac{1}{4 m^{2}}$ для любых $n \in \mathbb{Z}, m \in \mathbb{N}$.

Рассмотрим случай $n=3$. Пусть $G=S L(3, \mathbb{R}), \Gamma=S L(3, \mathbb{Z})$ и $S(x)=2 x_{1} x_{3}-x_{2}^{2}$. Мы будем рассматривать правое действие $G$ на $\mathbb{R}^{n}$, т.е. элементы $\mathbb{R}^{n}$ будем записывать строками. Ясно, что любая невырожденная неопределенная квадратичная форма $B$ на $\mathbb{R}^{3}$ эквивалентна над $\mathbb{R}$ одной из форм $\pm S$. Поэтому если $H_{B}$ - связная компонента стабилизатора $S O(B)=\left\{g \in G: B(x g)=B(x), x \in \mathbb{R}^{n}\right\}$ формы $B$, то $H_{B}$ сопряжена связной компоненте $H$ стабилизатора формы $S$. В группе $H$ можно выделить три однопараметрические подгруппы:

$$
\left\{V_{t}=\left(\begin{array}{ccc}
1 & 0 & 0 \\
t & 1 & 0 \\
t^{2} / 2 & t & 1
\end{array}\right)\right\},\left\{A_{t}=\left(\begin{array}{ccc}
e^{t} & 0 & 0 \\
0 & 1 & 0 \\
0 & 0 & e^{-t}
\end{array}\right)\right\},\left\{V_{t}^{T}=\left(\begin{array}{ccc}
1 & t & t^{2} / 2 \\
0 & 1 & t \\
0 & 0 & 1
\end{array}\right)\right\} .
$$

Легко видеть, что $H \simeq S O(2,1)_{0} \simeq P S L(2, \mathbb{R})$ и $H$ порождается двумя унипотентными подгрупшами $V_{\mathbb{R}}$ и $V_{\mathbb{R}}^{T}$ (кстати, для $n=2$ стабилизатор неопределенной формы является редуктивной однопараметрической подгруппой в $S L(2, \mathbb{R})$, задающей геодезический поток, и в этом состоит решаюшее отличие случаев $n=2$ и $n=3)$. Рагунатан заметил, что гипотеза Оппенгейма-Давенпорта сводится к рассмотрению орбит действия $(\Gamma \backslash G, H) .{ }^{15}$

Лемма 14.1 (см. [Ма87]). Если каждая предкомпактная орбита вида ГgН, $g \in G$, является компактной, то верна гипотеза Оппенгейма-Давенпорта.

ДокАЗАТЕЛЬСТво. Если $B$ - любая невырожденная неопределенная форма на $\mathbb{R}^{3}$, то найдутся $g \in G$ и $\lambda \in \mathbb{R}$ такие, что $B(x)=\lambda S(x g)$ для всех $x \in \mathbb{R}^{3}$. Достаточно поэтому доказать, что множество $S\left(\mathbb{Z}^{3} g\right)$ имеет 0 своей предельной точкой при условии, что форма $B$ иррациональна. Предположим, что, напротив, 0 является изолированной точкой этого множества. Тогда орбита $\Gamma g H \subset \Gamma \backslash G$ предкомпактна. В самом деле, допустим, что $\Gamma g h_{k} \rightarrow \infty$ для некоторой последовательности $h_{k} \in H$. Тогда по критерию Малера D.6 точка $0 \in \mathbb{R}^{3}$ является предельной для системы решеток $\mathbb{Z}^{3} g h_{k}, k \in \mathbb{N}$. Но $S\left(x g h_{k}\right)=S(x g)$ и мы получили противоречие.

\footnotetext{
${ }^{15}$ Как замечено в [Ma96], по существу эта редукция содержалась в неявном виде еще в [CS].
} 
Итак, орбита $\Gamma g H \subset \Gamma \backslash G$ предкомпактна. По условию леммы в таком случае она даже компактна, откуда следует, что $S L(3, \mathbb{Z}) \cap g \mathrm{Hg}^{-1}$ является решеткой в $g \mathrm{Hg}^{-1}$, плотной по Зарисскому по теореме Бореля В.2. Следовательно, $g H^{-1}$ определена над $\mathbb{Q}$. Но $g \mathrm{Hg}^{-1}$ есть связная компонента стабилизатора $B$. Докажем, что тогда форма $B$ рациональна.

В самом деле, пусть $\sigma \in \operatorname{Aut}(\mathbb{C}, \mathbb{Q})$ есть любой (вообще говоря, разрьвньй) автоморфизм поля $\mathbb{C}$, неподвижньй на $\mathbb{Q}$. Он индуцирует автоморфизм $\mathbb{C}^{3}$, которьй мы будем обозначать так же, и автоморфизм $g \rightarrow g^{\sigma}$ группы $G L(3, \mathbb{C})$ применением $\sigma$ к каждому элементу матрицы $g$. Так как стабилизатор $H_{B}=g H^{-1}$ формы $B$ определен над $\mathbb{Q}$, то $H_{B}$ инвариантна относительно этого автоморфизма, и поэтому стабилизаторы $B$ и $B^{\sigma}$ совпадают. Докажем, что тогда $B=\alpha B^{\sigma}$ для некоторого $\alpha \in \mathbb{R}$. Для этого достаточно проверить, что $C=\alpha S$ для любой квадратичной формы $C$ со стабилизатором $H$. Это достигается прямой проверкой: любая симметрическая матрица $C$, удовлетворяющая двум условиям $V_{1} C V_{1}^{T}=C$ и $V_{1}^{T} C V_{1}=C$, пропорциональна матрице $S$.

Таким образом, $B^{\sigma}$ пропорциональна $B$ для любого $\sigma \in \operatorname{Aut}(\mathbb{C}, \mathbb{Q})$. Допустим, что $\alpha=B_{i j} / B_{k l} \notin \mathbb{Q}$ для некоторой пары элементов матрицы $B$. Хорошо известно, что множество неподвижных точек групш $\operatorname{Aut}(\mathbb{C}, \mathbb{Q})$ есть в точности $\mathbb{Q}$. Поэтому найдется $\sigma \in \operatorname{Aut}(\mathbb{C}, \mathbb{Q})$ такой, что $B_{i j}^{\sigma} / B_{k l}^{\sigma}=\sigma(\alpha) \neq \alpha$ и, стало быть, $B_{i j} / B_{i j}^{\sigma} \neq$ $B_{k l} / B_{k l}^{\sigma}$. Противоречие.

Итак, гипотеза Опенгейма-Давенпорта сводится к доказательству компактности предкомпактных орбит потока $(\Gamma \backslash G, H)$. По существу для этого достаточно рассмотреть предкомпактные орбиты унипотентного потока $\left(\Gamma \backslash G, V_{\mathbb{R}}\right)$ (данное наблюдение Рагунатана и повысило интерес к изучению унипотентных потоков). Эта задача с использованием структуры групш $S L(3, \mathbb{R})$ была полностью решена Маргулисом [Ma87]; мы приводим доказательство в конще параграфа. В [Mа89] был доказано и усиление гипотезы Оппенгейма-Давенпорта: для каждого $\varepsilon>0$ найдется $x \in \mathbb{Z}^{n}$, для которого $0<|B(x)|<\varepsilon$. Известно [Op], что если 0 является правой предельной точкой для $B\left(\mathbb{Z}^{n}\right)$, то он является и левой предельной точкой. Кроме того, множество $B\left(\mathbb{Z}^{n}\right)$ инвариантно относительно умножения на квадрат любого целого числа. Поэтому множество $B\left(\mathbb{Z}^{n}\right)$ плотно в $\mathbb{R}$ для любой формы $B$ указанного вида (семейство интервалов вида $\left(\frac{a-\varepsilon}{n^{2}}, \frac{a+\varepsilon}{n^{2}}\right), n \in \mathbb{N}$, содержит интервал вида $(0, \delta)$ и потому множество $B\left(\mathbb{Z}^{n}\right)$ попадает в любую $\varepsilon$-окрестность произвольной точки $\left.a>0\right)$. Итак, имеет место

ТЕОРема 14.2 ([Ma89]). Пусть В(x) - неопределенная невырожсденная ирраииональная квадратичная форма на $\mathbb{R}^{n}$, где $n \geqslant 3$. Тогда множество $B\left(\mathbb{Z}^{n}\right)$ всюду плотно в $\mathbb{R}$.

Заметим, что основная сложность в изучении потока $\left(\Gamma \backslash G, V_{\mathbb{R}}\right)$ состояла в том, что подгруппа $V_{\mathbb{R}} \subset G$ не является орисферической. Таким образом, Маргулису впервые удалось изучить устройство орбит унипотентного потока, не являющегося орисферическим (в случае полупростой группы $G$ ).

Детальное изучение всех (а не только предкомпактных) орбит действия $(\Gamma \backslash G, H)$ позволило Дани и Маргулису [DM89,90a] значительно усилить результат о значениях 
формы $B$ в целых точках. Оказалось, что любая незамкнутая орбита потока $(\Gamma \backslash G, H)$ всюду плотна в $\Gamma \backslash G$ (с точки зрения общего результата Ратнер это и не удивительно, так как между $H$ и $S L(3, \mathbb{R})$ нет других связных замкнутых подгрупп).

Скажем, что целочисленный вектор $x \in \mathbb{Z}^{n}$ является примитивным, если нет вектора $y \in \mathbb{Z}^{n}$ и целого числа $k \geqslant 2$ таких, что $x=k y$. Обозначим через $P\left(\mathbb{Z}^{n}\right)$ множество всех примитивных векторов. Заметим, что множество $P\left(\mathbb{Z}^{n}\right)$ инвариантно относительно действия $S L(n, \mathbb{Z})$. Имеет место

Теорема 14.3 ([DM89]). Пусть B $(x)$ - неопределенная невырожденная иррачиональная квадратичная форма на $\mathbb{R}^{n}$, где $n \geqslant 3$. Тогда множество $B\left(P\left(\mathbb{Z}^{n}\right)\right)$ всюду плотно в $\mathbb{R}$.

Приведем вывод этого результата из утверждения о структуре орбит. Как и при доказательстве теоремы 14.2 , заметим, что достаточно рассмотреть случай $n=3$ (единственное дополнение при этом состоит в том, что $P\left(L \cap \mathbb{Z}^{n}\right) \subset P\left(\mathbb{Z}^{n}\right)$ для любой рациональной гиперплоскости $\left.L \subset \mathbb{R}^{n}\right)$. Нам нужно доказать, что $\overline{B(E)}=\mathbb{R}$, где $E=$ $P\left(\mathbb{Z}^{3}\right)$ и $B$ - неопределенная невырожденная иррациональная квадратичная форма. Но $B(E)=B\left(E H_{B}\right)=B\left(E \Gamma H_{B}\right)$, где $\Gamma=S L(3, \mathbb{Z})$ и $H_{B}$ - стабилизатор формы $B$. Мы знаем, что орбита $\Gamma H_{B}$ либо замкнута, либо всюду плотна в $\Gamma \backslash G$. В первом случае группа $\Gamma \cap H_{B}$ по теореме D. 10 является решеткой в $H_{B}$, и поэтому $H_{B} \subset G=$ $S L(3, \mathbb{R})$ определена над $\mathbb{Q}$. Доказательство леммы 14.1 говорит о том, что форма $B$ рациональна. Следовательно, $\overline{\Gamma H_{B}}=G$ и поэтому $\overline{B(E)}=\overline{B(E G)}=\overline{B\left(\mathbb{R}^{3}\right)}=\mathbb{R}$.

2. Для квадратичной формы $P$ на $\mathbb{R}^{n}$ определим $V_{(a, b)}^{P}(T)=\{|x|<T$ : $a<P(x)<b\}$. Приведем результаты [DM93] и [EMM] об асимптотике числа $N_{(a, b)}^{P}(T)$ целых точек в $V_{(a, b)}^{P}(T)$. Пусть $n \geqslant 3,1 \leqslant p \leqslant n / 2$ и $Q(p, n)$ - пространство симметрических матрищ размера $n \times n$ с определителем \pm 1 и сигнатурой $(p, n-p)$. Каждая матрица $P \in Q(p, n)$ задает квадратичную форму $P(x)=x P x^{T}$, $x \in \mathbb{R}^{n}$, и пространство $Q(p, n)$ имеет естественную локально-компактную топологию поточечной сходимости на $\mathbb{R}^{n}$.

Первое утверждение нижеизложенной теоремы дает асимптотическую нижнюю оценку для $N_{(a, b)}^{P}(T)$ равномерно по любому подкомпакту $C \subset Q(p, n)$ в произвольном компакте $K \subset Q(p, n)$ после выкидьвания конечного числа рациональных форм в $K$. Знаменитая теорема Мейера (см. [Ca78] или [Se82]) гласит, что при $n \geqslant 5$ рациональная форма $P \in Q(p, n)$ имеет $x \in \mathbb{Z}^{n}, x \neq 0$, такой, что $P(x)=0$. Поэтому естественно предположить, что при $n \geqslant 5$ можно найти асимптотическую нижнюю оценку для $N_{(-\varepsilon, \varepsilon)}^{P}(T)$ для всех без исключения форм из произвольного компакта $K \subset Q(p, n)$. Второе утверждение теоремы подтверждает это предположение, хотя оценка получается несколько более слабой.

Teорема 14.4 ([DM93]). Пусть $K-$ произвольный компакт в $Q(p, n)$. Тогда

(1) для любых $a<b$ и любого $\delta>0$ найдется конечный набор $N \subset K$ рациональных форм такой, что для любого компакта $C \subset K-N$ существует $T_{0} \geqslant 0$, для которого

$$
N_{(a, b)}^{P}(T) \geqslant(1-\delta) \operatorname{vol}\left(V_{(a, b)}^{P}(T)\right), \quad P \in C, \quad T \geqslant T_{0} ;
$$

(2) если $n \geqslant 5$, то для любого $\varepsilon>0$ существуют $c>0$ и $T_{0} \geqslant 0$ такие, что

$$
N_{(-\varepsilon, \varepsilon)}^{P}(T) \geqslant c \operatorname{vol}\left(V_{(-\varepsilon, \varepsilon)}^{P}(T)\right), \quad P \in K, \quad T \geqslant T_{0} .
$$


Для иррациональной формы $P \in Q(p, n)$ оценка (1) (но с константой $0<c<1$ вместо $1-\delta$ ) была независимо получена Дани и Мозесом, а также Ратнер (оба результата не опубликованы). Можно показать, что $\operatorname{vol}\left(V_{(a, b)}^{P}(T)\right) \sim \lambda_{P}(b-a) T^{n-2}$, $T \rightarrow \infty$.

Приведем идею доказательства теоремы 14.4. Определим форму $S \in Q(p, n)$ на $\mathbb{R}^{n}$ с базисом $e_{1}, \ldots, e_{n}$ формулой

$$
S\left(x_{1}, \ldots, x_{n}\right)=2 x_{1} x_{n}+\sum_{2}^{p} x_{i}^{2}-\sum_{p+1}^{n-1} x_{i}^{2} .
$$

Пусть $G=S L(n, \mathbb{R}), \Gamma=S L(n, \mathbb{Z}), H=S O(S)$. Так как любая форма $B \in Q(p, n)$ заменой переменных может быть преврашена в $S$, то $Q(p, n)$ гомеоморфно $H \backslash G$. Пусть $U \subset H$ фиксирует $e_{1}, \ldots, e_{n-2}$ и оставляет инвариантными подпространства, натянутые на $e_{1}, e_{n-1}$ и $e_{1}, e_{n-1}, e_{n}$. Тогда $U$ является однопараметрической унипотентной подгруппой (и в случае $(p, n)=(1,3)$ она совпадает с $\left.V_{\mathbb{R}}\right)$. Через $M \simeq S O(p-1, \mathbb{R})$ обозначим стабилизатор в $H$ подпространства $\mathbb{R}\left(e_{1}+e_{n}\right)+\mathbb{R} e_{2}+\cdots+\mathbb{R} e_{p}$, на котором форма $S$ положительно определена.

Правое действие $G$ на $\mathbb{R}^{n}$ задает гомеоморфизм $\Gamma \backslash G$ и пространства решеток в $\mathbb{R}^{n}$. Всякой измеримой функции $\psi$ на $\mathbb{R}^{n}$, обрашающейся в нуль вне некоторого компакта, можно сопоставить функцию $\tilde{\psi}$ на $\Gamma \backslash G$ по формуле

$$
\tilde{\psi}(\Gamma g)=\sum_{x \in \mathbb{Z}^{n} g} \psi(x)
$$

При этом

$$
\int_{\mathbb{R}^{n}} \psi d x=\int_{\Gamma \backslash G} \tilde{\psi} d \nu
$$

Доказательство теоремы 14.4 опирается на следующее очевидное тождество:

$$
\int_{T}^{k T} \int_{A} \sum_{v \in \mathbb{Z}^{n} g} \psi\left(v m U_{t}\right) d m d t=\int_{T}^{k T} \int_{A} \tilde{\psi}\left(\Gamma g m U_{t}\right) d m d t
$$

для всякой ограниченной измеримой функции $\psi$ на $\mathbb{R}^{n}$ с компактным носителем, любого борелевского подмножества $A \subset M, k>1$ и $T>0$. Идея состоит в том, что для подходяшего выбора $\psi$ и $g \in G$ левая часть этого тождества (при $A=M$ ) дает хорошее приближение $N_{(a, b)}^{P}(T)$, а асимптотика правой части оценивается с помошью теоремы 8.1 о равномерной распределенности. Однако нужно иметь ввиду, что функция $\tilde{\psi}$ не является ограниченной, если $\psi \neq 0$, и поэтому теорему 8.1 (точнее, ее равномерньй аналог 9.1b) приходится применять к некоторой ограниченной непрерьвной функции $f \leqslant \tilde{\psi}$.

Ясно, что этот метод не позволяет получить асимптотическую оценку сверху для $N_{(a, b)}^{P}(T)$. Это затруднение было недавно преодолено Эскиным, Маргулисом и Мозесом [EMM]. Оказьвается, оценка сверху правой части $(*)$ все-таки получается 
применением теоремы о равномерной распределенности, если доказать, что для некоторого $\delta>0$

$$
\lim \sup _{T \rightarrow \infty} \frac{1}{T} \int_{0}^{T} \int_{A} \tilde{\psi}\left(\Gamma g m U_{t}\right)^{1+\delta} d m d t<\infty .
$$

В [EMM], по существу, доказывается это неравенство для любого $0 \leqslant \delta<1$ в случае, если $(p, n) \neq(1,3)$ и $(2,4)$. Для исключительных значений $(p, n)$ и $\delta=0$ неравенство $(* *)$ становится верным, если заменить $1 / T$ на $1 /(T \log T)$. В итоге доказывается следуюший важньй результат.

TeOPEMa 14.5 ([EMM]). Пусть $K$ - произвольный компакт в $Q(p, n)$ и а $<$. Тогда

(1) если $(p, n) \neq(1,3) u(p, n) \neq(2,4)$, то найдется константа $c=c(K, a, b)$ такая, что $N_{(a, b)}^{P}(T)<c T^{n-2}$ для всех $P \in K, T>1$. Более того, для любого $\delta>0$ найдется конечный набор $N \subset K$ рачиональных форм такой, что для любого компакта $C \subset K-N$ существует $T_{0} \geqslant 0$, для которого

$(1-\delta) \lambda_{P}(b-a) T^{n-2} \leqslant N_{(a, b)}^{P}(T) \leqslant(1+\delta) \lambda_{P}(b-a) T^{n-2}, \quad P \in C, \quad T \geqslant T_{0}$

(2) если $(p, n)=(1,3)$ или $(p, n)=(2,4)$, то найдется константа $c=c(K, a, b)$ такая, что

$$
N_{(a, b)}^{P}(T)<c T^{n-2} \log T, \quad P \in K, \quad T>2 .
$$

При этом для исключительных значений $(p, n)$ асимптотика $N_{(a, b)}^{P}(T) \sim T^{n-2}$ выполняется для почти всех форм $P \in Q(p, n)$ и нарушается для форм вида $x_{1}^{2}+x_{2}^{2}-\alpha x_{3}^{2}$ и $x_{1}^{2}+x_{2}^{2}-\alpha\left(x_{3}^{2}+x_{4}^{2}\right)$, если $\alpha$ слишком быстро приближается квадратами рациональных чисел $[\mathrm{EMM}]$. Первые примеры квадратичных форм $P$, для которых нарушается асимптотика $N_{(a, b)}^{P}(T) \sim T^{n-2}$, были обнаружены Сарнаком.

3. Перейдем к рассмотрению гипотезы Оппенгейма-Давенпорта в так называемом $S$-арифметическом контексте, следуя Борелю и Прасаду $[\mathrm{BP}]$; см. также [R95b], [Ma96]. Пусть $S$ - конечный набор простых чисел, включая $\infty$. Тогда каждое число $s \in S$ задает $s$-адическую метрику $|\cdot|_{s}$ на поле $k=\mathbb{Q}$, и пополнение по этой метрике приводит к полю $\mathbb{Q}_{s} s$-адических чисел (будем считать, что $\mathbb{Q} \infty=\mathbb{R}$ ). Рассмотрим аддитивную локально-компактную (по отношению к топологии произведения) группу $k_{S}=\sum_{s \in S} k_{s}$. Обозначим через $O_{S} \subset k_{S}$ кольцо $S$-целых чисел. По определению оно состоит из диагональных элементов вида $(x, \ldots, x) \in \mathbb{Q}^{n}$ таких, что $|x|_{p} \leqslant 1$ для всех $p \notin S$ (т.е. $x=m / n$, где $m \in \mathbb{Z}$ и $n \in \mathbb{N}$ раскладьвается в произведение степеней простых чисел из набора $S$ ). Легко проверить, что $O_{S}$ является дискретной подгруппой в $k_{S}$ и, более того, $O_{S}$ является в $k_{S}$ равномерной решеткой (примечание в $\S 12$ дает $O_{S}$ для $S=\{p, \infty\}$ ).

По отношению к операции (покоординатного) умножения $k_{S}$ является кольцом и мы вправе рассматривать квадратичные формы на $k_{S}^{n}$. Ясно, что любую квадратичную форму $F$ на $k_{S}^{n}$ можно трактовать как набор квадратичных форм $F_{s}$ на $\mathbb{Q}_{s}^{n}$. Форма $F$ 
называется невырожденной, если таковы все $F_{s}, s \in S$. Чтобы сформулировать гипотезу Оппенгейма-Давенпорта, нужно еше правильно определить понятия неопределенности и рациональности. Скажем, что невырожденная форма $F$ является изотропной, если каждая форма $F_{s}$ изотропна, т.е. найдется ненулевой элемент $x_{s} \in \mathbb{Q}_{s}^{n}-\{0\}$ такой, что $F_{s}\left(x_{s}\right)=0$. Для $s=\infty$ это равносильно неопределенности и изотропность можно понимать как обобщение неопределенности на $S$-арифметический случай.

Форму $F$ назовем рациональной, если найдутся форма $F_{0}$ с коэффициентами в $\mathbb{Q}^{n}$ и обратимьй элемент $\lambda \in k_{S}$ такие, что $F=\lambda F_{0}$; в противном случае $F$ назьвается иррациональной. Заметим, что для рациональной формы $F$ множество $F\left(O_{S}^{n}\right)$ ее значений в $S$-целых точках является дискретным (в самом деле, форму $F_{0}$ можно выбрать с коэффициентами в $\mathbb{Z}^{n}$ и тогда $\left.F\left(O_{S}^{n}\right) \subset \lambda O_{S}\right)$. По аналогии со случаем $k_{S}=\mathbb{R}$ естественно предположить, что для иррациональной формы $F$ множество $F\left(O_{S}^{n}\right)$ недискретно в окрестности $0 \in k_{S}$ при $n \geqslant 3$.

ТЕОРема 14.6 ([BP]). Пусть $F$ - изотропная невырожденная көадратичная форма на $k_{S}^{n}$, где $n \geqslant 3$. Тогда следующие условия әквивалентны:

(1) F иррациональна,

(2) для каждого $\varepsilon>0$ найдется $x \in O_{S}^{n}$ такой, что $0<\left|F_{s}\left(x_{s}\right)\right|_{s}<\varepsilon$ для всех $s \in S$.

Борель и Прасад доказали эту теорему, следуя идеям Маргулиса из [Ма87]. В частности, они свели задачу к случаю $n=3$ и рассмотрели структуру орбит однородных действий на $S L\left(3, O_{S}\right) \backslash S L\left(3, k_{S}\right)$. Интересно, что в $S$-арифметическом случае не удается так легко вьвести плотность $F\left(O_{S}^{n}\right)$ в $k_{S}$, как для $k_{S}=\mathbb{R}$. Борель и Прасад, однако, заметили, что плотность $F\left(O_{S}^{n}\right)$ в $k_{S}$ можно установить, если доказать топологическую гипотезу Рагунатана в $S$-арифметическом контексте, что и было проделано в [R95a]. Таким образом, имеет место

ТЕОРЕМА 14.7 ([B]). В условиях теоремы 14.6 условия (1) и (2) эквивалентны условию

(3) множество значений $F\left(O_{S}^{n}\right)$ плотно в $k_{S}$.

Теорема 14.3 о плотности значений $F$ в примитивных точках $P\left(O_{S}^{n}\right)$ может быть усилена (даже для случая $k_{S}=\mathbb{R}$ ) следуюшим образом. Скажем, что набор векторов $x_{1}, \ldots, x_{m} \in O_{S}^{n}, m \leqslant n$, является примитивным, если его можно дополнить до базиса в $O_{S}^{n}$ над $O_{S}$. При $m<n$ это равносильно тому, что найдется целочисленньй элемент $g \in S L\left(n, O_{S}\right)$ такой, что $g\left(x_{i}\right)=e_{i}, i=1, \ldots, m$, где $e_{i}-i$-й элемент стандартного базиса в $O_{S}^{n}$. Ясно, что для $m=1$ это определение равносильно тому, что $x \in P\left(O_{S}^{n}\right)$, и всякий такой элемент содержится в некотором примитивном $(n-1)$-наборе. Имеет место

ТЕОРема 14.8 ([B]). Пусть $F$ - изотропная невьрохсденая иррачиональная квадратичная форма на $k_{S}^{n}$, где $n \geqslant 3$, и заданьи произвольные числа $\lambda_{1}, \ldots, \lambda_{n-1}$ $\in k_{S}$. Тогда найдется такая последовательность примитивных $(n-1)$-наборов $x_{1}^{j}, \ldots, x_{n-1}^{j} \in O_{S}^{n}, j=1, \ldots, \infty$, что $F\left(x_{i}^{j}\right) \rightarrow \lambda_{i}, j \rightarrow \infty$, для всех $i=1, \ldots, n-1$. В частности, множество $B\left(P\left(O_{S}^{n}\right)\right)$ плотно в $k_{S}$. 
ЗАмЕчАниЕ. На самом деле в $[\mathrm{BP}],[\mathrm{B}]$ рассмотрен несколько более обший случай, чем тот, что мы описали: в качестве исходного поля $k$ можно взять любое числовое поле, т.е. конечное расширение $\mathbb{Q}$.

Наконец, заметим, что асимптотическая оценка 14.4 верна и в $S$-арифметическом контексте [МТ94].

4. Теперь рассмотрим вопрос об асимптотике числа целых точек на алгебраических многообразиях в $\mathbb{R}^{n}$. Пусть решетка $\mathbb{Z}^{n} \subset \mathbb{R}^{n}$ задает соответствуюшую $\mathbb{Q}$-структуру. Предположим, что $V \subset \mathbb{R}^{n}$ - алгебраическое многообразие над $\mathbb{Q}$ (т.е. множество нулей некоторого полинома на $\mathbb{R}^{n}$ с рациональными коэффициентами) и через $V_{\mathbb{Z}}=V \cap \mathbb{Z}^{n}$ обозначим множество целых точек в $V$. Нас будет интересовать асимптотика числа точек в пересечении $V_{\mathbb{Z}} \cap D_{T}$ множества $V_{\mathbb{Z}}$ с евклидовым шаром $D_{T}$ радиуса $T$ при $T \rightarrow \infty$.

Мы дополнительно предположим, что $V$ является орбитой действия некоторой редуктивной алгебраической $\mathbb{Q}$-групш $G \subset S L(n, \mathbb{R})$, т.е. $V=v_{0} G$ для некоторого элемента $v_{0} \in V_{\mathbb{Z}}$. Тогда стабилизатор $H=\left\{g \in G: v_{0} g=v_{0}\right\}$ также является редуктивной алгебраической $\mathbb{Q}$-групшой и $V \simeq H \backslash G$. Предположим дополнительно, что $G$ и $H$ не допускают нетривиальных $\mathbb{Q}$-характеров. Тогда по теореме Бореля и Хариш-Чандры $G_{\mathbb{Z}}$ и $H_{\mathbb{Z}}$ являются решетками и $V_{\mathbb{Z}}$ есть конечное объединение $G_{\mathbb{Z}}$-орбит. Таким образом, достаточно оценить число $N(T, V, \mathscr{O})$ целых точек с нормой не более $T$ в орбите $\mathscr{O}=v_{0} G_{\mathbb{Z}}$.

Через $\lambda$ обозначим $G$-инвариантную меру на $H \backslash G$, индуцированную мерами Хаара на $G$ и $H$. Пусть $R_{T}=\left\{H g: v_{0} g \in D_{T}\right\} \subset H \backslash G$ есть образ $V \cap D_{T}$ при отождествлении $V \simeq H \backslash G$. Имеет место

Tеорема 14.9 ([DRS], [EM]). Если $V \subset \mathbb{R}^{n}$ является аффинным симметрическим многообразием и решетка $G_{\mathbb{Z}}$ неприводима в $G$, то асимптотически $N(T, V, \mathscr{O}) \sim \lambda\left(R_{T}\right)$.

Условие на $V$ здесь эквивалентно тому, что стабилизатор $H$ является неподвижной подгруппой некоторой инволюции на $G$. Основная идея состоит в доказательстве предельного перехода $\mu_{H} g \rightarrow \mu_{G}$ при $H g \rightarrow \infty$ в $H \backslash G$, где $\mu_{H}$ и $\mu_{G}$ обозначают вероятностные инвариантные меры на $H_{\mathbb{Z}} \backslash H$ и $G_{\mathbb{Z}} \backslash G$ соответственно (на самом деле достаточно доказать это соотношение "в среднем" по $R_{T}$ при $T \rightarrow \infty$ ). Эскин, Мозес и Шах в [EMS96] нашли способ избавиться от условия симметричности $V$. Напомним, что согласно доказанной ими теореме 9.8 все предельные вероятностные меры для семейства $\left\{\mu_{H} g, g \in G\right\}$ являются алгебраическими. Более того, если $H_{0}$ является максимальной связной алгебраической $\mathbb{Q}$-подгруппой в $G$ (это так в условиях теоремы 14.9), то согласно следствию 9.9 предельньй переход также имеет место. Следовательно, выполняется такая же асимптотическая оценка, как в теореме 14.9 .

В обшем случае имеются двапрепятствия. Во-первых, семейство мер $\left\{\mu_{H} g, g \in G\right\}$ должно быть относительно компактным (иначе можно получить сходимость к мере, сосредоточенной на бесконечности) и по теореме 9.7 критерием для этого служит компактность пространства $\pi\left(Z_{G}(H)\right) \subset G_{\mathbb{Z}} \backslash G$, где $\pi: G \rightarrow G_{\mathbb{Z}} \backslash G$ - естественная проекция (это эквивалентно тому, что $H$ не содержится в параболической $\mathbb{Q}$-подгруппе в $G)$. Во-вторых, множества $R_{T} \subset H \backslash G$ при $T \rightarrow \infty$ должны "равномерно охватьвать" все пространство $H \backslash G$. Следуя [EMS96], скажем, что последовательность 
$R_{T_{n}} \subset H \backslash G$ фокусируется к $H \backslash L$, где $L \supset H_{0}$ - некоторая редуктивная $\mathbb{Q}$-подгруппа в $G$, если найдется компакт $C \subset G$ такой, что

$$
\lim _{n \rightarrow \infty} \frac{\lambda\left(q\left(\left(Z_{G}\left(H_{0}\right) \cap G_{\mathbb{Z}}\right) L C\right) \cap R_{T_{n}}\right)}{\lambda\left(R_{T_{n}}\right)}>0,
$$

где $q: G \rightarrow H \backslash G$ - естественная проекция. Заметим, что множество $q\left(\left(Z_{G}\left(H_{0}\right) \cap\right.\right.$ $\left.G_{\mathbb{Z}}\right) L C$ ) замкнуто в $H \backslash G$. В самом деле, ввиду условия на $L$ произведение $G_{\mathbb{Z}} L$ замкнуто в $G$ и, стало быть, $\left(Z_{G}\left(H_{0}\right) \cap G_{\mathbb{Z}}\right) L$ замкнуто в $G$ и потому имеет замкнутый образ в $H \backslash G$.

Следует заметить, что наличие фокусировки, хотя и является довольно редким феноменом, не является невозможным: в [EMS96] приводится пример, когда для $G=$ $S L(2, \mathbb{C}), L \simeq S L(2, \mathbb{R})$ и диагональной подгрупшы $H \subset L$ последовательность $R_{n} \subset$ $H \backslash G$ фокусируется к $H \backslash L$ при $n \rightarrow \infty$.

Теперь мы можем сформулировать основной результат.

Teоpema 14.10 ([EMS96]). Пусть $H \subset G-$ две редуктивные $\mathbb{Q}$-подгруппь в $S L(n, \mathbb{R})$, причем $H_{\mathbb{Z}}$ и $G_{\mathbb{Z}}$ являются в них решетками и $V=v_{0} G \simeq H \backslash G$ для некоторого $v_{0} \in \mathbb{Z}^{n}$. Пусть $H$ не содержится в параболической $\mathbb{Q}$-подгруппе в $G$ и никакая последовательность $R_{T_{n}}=V \cap D_{T_{n}} \subset H \backslash G$ не является фокусированной. Тогда число чельх точек в пересечении $V_{\mathbb{Z}} \cap D_{T}$ асимптотически есть $\lambda\left(R_{T}\right)$.

Эскин, Мозес и Шах приводят конкретньй пример применения теоремы 14.10. Рассмотрим монический неприводимьй над $\mathbb{Q}$ полином $p$ степени $n$ с цельми коэффициентами и норму Гильберта-Шмидта на пространстве $M(n, \mathbb{R})$ матриц размера $n \times n$ (т.е. $\left.\left\|\left(A_{i, j}\right)\right\|=\sqrt{\sum_{i, j} A_{i, j}^{2}}\right)$. Пусть $V_{p}=\{A \in M(n, \mathbb{R}): \operatorname{det}(t E-A)=p(t)\}$ и $N\left(T, V_{p}\right)-$ число элементов в $V_{p}(\mathbb{Z})=V_{p} \cap M(n, \mathbb{Z})$ с нормой $\leqslant T$. Тогда $V_{p}$ является замкнутой $G$-орбитой некоторой матрицы $v_{0} \in V_{p}(\mathbb{Z})$ относительно действия сопряжением группы $G$ матрищ размера $n \times n$ с определителем \pm 1 . Централизатор $H$ элемента $v_{0}$ является максимальным $\mathbb{Q}$-тором без нетривиальных $\mathbb{Q}$-характеров. Стоит подчеркнуть, что $H$ не есть неподвижная подгруппа какой-либо инволюции и поэтому теорема 14.9 в данном случае неприменима. В [EMS96] с использованием теоремы 14.10 выводится оценка $N\left(T, V_{p}\right) \sim c_{p} T^{n(n-1) / 2}$, где константа $c_{p}$ может быть явно вычислена.

5. С помощью техники однородных потоков недавно был достигнут значительньй прогресс в теории диофантовых аппроксимаций. Для $x, y \in \mathbb{R}^{n}$ введем обозначения

$$
\begin{gathered}
x \cdot y=\sum_{1}^{n} x_{i} y_{i}, \quad\|x\|=\max _{1 \leqslant i \leqslant n}\left|x_{i}\right|, \\
\Pi(x)=\prod_{1}^{n}\left|x_{i}\right|, \Pi_{+}(x)=\prod_{1}^{n}\left|x_{i}\right|_{+}, \quad \text { где }|a|_{+}=\max (|a|, 1) .
\end{gathered}
$$

Скажем, что вектор $y \in \mathbb{R}^{n}$ очень хорошо аппроксимируется (VWA), если найдутся $\varepsilon>0$ и бесконечное число различных $q \in \mathbb{Z}^{n}$ таких, что $|q \cdot y+p| \cdot\|q\|^{n} \leqslant\|q\|^{-n \varepsilon}$ для некоторого $p=p(q) \in \mathbb{Z}$. Известно [Са57], что почти все $y \in \mathbb{R}^{n}$ не являются VWA. 
Более сложный вопрос возникает, если рассматривать ту же задачу для аналитического $k$-мерного подмногообразия $M \subset \mathbb{R}^{n}=\{f(x), x \in U\}$, где $U$ - открытое подмножество в $\mathbb{R}^{k}$ и $f: U \rightarrow \mathbb{R}^{n}$ - аналитическое отображение. Разумеется, нужно рассматривать изогнутые многообразия, т.е. те, для которых функции $1, f_{1}, \ldots, f_{n}$ линейно независимы над $\mathbb{R}$. Так, гипотеза Малера о том, что почти все точки на кривой $K=\left\{x, x^{2}, \ldots, x^{n}\right\}$ не являются VWA, была доказана Спринджуком в 60-х годах (см. обзор [Sp]). В обшем случае вопрос оставался открытым.

Более общо, скажем, что $y \in \mathbb{R}^{n}$ очень хорошо аппроксимируется мультипликативно (VWMA), если найдутся $\varepsilon>0$ и бесконечное число различных $q \in \mathbb{Z}^{n}$ таких, что $|q \cdot y+p| \cdot \Pi_{+}(q) \leqslant \Pi_{+}(q)^{-\varepsilon}$ для некоторого $p=p(q) \in \mathbb{Z}$. Ясно, что VWA $\Rightarrow$ VWMA. Поэтому еше сложнее доказать, что почти все точки многообразия $M \subset \mathbb{R}^{n}$ не являются VWMA. Так, аналог гипотезы Малера для кривой $K \subset \mathbb{R}^{n}$ (назьваемый гипотезой Бейкера) оставался открытым. Усилив теоремы Дани-Маргулиса D.7, D.8 о возврашаемости унипотентных траекторий, Клейнбок и Маргулис в итоге решили все эти проблемы:

ТЕОРеMA 14.11 ([КМ97]). Почти все точки изогнутого аналитического многообразия $M \subset \mathbb{R}^{n}$ не являются VWMA.

Важные результаты также были недавно получены в задаче об асимптотической оценке числа целых точек внутри многогранников. Пусть $P \subset \mathbb{R}^{n}$ - многогранник и $N\left(P, \mathbb{Z}^{n}\right)$ - число узлов решетки $\mathbb{Z}^{n}$ внутри $P$. Введем остаточный член $R\left(P, \mathbb{Z}^{n}\right)=$ $\operatorname{vol} P-N\left(P, \mathbb{Z}^{n}\right)$. Легко видеть, что $R\left(t P, \mathbb{Z}^{n}\right)=O\left(t^{n-1}\right)$, причем эта оценка достигается, например, для единичного куба $P$ с вершинами из $\mathbb{Z}^{n}$. Первые примеры многоугольников $P \subset \mathbb{R}^{2}$ с аномально мальм остатком $R\left(t P, \mathbb{Z}^{2}\right)=O(\log t)$ были ранее построены М. М. Скригановым с помошью плохо аппроксимирумых чисел (см. ссылки в $[\mathrm{Skr}])$. Для фиксированного многогранника $P \subset \mathbb{R}^{n}$ интересно найти типичную асимптотику $R(t P, \Gamma)$ в классе всех унимодулярных решеток $\Gamma=\mathbb{Z}^{n} g, g \in S L(n, \mathbb{R})$. Оказывается, $R(t P, \Gamma)=O\left((\log t)^{n-1+\varepsilon}\right)$ для почти всякой унимодулярной решетки $\Gamma \subset \mathbb{R}^{n}$ и произвольно малого $\varepsilon>0$. Этот результат был получен в [Skr] с помошью изучения динамики диагонального потока $(S L(n, \mathbb{Z}) \backslash S L(n, \mathbb{R}), H), \operatorname{dim} H=n-1$.

6. В заключение мы докажем гипотезу Оппенгейма-Давенпорта, следуя [DM90b] и [Ma90b]. Пусть $G=S L(3, \mathbb{R}), \Gamma=S L(3, \mathbb{Z})$, подгруппы $\left\{V_{t}\right\}$ и $\left\{A_{t}\right\}$ те же, что и в п. 1, и

$$
U_{t}=\left(\begin{array}{lll}
1 & 0 & 0 \\
0 & 1 & 0 \\
t & 0 & 1
\end{array}\right), \quad \sigma_{0}=\left(\begin{array}{ccc}
0 & 0 & 1 \\
0 & -1 & 0 \\
1 & 0 & 0
\end{array}\right), \quad q_{0}=\left(\begin{array}{lll}
0 & 0 & 0 \\
0 & 0 & 0 \\
0 & 0 & 1
\end{array}\right) .
$$

Определим квадратичную форму $S(x)=x \sigma_{0} x^{T}$, т.е. $S\left(x_{1}, x_{2}, x_{3}\right)=2 x_{1} x_{3}-x_{2}^{2}$ (напомним, что элементы $\mathbb{R}^{n}$ рассматриваются как строки и $G$ действует на $\mathbb{R}^{n}$ справа). Пусть $H \subset G$ - связная компонента стабилизатора формы $S$. Нашей целью является

Теорема 14.12 ([Ма87]). Если замыкание $\overline{x H} \subset \Gamma \backslash G$ компактно, то орбита xн компактна.

Введем следуюшие обозначения:

$$
A=A_{\mathbb{R}}, U=U_{\mathbb{R}}, V=V_{\mathbb{R}}, B=A \cdot(U \times V), F=A \cdot V, U^{+}=U_{\mathbb{R}^{+}}, U^{-}=U_{\mathbb{R}^{-}} .
$$

Ясно, что теорема 14.12 вытекает из следуюших двух утверждений. 
Лемма 14.13. Замыкания $\overline{x U^{+} H}$ и $\overline{x U^{-H}}$ некомпактны для всякой точки $x \in \Gamma \backslash G$.

ДоказАтельство. Множество $\left\{x \in \mathbb{R}^{3}: x_{3}>0, S(x)<0\right\}$ содержит в себе шар сколь угодно большого диаметра. Пусть $x=\Gamma g$. Тогда найдется такая точка $y \in \mathbb{Z}^{3} g$, что $y_{3}>0$ и $S(y)<0$. Но $S\left(y U_{t}\right)=S(y)+2 t y_{3}^{2}$. Поэтому $0 \in S\left(y U^{+}\right)$и, стало быть, $0 \in \overline{y U^{+} H}$. По критерию Малера D.6 замыкание $\overline{x U^{+} H}$ некомпактно.

ЛЕмма 14.14. Пусть некомпактная орбита $x H \subset \Gamma \backslash G$ имеет компактное замыкание. Тогда найдется точка $y \in \overline{x H}$ такая, что либо $y U^{+}$, либо уU содерәится в $\overline{x H}$.

Приступим к доказательству леммы 14.14. Фактически оно основано на следующем наблюдении, доказательство которого предоставляется читателю.

УТВЕРЖДЕНИЕ 14.15. Пусть группа Ли $G$ непрерывно действует справа на топологическом пространстве $\Omega$. Предположим, что замкнутые подмножества $Z, W \subset \Omega$ инвариантны относительно замкнутых подгрупп $V, H \subset G$ соответственно, причем $Z$ является компактным $V$-минимальным мнодсеством. Наконец, пусть $Z m \cap W \neq \varnothing$ для всякого $m \in M \subset G$. Тогда $Z h \subset W$ для всякого $h \in N_{G}(V) \cap \overline{V M H}$.

Докажем, что лемма 14.14 вьводится из следующих двух результатов.

ЛЕмма 14.16. Пусть $Y \subset X \subset \overline{x H} \subset \Gamma \backslash G$, причем множество $Y$ компактно и минимально относительно $F$, а множество $X$ минимально относительно $H$. Предположим, что $\overline{x H} \neq x H$ и $y \in Y$. Тогда замыкание множества $\{g \in G-$ $U H: y g \in \overline{x H}\}$ содержит единичный әлемент $1 \in G$.

Лемма 14.17. Пусть $M \subset G-U H u 1 \in \bar{M}$. Тогда $\overline{V M H}$ содержит либо $U^{+}$, либо $U^{-}$

В самом деле, компактное замькание $\overline{x H}$ содержит компактное $H$-инвариантное минимальное множество $X$. В свою очередь, $X$ содержит $F$-минимальное множество $Y$, а оно содержит $V$-минимальное множество $Z$. Пусть $z \in Z$ и $M=\{g \in G-$ $U H: z g \in \overline{x H}\}$. Тогда $1 \in \bar{M}$ (лемма 14.16) и $\overline{V M H}$ содержит либо $U^{+}$, либо $U^{-}$ (лемма 14.17). Но в силу утверждения $14.15 Z\left(N_{G}(V) \cap \overline{V M H}\right) \subset \overline{x H}$ и, поскольку $U \subset N_{G}(V)$, лемма 14.14 доказана.

Приступим к доказательству леммы 14.16. Вначале приведем элементарное

УТВЕРЖДЕНИЕ 14.18. Пусть группа Ли $G$ непрерывно и транзитивно действует справа на топологическом пространстве $\Omega$ и замкнутое подмножество $Y \subset \Omega$ инвариантно относительно замкнутой подгруппы $F \subset G$. Через $G_{y}$ обозначим стабилизатор точки $у \in Y$. Тогда

а) если замыкание множества $\left\{g \in G-G_{y} F: y g \in Y\right\}$ не содержит $1 \in G$, то орбита уF открыта в $Y$;

b) пусть подгруппа $B \subset G$ замкнута и содерәит $F, B_{y}=B \cap G_{y}$ и $\pi: B \rightarrow$ $B_{y} \backslash B$ - естественное отображение; предположим, что множество $Y$ компактно и F-минимально, а замыкание $\overline{\pi(F)}$ некомпактно; тогда замыкание подмножества $\{g \in G-B: y g \in y F\}$ содержит $1 \in G$. 
Легко проверить, что дискретная подгруппа $\Lambda \subset B=A \cdot(U \times V)$ либо лежит в нильрадикале $U \times V$, либо сопряжена подгрупе в $A$. В любом случае замыкание $\overline{\Lambda F} \subset \Lambda \backslash B$ некомпактно. Теперь из $14.18 \mathrm{~b})$ сразу следует

ПреДлОЖенИЕ 14.19. Пусть $Y$ - компактное $F$-минимальное множество и $y \in Y$. Тогда замыкание множества $\{g \in G-B: y g \in y F\}$ содержит $1 \in G$.

На векторном пространстве $E$ симметрических матрищ порядка $3 \times 3$ определим правое действие групшы $G$ правилом $\sigma \circ g=g^{-1} T \sigma\left(g^{-1}\right)^{T}, g \in G, \sigma \in E$. Следуюший результат проверяется непосредственным вычислением.

ПРЕДЛОЖЕНИЕ 14.20. а) $\sigma_{0} \circ U_{t}=\sigma_{0}-2 t q_{0}$ и поэтому $\sigma_{0} \circ\left(H U_{t}\right)=\sigma_{0}-2 t q_{0}$ для всех $t \in \mathbb{R}$,

b) если $g \in G$ u $\sigma_{0} \circ g=\sigma_{0}-t q_{0}$, mo $g \in H U_{t}$,

c) отображение $\left(U_{t}, h\right) \rightarrow U_{t} h$ задает гомеоморфизм $U \times H \rightarrow U H$.

Отсюда легко вьводится

ПреДЛОЖЕНИЕ 14.21. а) Пусть $h \in H \quad u q_{0} \circ h=t q_{0}$ для некоторого $t \in \mathbb{R}$. Тогда $h \in F$.

b) Если $h \in H, t \neq 0$ u $U_{t} h \in H U$, mo $h \in F$.

Теперь мы от противного докажем лемму 14.16. Пусть найдется окрестность $O(1) \subset G$ такая, что

$$
O(1) \cap\{g \in G: y g \in \overline{x H}\} \subset U H .
$$

Если орбита $y H$ открыта в $\overline{x H} \supset X$, то ввиду $H$-минимальности $X=\overline{y H}$ орбита $y H$ компактна и $y H=\overline{x H}$, что противоречит условию $x H \neq \overline{x H}$. Таким образом, орбита $y H$ не открыта в $\overline{x H}$. В силу 14.18а) замыкание множества $\{g \in G-H: y g \in \overline{x H}\}$ содержит $1 \in G$. Тогда ввиду $\left({ }^{*}\right)$ найдутся последовательности $h_{i} \in H, t_{i} \neq 0$ такие, что $y U_{t_{i}} h_{i} \in \overline{x H}$ и $U_{t_{i}} h_{i} \rightarrow 1$. Тогда $y U_{t_{i}} \in \overline{x H}$ и ввиду 14.20 c) $t_{i} \rightarrow 0, i \rightarrow \infty$.

Предложение 14.19 гарантирует, что замыкание множества $\{g \in G-B: y g \in y F\}$ содержит $1 \in G$. Поэтому найдутся $u \in O(1) \cap(G-B)$ и $p \in F$ такие, что $y u=y p . \quad$ Но тогда ввиду $(*) \quad u=U_{s} h$ для некоторых $h \in H-F$ и $s \in \mathbb{R}$. Так как $t_{i} \rightarrow 0$, для некоторого $k$ будет $u p^{-1} U_{t_{k}} p \in O(1)$. С другой стороны, так как $y U_{t_{k}} \in \overline{x H}$, то $y u p^{-1} U_{t_{k}} p=y p p^{-1} U_{t_{k}} p=y U_{t_{k}} p \in \overline{x H} p=\overline{x H}$. Теперь в силу $(*) \quad U_{s} h p^{-1} U_{t_{k}} p \in U H$, и поэтому $p^{-1} U_{-t_{k}} p h^{-1} \in H U$. Но $h^{-1} \in H$ и $p^{-1} U_{-t_{k}} p \in U-\{1\}$. Теперь из 14.21b) следует, что $h \in F$, и мы пришли к противоречию.

Нам осталось доказать лемму 14.17. Индукцией по размерности векторного пространства несложно доказать следуюшее свойство унипотентных линейных отображений.

ПРЕДЛОЖЕНИЕ 14.22. Пусть $E$ - векторное пространство и $E_{0} \subset E-$ подпространство неподвижных точек для унипотентной подгруппьи $V_{\mathbb{R}} \subset G L(E)$. Пусть $M_{0} \subset E-E_{0}$ и $p_{0} \in \overline{M_{0}} \cap E_{0}$. Тогда существуют непостоянное полиномиальное отображение $\psi: \mathbb{R} \rightarrow E_{0}$ и последовательности $m_{i} \in M_{0}, t_{i} \in \mathbb{R}$ такие, что $\psi(0)=p_{0}$ и $m_{i} V_{s t_{i}} \rightarrow \psi(s), i \rightarrow \infty$, для любого $s \in \mathbb{R}$. 
Нетрудно видеть, что орбита $\sigma_{0} \circ G$ замкнута в $E$ и поэтому гомеоморфина однородному пространству $H \backslash G$. Кроме того, действие $V$ на $E$ унипотентно и подпространство $E_{0}=\mathbb{R} \sigma_{0}+\mathbb{R} q_{0} \subset E$ состоит из всех $V$-неподвижных точек. Непосредственная проверка показывает, что $E_{0} \cap G=\sigma_{0}+\mathbb{R} q_{0}=\sigma_{0} \circ U$. Пусть $M_{0}=\sigma_{0} \circ M^{-1}$. Нетрудно проверить, что $M_{0} \cap E_{0}=\varnothing$, так как $M \subset G-U H$.

Предложение 14.22 , примененное к точке $\sigma_{0} \in \overline{M_{0}} \cap E_{0}$, снабжает нас полиномиальным отображением $\psi: \mathbb{R} \rightarrow E_{0}$ и последовательностями $g_{i} \in M, t_{i} \in \mathbb{R}$ такими, что $\psi(0)=\sigma_{0}$ и $\sigma_{0} \circ\left(g_{i}^{-1} V_{s t_{i}}\right) \rightarrow \psi(s), s \in \mathbb{R}$. Так как $\psi(\mathbb{R}) \subset E_{0} \cap G$, найдется полином $\varphi: \mathbb{R} \rightarrow \mathbb{R}$ такой, что $\psi(s)=\sigma_{0} \circ U_{\varphi(s)}, s \in \mathbb{R}$. Таким образом, $H g_{i}^{-1} V_{s t_{i}} \rightarrow H U_{\varphi(s)}$ и, следовательно, $\overline{V M H} \supset U_{\varphi(\mathbb{R})}$. Но так как $\varphi(0)=0$ и $\varphi \neq$ const, множество $\varphi(\mathbb{R})$ содержит либо $\mathbb{R}^{+}$, либо $\mathbb{R}^{-}$, и лемма 14.17 , а вместе с ней и теорема 14.12 доказаны.

\section{$\S$ 15. Некоторые нерешенные проблемы}

Мы упомянем несколько проблем, так или иначе связанных с рассмотренной тематикой (за исключением уже упомянутых в $\S \S 10-12)$.

1. Теорема жесткости Ратнер утверждает, что метрический изоморфизм орициклических потоков имеет алгебраическое происхождение. Этот результат был распространен Витте до теоремы о метрической жесткости эргодических унипотентных потоков. С другой стороны, как упоминалось в $\S 2$, топологическая эквивалентность орициклических потоков на компактных поверхностях постоянной отрицательной кривизны согласно Маркусу также влечет их аффинную эквивалентность. Как следует из результатов Бенардете [Ben], топологическая эквивалентность двух эргодических унипотентных потоков на $\Gamma \backslash G$ и $\Gamma^{\prime} \backslash G^{\prime}$ влечет аффинную эквивалентность (с точностью до линейной замены времени) при условии, что изоморфизм фундаментальных групп (индуцированньй топологической эквивалентностью) продолжается до аффинного изоморфизма однородных пространств. Это условие выполнено, если группа $G$ (а тогда автоматически и $G^{\prime}$ ) нильпотентна (теорема жесткости Мальцева $[\mathrm{Rag}])$. Также согласно теореме жесткости Мостова-Маргулиса-Прасада (см. [Ma90а]) оно вьполнено, если $G$ - полупростая группа без центра, компактных множителей и нормальной подгруппы $H \subset G$ такой, что $H \simeq P S L(2, \mathbb{R})$ и произведение $Г H$ замкнуто. Однако, как показывает результат Маркуса, топологическая жесткость имеет место и для унипотентных потоков на компактных однородных пространствах вида $\Gamma \backslash P S L(2, \mathbb{R}$ ) (причем, возможно, компактность здесь несущественна). Создается впечатление, что топологическая жесткость эргодических унипотентных потоков должна иметь место в общем случае.

2. Как доказала Ратнер [R78], орициклический поток $u_{t}$ на пространстве $\Gamma \backslash S L(2, \mathbb{R})$ конечного объема эквивалентен по Какутани прямолинейному эргодическому потоку на торе. А именно, найдется такая положительная функция $\tau \in L^{1}(\Gamma \backslash S L(2, \mathbb{R}), \nu)$, что если $v(x, t)$ является решением уравнения $\int_{0}^{v(x, t)} \tau\left(x u_{s}\right) d s=t$, то поток $u_{t}^{\tau}$ на $\Gamma \backslash S L(2, \mathbb{R})$, действующий по правилу $u_{t}^{\tau}(x)=x u_{v(x, t)}$, относительно инвариантной меры $\tau \nu$ метрически изоморфен прямолинейному потоку на торе. Однако диагональньй поток $u_{t} \times u_{t}$ на $\Gamma \backslash S L(2, \mathbb{R}) \times \Gamma \backslash S L(2, \mathbb{R})$ таким свойством не обладает [R79]. В [R95b] поставлена задача найти все унипотентные потоки, эквивалентные по Какутани прямолинейно- 
му потоку. Там же задан вопрос: верно ли, что эргодические унипотентные потоки на одном и том же однородном пространстве эквивалентны по Какутани?

С другой стороны, даже слабая гладкость замены времени сохраняет свойство жесткости: метрический изоморфизм $\left(\Gamma_{1} \backslash S L(2, \mathbb{R}), u_{t}^{\tau_{1}}\right)$ и $\left(\Gamma_{2} \backslash S L(2, \mathbb{R}), u_{t}^{\tau_{2}}\right)$ влечет сопряженность $\Gamma_{1}$ и $\Gamma_{2}$ в $S L(2, \mathbb{R})$ (см. $\left.[\mathrm{R} 86]\right)$. Это так, если обе функции $\tau_{i}$ ограничены вместе с $1 / \tau_{i}$, измеримы и гёльдеровские в направлении подгруппы врашений $S O(2, \mathbb{R})=\{r(t), t \in[-\pi, \pi]\} \subset S L(2, \mathbb{R})$, т.е. найдутся константы $C, \alpha>0$ такие, что $\left|\tau_{i}(x)-\tau_{i}(x r(t))\right|<C|t|^{\alpha}$ для всех $x \in \Gamma_{i} \backslash S L(2, \mathbb{R}), t \in[-\pi, \pi]$. Возникает вопpoc (cм. [R95b]): верно ли, что метрический изоморфизм двух потоков, полученных с помошњю гладкой замены времени из эргодических унипотентных потоков, влечет афффинную эквивалентность исходных потоков?

3. Свойство перемешивания для однопараметрического потока $\left(\Gamma \backslash G, g_{\mathbb{R}}\right)$ означает, что для функций $\varphi, \psi \in L^{2}(\Gamma \backslash G, \nu)$ с нулевым средним их коэффициент корреляции $c_{t}=\int_{\Gamma \backslash G} \varphi\left(x g_{t}\right) \psi(x) d \nu_{x}$ стремится к нулю при $t \rightarrow \infty$. Часто весьма важной является информация о скорости убьвания коррелящий, и первые результаты в этом направлении с помощью теории представлений были получены еще Хариш-Чандрой. Скажем, что скорость убьвания коррелящий экспоненциальная, если $\left|c_{t}\right| \leqslant C e^{-\alpha t}$, где $\alpha=\alpha\left(G, \Gamma, g_{\mathbb{R}}\right)>0$ и $C=C(\varphi, \psi)$. Ратнер [R87] доказала экспоненциальность убывания корреляций для геодезического потока при условии, что функции $\varphi, \psi$ гёльдеровские в направлении подгруппы вращений (аналогичные результаты были также получены Муром). Для полупростой групшы $G$ без простых факторов, локально изоморфных $S O(n, 1)$ или $S U(n, 1)$, экспоненшиальность убывания коррелящий относительно $\mathbb{R}$-диагонализуемой подгрупшы $g_{\mathbb{R}} \subset G$ для $C^{\infty}$-гладких функций недавно доказана Катком и Спатциером [KS94]. В работе Клейнбока и Маргулиса [KM96] этот результат был распространен на гёльдеровские функции $\varphi, \psi$. Неизвестно, имеет ли место экспоненщиальность убывания коррелящий относительно $\mathbb{R}$-диагонализуемой подгруппы для произвольной полупростой группы $G$. Если это так, то скорость убывания корреляций для унипотентного потока - полиномиальная (как указано в [KM96], это вьводится из разложения $G=K A K$, где $K$ - максимальная компактная подгруппа и $A$ - максимальная $\mathbb{R}$-диагонализуемая подгруппа в $G$ ). То, что для орициклического потока скорость убьвания корреляций полиномиальная, было доказано Ратнер [R87].

\section{СПИСОК ЛИТЕРАТУРЫ}

[An] Аносов Д. В. Геодезические потоки на замкнутых римановых многообразиях отрицательной кривизны // Труды МИАН. 1967. Т. 90. № 5. С. 1-210.

[AG] Auslander L., Green L. G-induced flows // Amer. J. Math. 1966. V. 88. P. 43-60.

[AGH] Ауслендер Л., Грин Л., Хан Ф. Потоки на однородных пространствах. М.: Мир, 1966 .

[AS] Аносов Д. В., Солодов В. В. Гиперболические множества // Итоги науки и техники. Совр. пробл. матем. Фунд. напр. Т. 66. М.: ВИНИТИ, 1991. С. 12-99.

[Au Auslander L. An exposition of the structure of solvmanifolds // Bull. Amer. Math. Soc. 1973. V. 79. P. 227-261.

[B] Borel A. Values of indefinite quadratic forms at integral points and flows on spaces of lattices // Bull. Amer. Math. Soc. 1995. V. 32. № 2. P. 184-204. 
[BB] Bien F., Borel A. Sous-groupes épimorphiques de groupes algébriques linéaires, I // C. R. Acad. Sci. Paris. 1992. V. 315. P. 649-655.

[Be] Berend D. Minimal sets on tori // Ergodic Theory Dynam. Systems. 1984. V. 4. P. 499-507.

[Ben] Benardete D. Topological equivalence of flows on homogeneous spaces, and divergence of one-parameter subgroups of Lie groups // Trans. Amer. Math. Soc. 1988. V. 306. № 2. P. 499-527.

[BM] Brezin J., Moore C.C. Flows on homogeneous spaces: a new look // Amer. J. Math. 1981. V. 103. № 3. P. 571-613.

[Bo71] Bowen R. Entropy for group endomorphisms and homogeneous spaces // Trans. Amer. Math. Soc. 1971. V. 153. P. 401-414.

[Bo75] Bowen R. Equilibrium States and the Ergodic Theory of Anosov Diffeomorphisms. Berlin: Springer-Verlag, 1975.

[Bo76] Bowen R. Weak mixing and unique ergodicity on homogeneous spaces // Israel J. Math. 1976. V. 23. № 3. P. 267-273.

[BP] Borel A., Prasad G. Values of isotropic quadratic forms at S-integral points // Compositio Math. 1992. V. 83. P. 347-372.

[BS] Burger M., Sarnak P. Ramanujan duals II // Invent. Math. 1991. V. 106. P. 1-11.

[Bu] Burger M. Horocycle flow on geometrically finite surfaces // Duke Math. J. 1990. V. 61. P. 779-803.

[Ca57] Cassels J. W.S. An Introduction to Diophantine Approximation. Cambridge: Cambridge Univ. Press, 1957.

[Ca78] Cassels J. W.S. Rational Quadratic Forms. London: Academic Press, 1978.

[Cr] Croke C. Rigidity for surfaces of non-positive curvature // Comment. Math. Helv. 1990. V. 65. № 1. P. 150-169.

[CS] Cassels J.W.S., Swinnerton-Dyer H.P.F. On the product of three homogeneous forms and indefinite ternary quadratic forms // Philos. Trans. Roy. Soc. London. 1955. V. 248. P. 73-96.

[Da76] Dani S.G. Kolmogorov automorphisms on homogeneous spaces // Amer. J. Math. 1976. V. 98. № 1. P. 119-163.

[Da77] Dani S. G. Spectrum of an affine transformations // Duke Math. J. 1977. V. 44. P. $125-156$.

[Da80] Dani S. G. A simple proof of Borel's density theorem // Math. Z. 1980. V. 174. P. 81-94.

[Da81] Dani S.G. Invariant measures and minimal sets of horospherical flows // Invent. Math. 1981. V. 64. № 2. P. 357-385.

[Da82] Dani S.G. On uniformly distributed orbits of certain horocycle flows // Ergodic Theory Dynam. Systems. 1982. V. 2. № 2. P. 139-158.

[Da84] Dani S.G. On orbits of unipotent flows on homogeneous spaces // Ergodic Theory Dynam. Systems. 1984. V. 4. P. 25-34.

[Da86a] Dani S.G. Orbits of horospherical flows // Duke Math. J. 1986. V. 53. № 1. P. 177-188.

[Da86b] Dani S. G. On orbits of unipotent flows on homogeneous spaces, II // Ergodic Theory Dynam. Systems. 1986. V. 6. P. 167-182.

[Da86c] Dani S. G. Bounded orbits of flows on homogeneous spaces // Comment. Math. Helv. 1986. V. 61. P. 636-660.

[Da89] Dani S.G. Dense orbits of horospherical flows // Banach Center Publ. 1989. V. 23. P. 173-195.

[Da95] Dani S. G. Flows on homogeneous spaces and Diophantine approximation // Proc. of the ICM-94 (Zurich, Switzerland, 1995). P. 780-789.

[DM89] Dani S. G., Margulis G. A. Values of quadratic forms at primitive integral points // Invent. Math. 1989. V. 98. P. 405-424. 
[DM90a] Dani S. G., Margulis G. A. Orbit closures of generic unipotent flows on homogeneous spaces of $S L(3, R) / /$ Math. Ann. 1990. V. 286. P. 101-128.

[DM90b] Dani S. G., Margulis G. A. Values of quadratic forms at integral points: an elementary approach // Enseign. Math. 1990. V. 36. P. 143-174.

[DM93] Dani S.G., Margulis G.A. Limit distributions of orbits of unipotent flows and values of quadratic forms // Adv. Soviet Math. 1993. V. 16. P. 91-137.

[DR] Davenport H., Ridout H. Undefinite quadratic forms // Proc. London Math. Soc. 1959. V. 9. P. 544-555.

[DRS] Duke W., Rudnick Z., Sarnak P. Density of integer points on affine homogeneous varieties // Duke Math. J. 1993. V. 71. P. 143-180.

[DS] Dani S.G., Smillie J. Uniform distribution of horocycle flows for Fuchsian groups // Duke Math. J. 1984. V. 51. P. 185-194.

[DSMS] Dixon J. D., du Sautou M. P. F., Mann A., Segal D. Analytic Pro-p Groups. Cambridge: Cambridge Univ. Press, 1991.

[EM] Eskin A., McMullen C. Mixing, counting and equidistribution in Lie groups // Duke Math. J. 1993. V. 71. P. 181-209.

[EMM] Eskin A., Margulis G. A., Mozes S. Upper Bounds and Asymptotics in a Quantitative Version of the Oppenheim Conjecture // Preprint.

[EMS96] Eskin A., Mozes S., Shah N. Unipotent flows and counting lattice points on homogeneous varieties // Ann. Math. 1996. V. 143. P. 253-299.

[EMS97] Eskin A., Mozes S., Shah N. Non-divergence of translates of certain algebraic measures // Geom. Funct. Anal. 1997. V. 7. P. 48-80.

[EP] Ellis R., Perrizo W. Unique ergodicity of flows on homogeneous spaces // Israel J. Math. 1978. V. 29. № 2. P. 276-284.

[F] Flaminio L. An extension of Ratner's rigidity theorem to $n$-dimensional hyperbolic space // Ergodic Theory Dynam. Systems. 1987. V. 7. P. 73-92.

[FO] Feldman J., Ornstein D. Semi-rigidity of horocycle flows over compact surfaces of variable negative curvature // Ergodic Theory Dynam. Systems. 1987. V. 7. № 1. P. $49-72$.

[FS] Flaminio L., Spatzier R. Geometrically finite groups, Patterson-Sullivan measures and Ratner's rigidity theorem // Invent. Math. 1990. V. 99. №3. P. 601-626.

[Fu61] Furstenberg H. Strict ergodicity and transformations of the torus // Amer. J. Math. 1961. V. 83. P. 573-601.

[Fu72] Furstenberg H. The unique ergodicity of the horocycle flow // Recent Advances in Topological Dynamics / ed. A. Besk. Berlin: Springer-Verlag, 1972. P. 95-115.

[GF] Гельфанд И. М., Фомин С. В. Геодезические потоки на многообразиях постоянной отрицательной кривизны // УМН. 1952. Т. 7. № 1. С. 118-137.

[Gh] Ghys E. Dynamique des flots unipotents sur les espaces homogènes // Astérisque. 1992. V. 206. P. 93-136.

[Gu] Гуревич Б. М. Энтропия потока орициклов // Докл. АН СССР. 1961. Т. 136. № 4. C. $768-770$.

[GW] Glasner S., Weiss B. Minimal transformations with no common factors need not be disjoint // Israel J. Math. 1983. V. 45. № 1. P. 1-8.

[H] Herman M. Construction of a minimal diffeomorphism with nonzero topological entropy // Ergodic Theory Dynam. Systems. 1981. V. 1. P. 65-76.

[He36] Hedlund G. A. Fuchsian groups and transitive horocycles // Duke Math. J. 1936. V. 2. P. 530-542.

[He39] Hedlund G.A. The dynamics of geodesic flows // Bull. Amer. Math. Soc. 1939. V. 45. P. 241-260.

[Ho] Hopf E. Fuchsian groups and ergodic theory // Trans. Amer. Math. Soc. 1936. V. 39. № 2. Р. 299-314.

[Нu] Хамфри Дж. Линейные алгебраические группы. М.: Наука, 1980.

[Ja] Джекобсон Н. Алгебры Ли. М.: Мир, 1964. 
[Jo] Johnson R. On almost periodic linear differential systems of Millionshchikov and Vino$\operatorname{grad}$ // J. Math. Anal. Appl. 1982. V. 85. № 2. P. 452-461.

[K75] Каток А. Б. Замена времени, монотонная эквивалентность и стандартные динамические системы // Докл. АН СССР. 1975. V. 223. № 4. Р. 789-792.

[K82] Katok A. B. Entropy and closed geodesics // Ergodic Theory Dynam. Systems. 1982. V. 2. № 3-4. P. 339-365.

[Ka] Kalikow S. A. Twofold mixing implies threefold mixing for rank one transformations // Ergodic Theory Dynam. Systems. 1984. V. 4. P. 237-259.

[KM96] Kleinbock D.Y., Margulis G. A. Bounded orbits of non-quasiunipotent flows on homogeneous spaces // Amer. Math. Soc. Transl. 1996. V. 171. P. 141-171.

[KM97] Kleinbock D. Y., Margulis G. A. Flows on Homogeneous Spaces and Diophantine Approximation on Manifolds // Preprint.

[KS94] Katok A., Spatzier R. J. First cohomology of Anosov actions of higher rank abelian groups and applications to rigidity // IHES Publ. Math. 1994. V. 79. P. 131-156.

[KS96] Katok A., Spatzier R.J. Invariant measures for higher rank hyperbolic abelian actions // Ergodic Theory Dynam. Systems. 1996. V. 16. P. 751-778.

[KSF] Корнфельд И. П., Синай Я.Г., Фомин С. В. Эргодическая теория. М.: Наука, 1980 .

[Ku] Кушниренко А. Г. Оценка сверху энтропии классических динамических систем // Докл. АН СССР. 1965. Т. 161. №1. С. 37-38.

[KW] Katznelson Y., Weiss B. When all points are recurrent/generic. // Ergodic Theory and Dynamical Systems / ed. A. Katok. V. I (Proceedings, Special year, Maryland, 1979-80). Boston: Birkhäuser, 1981.

[L] Ledrappier F. Un champ markovien peut etre d'entropie nulle et mélangeant // C. R. Acad. Sci. Paris. 1978. V. 287. P. 561-563.

[Le] Lesigne E. Théorèmes ergodiques pour une translation sur nilvariété // Ergodic Theory Dynam. Systems. 1989. V. 9. P. 115-126.

[LY] Ledrappier F., Young L.-S. The metric theory of diffeomorphisms // Ann. Math. 1985. V. 122. P. 503-539.

[M] Мальцев А. И. О разрешимых алгебрах Ли // Изв. АН СССР. Сер. матем. 1945. T. 9. C. $329-352$.

[Ma70] Маргулис Г. А. О некоторых мерах, связанных с У-потоками на компактных многообразиях // Функц. анализ и его прил. 1970. Т. 4. №1. С. 62-76.

[Ma71] Маргулис Г.А. О действии унипотентных подгрупп в пространстве решеток // Матем. сб. 1971. Т. 86. № 4. С. 552-556.

[Ma87] Margulis G. A. Formes quadratiques indefinies et flots unipotents sur les espaces homogénes // C. R. Acad. Sci. Paris. 1987. V. 304. № 10. P. 249-253.

[Ma88] Margulis G. A. Lie groups and ergodic theory // Proc. of Conference in Varna (1986). Berlin: Springer-Verlag, 1988. P. 130-146.

[Ma89] Margulis G. A. Discrete subgroups and ergodic theory // Proc. of the conference in honour of A. Selberg (Oslo, 1987). New York: Academic Press, 1989. P. 377-398.

[Ma90a] Margulis G. A. Discrete subgroups of semisimple groups. Berlin: Springer-Verlag, 1990.

[Ma90b] Margulis G. A. Orbits of group actions and values of quadratic forms at integral points // Israel Mathematical Conference Proceedings "Festschrift in honour of I. I. Piatetski-Shapiro". The Weizmann Science Press of Israel. V. 3, 1990. P. 127-151.

[Ma91a] Margulis G. A. Compactness of minimal closed invariant sets of actions of unipotent groups // Geom. Dedicata. 1991. V. 37. P. 1-7.

[Ma91b] Margulis G. A. Dynamical and ergodic properties of subgroups actions on homogeneous spaces with applications to number theory // ICM-90 (Math. Soc. Japan, Kyoto), 1991. P. 193-215.

[Ma96] Margulis G. A. Oppenheim Conjecture // Preprint. 
[Mar75] Marcus B. Unique ergodicity of the horocycle flow: the variable curvature case // Israel J. Math. 1975. V. 21. P. 131-144.

[Mar78] Marcus B. The horocycle flow is mixing of all degrees // Invent. Math. 1978. V. 46. P. 201-209.

[Mar83] Marcus B. Topological conjugacy of horocycle flows // Amer. J. Math. 1983. V. 105. P. $623-632$.

[Mi68] Миллионщиков В. М. Доказательство существования неправильных систем линейных дифференциальных уравнений с почти периодическими коэффициентами // Дифференц. уравнения. 1968. Т. 4. №3. С. 391-396.

[Mi76] Миллионщиков В. М. Формула для энтропии гладкой динамической системы // Дифференц. уравнения. 1976. Т. 12. №12. С. 2188-2192.

[Mo66] Moore C.C. Ergodicity of flows on homogeneous spaces // Amer. J. Math. 1966. V. 88. P. $154-178$.

[Mo80] Moore C. C. The Mautner phenomenon for general unitary representations // Pacific J. Math. 1980. V. 86. №1. P. 154-169.

[Mor] Morse H. M. Recurrent geodesics on surface of negative curvature // Trans. Amer. Math. Soc. 1921. V. 22. №1. P. 84-100.

[Moz92] Mozes S. Mixing of all orders of Lie group actions // Invent. Math. 1992. V. 107. P. 235-241.

[Moz95a] Mozes S. Epimorphic subgroups and invariant measures // Ergodic Theory Dynam. Systems. 1995. V. 15. P. 1207-1210.

[Moz95b] Mozes S. Correction to the paper "Mixing of all orders of Lie group actions" // Invent. Math. 1995. V. 119. P. 399.

[MS] Mozes S., Shah N. On the space of ergodic invariant measures of unipotent flows // Ergodic Theory Dynam. Systems. 1995. V. 15. № 1. P. 149-159.

[MT94] Margulis G. A., Tomanov G. Invariant measures for actions of unipotent groups over local fields on homogeneous spaces // Invent. Math. 1994. V. 116. P. 347-392.

[MT96] Margulis G. A., Tomanov G. Measure rigidity for almost linear groups and its applications // J. Anal. Math. 1996. V. 69. P. 25-54.

[Ne] Немыцкий В. В. Топологические вопросы теории динамических систем // УМН. 1949. Т. 4. №6. С. 91-153.

[O] Орнстейн Д. Эргодическая теория, случайность и динамические системы. М.: Мир, 1978.

[Op] Oppengeim A. Values of quadratic forms I, II // Quart. J. Math. Oxford. 1953. V. 4. P. 54-59, 60-66.

[Ot] Otal J.-P. Le spectre marqué des longueurs des surfaces à courbure négative // Ann. Math. 1990. V. 131. №1. P. 151-162.

[OW] Ornstein D., Weiss B. Geodesic flows are Bernoullian // Israel J. Math. 1973. V. 14. P. 184-198.

[P69] Parry W. Ergodic properties of affine transformations and flows on nilmanifolds // Amer. J. Math. 1969. V. 91. № 3. P. 751-777.

[P71] Parry W. Metric classification of ergodic nilflows and unipotent affines // Amer. J. Math. 1971. V. 93. P. 819-828.

[P73] Parry W. Dynamical representation in nilmanifolds // Compos. Math. 1973. V. 26. P. $150-174$

[Pa] Парасюк О.С. Поток гороциклов на поверхностях постоянной отрицательной кривизны // УМН. 1953. Т. 8. № 3. С. 124-126.

[Ре] Песин Я. Б. Характеристические показатели Ляпунова и гладкая эргодическая теория // УМН. 1977. Т. 32. № 4. С. 55-111.

[Pi] Пинскер М. С. Динамические системы с вполне положительной и нулевой энтропией // Докл. АН СССР. 1960. Т. 133. № 5. С. 1025-1026.

[PS] Pugh C., Shub M. Ergodic elements of ergodic actions // Compositio Math. 1971. V. 23. P. $115-122$. 
[R78] Ratner M. Horocycle flows are loosely Bernoullian // Israel J. Math. 1978. V. 31. № 2. P. 122-132.

[R79] Ratner M. The Cartesian square of the horocycle flow is not loosely Bernoullian // Israel J. Math. 1978. V. 34. № 1-2. P. 72-96.

[R82a] Ratner M. Rigidity of horocycle flows // Ann. Math. 1982. V. 115. P. 597-614.

[R82b] Ratner M. Factors of horocycle flows // Ergodic Theory Dynam. Systems. 1982. V. 2. P. $465-489$.

[R83] Ratner M. Horocycle flows, joinings and rigidity of products // Ann. Math. 1983. V. 118. P. 277-313.

[R84] Ratner M. Ergodic theory in hyperbolic space // Contemp. Math. 1984. V. 26. P. 309-334.

[R86] Ratner M. Rigidity of time changes for horocycle fows // Acta Math. 1986. V. 156. P. 1-32.

[R87] Ratner M. The rate of mixing for geodesic and horocycle flows // Ergodic Theory Dynam. Systems. 1987. V. 7. P. 267-288.

[R90a] Ratner M. Invariant measures for unipotent translations on homogeneous spaces // Proc. Nat. Acad. Sci. USA. 1990. V. 87. P. 4309-4311.

[R90b] Ratner M. Strict measure rigidity for unipotent subgroups of solvable groups // Invent. Math. 1990. V. 101. P. 449-482.

[R90c] Ratner M. On measure rigidity of unipotent subgroups of semisimple groups // Acta Math. 1990. V. 165. P. 229-309.

[R91a] Ratner M. On Raghunathan's measure conjecture // Ann. Math. 1991. V. 134. P. 545-607.

[R91b] Ratner M. Raghunathan's topological conjecture and distributions of unipotent flows // Duke Math. J. 1991. V. 63. P. 235-280.

[R92] Ratner M. Raghunathan's conjecture for $S L(2, \mathbb{R})$ // Israel J. Math. 1992. V. 80. P. 1-31.

[R93] Ratner M. Raghunathan's conjectures for $p$-adic Lie groups // Internat. Math. Res. Notices. 1993. № 5. P. 141-146.

[R94] Ratner M. Invariant measures and orbit closures for unipotent actions on homogeneous spaces // Geom. Funct. Anal. 1994. V. 4. P. 236-256.

[R95a] Ratner M. Raghunathan's conjectures for cartesian products of real and $p$-adic Lie groups // Duke Math. J. 1995. V. 77. № 2. P. 275-382.

[R95b] Ratner M. Interactions between ergodic theory, Lie groups, and number theory // ICM-94 (Zurich, Switzerland), 1995. P. 157-182.

[Rag] Рагунатан М. Дискретные подгруппы групп Ли. М.: Мир, 1977.

[Re] Remage R. On minimal sets in the plane // Proc. Amer. Math. Soc. 1962. V. 13. P. 41-47.

[Ry91] Рыжиков B. В. Связь перемешивающих свойств потока с изоморфизмами входящих в него преобразований // Матем. заметки. 1991. Т. 49. №6. С. 98-106.

[Ry93] Рыжиков В. В. Джойнинги и кратное перемешивание для действий конечного ранга // Функц. анализ и его прил. 1993. Т. 27. № 2. С. 63-78.

[Ry95] Рыжиков B. В. Функциональный взгляд на теорему Семереди. Замечание о кратном перемешивании потоков // УМН. 1995. Т. 50. №6. С. 213-214.

[Sa] Sarnak P. Asymptotic behaviour of periodic orbits of the horocycle flow and Eisenstein series // Comm. Pure Appl. Math. 1981. V. 34. P. 719-739.

[Saf] Сафонов А. В. О спектралшном типе эргодических индуцированных потоков // Изв. вузов. Сер. матем. 1982. Т. 241. №6. С. 242-247.

[Sch] Schmidt W. M. On badly approximable numbers and certain games // Trans. Amer. Math. Soc. 1966. V. 123. P. 178-199.

[Se69] Сepp ЖК.-П. Алгебры Ли и группы Ли. М.: Мир, 1969.

[Se82] Серр ЖК.-П. Курс арифметики. М.: Мир, 1982. 
[Sh91] Shah N. Uniformly distributed orbits of certain flows on homogeneous spaces // Math. Ann. 1991. V. 289. P. 315-334.

[Sh94] Shah N. Limit distributions of polynomial trajectories on homogeneous spaces // Duke Math. J. 1994. V. 75. № 3. P. 711-732.

[Sh96] Shah N. Limit distributions of expanding translates of certain orbits on homogeneous spaces // Proc. Indian Acad. Sci. Math. Sci. 1996. V. 106. № 2. P. 105-125.

[Sh97] Shah N. Invariant measures and orbit closures on homogeneous spaces for actions of subgroups generated by unipotent elements // Conference on Lie Groups and Ergodic Theory Proceedings, TIFR, Bombay, 1996 (to appear).

[Si60] Синай Я. Г. Геодезические потоки на многообразиях постоянной кривизны // Докл. AH CCCP. 1960. T. 131. № 4. C. 752-755.

[Si72] Синай Я. Г. Гиббсовские меры в эргодической теории // УМН. 1972. Т. 27. № 4. C. 21-63.

[Skr] Skriganov M.M. Ergodic theory on $S L(n)$, Diophantine approximations and anomalies in the lattice point problem // Steklov Math. Inst., St. Petersb. Dept., Preprint, 1996.

[Sp] Спринджук В.Г. Достижения и проблемы диофантовых приближений // УМН. 1980. Т. 35. № 4. С. 3-68.

[SSS] Сафонов А. В., Старков А.Н., Степин А. М. Динамические системы с транзитивной группой симметрий // Итоги науки и техники. Совр. пробл. матем. Фунд. напр. Т. 66. М.: ВИНИТИ, 1991. С. 187-243.

[St83] Старков А.Н. Эргодическое поведение потоков на однородных пространствах // Докл. АН СССР. 1983. Т. 273. № 3. С. 538-540.

[St84] Старков А.Н. Потоки на компактных разрешимых многообразиях // Матем. сб. 1984. T. 123. № 4. C. 549-556.

[St86] Старков А.Н. О пространствах конечного объема // Вестник МГУ. Сер. матем. 1986. № 5. C. 64-66.

[St87a] Старков А.Н. О критерии эргодичности $G$-индуцированных потоков // УМН. 1987. Т. 42. № 3. С. 197-198.

[St87b] Старков А.Н. Разрешимые однородные потоки // Матем. сб. 1987. Т. 134. № 2. C. $242-259$.

[St88] Старков А.Н. Редукция теории однородных потоков к случаю дискретной подгруппы изотропии // Докл. АН СССР. 1988. Т. 301. № 6. С. 1328-1331.

[St89] Старков А.Н. Эргодическое разложение потоков на однородных пространствах конечного объема // Матем. сб. 1989. Т. 180. № 12. С. 1614-1633.

[St90] Старков А.Н. Структура орбит однородных потоков и гипотеза Рагунатана // УМH. 1990. T. 45. C. 219-220.

[St91] Старков А.Н. Орисферические потоки на пространствах конечного объема // Матем. сб. 1991. Т. 182. № 5. С. 774-784.

[St93] Старков А.Н. О кратном перемешивании однородных потоков // Докл. АН. 1993. T. 333. № 4. C. 28-31.

[St95a] Starkov A. N. Minimal sets of homogeneous flows // Ergodic Theory Dynam. Systems. 1995. V. 15. P. 361-377.

[St95b] Starkov A. N. Fuchsian groups from the dynamical viewpoint // J. Dynam. Contr. Syst. 1995. V. 3. P. 427-445.

[St95c] Старков А.Н. О взаимных изоморфизмах трансляций однородного потока // Матем. заметки. 1995. Т. 58. №1. С. 98-110.

[Ste69] Степин А. М. Потоки на разрешимых многообразиях // УМН. 1969. Т. 24. № 2. C. 241-242.

[Ste73] Степин A.M. Динамические системы на однородных пространствах полупростых групп Ли // Изв. АН СССР. Сер. матем. 1973. Т. 37. № 5. С. 1091-1107.

[T] Tamagawa T. Arithmetic Algebraic Geometry. New York: Harper and Row, 1965. 
[Th] Thouvenot J.-P. Some Properties and Applications of Joinings in Ergodic Theory // London Math. Soc. Lecture Notes Ser. V. 205, 1995.

[V] Veech W. A. Unique ergodicity of horospherical flows // Amer. J. Math. 1977. V. 99. P. 827-859.

[VdV] Ван дер Варден Б. Л. Алгебра. М.: Наука, 1979.

[Ve] Verjovsky A. Arithmetic, geometry and dynamics in the unit tangent bundle of the modular orbifold // Preprint ICTP.

[VGS] Винберг Э. Б., Горбацевич В. В., Шварцман О.В.Дискретные подгруппы групп Ли // Итоги науки и техники. Совр. пробл. матем. Фунд. напр. Т. 21. М.: ВИНИТИ, 1985. С. 5-120.

[Vi] Виноград Р.Э. К проблеме Н.П. Еругина // Дифференц. уравнения. 1975. V. 11. Р. 632-638.

[VO] Винберг Э. Б., Онищик А. Л. Семинар по группам Ли и алгебраическим группам. М.: Наука, 1988.

[VS] Винберг Э. Б., Шварцман О.В.Дискретные группы движения пространств постоянной кривизны // Итоги науки и техники. Совр. пробл. матем. Фунд. напр. Т. 29. М.: ВИНИТИ, 1988. С. 147-259.

[Wi85] Witte D. Rigidity of some translations on homogeneous spaces // Invent. Math. 1985. V. 81. P. 1-27.

[Wi87] Witte D. Zero-entropy affine maps on homogeneous spaces // Amer. J. Math. 1987. V. 109. P. 927-961.

[Wi89] Witte D. Rigidity of horospherical foliations // Ergodic Theory Dynam. Systems. 1989. V. 9. P. 191-205.

[Wi94] Witte D. Measurable quotients of unipotent translations on homogeneous spaces // Trans. Amer. Math. Soc. 1994. V. 345. № 2. P. 577-594.

[Wi96] Witte D. Correction to the paper "Zero-entropy affine maps on homogeneous spaces" // Amer. J. Math. (to appear).

[Z] Zimmer R. Ergodic theory and semisimple groups. Boston: Birkhäuser, 1984.

[Za] Zagier D. Eisenstein series and the Riemann zeta-function // Automorphic forms, Representation Theory and Arithmetic, TIFR, Bombay, 1981. P. 275-301.

Всероссийский электротехнический институт, г. Истра

E-mail: alex@rdiees.msk.ru
Поступила в редакцию 28.05 .1996 